Parameterized Verification of Synchronized Concurrent Programs

Zeinab Ganjei 



\title{
Parameterized Verification of Synchronized Concurrent Programs
}

\author{
Zeinab Ganjei
}

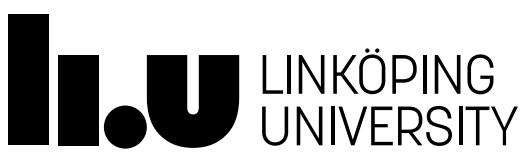

Linköping University

Department of Computer and Information Science

Division of Software and Systems

SE-581 83 Linköping, Sweden

Linköping 2021 
$(\mathrm{cc})$ EY-No NonCommercial 4.0 International License.

https://creativecommons.org/licenses/by-nc/4.0/

Edition 1:1

(c) Zeinab Ganjei, 2021

ISBN 978-91-7929-697-1

ISSN 0345-7524

URL http://urn.kb.se/resolve?urn=urn:nbn:se:liu:diva-173568

Published articles have been reprinted with permission from the respective copyright holder.

Typeset using $\mathrm{X}_{\mathrm{H}} \mathrm{T}_{\mathrm{E}} \mathrm{X}$

Printed by LiU-Tryck, Linköping 2021 


\begin{abstract}
There is currently an increasing demand for concurrent programs. Checking the correctness of concurrent programs is a complex task due to the interleavings of processes. Sometimes, violation of the correctness properties in such systems causes human or resource losses; therefore, it is crucial to check the correctness of such systems. Two main approaches to software analysis are testing and formal verification. Testing can help discover many bugs at a low cost. However, it cannot prove the correctness of a program. Formal verification, on the other hand, is the approach for proving program correctness. Model checking is a formal verification technique that is suitable for concurrent programs. It aims to automatically establish the correctness (expressed in terms of temporal properties) of a program through an exhaustive search of the behavior of the system. Model checking was initially introduced for the purpose of verifying finite-state concurrent programs, and extending it to infinitestate systems is an active research area.
\end{abstract}

In this thesis, we focus on the formal verification of parameterized systems. That is, systems in which the number of executing processes is not bounded a priori. We provide fullyautomatic and parameterized model checking techniques for establishing the correctness of safety properties for certain classes of concurrent programs. We provide an open-source prototype tool for most of the techniques and present our experimental results on several benchmarks. We also provide undecidability results for certain reachability problems.

First, we address the problem of automatically checking safety properties for bounded as well as parameterized phaser programs. Phaser programs are concurrent programs that make use of the phaser synchronization construct found in modern languages such as Habanero Java. For the bounded case, we establish the decidability of checking violation of program assertions and the undecidability of checking deadlock-freedom. For the parameterized case, we study different formulations of the verification problem and propose a procedure that can be exact and guaranteed to terminate for some reachability problems even in the presence of unbounded phases and arbitrarily many spawned processes. Second, we propose an approach for automatic verification of parameterized concurrent programs in which shared variables are manipulated by atomic transitions to count and synchronize the spawned processes. For this purpose, we introduce counting predicates that relate counters that refer to the number of processes satisfying some given properties to the variables that are directly manipulated by the concurrent processes. We then combine existing works on the counter, predicate, and constrained monotonic abstraction and build a nested counterexample-based refinement scheme to establish correctness. Third, we introduce Lazy Constrained Monotonic Abstraction for more efficient exploration of well-structured abstractions of infinite-state non-monotonic systems. We propose several heuristics and assess the efficiency of the proposed technique by extensive experiments using our open-source prototype tool. Lastly, we propose a sound but (in general) incomplete procedure for automatic verification of safety properties for a class of fault-tolerant distributed protocols described in the Heard-Of (HO for short) model. The HO model is a popular model for describing distributed protocols. We propose a verification procedure that is guaranteed to terminate even for unbounded number of the processes that execute the distributed protocol.

The research presented in this thesis has been partially funded by the National Computer Science Graduate School (CUGS) in Sweden. 


\section{POPULÄRVETENSKAPLIG SAMMANFATTNING}

Samtidiga program består av flera processer som deltar i beräkningar. Sådana processer kan, jämfört med de sekventiella varianterna, resultera i mer effektiva och tillförlitliga program. Sensornätverk som sprids i en skog för att larma om eld, inbyggda system som möjliggör användningen av olika funktioner i en modern bil eller banksystem som delar transaktioner och beräkningsbelastning på olika processorer är några exempel på system som kan köra samtidiga program. Att kontrollera att samtidiga program är korrekta, dvs. att de beter sig som förväntat, är en komplex uppgift på grund av deras invecklade beteende och det, möjligtvis, stora antalet inblandade processer. Buggar och logiska misstag i datorprogram kan orsaka mänskliga och materiella förluster. Verkliga skräckexemplen inkluderar fall där synkroniseringsfel mellan processer som kontrollerar delar av en strålbehandlingsmaskin resulterade i strålningsöverdoser för flera patienter. Att försäkra sig att samtidiga program beter sig som förväntat är därför viktigt. Formell verifiering och testning är två huvudmetoder för analys av datorprogram. Vid testning ges en uppsättning testfall som input till programmet. För varje testfall anges också det förväntade resultatet. Sedan jämförs programmets resultat mot det förväntade resultatet. Testning är väl etablerat och kan upptäcka många buggar till en låg kostnad. Testning kan dock inte bevisa att ett program är korrekt. Formell verifiering är en samling av tekniker som syftar till att bevisa programkorrekthet. Modellkontroll (model checking) är en formell verifieringsteknik som är lämplig för samtidiga program. Korrektheten är uttryckt med hjälp av formler i temporallogik. Själva verifieringen genomförs med hjälp av en uttömmande sökning av systemets beteende. Tekniken introducerades ursprungligen för att verifiera samtidiga program med ändligt antal tillstånd. Att utöka modellkontroll till system med godtyckliga tillståndstorlek är ett aktivt forskningsområde. I denna avhandling fokuserar vi på formell verifiering av parametriserade system. Det vill säga, system där antalet processer är ändligt men inte begränsas på förhand. Vi tillhandahåller helautomatiska och parametriserade modellkontrolltekniker för att etablera eller motbevisa säkerhetsegenskaper (safety properties) för valda klasser av samtidiga program. Vi presenterar våra experimentella resultat genom flera exempel. Först beaktar vi problemet med att automatiskt kontrollera säkerhetsegenskaper för phaser-program där antalet processer kan vara avgränsade eller parametriserade. Dessa samtidiga program använder sig av phasers: en komplex synkroniseringskonstruktion som implementeras i moderna språk som Habanero Java. För det avgränsade fallet fastställer vi avgörbarhet för att kontrollera om programuttryck respekteras och oavgörbarhet av att kontrollera dödläge-frihet. För det parametriserade fallet studerar vi olika formuleringar av verifieringsproblemet och föreslår en exakt procedur som garanteras avslutas för vissa nåbarhetsproblem även med obegränsade antal phaser och godtyckligt många uppkomna processer. Vi föreslår också en metod för automatisk verifiering av parametriserade program där variabler delas och manipuleras av flera processer. Vi introducerar predikat som använder speciella variabler som hänvisar till antalet processer som uppfyller vissa givna egenskaper och vanliga variabler som direkt manipuleras av det samtidiga programmet. Detta möjliggör att resonera kring relationen mellan antalet processer med vissa egenskaper och värden i programvariablerna. Dessutom introducerar vi Lazy Constrained Monotonic Abstraction för effektivare utforskning av välstrukturerade abstraktioner av icke-monotona system. Dessa är system där instanser med flera processer inte nödvändigtvis behöver kunna göra mera än instanser med färre processer. Det gör att analysen av parametriserade varianter blir svårare. Vi föreslår flera heuristik och jämför deras effektivitet med hjälp av omfattande experiment med vår öppenkällkod prototyp. Till slut föreslår vi en sund men (i allmänhet) icke-fullständig procedur för automatisk verifiering av säkerhetsegenskaper för en klass av fel-toleranta distribuerade protokoll som beskrivs i Heard-Of-modellen. Vi föreslår en verifieringsheuristik 
som garanteras avslutas även för ett obegränsat antal processer som kör det distribuerade protokollet. 



\section{Acknowledgments}

I want to begin by thanking God for He has given me the opportunity and capability to reach this stage of my life. Then, I would like to offer my sincere gratitude to my advisors, Associate Professor Ahmed Rezine, Professor Petru Eles, and Professor Zebo Peng for giving me the opportunity to pursue a $\mathrm{PhD}$ in the Embedded Systems Lab at Linköping University, for sharing their wisdom and knowledge, and most importantly, for allowing me the room to work in my own way. I cannot thank any of my professors enough for their dedication and efforts in creating an excellent working environment. Their input, understanding, and patience have been invaluable in helping me pursue my research goals.

I am especially grateful to Ahmed for his continued help and support during the whole time I was at ESLAB. I have been very fortunate to have had Ahmed as my advisor, who brightened up my academic path and supported me through each step of the way. He provided me with the necessary guidance and also ample space to improve my independent thoughts during my $\mathrm{PhD}$. I would like to thank him for teaching me lessons of patience and passion for education that I will carry throughout my life's journey, making sure I was getting all the help I needed and never letting me down. I would also like to thank Professor Ludovic Henrio for his collaboration and contribution as a co-author.

The ESLAB and IDA members contributed immensely to my personal and professional time as a $\mathrm{PhD}$ student at LiU. My profound appreciation also goes to Amir Aminifar and Breeta SenGupta for welcoming me to ESLAB and helping me take my first steps. Specifically, my most heartfelt thanks to Mina Niknafs and Åsa Kärrman, who were both my colleagues and friends.

I would like to take this opportunity and extend my thanks to Professor Mariam Kamkar for all her encouragement and kindness, to the administration at IDA that facilitated my work, and especially to Anne Moe, Inger Norén, and Lene Rosell.

Last but not least, I would like to express my deepest gratitude to my parents, to whom I shall always be indebted, for their unconditional love, for raising me, supporting me, but never asking me how much of my $\mathrm{PhD}$ was left! To my sweet and adorable little sons who brought joy and adventure 
to our family, who motivated me to take a refreshing break from my studies and contributed to the length of my $\mathrm{PhD}$ ! To my loving, caring, and patient husband, Meysam, who has always been there for me and whose sacrifices I greatly appreciate and never forget.

Zeinab Ganjei

Linköping, 2021 


\section{Contents}

Abstract $\quad$ iii

Acknowledgments viii

$\begin{array}{ll}\text { Contents } & \text { ix }\end{array}$

List of Figures $\quad$ xi

List of Tables $\quad$ xiii

1 Introduction $\quad 1$

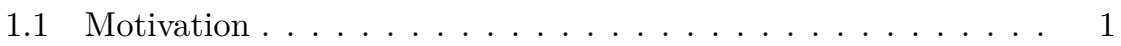

1.2 Formal Verification . . . . . . . . . . . . . . . 3

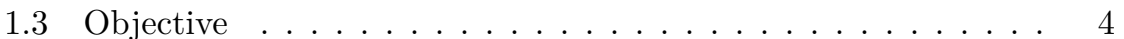

1.4 Previous Work ..................... 5

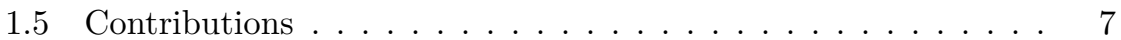

1.6 Thesis Overview .................... 8

1.7 Publication Overview .................... 9

2 Background $\quad 11$

2.1 Synchronization Mechanisms . . . . . . . . . . . . . . 11

2.2 Model Checking . . . . . . . . . . . . . . . . . . 12

2.3 Abstraction ......................... 14

$\begin{array}{llr}3 & \text { Preliminaries } & 17\end{array}$

3.1 Preorders, Configurations, and Constraints . . . . . . . . . 17

3.2 Counter Machines . . . . . . . . . . . . . . . . . . 18

3.3 Working-List Procedure . . . . . . . . . . . . . . . . 20

3.4 Monotonic Abstraction . . . . . . . . . . . . . . . . 24

4 Safety Verification of Phaser Programs 29

4.1 Introduction . . . . . . . . . . . . . . . . . . 29

4.2 Illustrative Example . . . . . . . . . . . . . . . . . . . . . . . . . . . . . . 31

4.3 Preliminaries . . . . . . . . . . . . . . . . . 34 
4.4 Phaser Programs . . . . . . . . . . . . . . . . . . 34

4.5 Symbolic Verification of Phaser Programs . . . . . . . . . . . . 44

4.6 Verification Procedure . . . . . . . . . . . . . . . . 54

4.7 Experimental Results . . . . . . . . . . . . . . . 56

4.8 Conclusion . . . . . . . . . . . . . . . 56

5 Safety Verification of Parameterized Phaser Programs $\quad 59$

5.1 Introduction . . . . . . . . . . . . . . . . . 59

5.2 Illustrative Example . . . . . . . . . . . . . . . . . . . . . . . 61

5.3 Phaser Programs and Reachability ... . . . . . . . . . . . 61

5.4 A Gap-based Symbolic Representation . . . . . . . . . . . . . . 70

5.5 A Symbolic Verification Procedure . . . . . . . . . . . . . . 79

5.6 Limitations of Deciding Reachability . . . . . . . . . . . . . . . . . 94

5.7 Conclusion . . . . . . . . . . . . . . . . . . 98

6 Counting Dynamically Synchronizing Processes 101

6.1 Introduction . . . . . . . . . . . . . . . . 101

6.2 Illustrative Example . . . . . . . . . . . . . . . . . . . . . 104

6.3 Preliminaries . . . . . . . . . . . . . . . . . 108

6.4 Concurrent Programs and Counting Predicates . . . . . . . . . 109

6.5 Relating abstraction layers . . . . . . . . . . . . . . . 113

6.6 Experimental results . . . . . . . . . . . . . . . . . . . . . . . . . . . . . . . . . . . . .

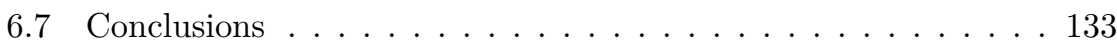

7 Lazy Constrained Monotonic Abstraction $\mathbf{1 3 5}$

7.1 Introduction . . . . . . . . . . . . . . . . . 135

7.2 Preliminaries . . . . . . . . . . . . . . . . 137

7.3 Symbolic representation . . . . . . . . . . . . . . . . . 138

7.4 Illustrative Example . . . . . . . . . . . . . . . . . . . . . . . . . . . . . . . . . . . . . . . . .

7.5 State Reachability Checking . . . . . . . . . . . . . . . . . 144

7.6 Experimental Results . . . . . . . . . . . . . . . . 151

7.7 Conclusion . . . . . . . . . . . . . . . 154

8 Verifying Safety of Parameterized Heard-Of Algorithms 155

8.1 Introduction . . . . . . . . . . . . . . . . 155

8.2 Illustrative Example . . . . . . . . . . . . . . . . . . . . . . . . . . . . . . . . . . . . . . . . . . . . . . . .

8.3 Heard-of Programs . . . . . . . . . . . . . . . . . . . . . . . 162

8.4 Symbolic Representation . . . . . . . . . . . . . . . . . . 164

8.5 A Symbolic Verification Procedure . . . . . . . . . . . . . 166

8.6 Experimental Results . . . . . . . . . . . . . . . . . 171

8.7 Conclusion and Future Work . . . . . . . . . . . . . . 172

9 Conclusion $\quad 175$

$\begin{array}{lr}\text { Bibliography } & 179\end{array}$ 


\section{List of Figures}

3.1 An overview of the CEGAR algorithm in CMA . . . . . . . . . . 26

4.1 Phaser program with two producers and one consumer . . . . . . . 32

4.2 Sample wait and signal phase values for Figure $4.1 \ldots \ldots 33$

4.3 Operational semantics of phaser statements. . . . . . . . . . . . . . 38

4.4 A sample deadlock configuration . . . . . . . . . . . . . . . . . 40

4.5 Encoding of a 3-counter reset-VAS for proof of Theorem 7 (Part I) 41

4.6 Encoding of a 3-counter reset-VAS for proof of Theorem 7 (Part II) 42

4.7 A deadlock configuration generated by proof of the Theorem 7 . . 43

4.8 Predecessor computation for phaser program statements . . . . . . 51

5.1 Parameterized producers and consumers modeled with Phasers . . 61

5.2 Operational semantics of phaser statements without errors . . . . 65

5.3 Definition of error configurations in a Phaser program . . . . . . . 66

5.4 A sample configuration generated in the proof of Theorem 12 . . 68

5.5 Encoding a 2-counter Minsky machine for the proof of Theorem 1269

5.6 Auxiliary functions used in the predecessor computation of the parameterized Phaser instructions (Part I) . . . . . . . . . 81

5.7 Auxiliary functions used in the predecessor computation of the parameterized Phaser instructions (Part II) . . . . . . . . . . . 82

5.8 Predecessor computation for parameterized Phaser statements (Part I) . . . . . . . . . . . . . . . . . . 87

5.9 Predecessor computation for parameterized Phaser statements (Part II ) . . . . . . . . . . . . . . . . . . . . . . . . . 88

5.10 Encoding a 2-counter Minsky machine for the proof of Theorem 1895

5.11 A possible configuration in proof of Theorem $18 \ldots$. . . . . . . 96

5.12 A possible configuration in proof of Theorem 18 when two tasks try to decrement the counter $x \ldots \ldots \ldots$. . . . . . . 97

5.13 Encoding a 2-counter Minsky machine for proof of Theorem 19 . . 98

6.1 A sample parameterized concurrent program in which processes synchronize using shared variables . . . . . . . . . . . . . . . 105

6.2 Predicated Constrained Monotonic Abstraction . . . . . . . . . . . 106

6.3 Syntax of concurrent programs . . . . . . . . . . . . . . . 110 
6.4 Semantics of concurrent programs . . . . . . . . . . . . . 111

6.5 Predicate abstraction of the program in Figure $6.1 \ldots \ldots$. . . . 114

6.6 Syntax of concurrent Boolean programs . . . . . . . . . . 116

6.7 Semantics of concurrent Boolean programs . . . . . . . . . . 118

6.8 Semantics of an extended counter machine . . . . . . . . . 120

6.9 Encoding of the transitions of a Boolean program given a counting target . . . . . . . . . . . . . . . . . . . 121

6.10 Strengthening of a counter machine transition . . . . . . . 127

6.11 Encoding a 2-counter Minsky machine for proof of Lemma 32 . . 130

7.1 A sample concurrent program and its encoding as a counter machine140

7.2 A spurious trace generated because of monotonic abstraction . . . 143

7.3 Comparing execution times of eager and lazy variants . . . . . . 152

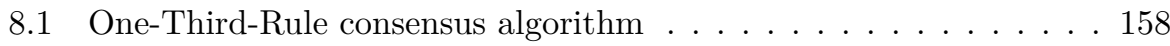

8.2 A run of One-Third-Rule algorithm . . . . . . . . . . . . . 160

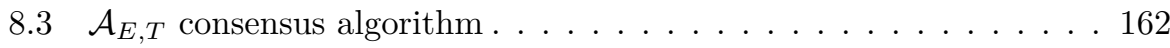

8.4 Predecessor computation for HO programs . . . . . . . . . . 167

8.5 Illustration for proof of soundness of predecessor computation for

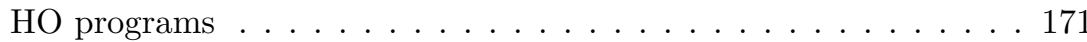




\section{List of Tables}

4.1 Experimental results of PhaserVerify . . . . . . . . . . . . . . 57

5.1 Reachability results for parameterized Phaser programs . . . . . . . 99

6.1 Experimental results of PACMAN . . . . . . . . . . 131

8.1 Experimental results of SyncV . . . . . . . . . . . . . . 173 



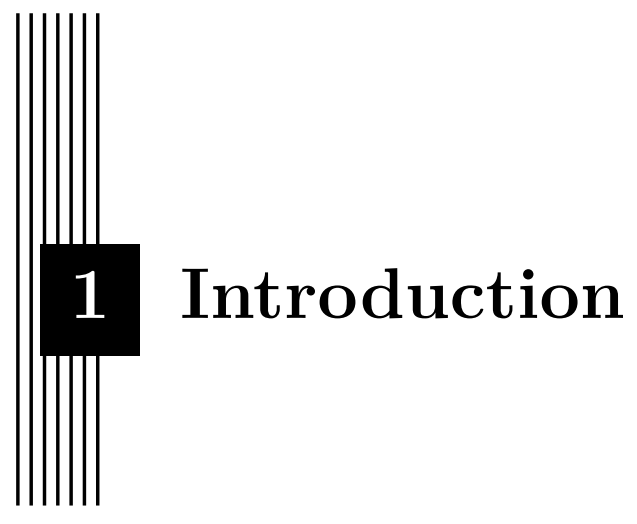

In this chapter, we present the motivation, objective, and the main contributions of the thesis. We start by discussing the importance and challenges of finding errors in concurrent programs. Then, we present a summary of related work. We also give a brief introduction to the contributions in this thesis.

\subsection{Motivation}

Although the first modern computers emerged in 1945 [120], until the mid1970s, the computers were large, expensive, and not connected. Since the mid-1970s, two major advances have changed the picture. Powerful microprocessors and computer networks were developed around this time [122] which contributed to the production of smaller, faster, and connectable computers.

Since the invention of the first multicore architectures in the 2000s, there have been several options available for concurrent execution of programs. There was also an increasing demand for concurrency. One of the reasons was that sequential programs were not expected to speed up significantly at this point. This is because efficiency of sequential programs relied directly on chip speed, which used to double every 18 months, but stopped doing so around this time.

Concurrency means several computations happen at the same time. There are different approaches to achieving concurrency, which are called concurrency models. We focus in this thesis on message passing and shared memory [116] concurrency models. 
In the message passing model, processes communicate with each other by passing messages over a network. Distributed systems [123] are an instance of this model. Distributed systems consist of processes running on autonomous and geographically dispersed computers. The processes interact with each other using a protocol to achieve a common goal. A distributed system appears to the user as a single coherent system.

In the shared memory model, the processes communicate with each other using shared variables. Each process may also have local variables that are not accessible to others. The concurrent use of shared resources can be a source of indeterminacy due to arbitrary interleaving of processes. This may lead to issues such as wrong results, deadlocks, or race conditions. In general, a synchronization mechanism is needed to control access to those resources. Barriers, semaphores, and locks are synchronization mechanisms that are widely used for this purpose. We will consider similar synchronization mechanisms in this work. For instance, we verify concurrent programs using Phasers [28, 41], a flexible synchronization construct that can capture both barriers and semaphores and that is implemented in modern languages such as Habanero Java.

Correctness properties for programs are usually divided into liveness properties and safety properties [117]. Safety properties intuitively mean that something bad never happens. Such properties include, for instance, the validity of assertions, race condition, and deadlock freedom. On the other hand, liveness properties indicate that something good will eventually happen. In this work, we focus on the verification of safety properties of synchronized concurrent programs.

Checking the correctness of concurrent programs is a complex task due to the interleavings of processes. Sometimes, violation of the correctness properties in such systems causes human or resource losses; therefore, it is crucial to check their correctness. For instance, several deficiencies in the development process of Therac- 25 software led to race conditions between concurrent processes that resulted in overdoses of radiation, causing the death of several cancer patients between 1985 and 1987 [129]. The first operational launch attempt of a space shuttle in the Aegis air-defense system failed due to a synchronization problem among its flight-control computers [95].

There are different approaches to analyzing the correctness of software. Such approaches differ in terms of guarantees they provide for the correctness of the target system. An analysis technique is sound for specific errors if a program is actually error-free, whenever the technique claims that it is. On the other hand, a technique is complete if a program is erroneous whenever the technique claims that it is. An analysis technique that is both sound and complete is called exact. Designing an algorithm to answer whether or not a program is correct would amount to solving the halting problem of a Turing machine. Therefore, automatic approaches cannot be exact and guaranteed to terminate in general. 
Two main approaches to software analysis are testing and formal verification. Testing is a dynamic analysis approach in which the correctness of a program is checked by providing test cases and validating their outputs. Although testing can help to discover many bugs at a low cost, it cannot prove the correctness of a program since it is not sound. As Dijkstra puts it, "Program testing can be used to show the presence of bugs, but never to show their absence" [49]. For example, the Ptolemy project [45] developed at Berkeley involved a case that illustrated the inability of testing for assuring the correctness of concurrent software. This project used Java threads for concurrent computation. After finalizing the implementation in early 2000, the software was reviewed by concurrency experts and tested extensively using regression tests that achieved 100 percent code coverage. Then, the system was widely used without any problem until it deadlocked in April 2004 [94]. However, if formal verification had been successfully used to check for deadlocks, this bug would not have been missed.

\subsection{Formal Verification}

The approach for proving software correctness is formal verification. Formal verification is a term for several techniques, including deductive verification, abstract interpretation, and model checking.

Deductive verification $[74,92, \mathbf{1 2 5}]$. In deductive verification, correctness of a program is established using axioms and proof rules. The primary approach among deductive verification techniques is the use of Hoare triples [73]. In this technique, the correctness of a program is proved by annotating different blocks of it (e.g., loops and function calls) by Hoare triples. A Hoare triple is of the form $\{S\} P\{Q\}$ where $P$ is the annotated program block, and $S$ is the precondition that $P$ must satisfy to ensure that the postcondition $Q$ holds after its execution, given that $P$ terminates. This approach builds correctness proofs incrementally and manually and may benefit partially from theorem provers to check the proofs. Deductive verification is a time-consuming and complicated task that needs to be performed by experienced logicians or mathematicians. Since it is a costly approach, it is usually used only for systems whose correctness is critical, such as security protocols. Deductive verification can be applied to infinite-state systems. However, this method is not fully automatic, and even if the system is correct, no limit can be put on the amount of time or memory required to obtain the correctness proof.

Abstract interpretation $[42,43,44]$ is a verification technique in which the program is analyzed using an abstract domain and abstract transformation. In fact, the concrete program is interpreted in the abstract domain using 
an abstraction function. Then, the abstract system is checked for correctness. Abstract interpretation techniques can check both liveness and safety properties. However, they often have difficulties with concurrency. Abstract interpretation has been used successfully in analyzing a wide range of programs, including the flight-control software of the Airbus A380, the world's largest passenger aircraft [118].

Model checking $[32,37,111]$ is a technique for formal verification that aims at automatically establishing the correctness of a program through an exhaustive search of the behavior of the system expressed in terms of temporal properties. Model checking is applied to a model of the program and is suitable for concurrent programs. It was initially introduced for verifying finite-state concurrent systems, and extending it to infinite-state systems is an active research area. The state-space of a system can be infinite because of infinite data types, recursion, or concurrency. The latter is the focus of this thesis.

Deductive verification, abstract interpretation, and model checking have different communities but have gradually exchanged ideas. For instance, model checking has used abstract interpretation ideas to abstract the statespace of the system $[14,36,51]$ in order to tackle the infinite state-space problem.

\subsection{Objective}

In this thesis, our focus is on the formal verification of parameterized systems. A system can be parameterized in different ways, such as the size of its arrays or the number of processes that execute it. In this work, we use the term parameterized systems to mean those systems in which the number of executing processes is not bounded a priori. In many concurrent algorithms and distributed protocols such as cloud architectures and sensor networks, an arbitrary number of processes run the program. For this reason, it is interesting to prove the correctness of parameterized systems regardless of the number of processes that execute them. This is called parameterized verification [2]. Formal verification is challenging per se, and making it parameterized exacerbates the situation even further.

Our goal is to provide fully-automatic and parameterized model checking techniques for establishing the correctness of certain classes of concurrent programs with regard to safety properties. We consider different synchronization paradigms from those implemented using shared program variables, to dynamic synchronization constructs such as Phasers, to static synchronization of distributed protocols. 


\subsection{Previous Work}

It is proved that the verification problem of parameterized programs is undecidable even for those involving simple processes [11, 103]. That is, there is no exact algorithm for checking the correctness of parameterized programs in general. However, parameterized verification has been an active line of research for decades, and extensive studies have been conducted to find decidable subclasses of the general problem. Pnueli and Zuck [130] survey the works on different techniques for parameterized verification. In general, two paths are taken as a remedy to undecidability: looking for restricted decidable subclasses of parameterized systems or developing sound, but necessarily incomplete verification techniques in the hope of finding proofs for the target systems.

\subsubsection{Decidable Subclasses of Parameterized Systems}

German and Sistla [64], and Emerson and Namjoshi [56] showed that parameterized verification is decidable for certain classes of synchronously communicating processes. Emerson and Kahlon [55] verify a general parameterized system that can contain several different types of processes. However, it requires all of the transition guards be either conjunctive or disjunctive. Abdulla et al. [2] proved that checking the reachability of an upward closed set of states for well-structured transition systems (WSTS) [2, 59], such as Petri nets [112], is decidable. Well-structured transition systems are infinite-state systems with a special partial order on their states, which is compatible with the transitions of the system. More precisely, the transitions of a WSTS are monotonic with respect to the order of its states (more details in Chapter 3).

\subsubsection{Beyond Decidable Subclasses}

Inductive techniques. The techniques in $[33,34,84]$ are based on induction on the number of processes in a network. Such methods are called inductive methods. Inductive methods usually require the user to provide inductive invariants of the system. Then, they check small instances of the system using those invariants and generalize them to be valid for any instance of the system. The method of invisible invariants [12, 109], on the other hand, automatically generates such induction assertions for safety and liveness verification of numerous systems that have finite data. In techniques based on induction, the challenge is to generalize the guessed invariant to find an invariant of the system that proves the property.

Deductive techniques. Owicki and Gries [106] introduced a deductive proof system for parallel programs using Hoare triples. This technique was later extended to parameterized programs in [105]. IronFleet [69] uses a mix- 
ture of TLA-style [92] and Hoare-logic to verify implementations of distributed protocols. Pandya and Joseph [108] introduced a logic similar to Hoare triples for safety verification of distributed protocols. In deductive techniques, an expert has to manually model the system and come up with the inductive invariants of the system and use a prover to validate them. Finding the right predicates and invariants for the proof is usually challenging.

Cut-off detection techniques. Empirical evidence suggests that parameterized systems often benefit from the small model property. Intuitively, the idea of the small model property is that in order to prove the correctness of a parameterized system, it is enough to analyze only a small number of the processes and their interactions. The upper bound on the number of processes to be analyzed is called the cut-off point. For many systems, such a point can be found statically before starting the verification process [80, 124]. For others, the cut-off point detection needs to be dynamic, that is, a dynamic approach starts with a small cut-off point and iteratively increments it in the hope of establishing the proof.

This idea gave rise to works in $[6,80,81,124]$. The methods presented in [6] and [81] have a dynamic approach to cut-off point detection, unlike the other ones which find it statically. The work in [99] has introduced a cut-off detection technique for a syntactically restricted class of distributed protocols.

Static cut-off detection approaches tend to restrict communication in the underlying systems. For instance, the work in [99] restricts the syntax of the guards used in the communication protocols, and the approach of [80] restricts itself to concurrent programs using locks.

Abstraction-based techniques. Such techniques try to reduce the size of the parameterized system by disregarding details that are unnecessary for the correctness proof. For example, counter abstraction [75, 97, 110] abstracts the system by counting the number of processes in each local state. The values of those counters, as well as a shared valuation, represent the state of the abstract system. Counter abstraction is not directly applicable to systems where the local states are infinite. Konnov et al. [79] introduced parametric interval abstraction as well as a new form of counter abstraction in which counters range over an abstract finite domain. They used this abstraction for parameterized verification of several distributed protocols [79, 86, 87, 88].

Symmetric concurrent programs can be analyzed by the predicate abstraction technique introduced in [51], which aims at reducing the state-space of each process to a finite space. This technique is based on [67], which introduced predicate abstraction for sequential programs. Environment abstraction [39] uses ideas from counter abstraction to enrich predicate abstraction.

Thread-modular analysis $[60,70]$ is another abstraction-based technique in which the state of a parameterized program is represented by the state 
of one process, as well as an environment which abstracts the interference of other processes. This is intended to address the exponential complexity in the control state of concurrent programs.

As opposed to most of the abstraction techniques that aim to reduce the system to a finite system, monotonic abstraction $[4,5,8]$ over-approximates a system into an infinite Well-Structured Transition System. Constrained monotonic abstraction (CMA) [3] allows for an iterative refinement scheme for monotonic abstraction (more details in Chapter 3 ).

In abstraction techniques, in general, it is challenging to find the right abstraction which is precise enough to not result in false alarms, but which disregards details that are irrelevant to the correctness property.

\subsection{Contributions}

We have made the following contributions for the verification of concurrent systems using complex synchronization constructs:

- We addressed the problem of automatically checking safety properties for Phaser programs. Phaser programs are concurrent programs that make use of a complex synchronization construct called Phasers. These are implemented in languages such as Habanero Java. We established the decidability of checking the violation of program assertions and the undecidability of checking deadlock-freedom.

- We again addressed the problem of automatically checking safety properties for Phaser programs, but, this time, we considered programs that might generate arbitrarily many processes and phasers (i.e., parameterized Phaser programs). We studied different formulations of the verification problem. We also proposed a procedure that is exact and guaranteed to terminate for some reachability problems, even for unbounded phases and arbitrarily many spawned processes.

For the verification of concurrent programs using integer shared variables for synchronization, we have made the following contributions:

- We proposed an approach to automatic verification of parameterized concurrent programs in which shared variables are manipulated by atomic transitions for the purpose of counting and synchronizing the spawned processes. For this purpose, we introduced counting predicates that mix counters referring to the number of processes that satisfy some given properties and variables that are directly manipulated by the concurrent processes. We then combined existing works on counter, predicate, and constrained monotonic abstraction and built a nested counterexample-based refinement scheme for establishing correctness. 
- We introduced Lazy Constrained Monotonic Abstraction for a more efficient exploration of well-structured abstractions of infinite-state nonmonotonic systems. We proposed several heuristics and assessed the efficiency of the proposed technique by performing extensive experiments using our open-source prototype tool.

For the verification of distributed protocols, we have made the following contribution:

- We proposed a sound but (in general) incomplete procedure for automatic verification of safety properties for a class of fault-tolerant distributed protocols described in the Heard-Of (HO for short) model. The HO model $[23,30]$ is a popular model for describing distributed protocols. HO protocols proceed in rounds where, at each round, each process sends a message to other processes, "hears" from a set of them, and updates its state. Fault descriptions are captured by stating constraints on the possible sets of processes and messages each process hears from (e.g., each process hears from at least half of the processes). This model uniformly describes distributed protocols in the presence of transmissionbased faults and captures a wide range of such faults being static or dynamic, transient, or permanent. We proposed a verification procedure that is guaranteed to terminate even for unbounded number of processes that execute the distributed protocol.

For most of the proposed techniques, we have implemented a prototype tool, which is publicly available and shows the application of the proposed method on a set of benchmarks. The results are reported in the thesis.

\subsection{Thesis Overview}

The following is an overview of the remaining chapters of the thesis.

In Chapters 2 and 3, we give some background and preliminaries. In Chapter 4, we present our first approach for verification of Phaser programs, which sets an upper bound on the number of processes in the program.

In Chapter 5, we push the verification of Phaser programs one step further and introduce another approach that conducts parameterized verification of Phaser programs. As opposed to the previous approach, this one does not set an upper bound on the number of processes and phasers.

In Chapter 6, we present a verification technique for parameterized verification of the safety of concurrent programs that implement complex synchronization schemes. This work combines counter, predicate, and monotonic abstractions. In Chapter 7, we investigate several methods for lazily refining monotonic abstraction. 
In Chapter 8, we introduce a novel approach for parameterized verification of fault-tolerant distributed protocols expressed in the Heard-Of model in the presence of different fault models. The approach is followed by proof of soundness and termination.

In Chapter 9, we conclude the thesis by summarizing the primary outcomes of our work and elaborating on potential directions for further development.

\subsection{Publication Overview}

The content of the thesis is based mainly on the following journal article and conference papers:

1. Zeinab Ganjei, Ahmed Rezine, Petru Eles, and Zebo Peng. "Safety verification of Phaser programs". In: Proceedings of the 15th International Conference on Formal Methods in Computer Aided Design, FMCAD 2017, Vienna, Austria, October 2017. IEEE, 2017, pp. 68-75.

2. Zeinab Ganjei, Ahmed Rezine, Ludovic Henrio, Petru Eles, and Zebo Peng. "On reachability in Parameterized Phaser programs". In: Proceedings of the 25th International Conference on Tools and Algorithms for the Construction and Analysis of Systems, TACAS 2019, Prague, Czech Republic, April 2019. Springer, 2019, pp. 299-315.

3. Zeinab Ganjei, Ahmed Rezine, Petru Eles, and Zebo Peng. "Abstracting and Counting Synchronizing Processes". In: Proceedings of the 16th International Conference on Verification, Model Checking, and Abstract Interpretation, VMCAI 2015, Mumbai, India, January 2015. Springer, 2015, pp. $227-244$.

4. Zeinab Ganjei, Ahmed Rezine, Petru Eles, and Zebo Peng. "Counting dynamically synchronizing processes". In: International Journal on Software Tools for Technology Transfer 18.5 (2016), pp. 517-534.

5. Zeinab Ganjei, Ahmed Rezine, Petru Eles, and Zebo Peng. "Lazy constrained monotonic abstraction". In: Proceedings of the 17th International Conference on Verification, Model Checking, and Abstract Interpretation, VMCAI 2016, St. Petersburg, FL, USA, January 2016. Springer, 2016, pp. 147-165.

6. Zeinab Ganjei, Ahmed Rezine, Petru Eles, and Zebo Peng. "Verifying safety of parameterized Heard-Of algorithms". In: Proceedings of the 8th International Conference on NETworked sYStems, NETYS 2020, Marrakech, Morocco, June 2020. Springer, 2020, pp. 209-226 .

The publications listed above correspond to the chapters of the thesis as follows. The work on Phaser programs verification presented in Paper 1 is 
covered in Chapter 4. The approach for parameterized verification of Phaser programs in Paper 2 is presented in Chapter 5. Paper 4 is the journal version of Paper 3. Its verification technique is introduced in Chapter 6 which targets general synchronization schemes using shared variables. Paper 5 introduces a method for optimizing constrained monotonic abstraction and is discussed in detail in Chapter 7. The work in Paper 6 on parameterized verification of a class of Heard-Of algorithms is covered in Chapter 8.

Lastly, the following conference paper uses the verification technique of Chapter 7 but is not discussed in the thesis:

1. Parosh Aziz Abdulla, Mohamed Faouzi Atig, Zeinab Ganjei, Ahmed Rezine, and Yunyun Zhu. "Verification of Cache Coherence Protocols wrt. Trace Filters". In: Proceedings of the Formal Methods in ComputerAided Design, FMCAD 2015, Austin, Texas, USA, September 2015, IEEE, pp. 9-16. 


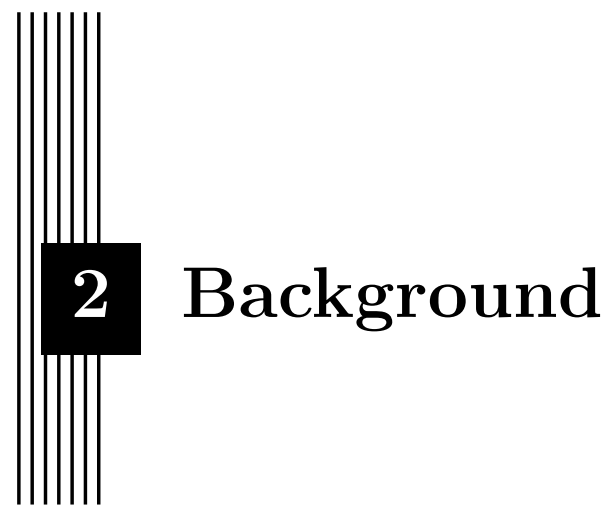

This work focuses on extending abstraction and model checking of infinitestate systems to new classes of concurrent and distributed programs. In this chapter, we recall several synchronization mechanisms that are widely used in concurrent programs, as well as ideas from abstraction and model checking of safety properties.

\subsection{Synchronization Mechanisms}

In the following, we recall three widely-used synchronization mechanisms in concurrent systems, namely, barriers, semaphores, and locks. We also briefly introduce Phasers, which are a modern and dynamic synchronization paradigm.

A barrier for a group of processes means that any process must stop at this point and cannot proceed until all other processes reach the barrier. Barriers can be either static or dynamic. With static barriers, the number of processes that have to stop at the barrier is fixed and is usually set at the time of the barrier's instantiation. On the other hand, with dynamic barriers, processes can be dynamically added as participants in the barrier or can remove themselves from the synchronization. Dynamic barriers, however, are more challenging to implement than static barriers.

A semaphore [116] is a non-negative integer variable on which only two atomic operations - wait and signal - can be performed. The wait operation decrements the value of the semaphore variable by 1 if it is positive. 
Otherwise, the process that executes wait is blocked. The signal operation increments the value of the semaphore variable by 1 . The supporting environment must provide the atomicity of the semaphore implementation. A semaphore with a maximum value of 1 is called a binary semaphore.

Locks [116] are another synchronization construct that are usually implemented by binary semaphores and have two operations, namely, acquire (instead of wait) and release (instead of signal).

Phasers are a synchronization mechanism introduced with the Habanero Java API $[28,41]$. They implement a flexible synchronization paradigm, which is general enough to model static and dynamic barriers and semaphores. Intuitively, a phaser associates two phases (hereafter wait and signal phases) with each registered process. Apart from creating phasers and registering each other to them, processes can individually issue wait and signal commands to a phaser they are registered with. Intuitively, signal commands are used to inform other registered processes that the issuing process is done with its signal phase. The wait command is instead used to check whether all registered processes are done with the wait phase of the issuing process. This command may get blocked by a process that has not yet finished the corresponding phase.

\subsection{Model Checking}

Model checking $[13,32,37,111]$ is a fully automated method for analyzing systems modeled as Kripke structures or transition systems. Model checking is widely used for verification of hardware and software systems. It conducts an exhaustive search on the behavior of a system model to determine whether or not the desired specifications hold. To perform Model Checking, first, correctness properties or specifications of systems are written in temporal logic. Then, a decision procedure determines whether the model satisfies those properties. If not, the model checker outputs a counterexample that witnesses that the model violates the correctness property. Model checking is based on the algorithmic exploration of state-spaces.

\subsubsection{Transition Systems}

Transition systems are often used to capture the semantics of software systems. A transition system is a tuple $\mathcal{T S}=\left(\mathrm{C}, \mathrm{C}_{\text {init }}, \mathrm{T}, \rightarrow\right)$ where $\mathrm{C}=\left\{\mathrm{c}_{1}, \mathrm{c}_{2}, \ldots\right\}$ is a set of configurations of the system (for a program, configurations typically correspond to the values of all the program variables at a particular time), $\mathrm{C}_{\text {init }} \subset \mathrm{C}$ is the set of initial configurations (i.e., configurations from which the program may start execution), and $\rightarrow \subseteq \mathrm{C} \times \mathrm{T} \times \mathrm{C}$ is a set of transitions between configurations. A run $\rho$ is an alternating sequence of configurations and transitions starting with a configuration, $\mathrm{c}_{0} ; \mathrm{t}_{0} ; \mathrm{c}_{1} ; \mathrm{t}_{1} ; \ldots ; \mathrm{c}_{n}$, such that $\mathrm{c}_{0}$ is an initial configuration and for each $i \geq 0$, we have $\mathrm{c}_{i} \stackrel{\mathrm{t}_{i}}{\longrightarrow} \mathrm{c}_{i+1}$. 
We use $\stackrel{*}{\rightarrow}$ for the reflexive and transitive closure of $\rightarrow$. We write $c \stackrel{*}{\rightarrow} c^{\prime}$ to mean that $c^{\prime}$ is reachable from c. A configuration is said to be reachable if it is reachable from some initial configuration $\mathrm{C}_{\text {init }}$ in $\mathrm{C}_{\text {init }}$.

A transition system $\mathcal{T} \mathcal{S}$ can have an infinite set of configurations. We say that such a system is an infinite transition system.

Given a transition system $\mathcal{T S}=\left(\mathrm{C}, \mathrm{C}_{\text {init }}, \mathrm{T}, \rightarrow\right)$, and a target configuration $\mathrm{c}_{\text {trgt }} \in \mathrm{C}$, the reachability problem asks whether the target configuration $\mathrm{c}_{\text {trgt }}$ is reachable in $\mathcal{T S}$. Also, the coverability problem asks whether some configuration $\mathrm{c} \in \mathrm{C}$ is reachable in $\mathcal{T S}$ such that $\mathrm{c}_{\text {trgt }} \leq \mathrm{c}$ where $\leq$ is a partial order on configurations. Extending a transition system with a labeling function $\mathcal{L}: \mathrm{C} \rightarrow 2^{\mathrm{A}}$ that labels its configurations with respect to a non-empty set of atomic propositions A results in a Kripke structure [89].

\subsubsection{Specification}

The specification of a system states the properties the system must satisfy. Such properties are usually expressed in terms of some logical formalism. The underlying logic determines the expressive power of the specification; for instance, propositional logic has less power than temporal logics. Temporal logics are formalisms that describe temporal (as in sequences) behaviors of reactive systems. Two popular temporal logics are computation-tree logic (CTL) and linear-time logic (LTL) [37]. In LTL (also called propositional temporal logic), one can encode formulae about the future of paths; for instance, a formula that specifies some property will eventually always hold on all paths. In CTL, on the other hand, the model of time is a tree-like structure (the computation tree) in which the future is not determined; there are different paths in the future, any one of which might be an actual path that is realized. A computation tree is obtained by unfolding the Kripke structure. The root of each computation tree is some initial configuration $\mathrm{c}_{0}$. CTL can specify, for instance, that there exists a path such that from its second state onward, some property holds.

Two classes of properties are often considered for showing the correctness of a system, i.e., liveness properties and safety properties. In this thesis, we focus on verifying safety properties for concurrent and distributed systems.

\section{Safety Properties}

Intuitively, a safety property expresses that "nothing bad happens" during the execution of the system. In many systems, Safety properties can be captured by determining a set of bad configurations and asserting that the system does not end up in one of those. A system is considered to be unsafe if some of its bad configurations are reachable. Otherwise, the system is called safe. 


\subsubsection{Decidability}

The model checking problem $M \vDash S$ is the decision problem, which asks whether the model $M$ (often given as a Kripke structure) satisfies some temporal property $S$ characterizing configurations of the Kripke structure. This problem is decidable for a set of models and properties if there is a terminating procedure that can answer it for any model and property in the considered sets. In this work, where we consider safety propertyies, this boils down to deciding the reachability of sets of configurations.

\subsection{Abstraction}

With abstraction, the details that are not relevant to the property to be checked are removed (abstracted away), which usually reduces the size of the state-space. This approach is used widely in model checking to tackle the state-space explosion problem. To perform abstraction, one has to implicitly build the abstract transition system from a high-level description of the system [37] and refine it if needed, for example, using the Counterexample-Guided Abstraction Refinement technique (introduced in Section 2.3.1).

In this thesis, we use Existential Abstraction [36]. A transition system $\hat{\mathcal{T S}}=\left(\hat{\mathrm{C}}, \mathrm{C}_{\text {init }}, \hat{\mathrm{T}}, \hat{\rightarrow}\right)$ is an existential abstraction of $\mathcal{T} \mathcal{S}=\left(\mathrm{C}, \mathrm{C}_{\text {init }}, \mathrm{T}, \rightarrow\right)$ where:

- the abstraction of any initial configuration in $\mathrm{C}_{\text {init }}$ is an initial configuration of the abstract system; and

- if two configurations are related with $\rightarrow$, their abstractions must be related with $\hat{\rightarrow}$.

A concretization relation indicates a set of concrete configurations for each abstract configuration. An abstract model constructed using existential abstraction is sound for reachability problems. That is, if some abstract set of configurations is not reachable in the abstract system, then its concretization is not reachable in the concrete one either. Such an abstract model is an over-approximation of the concrete model. That is, it might allow more behavior than the concrete model. Hence, if the abstract system is safe, so is the concrete one. An example of such abstraction is Predicate Abstraction (see Section 6.5.1).

\subsubsection{Counterexample-Guided Abstraction Refinement}

The abstract models obtained through existential abstraction usually contain less information than the original ones. Therefore, model checking the abstract model might produce incorrect results, such as spurious traces to error. The framework of CounterExample-Guided Abstraction Refinement 
(CEGAR) [35] is used for refining an abstraction to eliminate false positives and hence, make the abstraction more precise.

The CEGAR framework consists of the following steps:

1. Given a model $M$ and a specification $S$, generate an initial abstract model $\hat{M}$.

2. Model check $\hat{M}$ with respect to the specification $S$. If $\hat{M}$ satisfies $S$, then $M$ also satisfies it and conclude. If a counterexample $\hat{\rho}$ is found, check whether the trace $\hat{\rho}$ is also possible in the concrete model. If this is the case, conclude that $M$ does not satisfy $S$ and stop. Otherwise, $\hat{\rho}$ is spurious. Continue to step 3.

3. Refine the abstract model so that the counterexample $\hat{\rho}$ will not be included in the refined abstract model. Return to step 2.

As stated earlier, each abstract configuration usually represents a set of concrete configurations. For refinement, usually, one or more abstract configurations are partitioned. 



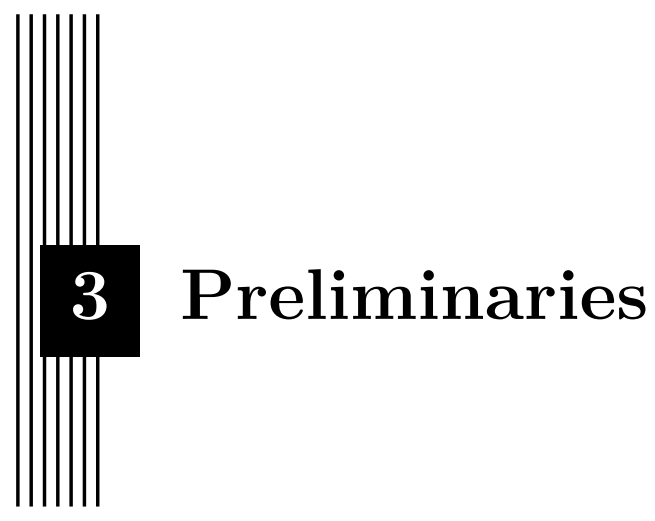

In this chapter, we introduce some notations, definitions, and theorems used in the subsequent chapters. We present counter machines, well-structured transition systems, a reachability analysis procedure, monotonic abstraction, and its refinement.

\subsection{Preorders, Configurations, and Constraints}

The binary relation $\leq$ is a preorder or quasi-ordering over a set $A$, if it is reflexive and transitive. A quasi-ordering $\leq$ is a well-quasi-ordering $(\mathcal{W Q O})$ over a set $A$ if there is no infinite sequence $a_{0}, a_{1}, \ldots$ of elements in $A$ such that $a_{i} \npreceq a_{j}$ for all $i<j$. We often capture possibly infinite sets of configurations with a symbolic representation $\varphi$ and call it a constraint. A constraint can, for instance, be the upward closure of some configuration $c$ with respect to a quasi-ordering $\leq$, or a logical formula that characterizes the configurations in the set. Given a configuration $\mathrm{c}$ and a constraint $\varphi$, we say that $\mathrm{c}$ satisfies $\varphi$, and write $c \vDash \varphi$, if $c$ satisfies conditions imposed by $\varphi$. We define the set of configurations denoted by (or characterized by) $\varphi$ as $[\varphi]=\{c \mid c \vDash \varphi\}$.

The image computation function next $t_{t}(\varphi)$ gives the set of all of the images of $\varphi$ with respect to the transition t. $\left[\operatorname{nnext}_{t}(\varphi)\right] \cup[[\varphi]]$ can be used to over-approximate the set $\left\{c^{\prime} \mid c^{\prime} \stackrel{t}{\rightarrow} c\right.$ and $\left.c \in[\varphi \varphi]\right\}$ of predecessors, or the set $\left\{c^{\prime} \mid c \stackrel{t}{\rightarrow} c^{\prime}\right.$ and $\left.c \in[[\varphi]]\right\}$ of successors of configurations denoted by $\varphi$. We abuse notation and use next $t_{t}(\Phi)$ on a set of constraints as well. 
We say a preorder $\subseteq$ on constraints is sound if $\varphi \sqsubseteq \varphi^{\prime}$ implies $\left[\left[\varphi^{\prime}\right] \subseteq[[\varphi]]\right.$. Also, an image computation function next $\operatorname{ta}_{\mathrm{t}}(\varphi)$ used to approximate successor (respectively, predecessor) configurations with respect to transition $t$ is said to be sound if for any arbitrary configuration $\mathrm{c} \in[[\varphi]]$ where $\mathrm{c} \stackrel{\mathrm{t}}{\rightarrow} \mathrm{c}^{\prime}$ (respectively, $\left.\mathrm{c}^{\prime} \stackrel{\mathrm{t}}{\rightarrow} \mathrm{c}\right), \mathrm{c}^{\prime}$ is in $\left[\operatorname{next}_{\mathrm{t}}(\varphi)\right] \cup[[\varphi]]$. On the other hand, $\operatorname{next}_{\mathrm{t}}(\varphi)$ is complete if for all configurations $c^{\prime}$ denoted by next $t_{t}(\varphi)$, there exists a configuration $c \in\left[[\varphi]\right.$, where $\mathrm{c} \stackrel{\mathrm{t}}{\rightarrow} \mathrm{c}^{\prime}$ (respectively, $\mathrm{c}^{\prime} \stackrel{\mathrm{t}}{\rightarrow} \mathrm{c}$ ). We say that next $\mathrm{t}_{\mathrm{t}}$ is exact when it is both sound and complete. We always require soundness of next $t_{t}$ and $ᄃ$.

With respect to a next function which is clear from the context, we define a trace $\rho=\varphi_{0} ; \mathrm{t}_{1} ; \ldots ; \mathrm{t}_{n} ; \varphi_{n}$ to be an alternating sequence of constraints and transitions that starts with a constraint. Moreover, for $i: 0 \leq i<n$, $\varphi_{i+1} \in \operatorname{next}_{\mathrm{t}_{i+1}}\left(\varphi_{i}\right)$.

\subsection{Counter Machines}

A counter machine is a tuple $\mathrm{M}=\left(\mathcal{Q}, \mathcal{C}, \mathrm{T}, \mathcal{Q}_{\text {init }}, \mathrm{q}_{\text {trgt }}\right)$ where $\mathcal{Q}$ is a finite set of states of the machine, $\mathcal{C}$ is a finite set of counters (i.e., variables ranging over the natural numbers $\mathbb{N}$ ), $\mathrm{T}$ is a finite set of transitions, $\mathcal{Q}_{\text {init }} \subseteq \mathcal{Q}$ is a set of initial states of the machine, and $\mathrm{q}_{\text {trgt }}$ is a state in $\mathcal{Q}$. A transition $\mathrm{t}$ in $\mathrm{T}$ is of the form $\left(\mathrm{q}: \mathrm{op}: \mathrm{q}^{\prime}\right)$ where $\operatorname{src}(\mathrm{t})=\mathrm{q}$ is the source state, $\operatorname{dst}(\mathrm{t})=\mathrm{q}^{\prime}$ is the destination state, and the operation op is either the identity operation nop, or a guarded command grd $\Rightarrow$ cmd. A guard grd is a predicate on counters. $\mathrm{e}$ is an expression over $\mathcal{C}$ and a command $\mathrm{cmd}$ is a multiple assignment $c t_{1}, \ldots, c t_{n}:=e_{1}, \ldots, e_{n}$ that involves assignments of values of expressions $\mathrm{e}_{1}, \ldots, \mathrm{e}_{n}$ to pairwise different counters $c t_{1}, \ldots c t_{n}$ in $\mathcal{C}$.

A machine configuration $\mathrm{c}$ is a pair $(\mathrm{q}, \mathbb{C})$ where $\mathrm{q}$ is a state in $\mathcal{Q}$ and $\mathbb{C}$ is a mapping $\mathcal{C} \rightarrow \mathbb{N}$. $\quad \mathrm{C}$ is the set of all such configurations. The set of initial machine configurations $\mathrm{C}_{\text {init }}$ consists of those configurations from which the execution of the system may start. More specifically, $\mathrm{C}_{\text {init }}$ contains configurations of the form $(\mathrm{q}, \mathbb{C})$ where $\mathrm{q} \in \mathcal{Q}_{\text {init }}$ and $\mathbb{C}$ maps every counter to zero. The image computation function $\underset{\mathrm{M}}{\mathrm{t}} \subseteq \mathrm{C} \times \mathrm{T} \times \mathrm{C}$ is defined as follows: $(\mathrm{q}, \mathbb{C}) \underset{\mathrm{M}}{\stackrel{\mathrm{t}}{\rightarrow}}\left(\mathrm{q}^{\prime}, \mathbb{C}^{\prime}\right)\left(\right.$ or $(\mathrm{q}, \mathbb{C}) \stackrel{\mathrm{t}}{\rightarrow}\left(\mathrm{q}^{\prime}, \mathbb{C}^{\prime}\right)$ for short in case $\mathrm{M}$ is clear from the context) iff $\operatorname{src}(\mathrm{t})=\mathrm{q}, \mathbb{C} \vDash \operatorname{grd}, \operatorname{dst}(\mathrm{t})=\mathrm{q}^{\prime}$, and $\mathbb{C}^{\prime}\left(\mathrm{ct}_{i}\right)=\mathrm{e}_{i}[\mathbb{C}]$ for $i: 1 \leq$ $i \leq|\mathcal{C}|$, where $e_{i}[\mathbb{C}]$ is the result of evaluating the expression $e_{i}$. Considering a preorder $\leq$ on the machine configurations, a set of configurations $\mathrm{C}$ is said to be upward-closed if for every configuration $c \in C, c \leq c^{\prime}$ implies $c^{\prime} \in \mathrm{C}$. An $\mathrm{M}$ run $\rho_{\mathrm{M}}$ is a sequence $\left(\mathrm{q}_{0}, \mathbb{C}_{0}\right) ; \mathrm{t}_{1} ; \ldots ; \mathrm{t}_{n} ;\left(\mathrm{q}_{n}, \mathbb{C}_{n}\right)$. This run is feasible if $\left(\mathrm{q}_{0}, \mathbb{C}_{0}\right)$ is initial and $\left(\mathrm{q}_{i}, \mathbb{C}_{i}\right) \stackrel{\mathrm{t}_{i+1}}{\longrightarrow}\left(\mathrm{q}_{i+1}, \mathbb{C}_{i+1}\right)$ for $i: 0 \leq i<n$. We write $\rightarrow$ for $\cup_{\mathrm{t} \in \mathrm{T}} \stackrel{\mathrm{t}}{\rightarrow}$. 
Counter machines are a popular model for parameterized systems in which both the shared and local configurations of each process are finite. Intuitively, each counter can represent a specific process-local configuration. The value of the counters can capture the number of processes in different local configurations. Moreover, the counter machine state can model the shared configuration of the system, e.g., the valuation of the shared variables. Violation of mutual exclusion can be easily captured for such a system by representing the critical section with a counter that has a value of at least two.

\subsubsection{Safety Problem of a Counter Machine}

The safety property of a counter machine can be formulated in terms of a set $\mathrm{C}_{b a d}$ of bad configurations. These are configurations that should not occur during the execution of the system. Thus, checking the safety property can be reduced to checking the reachability of the set of bad configurations.

If the set $\mathrm{C}_{b a d}$ is upward-closed, checking the safety property amounts to checking the reachability of an upward-closed set, which is called the coverability problem. For instance, the set $\mathrm{C}_{b a d}$ that formulates violation of the mutual exclusion property is an upward-closed set and checking it results in a coverability problem.

The state reachability or control reachability problem of the counter machine for target state $\mathrm{q}_{\text {trgt }}$ is to decide whether there is an M-feasible run $\left(\mathrm{q}_{0}, \mathbb{C}_{0}\right) ; \mathrm{t}_{1} ; \ldots ; \mathrm{t}_{n} ;\left(\mathrm{q}_{n}, \mathbb{C}_{n}\right)$ such that $\mathrm{q}_{n}=\mathrm{q}_{\text {trgt }}$. We say that the run covers the state $\mathrm{q}_{n}$. The state reachability of a counter machine is a coverability problem.

We often use classical results about 2-counter Minsky machines and vector addition systems with reset arcs in this work. In the following section we introduce these counter machines and the results that we use.

\subsubsection{2-counter Minsky Machine}

Let $\mathrm{M}=\left(\mathcal{Q}, \mathcal{C}, \mathrm{T}, \mathcal{Q}_{\text {init }}, \mathrm{q}_{\text {trgt }}\right)$ be a counter machine with two counters $\mathcal{C}=$ $\left\{c t_{1}, c t_{2}\right\}$ initially at zero. Let the transitions in $\mathrm{T}$ be either an increment ( $\mathrm{q}:$ true $\left.\Rightarrow c t_{i}:=c t_{i}+1: \mathrm{q}^{\prime}\right)$, a decrement $\left(\mathrm{q}: \mathrm{ct}_{i} \geq 1 \Rightarrow c \mathrm{t}_{i}:=c \mathrm{t}_{i}-1: \mathrm{q}^{\prime}\right)$ or a test for zero $\left(\mathrm{q}: \mathrm{ct}_{i}=0 \Rightarrow \mathrm{nop}: \mathrm{q}^{\prime}\right)$ for $i \in\{1,2\}$. This is called a 2-counter Minsky machine.

Theorem 1. The state reachability problem of a 2-counter Minsky machine is undecidable [103].

The idea is to show that for every Turing machine there is a 2-counter Minsky machine that simulates it. The halting problem of a Turing machine can be reduced to the state reachability problem of a 2-counter Minsky machine. One way is to perform the following encoding with three steps. First, a Turing machine can be simulated by a finite-state machine equipped with 
two stacks. Then, a stack can be simulated by two counters. Finally, four counters can be simulated by two counters.

We will often use this result to show that fundamental verification problems of a concurrent system are undecidable. We can reduce the state reachability problem of a Minsky machine into its reachability problem. Hence, the reachability problem of Minsky machines is also undecidable.

\subsubsection{Vector Addition Systems}

A Vector Addition System (VAS) is a counter machine $\mathrm{M}=$ $\left(\mathcal{Q}, \mathcal{C}, \mathrm{T}, \mathcal{Q}_{\text {init }}, \mathrm{q}_{\text {trgt }}\right)$ with only the increment $\left(\mathrm{q}:\right.$ true $\left.\Rightarrow \mathrm{ct}_{i}:=\mathrm{ct}_{i}+1: \mathrm{q}^{\prime}\right)$ and decrement $\left(\mathrm{q}: \mathrm{ct}_{i} \geq 1 \Rightarrow c t_{i}:=\mathrm{ct}_{i}-1: \mathrm{q}^{\prime}\right)$ operations. VAS with reset arc (or reset-VAS) is a VAS extended with the reset operation, ( $\mathrm{q}:$ true $\left.\Rightarrow \mathrm{ct}_{i}:=0: \mathrm{q}^{\prime}\right)$ for $i \in\{1, \ldots, n\}$.

Theorem 2. The coverability problem of VAS with reset arcs is decidable [54].

The argument for Theorem 2 is presented in Section 3.3, where we describe a reachability procedure that can be shown to be exact and to terminate for coverability of WSTS (Section 2) in general and VAS with reset arcs in particular.

Theorem 3. The reachability problem of VAS with reset arcs is undecidable [54].

A 2-counter Minsky machine can be modeled as a reset-VAS. The intuition is that for every counter ct in the Minsky machine, the encoded VAS will contain two counters, ct and $c^{\prime}$, and their values must always be equal. Increments and decrements will update both ct and ${ }^{\prime} t^{\prime}$ in the same way. Each test for zero of ct will be modeled by resetting $c t^{\prime}$. In this way, the first time ct is tested for zero in the Minsky machine when ct $>0$, the values of ct and ct $^{\prime}$ in the VAS will become different, which can never be recovered. In the Minsky machine, this step is not possible since ct $>0$.

We will use this result to show that checking deadlock freedom for certain phaser programs is undecidable in general.

\subsection{Working-List Procedure}

A consequence of Theorem 1 is that model checking infinite transition systems is undecidable in general. In the procedure "explore", we adopt a workinglist reachability analysis to search the infinite state-space. The intuition is to adopt constraints instead of configurations in the procedure "explore" below. 


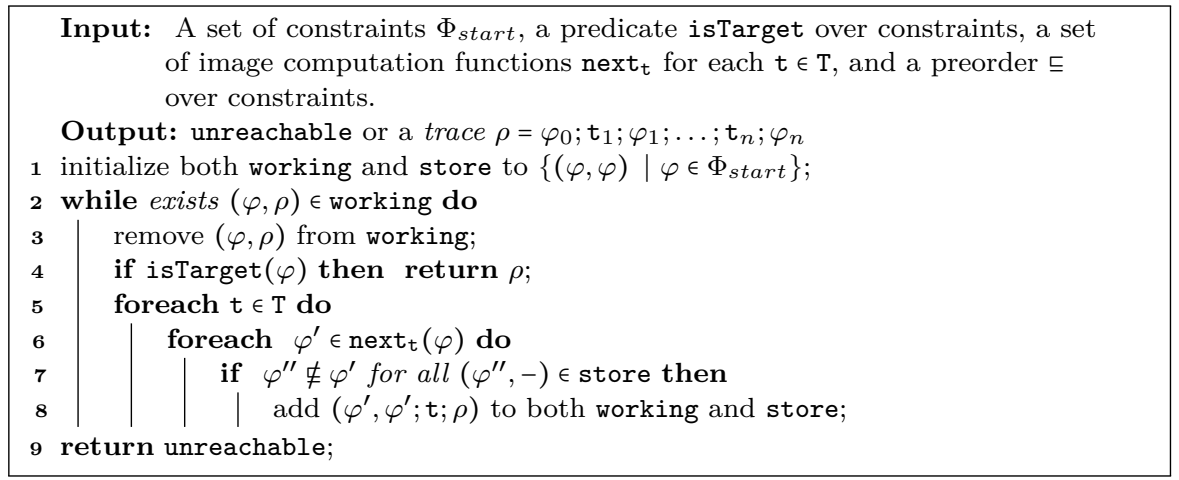

Procedure explore $\left(\Phi_{\text {start }}\right.$, isTarget, T, next, ㄷ). A working-list procedure to check whether some target configuration can be obtained from one of the constraints in $\Phi_{\text {start }}$ using the image computation functions next $_{t}$ for each $t \in T$, and the preorder $\sqsubseteq$ on the constraints. The predicate isTarget determines whether a constraint denotes a target configuration.

The procedure starts from a set of constraints $\Phi_{\text {start }}$. Its goal is to check if some constraint for which isTarget evaluates to true can be obtained from one of the constraints in $\Phi_{\text {start }}$ using the image computation functions next $t_{t}$ for each $t \in \mathrm{T}$. The procedure uses a set working whose elements are pairs $(\varphi, \rho)$ where each pair consists of a constraint $\varphi$ and the trace traversed from some $\varphi_{\text {start }} \in \Phi_{\text {start }}$ to $\varphi$. Such constraints can be obtained from a $\varphi_{\text {start }}$ with a succession of next $=\bigcup_{t \in T}$ next $_{t}$ and from them the procedure will continue the search. The set store, on the other hand, stores all the encountered $(\varphi, \rho)$ during the analysis unless the encountered pair has a constraint $\varphi^{\prime}$ that is larger (with respect to ㄷ) than some constraint that is already in store.

The procedure takes a possibly infinite loop (line 2) in which during each iteration, it first removes a pair $(\varphi, \rho)$ from working (line 3$)$. Then, it checks whether isTarget evaluates to true for $\varphi$ (line 4). If so, it returns the trace $\rho$ as a possible reachability witness. Otherwise, the procedure computes images of $\varphi$ with respect to each of the system transitions (line 6). For each newly generated constraint $\varphi^{\prime}$, the procedure checks whether another constraint $\varphi^{\prime \prime} \sqsubseteq \varphi^{\prime}$ had been previously encountered (line 7 ). In this case, we say that $\varphi^{\prime}$ is subsumed by $\varphi^{\prime \prime}$. Otherwise, a new pair constructed with $\varphi^{\prime}$ and the trace traversed to it is added to both working and store sets (line 8). The procedure terminates when all of the newly generated constraints are subsumed by constraints that had been generated in previous iterations, which are stored in store. In such a case, working will eventually be empty, and store would be the set of constraints that denote configurations reachable from some $\varphi_{\text {start }}$. Under suitable assumptions, one can show that a target configuration is reachable from a $\varphi_{\text {start }}$ iff a constraint in store denotes it. 
The procedure "explore" can be used for forward or backward explorations. In forward exploration, $\Phi_{\text {start }}$ is a set of constraints that denote the set of initial configurations $\mathrm{C}_{\text {init }}$, next $(\varphi)$ computes or approximates successors of the denotation of $\varphi$, and isTarget $(\varphi)$ checks whether a bad configuration in $\mathrm{C}_{b a d}$ is denoted by $\varphi$. In backward exploration, $\Phi_{\text {start }}$ is a set of constraints that denote a set of bad configurations $\mathrm{C}_{b a d}$, next $(\varphi)$ computes or approximates predecessors of denotations of $\varphi$, and isTarget $(\varphi)$ checks whether a configuration in $\mathrm{C}_{\text {init }}$ is denoted by $\varphi$.

We say that $ᄃ$, isTarget and next $t_{\mathrm{t}}$ are effective, if the following conditions are satisfied:

- For every pair of constraints $\varphi_{1}$ and $\varphi_{2}$, it is possible to check whether $\varphi_{1} \subseteq \varphi_{2}$,

- For every constraint $\varphi$, isTarget $(\varphi)$ can be evaluated,

- For every constraint $\varphi$ and $t$, the set next $t_{t}(\varphi)$ can be computed.

If $ᄃ$, isTarget and next $t_{\mathrm{t}}$ are effective, we say the procedure "explore" is effective.

In the following, we show some results about the procedure "explore".

Corollary 1. Let working w $_{k}$ and store $_{k}$ be the sets working and store obtained at line 2 of the $k^{\text {th }}$ iteration of the loop in procedure "explore". Suppose constraintOf $\left(\right.$ store $\left._{k}\right)$ gives the constraints of the pairs stored in store . $_{\text {. }}$

(a) store $_{k}$ contains $\Phi_{\text {start }}$

(b) working $_{k}$ is a subset of store $k$

(c) for each element $(\varphi, \rho)$ of store $_{k}$ such that $(\varphi, \rho) \notin$ working $_{k}$ and for each transition $\mathrm{t} \in \mathrm{T}$, every image constraint $\varphi^{\prime}$ in $\operatorname{next}_{\mathrm{t}}(\varphi)$ or some entailing constraint $\varphi^{\prime \prime} \sqsubseteq \varphi^{\prime}$ is in constraint0f( store $\left._{k}\right)$

Proof. We call a pair $(\varphi, \rho)$ of constraint and trace an element for short.

Base case: $k=0$. At line 1 of the procedure "explore", an element containing the starting constraint is the only element added to both store $\mathrm{s}_{0}$ and

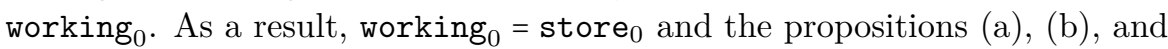
(c) hold for $k=0$.

Suppose the propositions hold up to $k$. We show they hold for $k+1$.

For proposition (a), note that an element that is added to store is never removed from it, and for proposition (b), note that whenever an element is

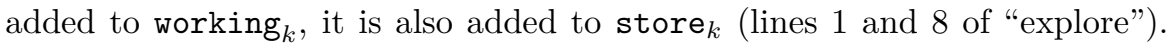
The intuition of proposition (c) is as follows. The algorithm always picks an element $(\varphi, \rho) \in$ working $_{k}$ and computes its images with respect to all transitions. Therefore, for any element $\left(\varphi^{\prime}, \rho^{\prime}\right) \notin$ working $_{k}$, the images must 
be subsumed by elements in store $_{k}$. The only places where store $_{k}$ and working $_{k}$ are manipulated, are at lines 1, 3, and 8 of "explore". At line

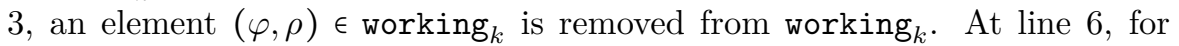
each transition $\mathrm{t} \in \mathrm{T}$, next $\tan _{\mathrm{t}}(\varphi)$ is computed, then all elements $\left(\varphi^{\prime}, \varphi^{\prime} ; \mathrm{t} ; \rho\right)$ are added to working ${ }_{k}$ and store $_{k}$, in case no other element with constraint $\varphi^{\prime \prime} \sqsubseteq \varphi^{\prime}$ already exists in store ${ }_{k}$. Thus, the images of any constraint removed from working ${ }_{k}$ are entailed by elements in store $_{k}$.

We show the following theorems for the backward "explore". The forward case is similar.

Theorem 4 (Soundness of "explore"). If next, isTarget, and 5 are sound, and the procedure "explore" returns unreachable, then no configuration c denoted by $\Phi_{\text {start }}$ is reachable from any configuration $\mathrm{c}_{\text {init }} \in \mathrm{C}_{\text {init }}$.

Proof. By soundness of $\sqsubset$, only those constraints are not added to store that denote configurations already denoted by constraintof(store). Moreover, by the soundness of next $t_{t}$, the images of a constraint together with the constraint itself denote all of its predecessor configurations.

Suppose the algorithm returns unreachable. Then, at some iteration, there is no element $(\varphi, \rho)$ in working. Combined with propositions (a), (b) and (c) of Corollary 1, we have that after termination, $\bigcup_{t \in T}$ next $_{t}($ constraintOf $($ store $)) \subseteq$ constraintOf(store). Hence, all configurations that can reach $\Phi_{\text {start }}$ are denoted by constraintOf(store) and they do not contain any configuration in $\mathrm{C}_{\text {init }}$ by soundness of isTarget.

Definition 1. We say isTarget is complete if whenever isTarget $(\varphi)$ evaluates to true, $\varphi$ denotes some target configuration.

Theorem 5. Assume "explore" is a backward exploration. If next and isTarget are complete, and the procedure "explore" returns a counterexample, that counterexample is real.

Proof. Assume that the procedure returns a trace $\rho=\varphi_{n} ; \mathrm{t}_{n} \ldots \mathrm{t}_{1} ; \varphi_{\text {start }}$, then, the test at line 4 ensures that isTarget $\left(\varphi_{n}\right)=$ true for some $\left(\varphi_{n}, \rho\right)$. We proceed by contradiction. If the trace is not real, there is some $0 \leq i<$ $n$, where there is some configuration $c^{\prime \prime}$ in $\left[\left[\varphi_{i+1}\right]\right]$ which is not in the set $\left\{c^{\prime} \mid c^{\prime} \rightarrow c\right.$ and $\left.c \in\left[\left[\varphi_{i}\right]\right]\right\}$. Intuitively, $c^{\prime \prime}$ causes the spurious trace. This contradicts completeness of next.

Theorem 6. If the preorder $\subseteq$ is a well-quasi-ordering $(\mathcal{W Q O})$, then the procedure "explore" terminates if $\sqsubseteq$, next, and isTarget are effective. 
Proof. Assuming ㄷ, next and isTarget are effective, the only source of nontermination can be the while loop in line 2 if the set working never becomes empty. Suppose there is an infinite sequence of constraints as $\varphi_{0}, \varphi_{1} \ldots$ obtained from working in different iterations of the while loop at line 2 . We show that for any $0 \leq i<j, \varphi_{i} \notin \varphi_{j}$. This is because (1) working is a subset of store according to $(b),(2)$ an element is never removed from store, and (3) an element is added to store only if no smaller element with respect to 드 is already there (lines 1 and 8 ). Such a sequence would therefore violate well-quasi-orderedness.

Definition 2 (Well-Structured Transition Systems). A well-structured transition system (WSTS) [2, 59] is a transition system equipped with a quasiordering $\leq \subseteq \mathrm{C} \times \mathrm{C}$ on configurations such that the following two conditions hold:

1. $\leq$ is a well-quasi-ordering $(\mathcal{W Q O})$, and

2. $\rightarrow$ is monotonic with respect to $\leq$, i.e. for all $\mathrm{c}_{1} \leq \mathrm{c}_{1}^{\prime}$ and transition $\mathrm{c}_{1} \rightarrow \mathrm{c}_{2}$, there exists a sequence $\mathrm{c}_{1}^{\prime} \stackrel{*}{\rightarrow} \mathrm{c}_{2}^{\prime}$ such that $\mathrm{c}_{2} \leq \mathrm{c}_{2}^{\prime}$.

Example 1. For a VAS, let the component-wise ordering $\leq$ be the ordering on the configurations. $\leq$ is a $\mathcal{W} \mathcal{Q O}$ and $\stackrel{\mathrm{t}}{\rightarrow}$ is monotonic with respect to $\leq$. Hence, VAS is a WSTS. We regard a configuration $c$ as a constraint whose denotation is the upward closure of $c$ with respect to $\leq$, i.e. [[c]] $=\mathrm{Up}_{\leq}(\mathrm{c})$. In this case, we say $c$ is the minimal element of $\left[[c]\right.$, that is, $c=\operatorname{Min}_{\leq}([[c]])$. Let the preorder 드 on constraints be defined as follows. $c \subseteq c^{\prime}$ iff $\operatorname{Min}_{\leq}([[c]]) \leq \operatorname{Min}_{\leq}\left(\left[\left[c^{\prime}\right]\right]\right)$. $\subseteq$ is clearly effective. It is also sound by transitivity of $\leq$, and is $\mathcal{W} \mathcal{O}$ by $\mathcal{W Q O}$ of $\leq[72]$.

It can be shown [2] that we can effectively compute, for any given $c$ and $t$, a finite set $\operatorname{next}_{\mathrm{t}}(\mathrm{c})=\left\{\mathrm{c}_{1}, \ldots, \mathrm{c}_{n}\right\}$ such that $\left\{\mathrm{c}^{\prime} \mid \mathrm{c}^{\prime} \stackrel{\mathrm{t}}{\rightarrow} \mathrm{Up}_{\leq}(\mathrm{c})\right\}=\bigcup_{i} \mathrm{Up}_{\leq}\left(\mathrm{c}_{i}\right)$. next is then effective, sound and complete with respect to the predecessor computation.

Assume a VAS with the set of initial and upward closed set of bad configurations $\mathrm{C}_{\text {init }}$ and $\mathrm{C}_{\text {bad }}$. To use the procedure "explore", we define $\varphi_{\text {start }}$ to be $\operatorname{Up}_{\leq}\left(\mathrm{C}_{b a d}\right)$, and isTarget $(\varphi)$ iff $\mathrm{C}_{\text {init }} \cap[[\varphi]] \neq \varnothing$.

By $\mathcal{W} \mathcal{Q O}$ of $\subseteq$ we conclude the reachability analysis of procedure "explore" terminates. It is also exact by soundness and completeness of next and isTarget and by soundness of $\leq$.

\subsection{Monotonic Abstraction}

There are several classes of systems that do not fit into the framework of wellstructured transition systems. The reason is that it is usually difficult to find a preorder $\leq$ that is a well-quasi-ordering for which transitions are monotonic 
(hence, ensuring exactness of the predecessor computation). A solution is to first find a $\mathcal{W Q O} \leq$ on the set of configurations and then apply monotonic abstraction $[5,4,8]$ in order to force monotonicity. For a well-quasi-ordering $\unlhd$ on configurations in $\mathrm{C}$, monotonic abstraction results in an abstract transition system $\left(\hat{\mathrm{C}}, \hat{\mathrm{C}_{\text {init }}}, \hat{\mathrm{T}}, \hat{\rightarrow}\right)$ that is an over-approximation of $\left(\mathrm{C}, \mathrm{C}_{\text {init }}, \mathrm{T}, \rightarrow\right)$ and is monotonic with respect to $\unlhd$. The intuition behind monotonic abstraction is that configuration $\mathrm{c}$ is allowed to use the outgoing transitions of any smaller configuration $c^{\prime} \unlhd c$. In fact, monotonic abstraction provides a new transition relation $\hat{\rightarrow}$ which is formally defined as follows. $\hat{\rightarrow}:=\cup_{t \in T} \stackrel{\hat{t}}{\rightarrow}$ where $\left(c_{1}, c_{3}\right) \in \stackrel{\hat{t}}{\rightarrow}$ iff $\exists \mathrm{c}_{2} \unlhd \mathrm{c}_{1}, \mathrm{c}_{2} \stackrel{\mathrm{t}}{\rightarrow} \mathrm{c}_{3}$. Clearly, $\stackrel{\mathrm{t}}{\rightarrow} \subseteq \stackrel{\hat{\mathrm{t}}}{\rightarrow}$ for each transition, thus, $\hat{\rightarrow}$ is an overapproximation of $\rightarrow$. The reachability analysis is guaranteed to terminate for the abstract transition system according to Theorem 6 because $\unlhd$ is $\mathcal{W} \mathcal{Q O}$.

Monotonic abstraction has been useful for verifying several classes of parameterized systems such as cache coherence and mutual exclusion protocols $[4,5]$.

\subsubsection{Refinement of Monotonic Abstraction}

Monotonic abstraction works well when the initial ordering can generate abstract transition systems that are safe with respect to the desired system properties, such as mutual exclusion. In such cases, it is usually enough to keep track of simple constraints on individual variables to verify the system successfully. We call such approaches plain monotonic abstraction. However, there are many classes of systems where the initial ordering suggested by plain monotonic abstraction might not be precise enough to avoid generating spurious counterexamples. In fact, plain monotonic abstraction cannot preserve the required invariants for the correctness of such systems. This often results in generating spurious counterexamples by running the reachability analysis procedure represented in Section 3.3 on the monotonically abstracted system.

Constrained Monotonic Abstraction (CMA) [3] has introduced a counterexample-guided abstraction refinement (CEGAR) [35] algorithm for iterative refinement of monotonic abstraction. The idea of CMA is to start with an initial preorder $\leq_{0}$, such as those introduced in previous works on monotonic abstraction [4] and to iteratively strengthen it.

Figure 3.1 gives an overview of CMA. The approach is based on the CEGAR framework. The inputs of the algorithm are the target transition system $\left(\mathrm{C}, \mathrm{C}_{\text {init }}, \mathrm{T}, \rightarrow\right)$ and an initial preorder $\leq_{0}$. The algorithm consists of two main modules, the reachability checker and the counterexample analyzer. In the $i$-th iteration of the procedure, the reachability checker runs backward reachability analysis on monotonically abstracted systems (procedure "explore" in Section 3.3) and tries to verify the given transition system by first abstracting it using monotonic abstraction with respect to the preorder $\leq_{i}$. If it turns out that bad configurations are reachable, a counterexample that is generated 


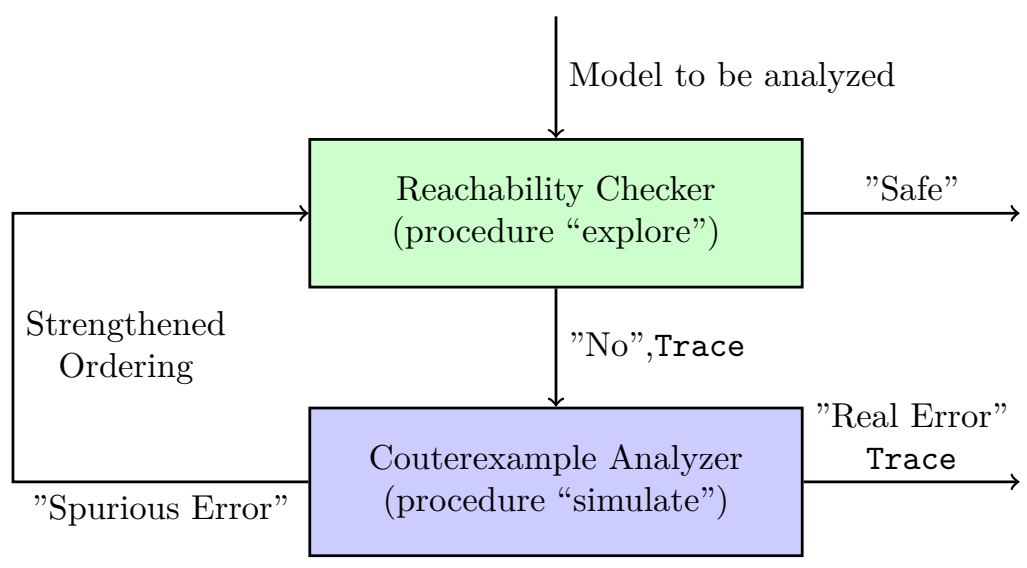

Figure 3.1: An overview of the CEGAR algorithm for refining the monotonic abstraction in CMA [3]

by the reachability analysis is fed to the counterexample analyzer represented in the procedure "simulate". The counterexample analyzer checks feasibility of the trace on the concrete transition system. It then reports either "Real Error" or "Spurious Trace".

A feasibility check is done by simulating the trace forwardly. If the counterexample is spurious, a constraint $\varphi$ is found in the $i$-th loop iteration such that none of the configurations in $\left[\left[\varphi_{j+1}\right]\right]$ is the successor of a configuration in $\llbracket\left[\varphi_{j} \rrbracket\right.$ with respect to the transition $\stackrel{\mathrm{t}_{j+1}}{\longrightarrow}$ (line 3 of the procedure "simulate"). In this case the algorithm computes the constraint $\varphi^{\prime}$ that denotes the set of predecessor configurations of $\varphi_{j+1}$ with respect to the transition $\stackrel{\mathrm{t}_{j+1}}{\longrightarrow}$ (line 4 ) and then using an interpolation technique (e.g. Craig interporlation [78, 101]) computes a constraint $\varphi_{S}$ (a so called "Safety Zone") at line 5 .

The algorithm then uses the constraint $\varphi_{S}$ to strengthen the preorder $\leq_{i}$ in the following way. A new preorder $\leq_{i+1}$ is introduced such that $\leq_{i+1} \subset \leq_{i}$ and is defined as $\leq_{i+1}:=\left\{\left(c, c^{\prime}\right) \mid c \leq_{i} c^{\prime}\right.$ and if $c^{\prime} \in\left[\left[\varphi_{S}\right]\right]$ then $c \in\left[\left[\varphi_{S}\right]\right]$. The intuition of preorder strengthening in CMA is to forbid configurations inside a "Safety Zone" to use a transition from some smaller (with respect to $\leq_{i}$ ) configuration, which is outside the "Safety Zone". The strengthened preorder helps monotonic abstraction to generate a more precise abstract transition system in the $(i+1)$-th iteration.

It can be shown by contradiction that such strengthening of the preorder preserves $\mathcal{W} \mathcal{Q O}$; hence, the backward reachability analysis in each iteration of CMA is guaranteed to terminate according to Theorem 6. This approach will be used in Chapter 6 and will be revisited and improved in Chapter 7 . 
Input: An initial constraint $\varphi_{\text {init }}$, a set of transitions $\mathrm{T}$, an interpolation function ITP, the predecessor and successor computation functions pre and post, and a counterexample $\rho=\varphi_{0} ; \mathrm{t}_{1} ; \varphi_{1} ; \ldots ; \mathrm{t}_{n} ; \varphi_{n}$

Output: "Real Error" or "Spurious Error" with a constraint $\varphi_{S}$

$1 \varphi=\varphi_{0} \wedge \varphi_{\text {init }}$

2 foreach $j=0$ to $n-1$ do

$3 \quad$ if $\left[\left[\right.\right.$ post $\left.\left._{\mathrm{t}_{j+1}}(\varphi)\right]\right]=\varnothing$ then

$4 \quad \quad \varphi^{\prime}=\operatorname{pre}_{\mathrm{t}_{j+1}}\left(\varphi_{j+1}\right)$;

$5 \quad$ return "Spurious Error", $\mathbf{I T P}\left(\varphi, \varphi^{\prime}\right)$;

$6 \quad \varphi=\operatorname{post}_{\varphi} \mathrm{t}_{j+1} \wedge \varphi_{j+1}$

7 return "Real Error";

Procedure simulate $(\rho)$, The counterexample analyzer [3] 



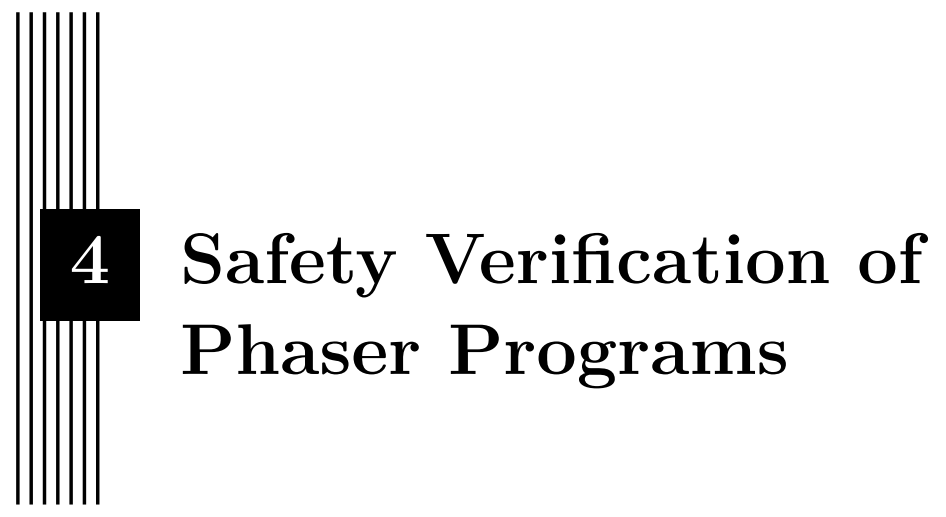

In this chapter, we focus on safety verification of programs that use phasers for synchronization $[28,29,115]$. Phasers are modern non-trivial synchronization constructs that support dynamic parallelism with runtime registration and deregistration of spawned tasks. Phasers essentially associate phases to individual tasks and use their runtime values to restrict possible concurrent executions. Unbounded phases may result in infinite transition systems even in the case of programs that create finite numbers of tasks and phasers. We define control reachability (e.g., assertion violation) and plain reachability (e.g., deadlock) and introduce an exact gap-order-based procedure that always terminates when checking control reachability for programs that generate bounded numbers of coexisting tasks and phasers. We also show that verifying plain reachability is undecidable even for a sample program that generates four tasks and three phasers. We then explain how to turn our procedure into a sound analysis for checking plain reachability. We report on experiments with our open-source prototype tool.

\subsection{Introduction}

In this chapter, we address the problem of statically checking control state reachability (as in the possibility of assertion violations, race conditions or runtime errors) and plain reachability (as in deadlock-freedom) of Phaser programs (programs that use the Phaser construct for synchronization). This sophisticated and dynamic construct unifies collective and point-to-point syn- 
chronizations. For instance, it allows for dynamic registration and deregistration of tasks. This allows for a more balanced usage of the computing resources compared to static barriers or producer-consumer constructs [114]. The construct can be added to any parallel programming language with a shared address space. For instance, it is implemented in the Habanero Java programming language, and a simplified version of it is also implemented in the java.util.concurrent library of the Java programming language. Phasers build on the clock construct from the X10 programming language [28, 29]. Phasers can be created dynamically, and the spawned tasks may get registered with or deregistered from phasers at runtime.

Intuitively, each phaser associates two phases (i.e., wait and signal phases) to each registered task. Apart from creating phasers and registering their spawned tasks with them, tasks can individually issue wait and signal commands to a phaser they are registered with. Intuitively, signal commands are used to inform other registered tasks that the issuing task is done with its signal phase. The signal command is non-blocking. It increments the signal phase associated with the issuing task on the given phaser. The wait command is instead used to check whether all registered tasks are done with (i.e., have a signal phase that is strictly larger than) the wait phase of the issuing task. This command may get blocked by a task that has not yet finished the corresponding phase. Unlike classical barriers, phasers need not force the registered tasks to wait for each other at every single phase. Instead, they allow them to proceed with the following phases (by issuing signal commands), or even to exit the construct by deregistering from it.

The possible runtime creation of tasks and phasers and their registration/deregistration enable a dynamic behavior. Moreover, the spawned tasks can work in different phases, adding flexibility to the synchronization pattern. The dynamic behavior allowed by the phaser construct suits applications that need dynamic load balancing (e.g., for solving non-uniform problems with unpredictable load estimates [128]), but comes at the price of making it easy to introduce programming mistakes such as assertion violations, race conditions, runtime registration errors and, in situations where wait and signal commands are decoupled for maximum flexibility, deadlocks. The generality of the construct also makes it interesting from a theoretical perspective, as many language constructs can be expressed using phasers. For example, synchronization barriers of Single Program Multiple Data programs, the Bulk Synchronous Parallel computation model [126], or promises and futures constructs [24] can be expressed using phasers.

We summarize our contributions presented in this chapter:

- We propose an operational model of phaser programs based on [28, 40, 115] (Section 4.4).

- We show the undecidability of checking deadlock-freedom for programs with fixed numbers of tasks and phasers (Section 4.4). 
- We describe an exact gap-order-based symbolic verification procedure for checking control state reachability (as in assertion violations, race conditions, or registration errors) and plain reachability (as in checking deadlock-freedom) in Section 4.5.

- We show termination of the procedure for control state reachability when numbers of tasks and phasers are fixed (Section 4.6).

- We describe how to turn the procedure into a sound over-approximation for plain reachability (Section 4.6).

- We report on our experiments with our open-source tool (Section 4.7).

Related work. We are not aware of automatic formal verification works other than ours (presented in Chapters 4 and 5) that focus on a dynamic synchronization construct that allows tasks to register with and deregister from the construct in runtime. Unlike [93] which proposes an approach for verifying correct synchronization of static and dynamic barriers, we focus on fully automatic verification and consider the richer and more challenging phaser construct. The work of [40] considers the dynamic verification of phaser programs and can therefore only reason about particular program inputs and runs. The work in [10] uses Java Path Finder [68] to explore several runs, but still for only one concrete input at a time. The works in $[27,100]$ target gap-order systems. Although phaser programs share some of their properties (larger gaps can do more), the results in $[27,100]$ do not apply since gap-order systems crucially forbid exact increments.

Outline. We describe a phaser program and recall some preliminaries in Sections 4.2 and 4.3. This is followed in Section 4.4 by a formal description of phaser programs and the properties we want to check. We also establish the undecidability of checking deadlock-freedom. In Section 4.5, we introduce our gap-order-based symbolic representation. In Section 4.6 we describe our verification procedure and show decidability of checking control state reachability. We then introduce our relaxation procedure for checking plain reachability. Finally, we report on our experiments and conclude the chapter.

\subsection{Illustrative Example}

The program listed in Figure 4.1 uses Boolean shared variables a, b, and done. The main task creates two phasers (lines 6 and 7 ). When creating a phaser, the task gets automatically registered with it. The main task also creates three other task instances (lines 9 to 11). Several tasks can be registered with several phasers. When a task $t$ is registered with a phaser $p$, a pair of numbers (wait $t_{p}^{t}$, sig $_{p}^{t}$ ), each in $\mathbb{N} \cup\{+\infty\}$, is associated to the couple $(t, p)$. The pair represents the individual wait and signal phases of task $t$ on phaser $p$. 


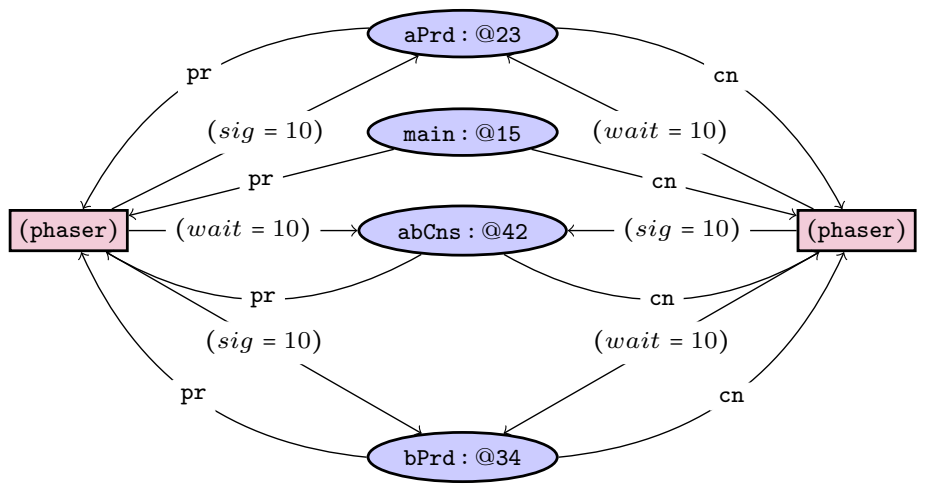

Figure 4.2: Sample wait and signal phase values for Figure 4.1. The task names are followed by their program counter. For instance, main : @15 means the task main is at line 15 of the program in Figure 4.1. Observe that there is no bound on the values of the different wait and signal phases a priori. In this example, the difference between signal and wait phases is bounded. This is not always the case in general.

mode (or in WAIT or SIG if the registrar is registered in SIG_WAIT). In this case, wait and signal phases of the newly registered task are initialized to those of the registrar. Therefore, tasks are dynamically registered (e.g., lines 9 to 11). They can also dynamically deregister themselves (e.g., line 35).

A sample configuration for the program in Figure 4.1 is illustrated by an example in Figure 4.2. In this example, two producers and one consumer are synchronized using two phasers. The consumer requires the two producers to be ahead of it (with respect to the phaser that the task main referenced with $\mathrm{pr}$ ) in order for it to consume their respective products. At the same time, the consumer needs to be ahead of both producers (with respect to the phaser main referenced with $\mathrm{cn}$ ) in order for these to produce their pair of products.

We remind the reader that phasers can be used as barriers for synchronizing dynamic subsets of concurrent tasks. Observe that producers need not, in general, proceed in a lockstep fashion. Producers may produce many items before consumers "catch up".

We are interested in checking: (a) control state reachability as in assertions (e.g., line 44), race conditions (e.g., mutual exclusion of lines 20 and 45) or runtime errors (e.g., signaling a dropped phaser), and (b) plain reachability as in deadlocks (e.g., a producer at line 22 and a consumer at line 43 with equal phases). Intuitively, both problems concern themselves with the reachability of target sets of program configurations. The difference is that control state reachability defines the targets with the states of the tasks (their control locations and whether they are registered with any phasers). Plain 
reachability can also use values or relations between values of involved phases. Observe that control state reachability depends on the values of the actual phases, but these values are not used to define the target sets. For example, assertions are expressed as predicates over Boolean variables (e.g., line 44). Establishing such an assertion requires capturing the constraints imposed by the phasers on the program behaviors.

Our work proposes a sound and complete algorithm for checking control state reachability in cases where a bounded number of tasks and phasers are generated. The algorithm can handle arbitrarily large phases, e.g., generated using nested signaling loops. The algorithm starts from a symbolic representation of all bad configurations and successively computes sets of the predecessor configurations. We show termination based on a well-quasi-ordering argument that imposes restrictions on what can be expressed with our symbolic representation. For instance, putting upper bounds on differences between phases is forbidden. Deadlock configurations cannot be faithfully captured with such restricted representations. Intuitively, a deadlocked configuration will have a cycle, where each involved task is waiting for the task to its right but where the wait phase of each task equals the signal phase of the task it is waiting for. We show the problem of checking deadlock-freedom is undecidable even for programs that only generate a bounded number of tasks and phasers. We explain how to turn our verification algorithm into a sound but incomplete procedure for checking deadlock-freedom. Precision can then be augmented on demand to eliminate false positives.

\subsection{Preliminaries}

We write $A \uplus B$ to mean the union of disjoint sets $A$ and $B$. We let $\operatorname{Pfn}(A, B)$ be the set of partial functions from $A$ to $B$ and use $\varnothing_{A}$ for the empty function over $A$, i.e., $\varnothing_{A}(a)$ is undefined (written $\left.\varnothing_{A}(a) \uparrow\right)$ for all $a \in A$. Given function $g \in \operatorname{Pfn}(A, B)$ we write $g(a) \downarrow$ to mean that $g(a)$ is defined and write $g \backslash\{a\}$ to mean the restriction of $g$ to the domain $A \backslash\{a\}$. We write $g[a \leftarrow b]$ for the function that coincides with $g$ on $A$ except for $a$ that is associated to $b$. We abuse notation and let $g\left[\left\{a_{i} \leftarrow b_{i} \mid i \in I\right\}\right]$, for a set $\left\{a_{i} \mid i \in I\right\}$ of pairwise different elements, mean the function that coincides with $g$ on $A$ except for each $a_{i}$ that is associated to the corresponding $b_{i}$. We sometimes write a function $g$ as a set $\{a \mapsto g(a) \mid a \in A\}$. It is then implicitly undefined outside of $A$.

\subsection{Phaser Programs}

We define the syntax and semantics of a core phaser program language. We make sure the simplified language presented here represents the generalpurpose languages that use phasers so that our results have a practical impact. 


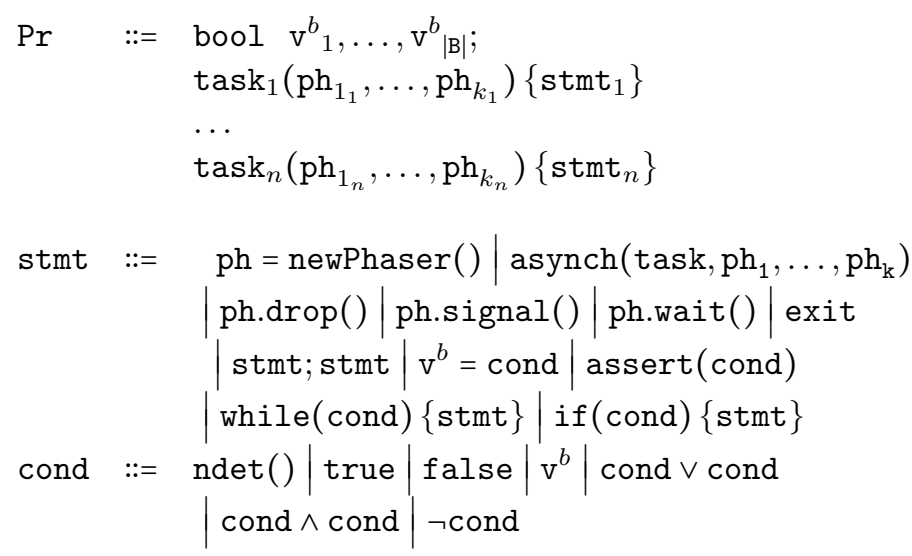

A phaser program $\operatorname{Pr}=(\mathrm{B}, \mathrm{P}, \mathrm{Ts})$ consists of a set of tasks Ts including a unique "main" task main()\{stmt\}. All task instances share a set B of Boolean variables. A task is declared with $\operatorname{task}\left(\mathrm{ph}_{1}, \ldots, \mathrm{ph}_{k}\right)\{\mathrm{stmt}\}$, where $\mathrm{ph}_{1}, \ldots \mathrm{ph}_{k}$ are phaser variables that are local to the declared task. A task can also create a new phaser with $\mathrm{ph}=$ newPhaser () and store a reference the phaser in a local variable ph. We let $\mathrm{P}$ be the union of all local phaser variables.

We write $\mathrm{s}$ to mean some control sequence in $\mathrm{S}$, and $\mathrm{hd}(\mathrm{s})$ and $\mathrm{tl}(\mathrm{s})$ to respectively mean the head and the tail of the sequence $\mathbf{s}$. We inductively define the finite set $\mathbf{S}$ of control sequences as follows. $\mathrm{S}$ is the smallest set containing: (i) suffixes of each "s" appearing in some "task $\left(\operatorname{ph}_{1_{i}}, \ldots, \operatorname{ph}_{k_{i}}\right)\{\mathbf{s}\}$ ", (ii) all $\mathbf{s}_{1}$; while(cond) $\left\{\mathbf{s}_{1}\right\}$; tr(s) if $\mathrm{s}$ is in $\mathrm{S}$ and hd(s) $=$ while(cond) $\left\{\mathrm{s}_{1}\right\}$, and (iii) all $s_{1} ; t l(s)$ if $s$ is in $S$ and $h d(s)=\operatorname{if(cond)}\left\{s_{1}\right\}$.

Example 2. Assume there is a task $\operatorname{task}(\mathrm{ph})\{\mathbf{s}\}$ in the program for which $\mathbf{s}=$ while(cond) $\{$ ph.signal(); ph.wait ()$\} ;$ ph.drop(). The set of control sequences of the program, S, will then contain while(cond) \{ph.signal(); ph.wait()\}; ph.drop(), ph.drop(), and ph.signal(); ph.wait(); while(cond) \{ph.signal(); ph.wait()\}ph.drop().

Initially, a unique task instance starts executing the main ()$\{$ stmt $\}$ task. A phaser can recall a pair of values (i.e., wait and signal) for each task that is registered with it. A task can create a new phaser with $\mathrm{ph}=$ newPhaser $($ ), get registered with it (i.e., gets zero as wait and signal values associated with the new phaser) and refer to the phaser with its local variable ph. We simplify the presentation by assuming all registrations to be in SIG_WAIT mode. Including the other modes is a matter of depriving WAIT-registered tasks of a signal value (to ensure they do not block other registered tasks) and of ensuring that issued commands respect registration modes. 
A task $\operatorname{task}\left(\mathrm{ph}_{1}, \ldots, \mathrm{ph}_{k}\right)\{$ stmt $\}$ in Ts takes the phaser variables $\mathrm{ph}_{1}, \ldots \mathrm{ph}_{k}$ as parameters (write param0f(task) to mean these parameters). A task instance can spawn another task instance with asynch(task, $\left.\mathrm{ph}_{1}, \ldots, \mathrm{ph}_{\mathrm{n}}\right)$. The issuing task instance registers the spawned task on the phasers referenced by $\mathrm{ph}_{1}, \ldots, \mathrm{ph}_{n}$, with its wait and signal values. Spawner and Spawnee execute concurrently. A task can deregister itself from a phaser referenced by a variable ph with ph.drop().

A task can issue signal or wait commands on a phaser referenced by ph and on which it is registered. A wait command on a phaser blocks until the wait value of the task instance executing the wait on the phaser is strictly smaller than the signal value of all task instances registered with the phaser. In other words, ph.wait() blocks if ph references a phaser such that at least one of the signal values stored by the phaser is equal to the wait value of the task that tries to perform the wait. A signal command does not block. It only increments the signal value of the task instance executing the signal command on the phaser.

Note that assignment of phaser variables is excluded from the syntax; additionally, we restrict task creation asynch(task, $\left.\mathrm{ph}_{1}, \ldots, \mathrm{ph}_{\mathrm{n}}\right)$ and require that parameter variables $\mathrm{ph}_{i}$ are all different. This prevents two variables from reference the same phaser and avoids the need to deal with aliasing: we can reason on the single variable in a process that references a phaser. Extending our work to deal with aliasing is easy but would require heavier notations.

We will need the notions of configurations, partial configurations, and inclusion in order to define the reachability problems we consider in this chapter. We introduce them in the following and assume a phaser program $\operatorname{Pr}=(\mathrm{B}, \mathrm{P}, \mathrm{Ts})$.

Configurations. Configurations of a phaser program describe valuations of its variables, control sequences of its tasks, and registration details of the tasks with the phasers.

A configuration $c$ of $\operatorname{Pr}=(\mathrm{B}, \mathrm{P}, \mathrm{Ts})$ is a tuple $(\mathcal{T}, \mathcal{P}, 6 v, p c, p v, \phi)$, where:

- $\mathcal{T}$ is the current finite set of task identifiers. Let $t, u$ range over $\mathcal{T}$,

- $\mathcal{P}$ is the current finite set of phaser identifiers. Let $p, q$ range over $\mathcal{P}$,

- $6 v: \mathrm{B} \rightarrow\{$ true, false $\}$ is a total mapping that associates a value to each Boolean variable $\mathrm{v}^{b}$ in the set $\mathrm{B}$.

- $p c: \mathcal{T} \rightarrow \mathrm{S}$ is a total mapping that associates tasks to their remaining sequences (i.e., control location),

- $p v: \mathcal{T} \rightarrow \operatorname{Pfn}(\mathrm{P}, \mathcal{P})$ is a total mapping that associates, to each task identifier in $\mathcal{T}$, a partial mapping from the local phaser variables $\mathrm{P}$ to phaser identifiers $\mathcal{P}$. It captures the values of the phaser variables $\mathrm{P}$ of each task, 
- $\phi: \mathcal{P} \rightarrow \operatorname{Pfn}\left(\mathcal{T}, \mathbb{N}^{2}\right)$ is a total mapping that associates to each phaser $p \in \mathcal{P}$ a partial mapping $\phi(p)$ that is defined exactly on the identifiers of the tasks registered with $p$. For such a task $t, \phi(p)(t)$ is the pair $\left(w_{a i t}^{t}, s i g_{p}^{t}\right)$ representing wait and signal values of $t$ on $p$.

\subsubsection{Semantics of Phaser Programs}

Given a program $\mathrm{Pr}=(\mathrm{B}, \mathrm{P}, \mathrm{Ts})$, the main task main ()$\{$ stmt $\}$ starts executing its stmt from an initial configuration $\mathrm{c}_{\text {init }}=$ $\left(\left\{t_{0}\right\},\{\}, b v_{\text {false }},\left\{t_{0} \mapsto\right.\right.$ stmt $\left.\}, \varnothing, \varnothing\right)$, where a "main" task with identifier $t_{0}$ and code stmt is the unique initial task. No phasers are present in the initial configuration, and all shared Boolean variables are mapped to false. $\phi$ is the empty function with an empty domain $\varnothing_{\varnothing}$. After a task $t$ executes a ph := newPhaser () statement (rule newPhaser in Figure 4.3), a new phaser $p$ is associated to the variable ph using $p v$ and $\phi(p)$ becomes the partial function $\{t \mapsto(0,0)\}$.

Given two configurations $c$ and $c^{\prime}$ with $c=(\mathcal{T}, \mathcal{P}, 6 v, p c, p v, \phi)$, we write $\mathrm{c} \underset{t}{\rightarrow} \mathrm{c}^{\prime}$ if there is a task $t \in \mathcal{T}$ such that one of the rules in Figure 4.3 holds. We use $\stackrel{*}{\rightarrow}$ for the reflexive transitive closure of $\rightarrow$ and write $\mathrm{c} \stackrel{*}{\rightarrow} \mathrm{c}^{\prime}$ to mean that $c^{\prime}$ is reachable from $c$. A configuration is said to be reachable if it is reachable from the initial configuration $c_{\text {init }}$. We explain in the following the operational semantic rules of the phaser programs listed in Figure 4.3.

- Rule asynch. This rule is one of the two rules that alter the set of tasks $\mathcal{T}$. Using asynch(task, $\mathrm{ph}_{1}, \ldots, \mathrm{ph}_{\mathrm{n}}$ ) statement, a task $t$ spawns a new task $u$ which is not already in the set of tasks $\mathcal{T}$. $t$ is said to be the parent of $u$. According to the definition of the task, $k$ distinct phasers in $\mathcal{P}$ need to be passed to $u$. The wait and signal phases of $t$ will be assigned as phases of $u$ for all of the phasers passed to it. More formally, for each passed phaser $p_{i}, \phi\left(p_{i}\right)(t)$ will be set to $\phi\left(p_{i}\right)(u)$. Moreover, $u$ will start execution from $s_{1}$ which is the first statement in the body of task.

- Rule exit. This rule is the other rule that alters the set of tasks $\mathcal{T}$. A task $t$ can run the exit statement to remove itself from the program. Essentially, this boils down to removing the task $t$ from $\mathcal{T}$, removing its remaining control sequence from $p c$, and removing its signal and wait phases from $\phi$.

- Rule newPhaser. The set of phasers $\mathcal{P}$ is updated upon the creation of new phasers. When a new phaser $p$ is created by task $t, p$ is added to the set of phasers $P, t$ is registered with $p$ with zero phases as its wait and signal phases. 


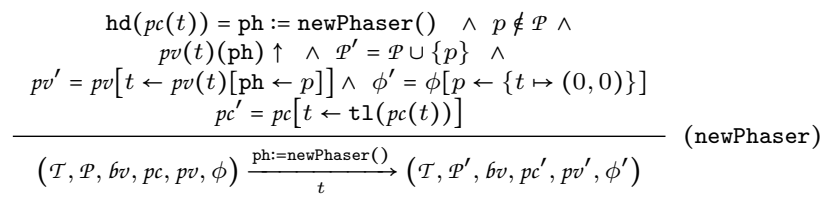

$\operatorname{hd}(p c(t))=\operatorname{asynch}\left(\right.$ task $\left., \mathrm{ph}_{1}, \ldots \mathrm{ph}_{\mathrm{k}}\right)\left\{\mathbf{s}_{1}\right\} \wedge \operatorname{param0f}($ task $)=\left(\mathrm{q}_{1}, \ldots \mathrm{q}_{k}\right) \wedge$ for each $i: 1 \leq i \leq k . p v(t)\left(\mathrm{ph}_{i}\right)=p_{i} \wedge \phi\left(p_{i}\right) \downarrow \wedge$

$u \notin \mathcal{T} \wedge p v^{\prime}=p v\left[u \leftarrow\left\{\mathrm{q}_{i} \mapsto p_{i} \mid 1 \leq i \leq k\right\}\right] \wedge p c^{\prime}=p c\left[u \leftarrow \mathrm{s}_{1}\right] \wedge$ $\phi^{\prime}=\phi\left[\left\{p_{i} \leftarrow \phi\left(p_{i}\right)\left[u \leftarrow \phi\left(p_{i}\right)(t)\right] \mid 1 \leq i \leq k\right\}\right]$

$\overline{(\mathcal{T}, \mathcal{P}, 6 v, p c, p v, \phi) \underset{\mathrm{asynch}\left(\mathrm{task}, \mathrm{ph}_{1}, \ldots \mathrm{ph}_{\mathrm{k}}\right)\left\{\mathrm{s}_{1}\right\}}{t}\left(\mathcal{T} \cup\{u\}, \mathcal{P}, 6 v, p c^{\prime}[t \leftarrow \mathrm{tl}(p c(t))], p v^{\prime}, \phi^{\prime}\right)}$

$\operatorname{hd}(p c(t))=$ ph.signal ()$\wedge p v(t)(\mathrm{ph})=p \wedge \phi(p)(t)=\left(\right.$ wait $\left._{p}^{t}, \operatorname{sig}_{p}^{t}\right) \wedge$ $\phi^{\prime}=\phi\left[p \leftarrow \phi(p)\left[t \leftarrow\left(w_{a i t}^{t}, 1+s i g_{p}^{t}\right)\right]\right]$

$(\mathcal{T}, \mathcal{P}, b v, p c, p v, \phi) \stackrel{\text { ph.signal }()}{\longrightarrow}\left(\mathcal{T}, \mathcal{P}, 6 v, p c[t \leftarrow \mathrm{tl}(p c(t))], p v, \phi^{\prime}\right)$

$\operatorname{hd}(p c(t))=\operatorname{ph} . w a i t() \wedge p v(t)(\mathrm{ph})=p \wedge \phi(p)(t)=\left(w_{a i t}^{t}, s i g_{p}^{t}\right) \wedge$

$\forall u \in \mathcal{T} .\left(\phi(p)(u)=\left(\right.\right.$ wait $_{p}^{u}$, sig $\left._{p}^{u}\right) \Rightarrow$ wait $_{p}^{t}<$ sig $\left._{p}^{u}\right) \wedge$ $\phi^{\prime}=\phi\left[p \leftarrow \phi(p)\left[t \leftarrow\left(1+w_{a i t}^{t}\right.\right.\right.$, sig $\left.\left.\left._{p}^{t}\right)\right]\right]$

$(\mathcal{T}, P, b v, p c, p v, \phi) \stackrel{\text { ph.wait }()}{t}\left(\mathcal{T}, \mathcal{P}, 6 v, p c[t \leftarrow \mathrm{tl}(p c(t))], p v, \phi^{\prime}\right)$

$\operatorname{hd}(p c(t))=\mathrm{ph} \cdot \operatorname{drop}() \wedge p v(t)(\mathrm{ph})=p \wedge \phi(p)(t) \downarrow \wedge \phi^{\prime}=\phi[p \leftarrow \phi(p)[t \leftarrow \uparrow]]$

$(\mathcal{T}, P, 6 v, p c, p v, \phi) \stackrel{\text { ph.drop }()}{\longrightarrow}\left(\mathcal{T}, P, 6 v, p c[t \leftarrow \mathrm{tl}(p c(t))], p v, \phi^{\prime}\right)$

$\operatorname{hd}(p c(t))=\operatorname{exit} \wedge p v^{\prime}=p v \backslash\{t\} \wedge p c^{\prime}=p c \backslash\{t\} \wedge$ $\phi^{\prime}=\phi[\{p \leftarrow(\phi(p) \backslash\{t\}) \mid p \in \mathcal{P}\}]$

$(\mathcal{T}, \mathcal{P}, 6 v, p c, p v, \phi) \underset{t}{\stackrel{\text { exit }}{\longrightarrow}}\left(\mathcal{T} \backslash\{t\}, \mathcal{P}, 6 v, p c^{\prime}, p v^{\prime}, \phi^{\prime}\right)$ (exit)

$\frac{\operatorname{hd}(p c(t))=\operatorname{assert}(\text { cond }) \wedge b v(\text { cond })=\text { true }}{(\mathcal{T}, \mathcal{P}, 6 v, p c, p v, \phi) \stackrel{\text { assert }(\text { cond })}{t}(\mathcal{T}, \mathcal{P}, b v, p c[t \leftarrow \operatorname{tl}(p c(t))], p v, \phi)}$ (assert.ok)

$\frac{\operatorname{hd}(p c(t))=\operatorname{assert}(\text { cond }) \wedge b v(\text { cond })=\text { false }}{(\mathcal{T}, \mathcal{P}, b v, p c, p v, \phi) \in \text { badConf } \mathbf{s}_{\text {assert }}^{(|\mathcal{T}|,|\mathcal{T}|)}}\left(\begin{array}{c}\text { assert. } \\ \text { fault }\end{array}\right)$

$\operatorname{hd}(p c(t))=\mathrm{s} \wedge(\mathrm{s}=$ ph.drop ()$\vee \mathrm{s}=$ ph.signal ()

$\vee \mathrm{s}=\operatorname{ph}$. wait ()$\vee \mathrm{s}=\operatorname{asynch}(\operatorname{task}, \ldots, \operatorname{ph}, \ldots)) \wedge$

$\frac{(p v(t)(\mathrm{ph}) \uparrow \vee \phi(p v(t)(\mathrm{ph}))(t) \uparrow)}{(\mathcal{T}, \mathcal{P}, b v, p c, p v, \phi) \in \text { badConf } \mathbf{s}_{\text {register }}^{(|\mathcal{T}|,|\mathcal{T}|)}} \quad\left(\begin{array}{c}\begin{array}{c}\text { registration } \\ \text { error }\end{array}\end{array}\right)$

$\left\{t_{0}, \ldots t_{n-1}\right\} \subseteq \mathcal{T} \wedge\left\{p_{0}, \ldots p_{n-1}\right\} \subseteq \mathcal{P} \wedge \forall i: 0 \leq i \leq(n-1) \cdot \operatorname{hd}\left(p c\left(t_{i}\right)\right)=\operatorname{ph}_{i} \cdot \operatorname{wait}() \wedge$ $p v\left(t_{(i+1) \%_{n}}\right)\left(\mathrm{q}_{i}\right)=p_{i} \wedge p v\left(t_{i}\right)\left(\mathrm{ph}_{i}\right)=p_{i} \wedge$ wait $_{p_{i}}^{t_{i}}=\operatorname{sig}_{p_{i}}^{t}(i+1) \% n$

$(\mathcal{T}, \mathcal{P}, 6 v, p c, p v, \phi) \in$ badConf $\mathbf{s}_{\text {deadlock }}^{(|| \mathcal{|}|,| q \mid)}$

$\operatorname{hd}(p c(t))=\mathrm{v}^{b}:=$ cond $\wedge \operatorname{hd}(p c(u))=\mathrm{s}^{\prime} \wedge t \neq u \wedge$

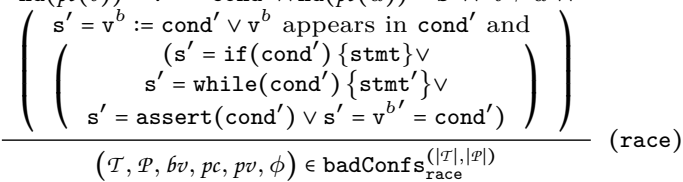

Figure 4.3: Operational semantics of phaser statements. 
- Rule signal. The ph.signal() statement increments the signal value of the issuing task $t$ on phaser $p$, i.e. $s i g_{p}^{t}$, by one.

- Rule wait. The ph.wait() statement similarly increments the wait phase of the issuing task, i.e. wait ${ }_{p}^{t}$, given that for all other tasks $u$ registered with $p$ the condition $s i g_{p}^{t}<s i g_{p}^{u}$ holds. Otherwise, $t$ blocks until the condition holds.

- Rule drop. A task $t$ can deregister itself from a phaser $p$ using the ph.drop() statement. This will essentially remove the phaser variable ph assigned to $p$ and also its wait and signal values at $p$, i.e. $\phi(p)(t)$. asynch and drop statements allow dynamic registration with and deregistration from phasers, hence, a lot of freedom in synchronization patterns in phaser programs.

- Rule registration error. The mapping pv associates values to program phaser variables. Accessing variables with undefined values, or phasers to which $t$ is not currently registered leads to registration errors. This rule is used to characterize the set badConf $\mathrm{s}_{\text {register }}^{(n, m)}$ of configurations that lead to registration errors.

- Rules assert. fault and assert. ok. An assert(cond) statement would result in an error if its condition cond does not hold. Rule assert. fault formally defines the configurations in the set badConf $\mathbf{s}_{\text {assert }}^{(n, m)}$. On the other hand, if the condition cond holds, only the control sequence of the executing task $t$ will be updated according to rule assert. ok.

- Rule deadlock characterizes the deadlocked configurations in badConf $\mathbf{s}_{\text {deadlock }}^{(n, m)}$ and is explained in Definition 3 and Definition 4 below.

Rule race formally defines configurations with race conditions, i.e. those in the set badConf $\mathbf{s}_{\text {race }}^{(n, m)}$. A race condition occurs if distinct tasks $t$ and $u$ are about to read and write to the shared variable $\mathrm{v}^{b}$ at the same time.

\section{Control-state Reachability}

Checking the possibility of assertion violations, of registration errors and of race conditions amounts to checking reachability of configurations respectively in badConf $\mathbf{s}_{\text {assert }}^{(n, m)}$, badConf $\mathbf{s}_{\text {register }}^{(n, m)}$ and in badConf $\mathbf{s}_{\text {race }}^{(n, m)}$ for some number of tasks $n$ and number of phasers $m$. In Chapter 4.5, we introduce a complete procedure for checking reachability of such sets of configurations and show it to be sound for programs with fixed upper bounds on numbers of generated phasers and tasks. 


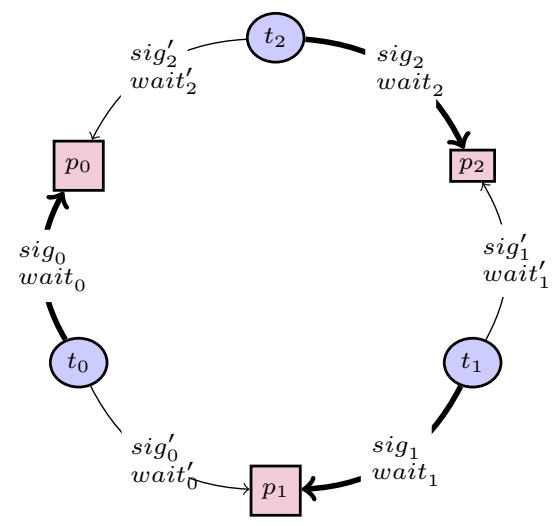

Figure 4.4: A sample deadlock configuration with three tasks and three phasers

\section{Plain Reachability and Deadlocks}

Besides control state reachability, we are also interested in checking for the possibility of deadlocks. For this, we need to define the notion of a blocked task. Assume in the following a configuration $c=(\mathcal{T}, \mathcal{P}, 6 v, p c, p v, \phi)$.

Definition 3 (Blocked). A task $t \in \mathcal{T}$ is blocked at phaser $p \in \mathcal{P}$ by task $u \in \mathcal{T}$ if $\mathrm{hd}(p c(\mathrm{t}))=$ ph.wait () with $p v(t)(\mathrm{ph})=p$ and $\phi(p)(t)=\left(\right.$ wait $\left._{p}^{t},{ }_{-}\right)$when $\phi(p)(u)=\left(\ldots, s i g_{p}^{u}\right)$ and sig $_{p}^{u} \leq$ wait $_{p}^{t}$.

Intuitively, a task $t$ is blocked by a task $u$ if it cannot finish its wait command on some phaser because it is waiting for task $u$ that have not issued enough signal commands on the same phaser.

Definition 4 (Deadlock). $(\mathcal{T}, \mathcal{P}, 6 v, p c, p v, \phi)$ is a deadlock configuration if each task of a none-empty subset $\mathcal{U} \subseteq \mathcal{T}$ is blocked by some task in $\mathcal{U}$.

Example 3. In Figure 4.4, a sample deadlock configuration with three tasks and three phasers is illustrated. For each $i: 0 \leq i \leq 2$, task $t_{i}$ is blocked on the

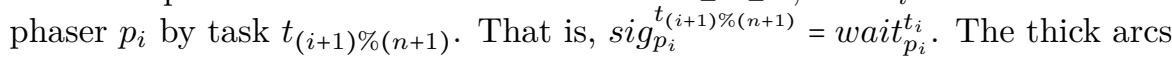
represent the blockage of a task on a phaser.

Theorem 7 (Deadlock-Freedom). It is undecidable in general, even for programs with only three phasers and four tasks, to check for deadlock-freedom.

Proof. We encode the reachability problem of any given 3-counter reset-VAS (Section 3.2.3) as the reachability problem of a configuration with a simple cycle involving three tasks. Indeed, reachability of configuration $\left(\mathrm{q}_{\mathrm{F}}, 0,0,0\right)$ (three counters $x_{1}, x_{2}, x_{3}$ with zero values at some control location $\mathrm{q}_{\mathrm{F}}$ ) is 


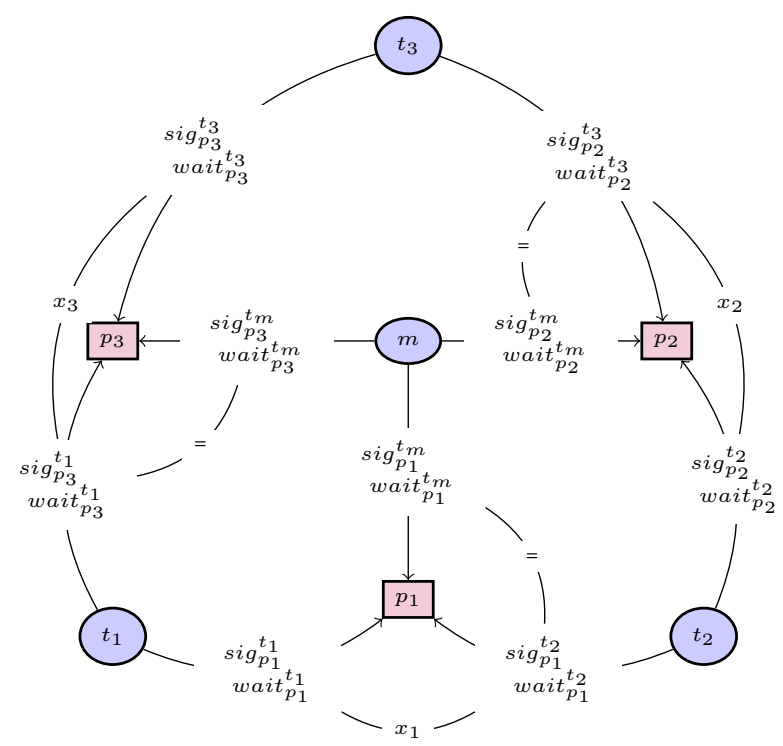

Figure 4.7: A deadlock configuration generated by proof of the Theorem 7

We explain in the following how we encode the increment, decrement, and reset operations on counter $x_{1}$ in a way that its invariant is preserved. The counters $x_{2}$ and $x_{3}$ are similar.

Increment. To model incrementing counter $x_{1}$, main sets $\mathrm{t} 1:$ inc $: \mathrm{x} 1$ to true (line 31) and waits for ok to be true (line 32). Because of the message t1 : inc: $\mathrm{x} 1$, only $t_{1}$ can proceed. It issues a signal on ph1 at line 4 of its procedure in Figure 4.6. This operation does not involve any variable that is present in the invariant; hence, the invariant is preserved after the increment.

Decrement. To model decrementing counter $x_{1}$, main first issues a ph1.signal(), then sets $\mathrm{t} 2: \operatorname{dec}: \mathrm{x} 1$ to true at line 40 of Figure 4.5 , and finally, waits for ok to hold. Because of the message $\mathrm{t} 2: \operatorname{dec}: \mathrm{x} 1$, only $t_{2}$ can participate in decrementing $x_{1}$. It does so by issuing a ph1.signal() and ph1.wait() at lines 8 and 9 of its procedure in Figure 4.6. Finally, main performs ph1.wait() at line 42 of Figure 4.5. Hence, the invariant

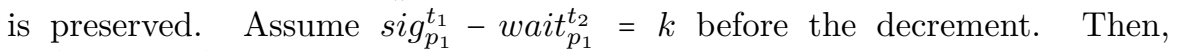
$\operatorname{sig}_{p_{1}}^{t_{1}}-\left(\right.$ wait $\left._{p_{1}}^{t_{2}}+1\right)=k-1$ after the decrement. We are sure that ph1.wait() is enabled since by the semantics of counter machines, $x_{1}$ can only be decremented if $x_{1}>0$ and we have shown that for every increment of $x_{1}, s i g_{p_{1}}^{t_{1}}$ is incremented by one. Moreover, $\mathrm{ph}_{1}$ is only registered with main, tsk1 and tsk2 and we have shown that all of them have already issued ph1.signal(). 
Reset. To reset counter $x_{1}$, main first issues a ph3.signal() and then sets t1 : res : $\mathrm{x} 1$ to true (line 50 of Figure 4.5) and waits for ok to hold. According to $\mathrm{t} 1 \mathrm{inc}: \mathrm{x} 1, t_{1}$ will proceed by setting $\mathrm{t} 3: \mathrm{res}: \mathrm{x} 1$ to true at line 13 of procedure tsk1 in Figure 4.6. It then exits at line 14. Now, tsk3 will issue ph3.signal() and ph3.wait() and set ok to true at lines 17 to 19 of its procedure. Thereafter, main performs ph3.wait() at line 53 of Figure 4.5. Intuitively, main does so to wait for $t_{1}$ to exit. Note that $t_{1}$ and $t_{3}$ are the only tasks other than main that are registered with ph3. The operations on lines 49 and 53 of main together with those of the lines 17 and 18 of tsk3 ensure that the invariant is preserved for ph3.

Finally, main spawns a new tsk1 and passes the phasers ph1 and ph3 to it. By the semantics of phaser programs, tsk1 will now copy the phases of main on ph1 and ph3 which are respectively equal to the phases of tsk2 and old values of tsk1 on those phasers (according to the invariant). Hence, $s i g_{p_{1}}^{t_{1}}-w_{a i t} t_{p_{1}}^{t_{2}}=0$. Moreover, $s g_{p_{3}}^{t_{3}}-w_{a i t} t_{p_{3}}^{t_{1}}$ will remain intact.

Finally, asking each task $t_{i}$ to perform a wait on phaser $p_{(i+1) \% 3+1}$ ensures that a simple cycle of size 3 is built exactly when the three counters are 0 according to the invariant.

We have just demonstrated undecidability for checking deadlock in phaser programs. In the next section, we introduce the symbolic representation that will be used in our verification procedure.

\subsection{Symbolic Verification of Phaser Programs}

We briefly introduce gap-order constraints and use them to define a symbolic representation (hereafter constraints) that we use in Chapter 4.6 for checking reachability.

\subsubsection{Gap-order Constraints and Graphs $[113,91,27,100]$}

Gap-order constraints can be regarded as a particular case of octagons or unit two variables per inequality (utvpi) constraints. Assume in this section that $x$ and $y$ are integer variables and that $k$ is an integer constant. We use $X$ and $Y$ to mean finite sets of integer variables. A valuation val is a total function $X \rightarrow \mathbb{Z}$. Valuations are implicitly extended to preserve constants (i.e., $\operatorname{val}(k)=k$ for any $k \in \mathbb{Z})$.

A gap-order clause $\delta$ over $X$ is an inequality of the form $a-b \geq k$, where $a, b \in X \cup\{0\}$. A gap-order constraint $\Delta$ over $X$ is a finite conjunction of gap-order clauses over the same set $X$. Observe that $(x=y+2 \wedge y \leq 5)$ is essentially a gap-order constraint because it can be equivalently rewritten as the conjunction $(x-y \geq 2 \wedge y-x \geq-2 \wedge 0-y \geq-5)$. 
Given a gap-order constraint $\Delta$ over $X$ and a valuation val $: X \rightarrow \mathbb{Z}$, we write val $\vDash \Delta$ to mean that $\operatorname{val}(a)-\operatorname{val}(b) \geq k$ holds for each gap-order clause $\delta: a-b \geq k$ appearing in $\Delta$. We let $\operatorname{Sat}(\Delta)$ be the set $\{\operatorname{val}: X \rightarrow \mathbb{Z} \mid \operatorname{val} \vDash \Delta\}$.

A gap-order graph (or graph for short) $\wp$ over $X$ is a graph $(V, E)$ with vertices $V=X \cup\{0\}$, where edges in $E$ are of the form $a \stackrel{k}{\rightarrow} b$ with $a, b \in V$ and weight $k$ in $\mathbb{Z} \cup\{-\infty,+\infty\}$. We let varsOf $(\wp)=X$.

Given a gap-constraint $\Delta$ over $X$, we can build the graph graph0f $(\Delta)$ with vertices $X \cup\{0\}$ and where $E$ only contains a representative $a \stackrel{k}{\rightarrow} b$ edge for each clause $a-b \geq k$ appearing in $\Delta$.

A valuation val $: X \rightarrow \mathbb{Z}$ satisfies a graph $\wp=(V, E)$ (written val $\vDash$ $\wp)$ iff $\operatorname{val}(a)-\operatorname{val}(b) \geq k$ for each $a \stackrel{k}{\rightarrow} b \in E$. We let $\operatorname{Sat}(\wp)$ be the set $\{$ val $: X \rightarrow \mathbb{Z} \mid$ val $\vDash \wp\}$. Clearly, $\operatorname{Sat}(\operatorname{graph0f}(\Delta))=\operatorname{Sat}(\Delta)$.

The closure clo $(\wp)$ of a graph $\wp=(V, E)$ is the unique complete graph with the same vertices $V$ and where $a \stackrel{k^{\prime}}{\rightarrow} b$ is an edge of clo $(\wp)$ iff $k^{\prime} \epsilon$ $\mathbb{Z} \cup\{-\infty,+\infty\}$ is the least upper bound of all weight-sums for any path in $\wp$ from $a$ to $b$. Closure allows us to deduce $(0-x \geq-7)$ from $(y-x \geq-2 \wedge$ $0-y \geq-5)$. The result of the closure procedure is a unique graph $\wp_{\text {false }}$ denoting the graph without any satisfying valuation each time a weight $\mathrm{k}=+\infty$ is generated. The closure of a graph can be computed in polynomial time and we get $\operatorname{Sat}(\mathrm{clo}(\wp))=\operatorname{Sat}(\wp)$.

We define the degree of a graph $\wp$ (written degreeOf $(\wp))$ to be 0 if no edge in clo $(\wp)$ has a negative weight apart from $-\infty$. Otherwise, degree $0 f(\wp)$ is the largest natural number $k \in \mathbb{N}$ such that there is an edge in clo $(\wp)$ with weight $-k$. For instance, the degree of the graph resulting from $(x-y \geq$ $2 \wedge y-x \geq-4)$ is 4 . We systematically close all manipulated graphs and write $\mathcal{G}(X)$ for the set of closed graphs over $X$.

Given a graph $\wp$, we write $\wp[y / x]$ to mean the graph obtained by replacing the vertex $x$ with the vertex $y$. We abuse notation and write $\wp\left[\left\{y_{i} / x_{i} \mid i \in I\right\}\right]$, for pairwise different $x_{i}$ elements to mean the simultaneous application of the individual substitutions.

For a set of variables $Y$, we write $\wp \ominus Y$ to mean the graph obtained by removing the variables in $Y$ from the vertices of $\wp$. Given two closed graphs $\wp$ and $\wp^{\prime}$ over the same $X$, we write $\wp \leq_{\mathcal{G}} \wp^{\prime}$ to mean that each directed edge in $\wp$ is labeled with a larger or equal weight in $\wp^{\prime}$. As a result, $\operatorname{Sat}\left(\wp^{\prime}\right) \subseteq \operatorname{Sat}(\wp)$. Finally, we write $\wp \otimes \wp^{\prime}$ to mean the closure of the graph obtained by merging the two sets of vertices and edges. As a result, $\operatorname{Sat}\left(\wp \otimes \wp^{\prime}\right)=\operatorname{Sat}(\wp) \cap \operatorname{Sat}\left(\wp^{\prime}\right)$.

\subsubsection{Symbolic Representation}

A constraint $\varphi$ is a tuple $(\mathcal{T}, \mathcal{P}, 6 v, p c, p v, \gamma)$, where the only difference with the definition of a configuration $(\mathcal{T}, \mathcal{P}, b v, p c, p v, \phi)$ is the adoption of a gap-order constraint $\gamma$ instead of $\phi$. More specifically, $\gamma: \mathcal{P} \rightarrow \cup_{\mathcal{U \subseteq T} \mathcal{T}} \mathcal{G}\left(\cup_{t \in \mathcal{U}}\left\{\omega^{t}, \sigma^{t}\right\}\right)$ is a total mapping that associates a gap-order graph to each phaser $p \in \mathcal{P}$. 
Intuitively, we use the variables $\omega^{t}$ and $\sigma^{t}$ to constrain in graph $\gamma(p)$ possible values of wait $\left(w a i t_{p}^{t}\right)$ and signal $\left(s i g_{p}^{t}\right)$ phases of each task $t$ registered with phaser $p$. As a result, we can check if task $t$ is registered with phaser $p$ according to graph $\wp=\gamma(p)$ by checking if $\left\{\omega^{t}, \sigma^{t}\right\} \subseteq \operatorname{varsOf}(\wp)$. We will write $\operatorname{Reg}(p, \wp)$ to mean the set of tasks $\left\{t \mid\left\{\omega^{t}, \sigma^{t}\right\} \subseteq \operatorname{vars0f}(\wp)\right\}$. We also write isReg $(t, p, \wp)$ for the predicate $t \in \operatorname{Reg}(p, \wp)$.

Observe that the language semantics impose that, for each phaser $p$ and for any pair $t, u$ of tasks in $\operatorname{Reg}(p, \wp)$, the predicate $0 \leq w_{a i t}^{t} \leq s i g_{p}^{u}$ is an invariant. For this reason, we always safely strengthen, in any obtained $\gamma(p)=\wp$, weights $k$ in $\sigma^{t} \stackrel{k}{\rightarrow} \omega^{u}, \sigma^{t} \stackrel{k}{\rightarrow} 0$ and $\omega^{t} \stackrel{k}{\rightarrow} 0$ with $\max (k, 0)$. The following definition helps us characterize constraints for which our procedure terminates.

Definition 5 (Degree and freeness of constraints). The degree of a constraint $(\mathcal{T}, \mathcal{P}, 6 v, p c, p v, \gamma)$ is 0 if $\mathcal{P}$ is empty. Otherwise, its degree is the greatest degree among all its graphs $\gamma(p)$ for $p \in \mathcal{P}$. Furthermore, a constraint is said to be "free" if, for any $p \in \mathcal{P}$, the only edges in $\gamma(p)$ with weights different from $-\infty$ are edges of the forms (i) $\sigma^{t} \stackrel{k_{\left(\sigma^{t}, \omega^{u}\right)}}{\longrightarrow} \omega^{u}$, (ii) $\sigma^{t} \stackrel{k_{\left(\sigma^{t}\right)}}{\longrightarrow} 0$, or (iii) $\omega^{t} \stackrel{k_{\left(\omega^{t}\right)}}{\longrightarrow} 0$ for some $t, u \in \operatorname{Reg}(p, \gamma(p))$ and $k_{\left(\sigma^{t}, \omega^{u}\right)}, k_{\left(\sigma^{t}\right)}, k_{\left(\omega^{t}\right)} \in \mathbb{N}$

Free constraints are only allowed to impose, for the same phaser, nonnegative lower bounds on differences between signals and waits, between signals and 0 , and between waits and 0 . Like degree-0-constraints, free constraints are not allowed to put a positive upper bound on how much larger a signal is than a wait. Unlike degree-0-constraints, they cannot put bounds on the differences among signal values or wait values. For instance, a free constraint cannot impose $\sigma^{t}-\sigma^{u}=0$ while a degree-0-constraint can. Intuitively, freeness does not oblige our verification procedure to maintain exact differences when firing signal or wait instructions, jeopardizing termination. This will be stated in Section 4.6.

\subsubsection{Denotations of Constraints}

Given a configuration $\mathrm{c}=(\mathcal{T}, \mathcal{P}, 6 v, p c, p v, \phi)$ and a constraint $\varphi=$ $\left(\mathcal{T}^{\prime}, \mathcal{P}^{\prime}, 6 v^{\prime}, p c^{\prime}, p v^{\prime}, \gamma^{\prime}\right)$, we say that c satisfies $\varphi$, and write $\mathrm{c} \vDash \varphi$, if c satisfies (up to a renaming of the tasks and the phasers) the conditions imposed by $\varphi$. More concretely, $\mathrm{c} \vDash \varphi$ if $6 v=6 v^{\prime}$ and there are bijections $\kappa: \mathcal{T} \rightarrow \mathcal{T}^{\prime}$ and $\pi: \mathscr{P} \rightarrow \mathcal{P}^{\prime}$ such that:

- $p c(t)=p c^{\prime}(\kappa(t))$ for each $t \in \mathcal{T}$;

- $\pi(p v(t)(\mathrm{ph}))=p v^{\prime}(\kappa(t))(\mathrm{ph})$ for each $t \in \mathcal{T}$ and $\mathrm{ph} \in \mathrm{P}$

- the renaming of tasks and phasers in $\phi$ with respect to $\kappa$ and $\pi$ satisfies $\gamma$, i.e., 
- for each $t \in \mathcal{T}$ and each $p \in \mathcal{P}$, isReg $(\kappa(t), \pi(p), \gamma(\pi(p)))$ iff $\phi(p)(t) \downarrow$,

- for each $p^{\prime} \in \mathcal{P}^{\prime}, \operatorname{val}_{p^{\prime}} \vDash \gamma\left(p^{\prime}\right)$, where $\operatorname{val}_{p^{\prime}}$ is the valuation that for each $t^{\prime} \in \mathcal{T}^{\prime}$ associates $\omega^{t^{\prime}}$ to wait $_{p^{\prime}}^{t^{\prime}}$ and $\sigma^{t^{\prime}}$ to $s i g_{p^{\prime}}^{t^{\prime}}$ if $\left.\left.\phi\left(\pi^{-1}\left(p^{\prime}\right)\right)\left(\kappa^{-1}\left(t^{\prime}\right)\right)\right)\right)=\left(w_{a i t} t_{p^{\prime}}^{t^{\prime}}, s_{i} g_{p^{\prime}}^{t^{\prime}}\right)$. Note that $\gamma\left(p^{\prime}\right)$ is by definition a graph over variables $\left(\omega^{t^{\prime}}, \sigma^{t^{\prime}}\right)$.

Intuitively, $\left[\left(\mathcal{T}^{\prime}, \mathcal{P}^{\prime}, 6 v^{\prime}, p c^{\prime}, p v^{\prime}, \gamma^{\prime}\right)\right]$ contains all configurations c with the same number of tasks and phasers and such that there are renamings of tasks and phasers that preserve in $c$ the correspondence between $p c, p v$ and $\gamma$. We write $\left[[\Phi]\right.$, for a set $\Phi$ of constraints, to mean the union $\cup_{\varphi \in \Phi}[[\varphi]$.

Given a phaser program (B, P, Ts), we can characterize with a finite set of constraints all configurations involving $n$ tasks and $m$ phasers and satisfying the premises of rules (registration error), (assert. fault), (race) and (deadlock) listed in Figure 4.3.

Lemma 1 (Characterizing badness). Given a program (B, P, Ts) and natural numbers $(n, m)$, we can exhibit finite sets of constraints badCstrs sace $_{\text {ram }}^{(n, m)}$, badCstrs $\mathbf{a s s e r t}_{\text {ass }}^{(n, m)}$, badCstrs $\mathbf{s}_{\text {register }}^{(n, m)}$ and badCstrs $\mathbf{s}_{\text {deadlock }}^{(n, m)}$ such that:

$$
\begin{aligned}
\text { badConf } \mathbf{s}_{\text {race }}^{(n, m)} & \left.=\left[\text { badCstr } \mathbf{s}_{\text {race }}^{(n, m)}\right]\right] \\
\text { badConf } \mathbf{s}_{\text {assert }}^{(n, p)} & \left.=\left[\text { badCstr } \mathbf{s}_{\text {assert }}^{(n, m)}\right]\right] \\
\text { badConf } \mathbf{s}_{\text {register }}^{(n, p)} & \left.=\left[\text { badCstrs } \mathbf{s}_{\text {register }}^{(n, m)}\right]\right] \\
\text { badConf } \mathbf{s}_{\text {deadlock }}^{(n, p)} & \left.=\left[\text { badCstr } \mathbf{s}_{\text {deadlock }}^{(n, m)}\right]\right]
\end{aligned}
$$

In addition, we can choose the constraints in badCstrs $\mathbf{d e a d l o c k}_{(n, m)}$ to be of degree 0 while those in badCstrs $\mathbf{s}_{\text {race }}^{(n, m)}$, badCstrs $\mathbf{s}_{\text {assert }}^{(n, m)}$ or in badCstrs $\mathbf{s}_{\text {register }}^{(n, m)}$ to be free.

Proof. Observe that $n$ and $m$ are given natural numbers. Fix a task set $\mathcal{T}=\left\{t_{1}, \ldots, t_{n}\right\}$ of size $n$ and a phaser set $\mathcal{P}=\left\{p_{1}, \ldots, p_{m}\right\}$ of size $m$. We can therefore enumerate all tuples $(\mathcal{T}, \mathcal{P}, 6 v, p c, p v)$, where $6 v, p c$ and $p v$ are defined as in the configurations.

Let $\mathrm{C}$ be the set of such tuples. Observe that the tuples in $\mathrm{C}$ are missing information about tasks' registration and wait and signal values. We complete this information in the following. Given a set $\mathcal{U} \subseteq \mathcal{T}$ of tasks and a phaser $p \in \mathcal{P}$, we write topOf $(\mathcal{U}, p)$ to mean the graph of the conjunction $\bigwedge_{t, u \in \mathcal{U}}\left(\sigma^{t} \geq \omega^{u} \geq 0\right)$. Observe that an invariant of all phaser programs is that signal and wait phases (of all tasks registered with a given phaser) are always non-negative with the signal phases always greater than or equal to the wait phases. For this reason, top0f $(\mathcal{U}, p)$ is the weakest possible graph, where the set $\mathcal{U}$ is registered with a phaser $p$. Observe that top $0 f(\mathcal{U}, p)$ is free. We 
now finish the definitions of badCstrs $\mathbf{s}_{\text {race }}^{(n, m)}$, badCstrs $\mathbf{s}_{\text {assert }}^{(n, m)}$, badCstrs $\mathbf{s}_{\text {register }}^{(n, m)}$, badCstrs $\mathbf{s}_{\text {deadlock }}^{(n, m)}$ :

- Add to badCstrs $\mathbf{s}_{\text {race }}^{(n, m)}$ all constraints $(\mathcal{T}, \mathcal{P}, 6 v, p c, p v, \gamma)$, where:

- $(\mathcal{T}, \mathcal{P}, 6 v, p c, p v)$ is a tuple of $\mathrm{C}$ with two different tasks $t$ and $u$ in $\mathcal{T}$ executing a read or a write on a Boolean variable with at least one of them writing to it (see badConf $\mathrm{s}_{\text {race }}^{(n, m)}$ in Figure 4.3).

- The total mapping $\gamma$ associates top0f $\left(\mathcal{U}_{p}, p\right)$ to each phaser $p$, where $\mathcal{U}$ is some subset of $\mathcal{T}$. Intuitively, for each phaser $p$, we consider all registration possibilities (some subset $\mathcal{U} \subseteq \mathcal{T}$ ) while imposing the weakest possible constraints on the signal and wait phases of the registered tasks. Observe that $\gamma$ is free.

- Add to badCstrs $\mathbf{s}_{\text {assert }}^{(n, m)}$ all constraints $(\mathcal{T}, \mathcal{P}, 6 v, p c, p v, \gamma)$, where:

- $(\mathcal{T}, \mathcal{P}, 6 v, p c, p v)$ is a tuple of $\mathrm{C}$ with some task $t$ in $\mathcal{T}$ executing an assertion on a Boolean condition that evaluates to false with $6 v$ (see badConf $\mathrm{s}_{\text {assert }}^{(n, m)}$ in Figure 4.3).

- The total mapping $\gamma$ associates top0f $\left(\mathcal{U}_{p}, p\right)$ to each phaser $p$, where $\mathcal{U}$ is some subset of $\mathcal{T}$. Observe that $\gamma$ is free.

- Add to badCstrs $\mathbf{r}_{\text {register }}^{(n, m)}$ all constraints $(\mathcal{T}, \mathcal{P}, 6 v, p c, p v, \gamma)$, where:

- $(\mathcal{T}, \mathcal{P}, 6 v, p c, p v)$ is a tuple of $\mathrm{C}$ with some task $t$ in $\mathcal{T}$ executing a statement that involves a phaser variable ph.

- Again, the total mapping $\gamma$ associates top0f $\left(\mathcal{U}_{p}, p\right)$ to each phaser $p$, where $\mathcal{U}$ is some subset of $\mathcal{T}$. In addition, we require that either $p v(t)(\mathrm{ph}) \uparrow$ or $p=p v(t)(\mathrm{ph})$ with $t \notin \operatorname{Reg}(p, \gamma(p))$. (see badConf $\mathrm{s}_{\text {register }}^{(n, m)}$ in Figure 4.3). Observe $\gamma(q)$ is free for all $q$ on which $\gamma$ is defined. Hence, $\gamma$ is free.

- Add to badCstrs $\mathbf{s}_{\text {deadlock }}^{(n, m)}$ all constraints $(\mathcal{T}, \mathcal{P}, 6 v, p c, p v, \phi)$, where:

$-(\mathcal{T}, \mathcal{P}, 6 v, p c, p v)$ is a tuple of $\mathrm{C}$, where a set of tasks $t_{0}, \ldots, t_{l-1}$ in $\mathcal{T}$ are executing wait commands on phaser variables $\mathrm{ph}_{0}, \ldots, \mathrm{ph}_{l-1}$ in P.

- Again, the total mapping $\gamma$ associates top0f $\left(\mathcal{U}_{p}, p\right)$ to each phaser $p$, where $\mathcal{U}$ is some subset of $\mathcal{T}$. However, we require that: $p_{i}=$ $p v\left(t_{i}\right)\left(\mathrm{ph}_{i}\right)$ for each $i: 0 \leq i<l$ and, $t_{i}$ is waiting for $t_{(i+1) \% l}$ in $\gamma\left(p_{i}\right)$, i.e., $\gamma\left(p_{i}\right)$ imposes the wait phase of $t_{i}$ on $p_{i}$ is equal to the signal phase of $t_{(i+1) \% l}$ on $p_{i}$. In other words, the edge $\omega^{t_{i}} \stackrel{0}{\rightarrow}$ $\sigma^{t_{(i+1) \% l}}$ in $\gamma\left(p_{i}\right)$ (see badConf $\mathbf{s}_{\text {deadlock }}^{(n, m)}$ in Figure 4.3). Observe that the graphs in $\gamma$ are not all free since some of them put an 
upper bound on how large some signal values are compared to some wait values. They are however of degree 0 since the only negative weights are $-\infty$.

By construction, we have considered all possible tuples $(\mathcal{T}, \mathcal{P}, 6 v, p c, p v)$ and registration combinations. We have considered the weakest possible constraints on the phases for the registered tasks. Besides, any configuration in the denotation of the constraints will belong to the corresponding bad set.

\subsubsection{Entailment}

We say that a constraint $\varphi=(\mathcal{T}, \mathcal{P}, 6 v, p c, p v, \gamma)$ is weaker than another constraint $\varphi^{\prime}=\left(\mathcal{T}^{\prime}, \mathcal{P}^{\prime}, 6 v^{\prime}, p c^{\prime}, p v^{\prime}, \gamma^{\prime}\right)$, written $\varphi \sqsubseteq \varphi^{\prime}$, to mean the following. The two constraints have the same number of phasers and tasks. They agree on the values of the Boolean variables. Moreover, they agree on which tasks are registered with which phasers (up to a renaming on the values of the phaser variables). Also, the constraints on the wait and signal values are stronger in $\varphi^{\prime}$ than in $\varphi$. More formally, $\varphi \sqsubseteq \varphi^{\prime}$ if

- $6 v=6 v^{\prime}$,

- there are bijections $\kappa: \mathcal{T} \rightarrow \mathcal{T}^{\prime}$ and $\pi: \mathcal{P} \rightarrow \mathcal{P}^{\prime}$ such that for each $t \in \mathcal{T}$ and $p \in \mathcal{P}$ the following four conditions hold:

$$
\begin{aligned}
& -p c(t)=p c^{\prime}(\kappa(t)) ; \\
& -\pi(p v(t)(\mathrm{ph}))=p v^{\prime}(\kappa(t))(\mathrm{ph}) ; \\
& -\kappa(\operatorname{Reg}(p, \gamma(p)))=\operatorname{Reg}\left(\pi(p), \gamma^{\prime}(\pi(p))\right) ; \\
& -\gamma(p) \leq \mathcal{G} \gamma^{\prime}(\pi(p))\left[\left\{\omega^{t} / \omega^{\kappa(t)}, \sigma^{t} / \sigma^{\kappa(t)} \mid t \in \operatorname{Reg}(p, \gamma(p))\right\}\right] .
\end{aligned}
$$

Clearly, $\varphi \sqsubseteq \varphi^{\prime}$ implies $\left[\left[\varphi^{\prime}\right]\right] \subseteq[[\varphi]]$. We say that $\subseteq$ is sound. We can show that $\subseteq$ is a well-quasi-ordering over constraints of bounded degrees that involve fixed numbers of tasks and phasers. This is due to the fact that $\leq_{\mathcal{G}}$ is itself a well-quasi-ordering over graphs of bounded degrees over a finite set of variables $[113,100]$.

Lemma $2(\mathcal{W} \mathcal{Q O})$. Given $k, n, m \in \mathbb{N}$, the entailment relation $\sqsubseteq$ over the set of constraints of degree $k$ involving at most $n$ tasks and $m$ phasers is a well-quasi-ordering.

Proof. Assume an infinite sequence $\varphi_{1}, \varphi_{2}, \ldots$ of constraints, where $\varphi_{i}=$ $\left(\mathcal{T}_{i}, \mathcal{P}_{i}, b v_{i}\right.$, pc $_{i}$, gap $_{i}$, ega $\left._{i}\right)$, where $\left|\mathcal{T}_{i}\right|=n$ and $\left|\mathcal{P}_{i}\right|=m$ for all $i \geq 1$ and where all appearing graphs have degree $k$ or less. We can assume that $\mathcal{T}_{i}=\mathcal{T}_{j}$ and $\mathcal{P}_{i}=\mathcal{P}_{j}$ for any $i, j \geq 1$. We show there are $i<j$ such that $\varphi_{i} \sqsubseteq \varphi_{j}$. There is a finite number of different values for $6 v_{i}, p c_{i}, p v_{i}$ and for the domains of $\gamma_{i}(p)$ for each $p \in \mathcal{P}$. We can therefore extract an infinite subsequence, where: 
- $6 v_{i}=6 v_{j}, p c_{i}=p c_{j}, p v_{i}=p v_{j}$, and

- for each $p \in \mathcal{P}$, the domains of $\gamma_{i}(p)$ and $\gamma_{j}(p)$ coincide.

Observe that for each phaser $p \in \mathcal{P}$, the graphs $\gamma_{i}(p)$ are the same up to possible differences on the weights. Let $\leq$ be the component-wise-ordering on vectors. Each graph $\gamma_{i}(p)$ is of degree $k$ or less. We can therefore organize its weights as a vector vectorOf $\left(\gamma_{i}(p)\right)$ with elements in $\mathbb{Z} \cup\{-\infty,+\infty\}$ but where the only allowed negative elements are those larger than $-k$. The vectors can be organized in a way that vectorOf $\left(\gamma_{i}(p)\right) \leq$ vectorOf $\left(\gamma_{j}(p)\right)$ means $\gamma_{i}(p) \leq_{\mathcal{G}} \gamma_{j}(p)$. We then repeat the following steps for each $p \in \mathcal{P}$ : using Higman's lemma [72] and the degree boundedness of the constraints, we extract an infinite sequence of constraints, where vector0f $\left(\gamma_{a}(p)\right) \leq \operatorname{vector} 0 f\left(\gamma_{b}(p)\right)$ if $a<b$.

Theorem 8. The derivation rules of Figure 4.8 are sound.

Proof. Assume $c=(\mathcal{T}, \mathcal{P}, 6 v, p c, p v, \phi)$ and $\varphi=(\mathcal{T}, \mathcal{P}, 6 v, p c, p v, \gamma)$ such that $c \in[[\varphi]]$. By definition of $c \in[[\varphi]]$, tasks and phasers in $c$ and $\varphi$ are related to each other by two bijections. Hence, for brevity and without loss of generality, we assume that the tasks and phasers in $c$ and $\varphi$ are renamed so that they map to each other by the identity relation.

newPhaser. Assume $c^{\prime} \frac{\text { ph:=newPhaser() }}{t} c$ for some $c^{\prime}$ and assume $p$ is the new phaser id. Since $c \in[[\varphi]$ and $t$ is the only task registered with $p$ in $c$ with $\phi(p)(t)=(0,0)$, then, $\left(\omega^{t}=0, \sigma^{t}=0\right) \vDash \gamma(p)$ and $i \operatorname{sReg}(u, p, \gamma(p)) \Rightarrow u=t$ for each task $u \in \mathcal{T}$. We exhibit $\varphi^{\prime}$ such that $c^{\prime} \in\left[\left[\varphi^{\prime}\right]\right]$ and $\varphi \frac{\text { ph:=newPhaser }()}{t} \varphi^{\prime}$. Assuming $c^{\prime}=\left(\mathcal{T}, \mathcal{P}^{\prime}, 6 v, p c^{\prime}, p v^{\prime}, \phi^{\prime}\right)$, two cases are possible for $p v^{\prime}$. Either $p v^{\prime}(t)(\mathrm{ph})$ is undefined in which case the derivation rule newPhaser I captures $\varphi^{\prime}=\left(\mathcal{T}, \mathcal{P}^{\prime}, 6 v, p c^{\prime}, p v^{\prime}, \gamma \backslash\{p\}\right)$ that denotes $c^{\prime}$; or, $p v^{\prime}(t)(\mathrm{ph})$ associates the pair $(t, \mathrm{ph})$ to some phaser id $q \neq p$ which is captured by the newPhaser II derivation rule.

signal. Assume $\mathrm{c}^{\prime} \frac{\mathrm{ph} . \mathrm{signal}()}{t} \mathrm{c}$ for some $\mathrm{c}^{\prime}$, where $p v(t)(\mathrm{ph})=p$. We exhibit $\varphi^{\prime}$ such that $c^{\prime} \in\left[\left[\varphi^{\prime}\right]\right]$ and $\varphi \stackrel{\text { ph.signal }()}{t} \varphi^{\prime}$. The definition of $c \in[[\varphi]$ and the existence of $c^{\prime}$ ensure that $t$ is registered with $p$ in $\gamma(p)$. The signal phase of the task $t$ on the phaser $p$ in the configuration $c$ was obtained after incrementing the corresponding signal value in $c^{\prime}$. Therefore, the values denoted by $\sigma^{t}$ and $\omega^{u}$ in $\gamma(p)$ (for all $u$ registered with $p$ in c) can be restricted so that $\sigma^{t}>\omega^{u}$ for each $u$ registered with $p$ in $\gamma(p)$. $\gamma^{\prime}$ is obtained from $\gamma$ by enforcing this constraint and by decrementing the signal phase variable $\sigma^{t}$. Hence, if 


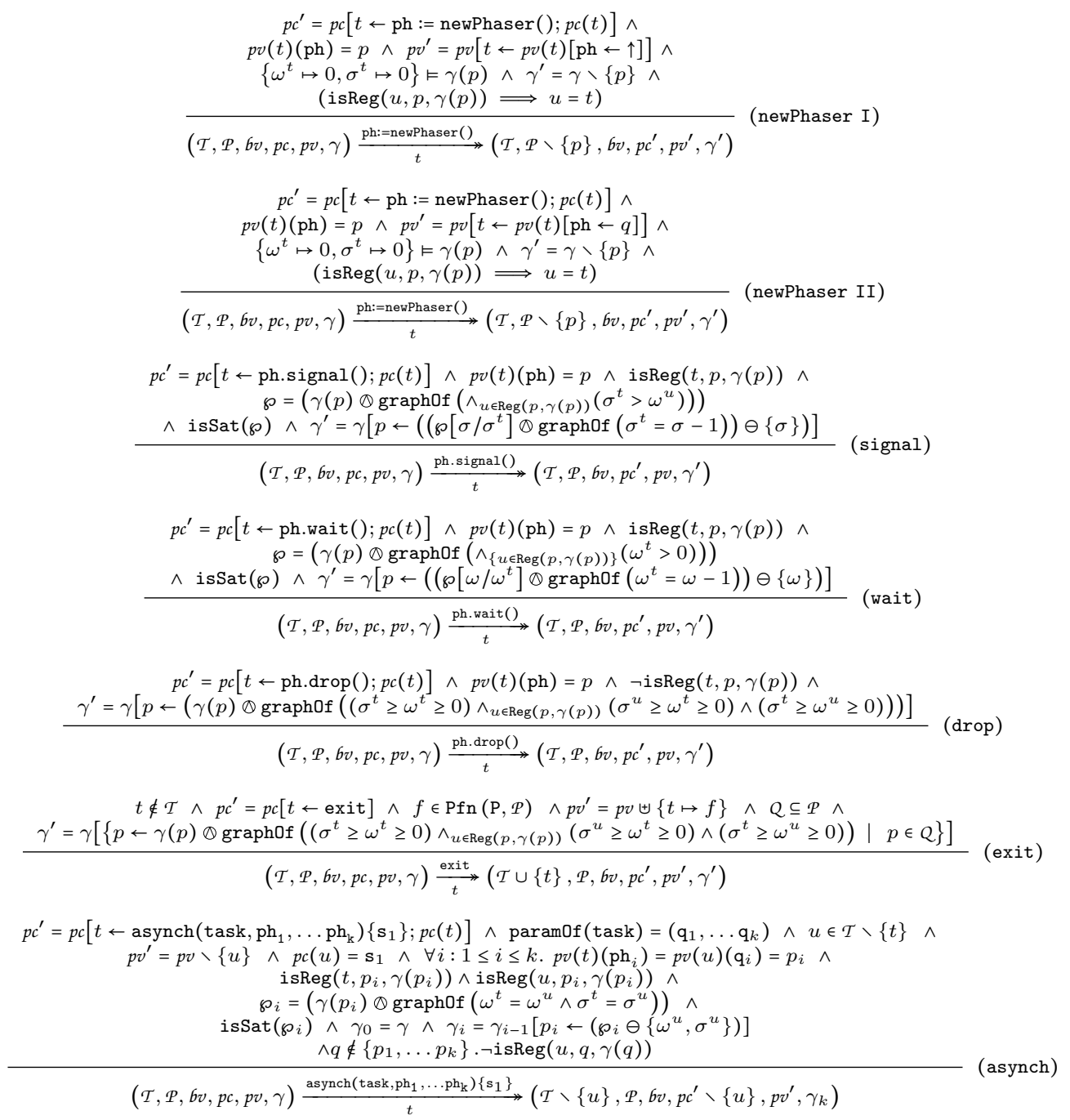

Figure 4.8: Derivation rules for computing $\operatorname{pre}_{\text {stmt }}(\varphi)$ as union of all $\left\{\varphi^{\prime} \mid \varphi \stackrel{\text { stmt }}{\longrightarrow} \varphi^{\prime}\right.$ with $\varphi=(\mathcal{T}, \mathcal{P}, 6 v, p c, p v, \gamma)$ and $\left.t \in \mathcal{T}\right\}$. 
$c^{\prime}=\left(\mathcal{T}, \mathcal{P}, 6 v, p c^{\prime}, p v, \phi^{\prime}\right)$, then, the constraint $\varphi^{\prime}=\left(\mathcal{T}, \mathcal{P}, 6 v, p c^{\prime}, p v, \gamma^{\prime}\right)$ denotes $\mathrm{c}^{\prime}$ and $\varphi \stackrel{\text { ph.signal }()}{\underset{t}{\longrightarrow}} \varphi^{\prime}$.

wait. Assume $c^{\prime} \stackrel{\text { ph.wait }()}{t} c$ for some $c^{\prime}$ where $p v(t)(\mathrm{ph})=p$. We exhibit $\varphi^{\prime}$ such that $c^{\prime} \in\left[\left[\varphi^{\prime}\right]\right]$ and $\varphi \stackrel{\text { ph.wait }()}{t} \varphi^{\prime}$. The definition of $c \in[[\varphi]$ and the existence of $c^{\prime}$ ensure that $t$ is registered with $p$ in $\gamma(p)$. The wait phase of the task $t$ on the phaser $p$ in the configuration $c$ was obtained after incrementing the corresponding wait value in $c^{\prime}$. The value denoted by $\omega^{t}$ in $\gamma(p)$ can therefore be restricted so that $\omega^{u}>0 . \quad \gamma^{\prime}$ is obtained from $\gamma$ by enforcing this constraint and by decrementing the wait phase variable $\omega^{t}$. Hence, if $c^{\prime}=\left(\mathcal{T}, \mathcal{P}, 6 v, p c^{\prime}, p v, \phi^{\prime}\right)$, then, the constraint $\varphi^{\prime}=\left(\mathcal{T}, \mathcal{P}, b v, p c^{\prime}, p v, \gamma^{\prime}\right)$ denotes $\mathrm{c}^{\prime}$ and $\varphi \stackrel{\text { ph.wait }()}{\underset{t}{\longrightarrow}} \varphi^{\prime}$.

drop. Assume $\mathrm{c}^{\prime} \underset{t}{\stackrel{\text { ph.drop }()}{\longrightarrow}} \mathrm{c}$ for some $\mathrm{c}^{\prime}$, where $p v(t)(\mathrm{ph})=p$. We exhibit $\varphi^{\prime}$ such that $c^{\prime} \in\left[\left[\varphi^{\prime}\right]\right]$ and $\varphi \stackrel{\text { ph.drop }()}{t} \varphi^{\prime}$. The definition of $c \in[[\varphi]]$ and the existence of $\mathrm{c}^{\prime}$ ensure that $t$ is not registered with $p$ in $\gamma(p)$. Let $\gamma^{\prime}$ capture the most relaxed constraint on wait and signal phases of the tasks registered with $p$, including $t$, and enforce the invariants of the phaser programs. Hence, if $c^{\prime}=\left(\mathcal{T}, \mathcal{P}, 6 v, p c^{\prime}, p v, \phi^{\prime}\right)$, then, the constraint $\varphi^{\prime}=\left(\mathcal{T}, \mathcal{P}, 6 v, p c^{\prime}, p v, \gamma^{\prime}\right)$ denotes $c^{\prime}$ and $\varphi \stackrel{\text { ph.drop }()}{\underset{t}{\longrightarrow}} \varphi^{\prime}$.

exit. Assume $c^{\prime} \underset{t}{\stackrel{\text { exit }}{\longrightarrow}} \mathrm{c}$ for some $c^{\prime}$. We exhibit $\varphi^{\prime}$ such that $c^{\prime} \in\left[\left[\varphi^{\prime}\right]\right]$ and $\varphi \stackrel{\text { exit }}{\longrightarrow} \varphi^{\prime}$. The definition of $c \in[\varphi]$ and the existence of $c^{\prime}$ ensure that $t$ is not a member of $\mathcal{T}$. If $c^{\prime}=\left(\mathcal{T}, \mathcal{P}, b v, p c^{\prime}, p v^{\prime}, \phi^{\prime}\right)$, then, let $\gamma^{\prime}$ be built with respect to a set $Q \subseteq \mathcal{P}$ representing phasers to which $t$ was registered in $\phi^{\prime}$. Let also $\gamma^{\prime}$ introduce the most relaxed wait and signal phases of $t$ to each phaser in $Q$. For other tasks, let $\gamma^{\prime}$ be the same as $\gamma$. Then, the constraint $\varphi^{\prime}=\left(\mathcal{T}, \mathcal{P}, 6 v, p c^{\prime}, p v^{\prime}, \gamma^{\prime}\right)$ denotes $c^{\prime}$ and $\varphi \underset{t}{\stackrel{\text { exit }}{\longrightarrow}} \varphi^{\prime}$.

asynch. Assume $c \in[[\varphi]]$ and $c^{\prime} \frac{\operatorname{asynch}\left(\operatorname{task}, \mathrm{ph}_{1}, \ldots \mathrm{ph}_{\mathrm{k}}\right)\left\{\mathbf{s}_{1}\right\}}{t} \mathrm{c}$ for some $\mathrm{c}^{\prime}$, where $\operatorname{param0f}($ task $)=\left(\mathrm{q}_{1}, \ldots \mathrm{q}_{k}\right)$. Task $t$ has just spawned the task $u$ in $\mathrm{c}$. We exhibit $\varphi^{\prime}$ such that $c^{\prime} \in\left[\left[\varphi^{\prime}\right]\right]$ and $\varphi \frac{\operatorname{asynch}\left(\operatorname{task}, \mathrm{ph}_{1}, \ldots \mathrm{ph}_{\mathrm{k}}\right)\left\{\mathbf{s}_{1}\right\}}{t} \varphi^{\prime}$. The definition of $\mathrm{c} \in[[\varphi]]$ ensures that for each $i: 1 \leq i \leq k, p v(t)\left(\mathrm{ph}_{i}\right)=$ $p v(u)\left(q_{i}\right)=p_{i}$ and that $\gamma$ can be constrained so that $t$ and $u$ are registered with each $p_{i}$ in $\gamma$ and have the same phases. Assume $\gamma^{\prime}$ is ob- 
tained from $\gamma$ by imposing those constraints and removing the phase variables of $u$ on every phaser $p_{i}$. If $c^{\prime}=\left(\mathcal{T}^{\prime}, \mathcal{P}, b v, p c^{\prime}, p v^{\prime}, \phi^{\prime}\right)$, the constraint $\varphi^{\prime}=\left(\mathcal{T}^{\prime}, \mathcal{P}, 6 v, p c^{\prime}, p v^{\prime}, \gamma^{\prime}\right)$ denotes $c^{\prime}$. Moreover, by definition of the asynch derivation rule, $\varphi \stackrel{\text { asynch }\left(\operatorname{task}, \mathrm{ph}_{1}, \ldots \mathrm{ph}_{\mathrm{k}}\right)\left\{\mathbf{s}_{1}\right\}}{t} \varphi^{\prime}$.

Theorem 9. The derivation rules of Figure 4.8 are complete.

Assume $\varphi^{\prime}=(\mathcal{T}, \mathcal{P}, 6 v, p c, p v, \gamma)$ is obtained from $\varphi$ using a rule in Figure 4.8. We exhibit for any $c^{\prime} \in\left[\left[\varphi^{\prime}\right]\right]$ a configuration $c \in[[\varphi]]$ such that $c$ is a successor of $c^{\prime}$ according to the semantics of the phaser programs introduced in Figure 4.3.

By definition of $c^{\prime} \in\left[\left[\varphi^{\prime}\right]\right.$, tasks and phasers in $c^{\prime}$ and $\varphi^{\prime}$ are related to each other by two bijections. Hence, for brevity and without loss of generality, we assume that the tasks and phasers in $c^{\prime}$ and $\varphi^{\prime}$ are renamed so that they map to each other by the identity relation.

newPhaser. Assume $\varphi \frac{\mathrm{ph}:=\text { newPhaser }()}{t} \varphi^{\prime}$ for some $\varphi$ and assume $p$ is the new phaser id. We exhibit $c$ such that $c \in[[\varphi]]$ and $c^{\prime} \frac{\text { ph:=newPhaser( })}{t} c$. Let $\phi^{\prime}$ be obtained from $\phi$ by associating the pair $(p, t)$ to the phases $(0,0)$, i.e., $\phi^{\prime}(p)(t)=(0,0)$. Therefore, if $\varphi=\left(\mathcal{T}, \mathcal{P}^{\prime}, 6 v, p c^{\prime}, p v^{\prime}, \gamma^{\prime}\right)$, then the configuration $c=\left(\mathcal{T}, \mathcal{P}^{\prime}, 6 v, p c^{\prime}, p v^{\prime}, \phi^{\prime}\right)$ satisfies both $\left.c \in[\varphi \varphi]\right]$ and $c^{\prime} \frac{\text { ph:=newPhaser }()}{t} c$.

signal. Assume $\varphi \stackrel{\text { ph.signal }()}{t} \varphi^{\prime}$ for some $\varphi$, where $p v(t)(\mathrm{ph})=p$. We exhibit $c$ such that $c \in \llbracket \varphi \rrbracket$ and $c^{\prime} \frac{\text { ph.signal }()}{t} c$. Assuming $\varphi=$ $\left(\mathcal{T}, \mathcal{P}, 6 v, p c^{\prime}, p v, \gamma^{\prime}\right)$, the configuration $\mathrm{c}=\left(\mathcal{T}, \mathcal{P}, 6 v, p c^{\prime}, p v, \phi^{\prime}\right)$, where $\phi^{\prime}$ captures incrementing the signal phase $\operatorname{sig}_{p}^{t}$, satisfies both $c \in \llbracket \varphi \rrbracket$ and $\mathrm{C}^{\prime} \underset{t}{\stackrel{\text { ph.signal }()}{\longrightarrow}} \mathrm{C}$.

wait. Assume $\varphi \stackrel{\text { ph.wait }()}{t} \varphi^{\prime}$ for some $\varphi$, where $p v(t)(\mathrm{ph})=p$. We exhibit c such that $\mathrm{c} \in[[\varphi]]$ and $\mathrm{c}^{\prime} \stackrel{\text { ph.signal }()}{t} \mathrm{c}$. Assuming $\varphi=\left(\mathcal{T}, \mathcal{P}, 6 v, p c^{\prime}, p v, \gamma^{\prime}\right)$, the configuration $c=\left(\mathcal{T}, \mathcal{P}, 6 v, p c^{\prime}, p v, \phi^{\prime}\right)$, where $\phi^{\prime}$ captures incrementing the wait phase $w_{a i t}^{t}$, satisfies both $\mathrm{c} \in[[\varphi]]$ and $\mathrm{c}^{\prime} \frac{\text { ph.wait }()}{t} \mathrm{c}$.

drop. Assume $\varphi \stackrel{\text { ph.drop }()}{t} \varphi^{\prime}$ for some $\varphi$, where $p v(t)(\mathrm{ph})=p$. We exhibit $\mathrm{c}$ such that $c \in[[\varphi]]$ and $c^{\prime} \stackrel{\text { ph.drop }()}{t} c$. Assuming $\varphi=\left(\mathcal{T}, \mathcal{P}, 6 v, p c^{\prime}, p v^{\prime}, \gamma^{\prime}\right)$. Let 
$p v^{\prime}$ and $\phi^{\prime}$ respectively be obtained from $p v$ and $\phi$ by removing $t$ from $p v$ and $\phi(p)$. Then, the configuration $c=\left(\mathcal{T}, P, b v, p c^{\prime}, p v^{\prime}, \phi^{\prime}\right)$ satisfies both $c \in[[\varphi]]$ and $\mathrm{c}^{\prime} \stackrel{\text { ph.drop }()}{\underset{t}{\longrightarrow}} \mathrm{c}$.

exit. Assume $\varphi \stackrel{\text { ph.drop }()}{t} \varphi^{\prime}$ for some $\varphi$, where $p v(t)(\mathrm{ph})=p$. We exhibit c such that $c \in[[\varphi]]$ and $c^{\prime} \stackrel{\text { ph.drop() }}{t}$ c. Assuming $\varphi=\left(\mathcal{T}^{\prime}, \mathcal{P}, 6 v, p c^{\prime}, p v^{\prime}, \gamma^{\prime}\right)$, where $\mathcal{T}^{\prime}=\mathcal{T} \backslash\{t\}$ and $p v^{\prime}$ and $\phi^{\prime}$ are respectively obtained by restricting $p v$ and $\phi$ to $\mathcal{T}^{\prime}$, the configuration $c=\left(\mathcal{T}, \mathcal{P}, 6 v, p c^{\prime}, p v^{\prime}, \phi^{\prime}\right)$ satisfies both $c \in[[\varphi]]$ and $c^{\prime} \stackrel{\text { ph.drop() }}{t}$ c.

asynch. Assume $\varphi \stackrel{\text { asynch }\left(\mathrm{task}_{\mathrm{ph}}, \ldots \mathrm{ph}_{\mathrm{k}}\right)\{\mathrm{s}\}}{t} \underset{t}{\longrightarrow} \varphi^{\prime}$ for some $\varphi$, where $\operatorname{param0f}($ task $)=\left(q_{1}, \ldots q_{k}\right)$. We exhibit $\mathrm{c}$ such that $\mathrm{c} \in[[\varphi]]$ and $\mathrm{c}^{\prime} \underset{t}{\stackrel{\operatorname{asynch}\left(\operatorname{task} \mathrm{ph}_{1}, \ldots \mathrm{ph}_{\mathrm{k}}\right)\{\mathrm{s}\}}{\longrightarrow}}$ c. Assuming $\varphi=\left(\mathcal{T}^{\prime}, \mathcal{P}, 6 v, p \mathcal{c}^{\prime}, p v^{\prime}, \gamma^{\prime}\right)$, the configuration $\mathrm{c}=\left(\mathcal{T}^{\prime}, \mathcal{P}, 6 v, p c^{\prime}, p v^{\prime}, \phi^{\prime}\right)$, where for each $i: 1 \leq i \leq k$, $p v^{\prime}(t)\left(\mathrm{ph}_{i}\right)=p v^{\prime}(u)\left(\mathrm{q}_{i}\right)=p_{i}$, and in $\phi^{\prime}$ the tasks $t$ and $u$ have the same phase on every phaser $p_{i}$, satisfies both $\mathrm{c} \in[[\varphi]]$ and $\mathrm{c}^{\prime} \frac{\text { asynch }\left(\operatorname{task}, \mathrm{ph}_{1}, \ldots \mathrm{ph}_{\mathrm{k}}\right)\{\mathbf{s}\}}{t} \mathrm{c}$.

\subsection{Verification Procedure}

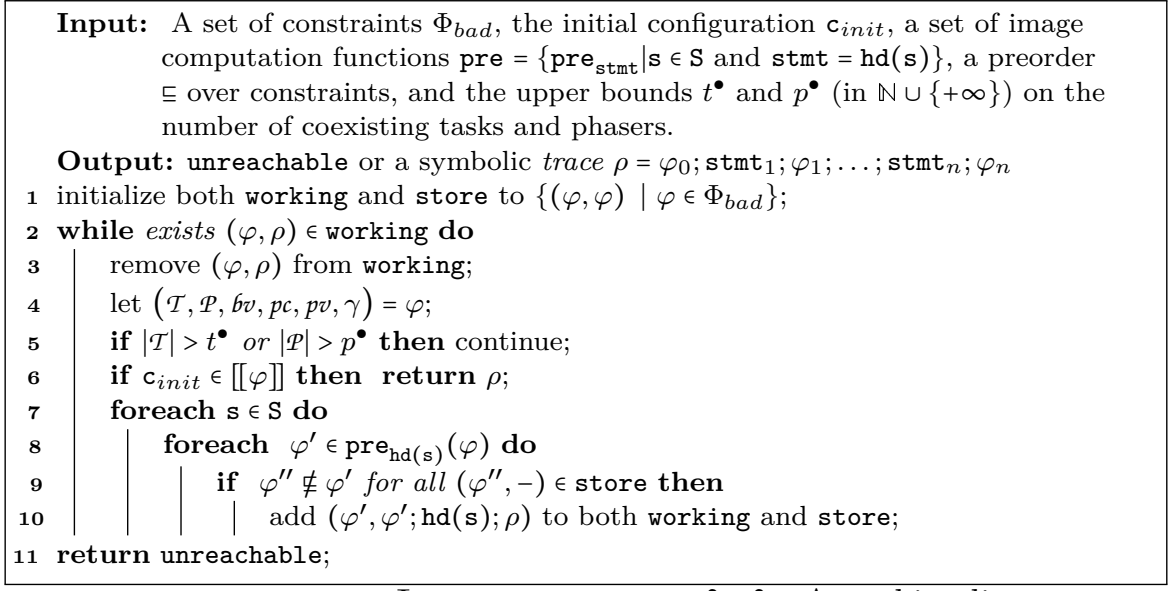

Procedure phaser-explore $\left(\Phi_{b a d}, \mathrm{c}_{i n i t}, \mathrm{~S}, \mathrm{pre}, \sqsubset, t^{\bullet}, p^{\bullet}\right)$. A working-list procedure to check whether some bad configuration denoted by constraints in $\Phi_{b a d}$ is reachable from the initial configuration $c_{i n i t}$. The procedure

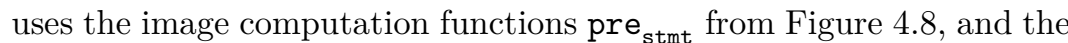
preorder $\sqsubseteq$ on the constraints. The upper bounds $t^{\bullet}$ and $p^{\bullet}$ determine the maximum numbers of tasks and phasers in every constraint. 
We adapt the reachability analysis introduced in Section 3.3 in backward to analyse the transition systems induced by phaser programs. The only difference compared to that procedure is that we have added line 5 to discard those constraints that do not respect the upper bounds on the number of tasks or phasers. The procedure starts from a set of constraints $\Phi_{b a d}$. Its goal is to check if there is a trace from any of those constraints to some constraint whose denotation intersects some initial configuration in $c_{i n i t}$ using the set of

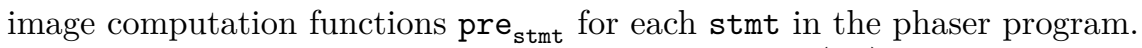

$\Phi_{b a d}$ can, for instance, be any subset of badCstrs $\mathbf{s}_{\text {deadlock }}^{(n, p)}$ (degree 0) or of badCstrs $\mathbf{s}_{\text {assert }}^{(n, p)}$ (free) in case we want to check the possibility of a deadlock or of an assertion violation.

Lemma 3 (Partial correctness). If the procedure "phaserexplore" $\left(\Phi_{b a d}, \mathrm{c}_{\text {init }}, \mathrm{S}, \mathrm{pre}\right.$, ㄷ, $\left.t^{\bullet}, p^{\bullet}\right)$ in Section 4.6 returns unreachable, then $\mathrm{c}_{\text {init }} \stackrel{*}{\rightarrow}\left[\left[\Phi_{\text {bad }}\right]\right]$. If it returns a trace $\varphi_{n}$, stmt $_{n}, \ldots$, stmt $_{1}, \varphi_{1}$, then, there are $\mathrm{c}_{n}, \ldots \mathrm{c}_{1}$ with $\mathrm{c}_{n}=\mathrm{c}_{\text {init }}, \mathrm{c}_{1} \in\left[\left[\Phi_{\text {bad }}\right]\right]$ and $\mathrm{c}_{i} \stackrel{\text { stmt }_{i}}{\longrightarrow} \mathrm{c}_{i-1}$ for $i: 1<i \leq n$.

Proof. The procedure "phaser-explore" $\left(\Phi_{b a d}, \mathrm{c}_{\text {init }}, \mathrm{S}, \mathrm{pre}, \sqsubset, t^{\bullet}, p^{\bullet}\right)$ with an unbounded number of tasks and phasers is an instantiation of the procedure "explore" in Section 3.3. By soundness and completeness of pre (respectively, Theorems 8 and 9), and by soundness and completeness of the procedure "explore" (respectively, Theorems 4 and 5), the procedure "phaserexplore" $\left(\Phi_{b a d}, \mathrm{c}_{\text {init }}, \mathrm{S}\right.$, pre, ᄃ) is sound and complete. However, since $\sqsubseteq$ is not $\mathcal{W Q O}$ for an unbounded number of tasks and phasers, the procedure "phaserexplore" might not terminate. Hence, it is partially exact.

Theorem 10 (Free termination). The procedure "phaserexplore" $\left(\Phi_{\text {bad }}, \mathrm{c}_{\text {init }}, \mathrm{S}\right.$, pre, $\left.,, t^{\bullet}, p^{\bullet}\right)$ terminates for $t^{\bullet}, p^{\bullet} \in \mathbb{N}$ and free $\Phi_{\text {bad }}$.

Proof. $\sqsubseteq$ is $\mathcal{W} \mathcal{Q O}$ over the degree-0 constraints with a bounded number of tasks and phasers. Moreover, freeness is preserved by the pre computation (Figure 4.8). Hence, by Theorem 6 we conclude the procedure "phaserexplore" terminates.

In order to check the reachability of arbitrary constraints, we may need to force termination. We do this by soundly bounding the degree of generated constraints using a relaxation $\varrho_{k}$. The relaxation $\varrho_{l}((\mathcal{T}, \mathcal{P}, 6 v, p c, p v, \gamma))$ replaces, in each graph $\gamma(p)$, each weight $k^{\prime \prime}$ such that $k^{\prime \prime}<-k$ with $-\infty$.

1 foreach $\varphi^{\prime \prime} \in \operatorname{pre}(t, \varphi)$ do

$2 \quad$ Let $\varphi^{\prime}=\varrho_{k}\left(\varphi^{\prime \prime}\right)$; 
Theorem 11 (Forced termination). Independently of the degree of $\Phi_{b a d}$, procedure "phaser-explore" $\left(\Phi_{\text {bad }}, \mathrm{c}_{\text {init }}, \mathrm{S}\right.$, pre $\left., \sqsubset, t^{\bullet}, p^{\bullet}\right)$, where line 8 is replaced by the two lines of Figure 6 , is sound and guaranteed to terminate.

Proof. Soundness is due to the validity of $\varrho_{k}(\varphi) \sqsubseteq \varphi$ while the termination argument relies, similarly to Theorem 10, on well-quasi orderedness of $\subseteq$ on the set of constraints with bounded degree and fixed numbers of tasks and phasers.

\subsection{Experimental Results}

We report on experiments with our open source prototype PhaserVerify ${ }^{1}$ for the verification of phaser programs. We conducted experiments on 12 different programs (some of which are from [40]). We considered both deadlock and assertion reachability problems. For each property, we considered correct and buggy versions. This resulted in 48 different instances with 2 to 3 phasers and 2 to 4 tasks. Our tool uses global phaser and task variables as in [40]. Experiments were conducted on an Intel Core(TM) i7 processor running at $2.9 \mathrm{GHz}$ with $8 \mathrm{~GB}$ of memory. Our implemented procedure does not eagerly concretize the states of the tasks described in the predecessor computation of Chapter 4.5. Instead, we collect conditions on the phases of the tasks that have not yet take any actions and lazily concretize them. Reported times for checking deadlocks are the sums of the times required to check reachability for each cycle. The prototype is only a proof of concept. For instance, the example (10.Running-3) is a variant of (09-Running-2), where a task instance is spawned twice, leading to two symmetrical tasks (out of four). This required up to three orders of magnitude more time to check than (09-Running-2). We believe partial order reduction techniques would help here. Other relevant heuristics would be to make use of priority queues and to organize the minimal sets. All examples are available on the tool homepage.

\subsection{Conclusion}

We have proposed a gap-order based reachability analysis for phaser programs. We have shown our analysis to be exact and guaranteed to terminate when checking registration, race, and assertion errors. We have established the undecidability of deadlock verification and explained how to turn our analysis into a sound over-approximation. In the next chapter, we discuss the parameterized verification of phaser programs.

\footnotetext{
${ }^{1}$ https://gitlab.liu.se/live/phaserverify
} 


\begin{tabular}{|c|ccc|}
\hline program & property & safe / buggy & times \\
\hline \hline \multirow{2}{*}{ 01.Loopless } & deadlock: & ok / trace & $1 \mathrm{~s} / 1 \mathrm{~s}$ \\
\cline { 2 - 4 } & assertion: & ok / trace & $1 \mathrm{~s} / 1 \mathrm{~s}$ \\
\hline \hline \multirow{2}{*}{$\begin{array}{c}\text { 02.Iterative } \\
\text { averaging }\end{array}$} & deadlock: & ok / trace & $1 \mathrm{~s} / 1 \mathrm{~s}$ \\
\cline { 2 - 4 } & assertion: & ok / trace & $1 \mathrm{~s} / 1 \mathrm{~s}$ \\
\hline \hline \multirow{2}{*}{$\begin{array}{c}\text { p3.Ordered } \\
\text { phasers }\end{array}$} & deadlock: & ok / trace & $1 \mathrm{~s} / 1 \mathrm{~s}$ \\
\cline { 2 - 4 } & assertion: & ok / trace & $13 \mathrm{~s} / 1 \mathrm{~s}$ \\
\hline \hline \multirow{2}{*}{ 04.Conditional } & deadlock: & ok / trace & $2 \mathrm{~s} / 1 \mathrm{~s}$ \\
\cline { 2 - 4 } & assertion: & ok / trace & $4 \mathrm{~s} / 7 \mathrm{~s}$ \\
\hline \hline \multirow{2}{*}{ 05.Loop Synch. } & deadlock: & ok / trace & $178 \mathrm{~s} / 145 \mathrm{~s}$ \\
\cline { 2 - 4 } & assertion: & ok / trace & $7 \mathrm{~s} / 13 \mathrm{~s}$ \\
\hline \hline \multirow{2}{*}{ 06.Nested forks } & deadlock: & ok / trace & $2 \mathrm{~s} / 1 \mathrm{~s}$ \\
\cline { 2 - 4 } & assertion: & ok / trace & $1 \mathrm{~s} / 1 \mathrm{~s}$ \\
\hline \hline \multirow{2}{*}{$\begin{array}{c}\text { 07.Conditional } \\
\text { membership }\end{array}$} & deadlock: & ok / trace & $1 \mathrm{~s} / 1 \mathrm{~s}$ \\
\cline { 2 - 4 } & assertion: & ok / trace & $12 \mathrm{~s} / 3 \mathrm{~s}$ \\
\hline \hline \multirow{2}{*}{$\begin{array}{c}\text { 08.Producer- } \\
\text { consumer }\end{array}$} & deadlock: & ok / trace & $37 \mathrm{~s} / 222 \mathrm{~s}$ \\
\cline { 2 - 4 } & assertion: & ok / trace & $79 \mathrm{~s} / 34 \mathrm{~s}$ \\
\hline \hline \multirow{2}{*}{ 09.Running-2 } & deadlock: & ok / trace & $5 \mathrm{~s} / 1 \mathrm{~s}$ \\
\cline { 2 - 4 } & assertion: & ok / trace & $26 \mathrm{~s} / 4 \mathrm{~s}$ \\
\hline \hline \multirow{2}{*}{ 10.Running-3 } & deadlock: & ok / trace & $4318 \mathrm{~s} / 128 \mathrm{~s}$ \\
\cline { 2 - 4 } & assertion: & ok / trace & $18631 \mathrm{~s} / 54 \mathrm{~s}$ \\
\hline
\end{tabular}

Table 4.1: The results of checking assertion violation and deadlock of Phaser programs with PhaserVerify 



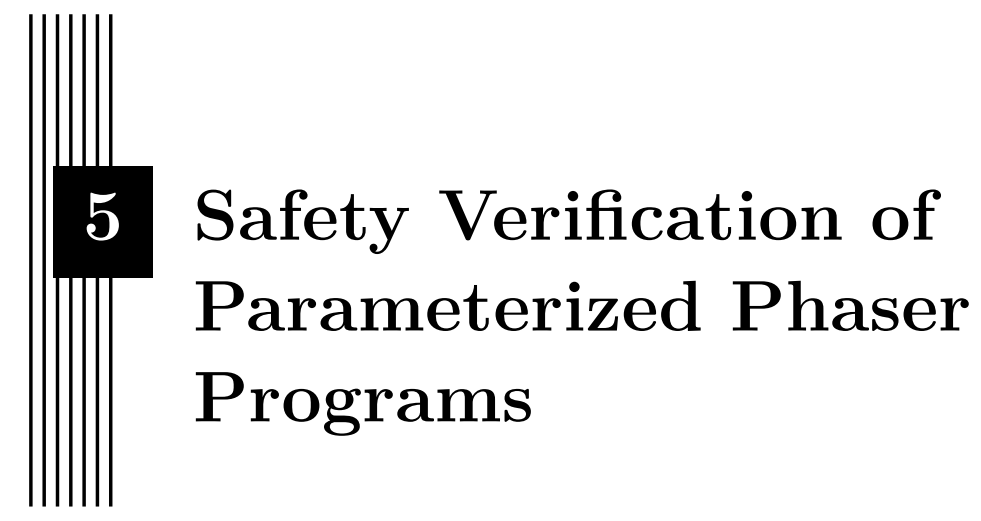

In this chapter, we address the problem of statically checking safety properties (such as assertions or deadlocks) for parameterized phaser programs. We consider programs that may generate arbitrarily many tasks and phasers. We study different formulations of the verification problem and propose an exact procedure that is guaranteed to terminate for some reachability problems even in the presence of unbounded phases and arbitrarily many spawned tasks. In addition, we prove undecidability results for several problems. Finally, we report on our experimental results.

\subsection{Introduction}

We focus on the parameterized verification problem of parallel programs that adopt the phaser construct for synchronization [115]. Parameterized verification of phaser programs is a challenging problem due to the arbitrary numbers of involved tasks and phasers.

We build on the operational model for phaser programs introduced in Chapter 4 and consider an additional statement called next. This statement consists of a signal followed by a wait. The next command may be associated with a sequence of statements that are to be executed in isolation by one of the registered tasks participating in the command. We call a program that does not use this feature of the next statement non-atomic.

This chapter provides general (un)decidability results that we believe will guide verification of other synchronization constructs. We identify combina- 
tions of features (e.g., unbounded differences between signal and wait phases, atomic statements) and properties to be checked (e.g., assertions, deadlocks) for which the parameterized verification problem becomes undecidable. These help identify synchronization constructs with enough expressivity to result in undecidable parameterized verification problems. We also provide a symbolic verification procedure that even terminates on fragments with arbitrary phases and numbers of spawned tasks. We get back to possible implications in the conclusion section. We summarize our contributions as follows:

- We propose an exact symbolic verification procedure for checking reachability of sets of configurations for non-atomic phaser programs when only distinguished tasks such as main create phasers. Observe that in such programs arbitrarily many tasks and phasers may be generated.

- We prove undecidability results for several reachability problems.

- We show termination of our procedure when checking assertions for nonatomic programs, even when the programs may spawn arbitrarily many tasks.

- We show termination of our procedure when checking deadlock-freedom and assertions for non-atomic programs in which the difference between signal and wait phases is bounded, even when the programs may spawn arbitrarily many tasks.

Related work. The closest work to ours is [62], which was introduced in Chapter 4 and is the only work on automatic and static formal verification of phaser programs. However, the work in [62] considers a bounded number of tasks and phasers. The approach introduced in this chapter can decide whether some program assertion has been violated even in the presence of arbitrarily many tasks with arbitrarily large phase gaps. This is well beyond [62] and requires a more complex symbolic representation with a more profound termination argument.

Outline. We describe the phaser construct in Section 5.2. We then formally introduce the construct and show that the associated general reachability problem is undecidable in Section 5.3. In Section 5.4, we describe our symbolic representation and state some of its non-trivial properties. We use the representation in Section 5.5 to define a verification procedure and establish decidability results. We refine our undecidability results in Section 5.6. We summarize our findings in Section 5.7. 
ph.next()\{stmt $\}$. We also let arbitrarily many instances of each task be spawned during program execution. Arbitrarily many phasers might also be generated during program execution.

$$
\text { stmt }::=\ldots|\operatorname{ph} \cdot \operatorname{next}() ;| \operatorname{ph} . \operatorname{next}()\{\text { stmt }\}
$$

ph.next() is syntactic sugar for a signal followed by a wait. ph.next ()$\{$ stmt $\}$ is similar to ph.next(). However, the block of code stmt is executed atomically by precisely one of the tasks participating in the synchronization before all tasks continue the execution that follows the barrier. ph.next ()$\{$ stmt $\}$, thus, requires all tasks to be synchronized on the same statement and is less flexible. The absence of a ph.next()\{stmt\} makes a program non-atomic. Note that the assignment of phaser variables is excluded from the syntax; additionally, we restrict task creation asynch(task, $\left.\mathrm{ph}_{1}, \ldots, \mathrm{ph}_{\mathrm{n}}\right)$ and require that the parameter variables $\mathrm{ph}_{i}$ are all different. This prevents two variables of a single task from referencing the same phaser and avoids the need to deal with aliasing: we can reason on the single variable in a process that references a phaser. Extending our work to deal with aliasing would be easy but would require heavier notations.

We will need the notions of configurations, partial configurations, and inclusion to define the reachability problems we consider in this work. We introduce them in the following and assume a phaser program $\operatorname{Pr}=(B, P, T s)$.

Configurations. The configurations of a phaser program describe valuations of its variables, control sequences of its tasks, and registration details to the phasers.

Control sequences. The set of control sequences $\mathrm{S}$ is defined similar to Section 4.4. But, it also contains (i) all $\mathrm{s}_{1} ;$ ph.next ()\{\}$; \mathrm{tl}(\mathrm{s})$ if $\mathrm{s}$ is in $\mathrm{S}$ and $\operatorname{hd}(\mathrm{s})=\operatorname{ph} . \operatorname{next}()\left\{\mathrm{s}_{1}\right\}$; and (ii) all ph.signal(); ph.wait();tl(s) if $\mathrm{s}$ is in $\mathrm{S}$ and $\operatorname{hd}(\mathrm{s})=\operatorname{ph} \cdot \operatorname{next}()\{\}$.

Partial configurations. Partial configurations allow the characterization of sets of configurations by partially stating some of their common characteristics. We only consider configurations that have only one instance of the task main. A partial configuration $\mathrm{c}$ of $\operatorname{Pr}=(\mathrm{B}, \mathrm{P}, \mathrm{Ts})$ is a tuple $(\mathcal{T}, \mathcal{P}, 6 v, p c$, phase $)$ where:

- $\mathcal{T}$ is a finite set of task identifiers. We let $t, u$ range over $\mathcal{T}$.

- $\mathcal{P}$ is a finite set of phaser identifiers. We let $p, q$ range over $\mathcal{P}$.

- $6 v: \mathrm{B} \rightarrow \mathbb{B} \cup\{*\}$ fixes the values of some of the shared variables.

- $p c: \mathcal{T} \rightarrow \mathrm{S} \cup\{*\}$ fixes the control sequences of some of the tasks. 
- phase : $\mathcal{T} \rightarrow \operatorname{Pfn}\left(\mathcal{P}, \mathrm{P} \cup\{-, *\} \times\left(\mathbb{N}^{2} \cup\{(*, *)\right.\right.$, nreg $\left.\left.\}\right)\right)$ is a mapping that associates to each task $t$ in $\mathcal{T}$ a partial mapping stating which phasers are known by the task and with which registration values.

Intuitively, partial configurations are used to state some facts about the valuations of variables and the control sequences of tasks and their registrations. Partial configurations leave some details unconstrained using partial mappings or the symbol $*$. For instance, if $6 v\left(v^{b}\right)=*$ in a partial configuration $(\mathcal{T}, \mathcal{P}, 6 v, p c$, phase $)$, then the partial configuration does not constrain the value of the shared variable $\mathrm{v}^{b}$. Moreover, a partial configuration does not constrain the relation between a task $t$ and a phaser $p$ when prase $(t)(p)$ is undefined. Instead, when the partial mapping phase $(t)$ is defined on phaser $p$, it associates a pair phase $(t)(p)=(\mathrm{v}, \mathrm{val})$ to $p$. If $\mathrm{v} \in \mathrm{P} \cup\{-, *\}$ is a variable $\mathrm{ph} \in \mathrm{P}$ then the task $t$ in $\mathcal{T}$ uses its variable ph to refer to the phaser $p$ in $\mathcal{P}^{1}$. If $\mathrm{v}$ is the symbol - then the task $t$ does not refer to ph with any of its variables in $\mathrm{P}$. If $\mathrm{v}$ is the symbol $*$, then the task might or might not refer to $p$. The value val in phase $(t)(p)=(\mathrm{v}, \mathrm{val})$ is either the value nreg or a pair (wait,sig). The value nreg means the task $t$ is not registered with phaser p. The pair (wait, sig) belongs to $(\mathbb{N} \times \mathbb{N}) \cup\{(*, *)\}$. In this case, task $t$ is registered with phaser $p$ with a symbolic wait phase wait and a symbolic signal phase sig. The value $*$ means that the wait phase wait (respectively signal phase sig) can be any value in $\mathbb{N}$. For instance, phase $(t)(p)=(\mathrm{ph}, \mathrm{nreg})$ means variable ph of the task $t$ refers to phaser $p$ but the task is not registered with $p$. On the other hand, phase $(t)(p)=(-,(*, *))$ means the task $t$ does not refer to $p$ but is registered with it with an arbitrary wait and signal phases.

Concrete configurations. A concrete configuration (or configuration for short) is a partial configuration $(\mathcal{T}, \mathcal{P}, 6 v, p c$, phase) where phase $(t)$ is total for each $t \in \mathcal{T}$ and where the symbol $*$ does not appear in any range. It is a tuple $(\mathcal{T}, \mathcal{P}, 6 v, p c$, phase $)$ where $6 v: \mathrm{B} \rightarrow \mathbb{B}, p c: \mathcal{T} \rightarrow \mathrm{S}$, and phase $: \mathcal{T} \rightarrow$ $\operatorname{Fn}(\mathcal{P}, \mathrm{P} \cup\{-\} \times((\mathbb{N} \times \mathbb{N}) \cup\{$ nreg $\}))$.

For a concrete configuration $(\mathcal{T}, \mathcal{P}, 6 v, p c$, phase $)$, we write isReg (phase, $t, p)$ to mean the predicate phase $(t)(p) \notin(\mathrm{P} \cup\{-\} \times\{\mathrm{nreg}\})$. The predicate isReg (phase, $t, p)$ captures whether the task $t$ is registered with phaser $p$ according to the mapping phase.

Note that a concrete configuration $c=(\mathcal{T}, \mathcal{P}, 6 v, p c$, phase $)$ in this chapter captures the same information as a configuration $c^{\prime}=(\mathcal{T}, \mathcal{P}, 6 v, p c, p v, \phi)$ in Chapter 4 if phase $(t)(p)=(\mathrm{ph}, \phi(t)(p))$ and $p v(t)(\mathrm{ph})=p$ for all $t \in \mathcal{T}$ and $p \in \mathcal{P}$. The differences in the configuration representations in the chapters are to simplify the presentation in each chapter. Another difference is that in this chapter we do not allow aliasing for the phaser variables in order to further simplify the presentation.

\footnotetext{
${ }^{1}$ The uniqueness of this variable is due to the absence of aliasing discussed before.
} 
Inclusion of configurations. A configuration $c^{\prime}=\left(\mathcal{T}^{\prime}, \mathcal{P}^{\prime}, 6 v^{\prime}, p c^{\prime}, p h a s e^{\prime}\right)$ includes a partial configuration $\mathrm{c}=(\mathcal{T}, \mathcal{P}, 6 v, p c$, phase $)$ if renaming and deleting tasks, phasers, or registrations from $c^{\prime}$ can give a configuration that "matches" c. More formally, $c^{\prime}$ includes c if $\left(\left(6 v\left(v^{b}\right) \neq 6 v^{\prime}\left(v^{b}\right)\right) \Longrightarrow\left(6 v\left(v^{b}\right)=*\right)\right)$ for each $\mathrm{v}^{b} \in \mathrm{B}$ and there are injections $\kappa: \mathcal{T} \rightarrow \mathcal{T}^{\prime}$ and $\pi: \mathcal{P} \rightarrow \mathcal{P}^{\prime}$ such for each $t \in \mathcal{T}$ and $p \in \mathcal{P}$ :

1. $\left(\left(p c(t) \neq p c^{\prime}(\tau(t))\right) \Longrightarrow(p c(t)=*)\right)$,

2. either

- $p h a s e(t)(p)$ is undefined, or

- $p h a s e(t)(p)=(\mathrm{ph}$, val $)$ and phase $(\kappa(t))(\pi(p))=\left(\mathrm{ph}^{\prime}, v_{a l}^{\prime}\right)$ with $\left(\left(\mathrm{ph} \neq \mathrm{ph}^{\prime}\right) \Longrightarrow(\mathrm{ph}=*)\right)$ and either $\left(v a l=v a l^{\prime}=\mathrm{nreg}\right)$ or val $=($ wait, sig $)$ and val $^{\prime}=\left(\right.$ wait $^{\prime}$, sig $\left.^{\prime}\right)$ with $\left(\left(\right.\right.$ wait $\neq$ wait $\left.^{\prime}\right) \Longrightarrow$ $($ wait $=*))$ and $\left(\left(\operatorname{sig} \neq \operatorname{sig}^{\prime}\right) \Longrightarrow(\right.$ sig $\left.=*)\right)$.

Semantics and Reachability. Given a program $\operatorname{Pr}=(B, P, T s)$, the main task main ()$\{$ stmt $\}$ starts executing stmt from an initial configuration $\mathrm{c}_{\text {init }}=$ $\left(\mathcal{T}_{\text {init }}, \mathcal{P}_{\text {init }}, 6 v_{\text {init }}, p c_{\text {init }}\right.$, phase $\left.{ }_{\text {init }}\right)$ where $\mathcal{T}_{\text {init }}$ is a singleton, $\mathcal{P}_{\text {init }}$ is empty, $6 v_{\text {init }}$ sets all shared variables to false and $p c_{\text {init }}$ associates stmt to the unique task in $\mathcal{T}_{\text {init }}$.

We write $c \underset{\text { stmt }}{\stackrel{t}{\longrightarrow}} c^{\prime}$ to mean a task $t$ in $c$ can fire statement stmt and result in configuration $c^{\prime}$. See Figure 5.2 for a description of the operational semantics. We write $c \underset{\text { stmt }}{\longrightarrow} c^{\prime}$ if $c \underset{\text { stmt }}{\stackrel{t}{\longrightarrow}} c^{\prime}$ for some task $t$, and $c \rightarrow c^{\prime}$ if c $\underset{\text { stmt }}{\longrightarrow} c^{\prime}$ for some statement stmt. We also write $\stackrel{+}{\rightarrow}$ for the transitive closure of $\rightarrow$ and let $\stackrel{*}{\rightarrow}$ be the reflexive transitive closure of $\rightarrow$. Figure 5.3 identifies erroneous configurations.

We are interested in the reachability of sets of configurations (i.e., checking safety properties). We differentiate between two reachability problems depending on whether the target sets of configurations constrain the registration phases or not. The two problems are defined in the following.

Plain reachability. First, we define equivalent configurations. A configuration $\mathrm{c}=(\mathcal{T}, \mathcal{P}, 6 v, p c$, phase $)$ is equivalent to configuration $\mathrm{c}^{\prime}=$ $\left(\mathcal{T}^{\prime}, \mathcal{P}^{\prime}, 6 v^{\prime}, p c^{\prime}\right.$, phase $\left.{ }^{\prime}\right)$ if $6 v=6 v^{\prime}$ and there are bijections $\kappa: \mathcal{T} \rightarrow \mathcal{T}^{\prime}$ and $\pi: \mathcal{P} \rightarrow \mathcal{P}^{\prime}$ such that, for all $t \in \mathcal{T}, p \in \mathcal{P}$ and $\mathrm{ph} \in \mathrm{P} \cup\{-\}, p c(t)=p c^{\prime}(\kappa(t))$ and there are some integers $\left(k_{p}\right)_{p \in \mathcal{P}}$ such that phase $(t)(p)=(\mathrm{ph},(w, s))$ iff phase $^{\prime}(\kappa(t))(\pi(p))=\left(\mathrm{ph},\left(w+k_{p}, s+k_{p}\right)\right)$. We write $\mathrm{c} \sim \mathrm{c}^{\prime}$ to mean that $\mathrm{c}$ and $c^{\prime}$ are equivalent. Intuitively, equivalent configurations simulate each other. We can establish the following:

Lemma 4 (Equivalence). Assume two configurations $\mathrm{c}_{1}$ and $\mathrm{c}_{2}$. If $\mathrm{c}_{1} \rightarrow \mathrm{c}_{2}$ and $\mathrm{c}_{1}^{\prime} \sim \mathrm{c}_{1}$ then there is a configuration $\mathrm{c}_{2}^{\prime}$ such that $\mathrm{c}_{2}^{\prime} \sim \mathrm{c}_{2}$ and $\mathrm{c}_{1}^{\prime} \rightarrow \mathrm{c}_{2}^{\prime}$. 


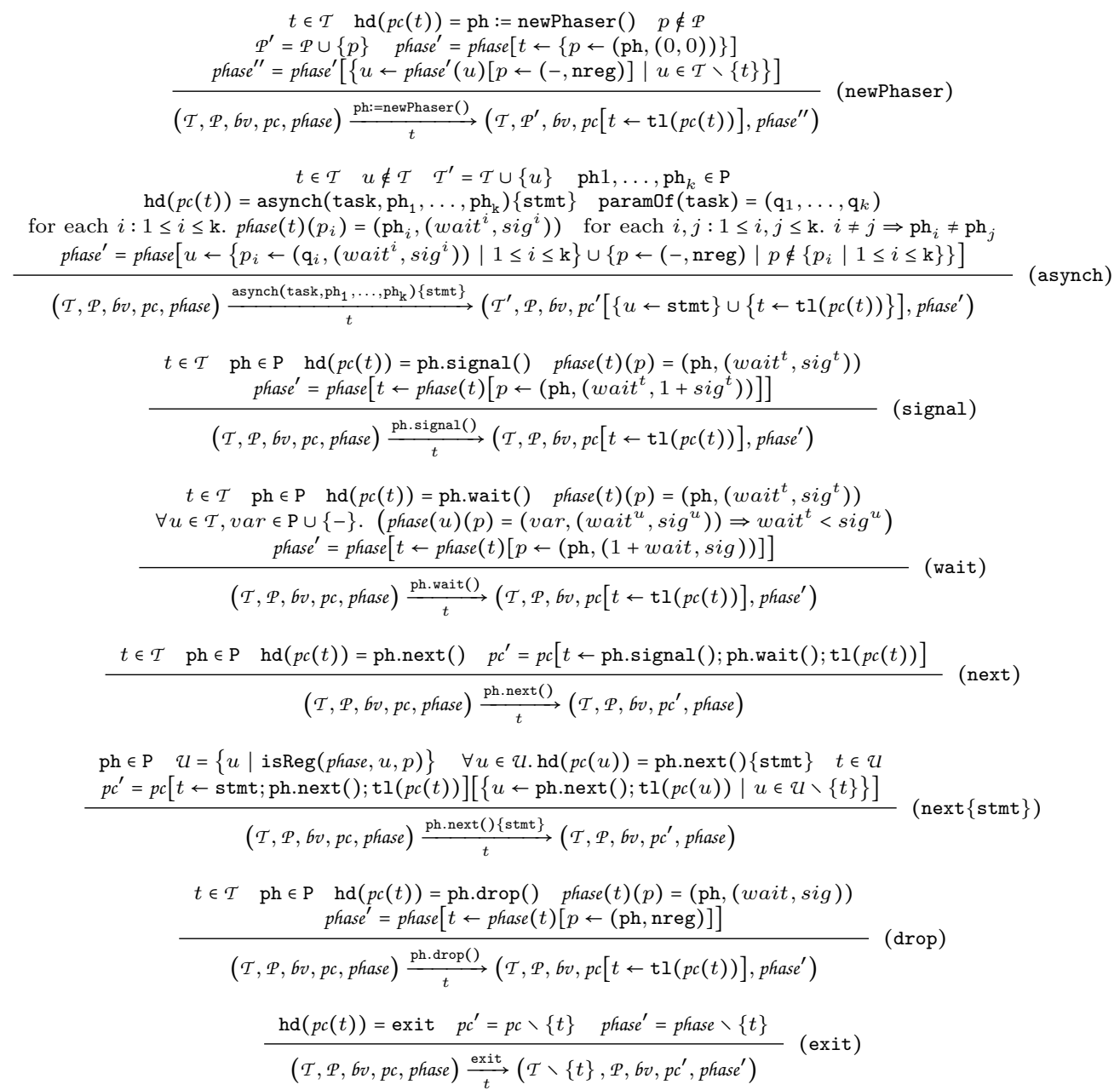

Figure 5.2: Operational semantics of phaser statements without errors. Each transition corresponds to a task $t \in \mathcal{T}$ executing a statement from a configuration $(\mathcal{T}, \mathcal{P}, 6 v, p c$, phase $)$. For instance, the drop transition corresponds to a task $t$ executing ph.drop() when registered with phaser $p \in \mathcal{P}$ (with phases (wait, sig)) and referring to it with variable ph. The result is the same configuration where task $t$ moves to its next statement without being registered with $p$ (albeit still referring to $p$ with $\mathrm{ph}$ ). 


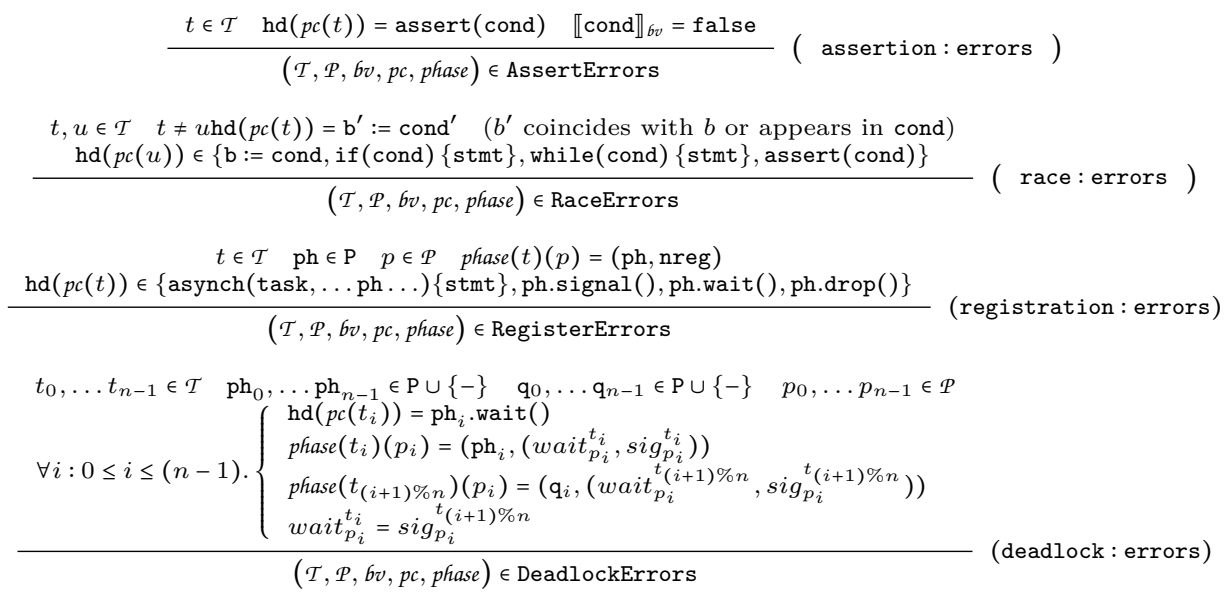

Figure 5.3: Definition of error configurations. Starting from $(\mathcal{T}, \mathcal{P}, 6 v, p c, p h a s e)$, error configurations are obtained when tasks execute the above statements under certain conditions. For instance, a deadlock is obtained if tasks in a subset $\left\{t_{0}, \ldots, t_{n-1}\right\} \subseteq \mathcal{T}$ form a cycle where each $t_{(i+1) \% n}$ blocks (with its signal phase $\operatorname{sig}_{p_{i}}^{t_{(i+1) \% n}}$ ) the wait being executed by $t_{i}$ on phaser $p_{i}$ (with wait phase wait $_{p_{i}}^{t_{i}}$ ).

Proof. According to the semantics in Figure 5.2, the gap between the wait and signal phases (not the absolute value of the phases) determines whether or not a phaser operation is enabled. This is because the phaser operations update the phases either by removing and adding phasers, or subtracting and adding a constant to them. The successors of equivalent configurations will therefore always be equivalent.

Observe that if the wait value of a task $t$ on a phaser $p$ is equal to the signal of a task $t^{\prime}$ on the same phaser $p$ in some configuration $\mathrm{c}$, then this is also the case, up to a renaming of the phasers and tasks, in all equivalent configurations. This is particularly relevant for defining deadlock configurations where several tasks are waiting for each other.

Given a program, a starting configuration, and a target partial configuration, the plain reachability problem asks whether a configuration that includes the target partial configuration is reachable. More formally, given a program $\operatorname{Pr}=(\mathrm{B}, \mathrm{P}, \mathrm{Ts})$, a configuration $\mathrm{c}_{\text {strt }}$, and a partial configuration $\mathrm{c}_{\text {trgt }}$, then reach $\left(\mathrm{Pr}, \mathrm{c}_{\text {strt }}, \mathrm{c}_{\text {trgt }}\right)$ iff $\mathrm{c}_{\text {strt }} \stackrel{*}{\rightarrow} \mathrm{c}_{1}$ for $\mathrm{c}_{1} \sim \mathrm{c}_{2}$ and $\mathrm{c}_{2}$ includes $\mathrm{c}_{\text {trgt }}$. We abuse notation and write $\mathrm{c}_{\text {strt }} \stackrel{*}{\rightarrow} \mathrm{C}$ to mean a configuration $\mathrm{c} \in \mathrm{C}$ that includes $\mathrm{c}_{\text {trgt }}$ is reachable from $\mathrm{c}_{\text {strt }}$. 
Definition 6 (Plain reachability). For a program $\mathrm{Pr}$, a configuration $\mathrm{c}_{\text {strt }}$, and a partial configuration $\mathrm{c}_{\text {trgt }}$, decide whether reach $\left(\operatorname{Pr}, \mathrm{c}_{\text {strt }}, \mathrm{c}_{\text {trgt }}\right)$ holds.

Control reachability. A partial configuration $\mathrm{c}=(\mathcal{T}, \mathcal{P}, 6 v, p c$, phase $)$ is said to be a control partial configuration if for all $t \in \mathcal{T}$ and $p \in \mathcal{P}$, either phase $(t)(p)$ is undefined or phase $(t)(p) \in(\mathrm{P} \cup\{-, *\} \times\{(*, *), \mathrm{nreg}\})$. Intuitively, control partial configurations do not constrain phase values. They are enough to characterize, for example, configurations where an assertion is violated (see Figure 5.3).

Definition 7 (Control reachability). For a program $\mathrm{Pr}$, a configuration $\mathrm{c}_{\text {strt }}$, and a control partial configuration $\mathrm{c}_{\text {trgt }}$, decide whether reach $\left(\operatorname{Pr}, \mathrm{c}_{\text {strt }}, \mathrm{c}_{\text {trgt }}\right)$ holds.

Observe that plain reachability is at least as hard to answer as control reachability since any control partial configuration is also a partial configuration. It turns out the control reachability problem is undecidable for programs that have arbitrarily many tasks and phasers, as stated by the theorem below.

Theorem 12. Control reachability is undecidable in general.

Proof. The proof is by reducing the reachability problem of 2-counter Minsky machines to the state reachability problem and using the Theorem 1 that was introduced in Chapter 3. Recall that the state reachability problem of a Minsky machine can be reduced to its reachability problem. State reachability of an arbitrary 2-counter Minsky machine is encoded as the control reachability problem of a phaser program (captured in Figure 5.5).

Assume a 2-counter Minsky machine $\mathrm{M}=\left(\mathcal{Q}, \mathcal{C}, \mathrm{T}, \mathcal{Q}_{\text {init }}, \mathrm{q}_{\text {trgt }}\right)$ with two counters $\mathcal{C}=\{x, y\}$. The phaser program has three tasks main, xTask and yTask. It uses shared Boolean variables to capture the state $\mathrm{q} \in \mathcal{Q}$ and pass information among different task instances. The phaser program builds two chains, one with xTask instances for the counter $x$, and one with yTask instances for the counter $y$. Each chain alternates a phaser and a task and encodes the values of its counter by the number of tasks in it. We explain the encoding for counter $x$. A similar encoding is used for counter $y$.

A chain for counter $x$ is the longest sequence of the form $t_{0}, p_{0}, t_{1}, p_{1} \ldots p_{m-1}, t_{m}$ where $t_{0}$ is running main and for all $k: 1 \leq k \leq m$ the tasks are running xTask. $t_{i}, p_{j}$ or $p_{j}, t_{i}$ appear in the chain iff $\operatorname{Reg}\left(t_{i}, p_{j}\right) . m$ is said to be the length of the chain. A sample configuration with two chains is illustrated in Figure 5.4. We show that $m$ models the value of counter $x$.

The idea is to have the phaser program simulate all counter machine transitions, i.e., increments, decrements, and tests for zero. Answering the state reachability of the counter machine amounts to checking whether from the initial configuration of the phaser program, $\mathrm{c}_{\text {init }}$, a configuration is reachable in which the Boolean variable $\mathrm{sF}$ that corresponds to the target machine state $\mathrm{q}_{F}$ is true. This can be captured with a control partial configuration. 


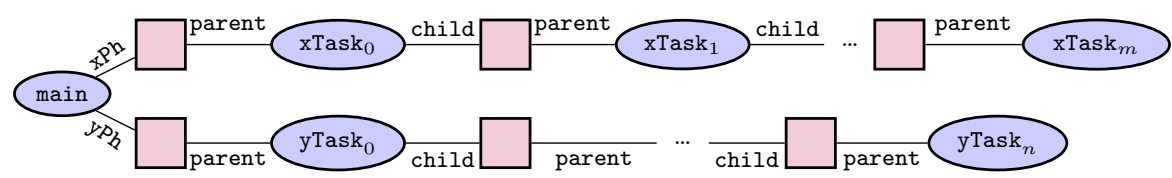

Figure 5.4: A sample configuration generated in the proof of Theorem 12 containing two chains of alternating tasks and phasers.

Increment. For incrementing counter $x$, main starts by executing signal and wait on $\mathrm{xPh}$ which has id $p_{0}$. If $m=0$, main will not block at line 12 . It creates $t_{1}$ and registers it with $p_{0}$ (line 14).

On the other hand, if $m>0$, we have the chain $t_{0}, p_{0}, t_{1}, p_{1} \ldots p_{m-1}, t_{m}$. In this case, $t_{k}$, for all $k: 0 \leq k \leq m-1$ executes signal and wait on the phaser $p_{k}$. Only $t_{m}$ is registered with the phaser $p_{m}$. Hence, only $t_{m}$ can pass the wait at line 61 . As a result, only $t_{m}$ sets temp (line 63 ), creates a new instance of xTask with id $t_{m+1}$, and registers it with $p_{m}$ (line 64). It will also issue signal on $p_{m}$ (line 65), hence release its parent who is blocked either at line 61 or line 12 . Now, every $t_{i}$ for $i: 0<i<m$ in the chain will release its parent by signaling at line 73 and will wait until all tasks other than main have done so. This is done by waiting at line 74 for temp to become false. At the end, main will reset temp and xInc and change the state.

Decrement. If $m=0$, decrementing $x$ is not enabled according to the semantics of the counter machines. In the encoded program, main will block at line 29. Otherwise, when $m>0$, we have the chain $t_{0}, p_{0}, t_{1}, p_{1} \ldots p_{m-1}, t_{m}$. In this case, $t_{k}$, for all $k: 0 \leq k \leq m-1$ executes signal and wait on the phaser $p_{k}$. Only $t_{m}$ is registered with the phaser $p_{m}$. Hence, only $t_{m}$ can pass the wait on line 61. Hence, only $t_{m}$ will set temp (line 69) and exit (line 70). Now, every $t_{i}$ for $i: 0<i<m$ in the chain will release its parent by signaling at line 73 and will wait until all tasks other than main have done so. This is done by waiting at line 74 for temp to become false. At the end, main will reset temp and $\mathrm{xDec}$ and change the state.

Test for zero is encoded as a signal followed by a wait on xPh (lines 37 and 38). If some $t_{m}$ exists for $m>0$ (that is, $x>0$ ), main will block at line 38. Otherwise, it will only change the state accordingly.

The above encoding ensures that the lengths of the xTask and yTask chains capture the values of the counters $x$ and $y$, respectively. As a result, from the initial configuration $\mathrm{c}_{\text {init }}$, the phaser program will reach a configuration in which $\mathrm{sF}=$ true iff the counter machine reaches a configuration with the target state $\mathbf{q}_{F}$. 
We have demonstrated that the control reachability problem is in general undecidable. In the next section, we introduce our symbolic representation that will be used in the verification procedure.

\subsection{A Gap-based Symbolic Representation}

The symbolic representation we propose builds on the following intuitions. First, observe that the language semantics impose, for each phaser, the invariant that signal values are always greater than or equal to wait values. We can, therefore, assume this fact in our symbolic representation. Also, our reachability problems from Section 5.3 are defined in terms of reachability of equivalence classes, not of individual configurations. This is because configurations that violate the considered properties (see Figure 5.3) are not defined in terms of concrete phase values but rather in terms of relations among them (as well as the registration status, control sequences, and variable values). Finally, we observe that if a wait is enabled with smaller gaps on a given phaser, then it will be enabled with larger ones. We, therefore, propose to track the gaps of the differences between signal and wait values with respect to an existentially quantified level (per phaser) that lies between wait and signal values of all registered tasks (with the phaser that is being considered).

We formally define our symbolic representation and describe a corresponding entailment relation. We also establish a desirable property (namely that of being a well-quasi ordering) on some classes of representations. This property is crucial for the decidability of specific reachability problems (see. Section $5.5)$.

Named gaps. A named gap is associated to a task-phaser pair. It consists of a tuple $(\mathrm{ph}, \mathrm{val})$ in $\mathbb{G}=\left(\mathrm{P} \cup\{-, *\} \times\left(\left(\mathbb{N}^{4} \cup\left(\mathbb{N}^{2} \times\{\infty\}^{2}\right)\right) \cup\{\right.\right.$ nreg $\left.\left.\}\right)\right)$. Like for partial configurations in Section 5.3, ph $\in \mathrm{P} \cup\{-, *\}$ constrains variable values. The val value describes task registration to the phaser. If not registered, val is nreg. Otherwise, val is a 4-tuple (lw, ls, uw, us). This tuple intuitively captures, together with some level lev common to all tasks registered with the considered phaser, all concrete wait and signal values (wait,sig) satisfying $I_{w} \leq($ lev - wait $) \leq$ uw and $I_{s} \leq(s i g-$ lev $) \leq$ us. A named gap $(\mathrm{ph},(1 \mathrm{w}, 1 \mathrm{~s}, \mathrm{uw}, \mathrm{us}))$ is said to be free if uw $=\mathrm{us}=\infty$. It is said to be $B$ gap-bounded, for $B \in \mathbb{N}$, if both uw $\leq B$ and us $\leq B$ hold. (ph,nreg) is $B$-gap-bounded for any $B \in \mathbb{N}$. A set $\mathcal{G} \subseteq \mathbb{G}$ is said to be free (respectively $B$-gap-bounded) if all its named gaps are free (respectively $B$-gap-bounded). The set $\mathcal{G}$ is said to be $B$-good if each one of its named gaps is either free or $B$-gap-bounded. Finally, $\mathcal{G}$ is said to be good if it is $B$-good for some $B \in \mathbb{N}$. Given a set $\mathcal{G}$ of named gaps, we define the partial order $\unlhd$ on $\mathcal{G}$, and write $(\mathrm{ph}, \mathrm{val}) \unlhd\left(\mathrm{ph}^{\prime}, \mathrm{val}^{\prime}\right)$, to mean (i) $\left(\mathrm{ph} \neq \mathrm{ph}^{\prime} \Rightarrow \mathrm{ph}=*\right)$, and (ii) $(\mathrm{val}=\mathrm{nreg}) \Longleftrightarrow\left(\mathrm{val}^{\prime}=\mathrm{nreg}\right)$, and (iii) if val $=(1 \mathrm{w}, 1 \mathrm{~s}, \mathrm{uw}, \mathrm{us})$ and 
val $^{\prime}=\left(1 w^{\prime}, l s^{\prime}, u^{\prime}, u^{\prime}\right)$ then $l w \leq l w^{\prime}, l s \leq l s^{\prime}, u_{w}^{\prime} \leq$ uw and $u s^{\prime} \leq$ us. Intuitively, named gaps are used in the definition of constraints in order to capture relations (i.e., reference, registration, and possible phases) of tasks and phasers. The partial order $(\mathrm{ph}, \mathrm{val}) \unlhd\left(\mathrm{ph}^{\prime}, \mathrm{val}^{\prime}\right)$ ensures the relations allowed by $\left(\mathrm{ph}^{\prime}, \mathrm{val}^{\prime}\right)$ are also allowed by (ph, val).

Constraints. A constraint $\varphi$ of a phaser program $\operatorname{Pr}=(B, P, T s)$ is a tuple $(\mathcal{T}, \mathcal{P}, 6 v, p c, g a p$, egap $)$ that denotes a possibly infinite set of configurations. Intuitively, $\mathcal{T}$ and $\mathcal{P}$ respectively represent a minimal set of tasks and phasers that are required in any configuration denoted by the constraint. We only consider constraints that have at most one instance of the task main. In addition:

- $6 v: \mathrm{B} \rightarrow \mathbb{B} \cup\{*\}$ and $p c: \mathcal{T} \rightarrow \mathrm{S} \cup\{*\}$ respectively represent, like for partial configurations, a valuation of the Boolean variables and a mapping of tasks to their control sequences.

- gap $: \mathcal{T} \rightarrow \operatorname{Fn}(\mathcal{P}, \mathbb{G})$ constrains relations between $\mathcal{T}$-tasks and $\mathcal{P}$-phasers by associating to each task $t$ a mapping gap $(t)$ that defines for each phaser $p$ a named gap ( $\mathrm{ph}, \mathrm{val}) \in \mathbb{G}$ capturing the relation of $t$ and $p$.

- egap $: \mathcal{P} \rightarrow \mathbb{N}^{2}$ associates lower bounds (ew, es) on gaps of tasks that are registered with $\mathcal{P}$-phasers but which are not explicitly captured by $\mathcal{T}$. This is described further in the denotations of a constraint below.

We write isReg $(t, p, g a p)$ to mean a task $t$ is registered with a phaser $p$, i.e., $\operatorname{gap}(t)(p) \notin(\mathrm{P} \cup\{-, *\} \times\{\mathrm{nreg}\})$. A constraint $\varphi$ is said to be free (respectively $B$-gap-bounded or $B$-good) if the set $\mathcal{G}=\{\operatorname{gap}(t)(p) \mid t \in \mathcal{T}, p \in \mathcal{P}\}$ is free (respectively, $B$-gap-bounded or $B$-good). The dimension of a constraint is the number of its phasers (i.e., $|\mathcal{P}|$ ). A set of constraints is said to be $K$-dimension-bounded if there is natural number $K$ in $\mathbb{N}$ that is larger than the dimension of any of its elements. A set of constraints $\Phi$ is said to be free, $B$-gap-bounded, or $B$-good if each of its constraints is.

Denotations. For a constraint $\varphi=\left(\mathcal{T}_{\varphi}, \mathcal{P}_{\varphi}, b v_{\varphi}, p c_{\varphi}, \operatorname{gap}_{\varphi}, \operatorname{egap}_{\varphi}\right)$ and a configuration $c=\left(\mathcal{T}_{c}, \mathcal{P}_{c}, b v_{c}, p c_{c}\right.$, phase $\left.{ }_{c}\right)$, we write $c \vDash \varphi$ (or $c \in[[\varphi]]$ ) to mean $\varphi$ denotes c. Intuitively, the configuration $\mathrm{c}$ should have at least as many tasks (captured by a surjection $\kappa$ from a subset $\mathcal{T}_{\mathrm{c}}^{1}$ of $\mathcal{T}_{\mathrm{c}}$ to $\mathcal{T}_{\varphi}$ ) and phasers (captured by a bijection $\pi$ from a subset $\mathcal{P}_{\mathrm{c}}^{1}$ of $\mathcal{P}_{\mathrm{c}}$ to $\mathcal{P}_{\varphi}$ ). Constraints on the tasks and phasers in $\mathcal{T}_{c}^{1}$ and $\mathcal{P}_{c}^{1}$ ensure target configurations are reachable. Additional constraints on the tasks in $\mathcal{T}_{c}^{2}=\mathcal{T}_{c} \backslash \mathcal{T}_{c}^{1}$ ensure this reachability is not blocked by tasks not captured by $\mathcal{T}_{\varphi}$. More formally:

1. for each $\mathrm{v}^{b} \in \mathrm{B},\left(6 v_{\varphi}\left(\mathrm{v}^{b}\right) \neq b v_{\mathrm{c}}\left(\mathrm{v}^{b}\right)\right) \Longrightarrow\left(6 v_{\varphi}\left(\mathrm{v}^{b}\right)=*\right)$, and

2. $\mathcal{T}_{\mathrm{c}}$ and $\mathcal{P}_{\mathrm{c}}$ can be written as $\mathcal{T}_{\mathrm{c}}=\mathcal{T}_{\mathrm{c}}^{1} \uplus \mathcal{T}_{\mathrm{c}}^{2}$ and $\mathcal{P}_{\mathrm{c}}=\mathcal{P}_{\mathrm{c}}^{1} \uplus \mathcal{P}_{\mathrm{c}}^{2}$, with 
3. $\kappa: \mathcal{T}_{\mathrm{c}}^{1} \rightarrow \mathcal{T}_{\varphi}$ is a surjection and $\pi: \mathcal{P}_{\mathrm{c}}^{1} \rightarrow \mathcal{P}_{\varphi}$ is a bijection, and

4. for $t_{\mathrm{c}} \in \mathcal{T}_{\mathrm{c}}^{1}$ with $t_{\varphi}=\kappa\left(t_{\mathrm{c}}\right),\left(p c_{\varphi}\left(t_{\varphi}\right) \neq p c_{\mathrm{c}}\left(t_{\mathrm{c}}\right)\right) \Longrightarrow\left(p c_{\varphi}\left(t_{\varphi}\right)=*\right)$, and

5. for each $p_{\varphi}=\pi\left(p_{c}\right)$, there is a natural level lev $\geq 0$ such that:

a) if $t_{\mathrm{c}} \in \mathcal{T}_{\mathrm{c}}^{1}$ with $t_{\varphi}=\kappa\left(t_{\mathrm{c}}\right)$, phase pro $\left._{\mathrm{c}}\right)\left(p_{\mathrm{c}}\right)=\left(\mathrm{ph}_{\mathrm{c}}, \mathrm{val}_{\mathrm{c}}\right)$ and $\operatorname{gap}_{\varphi}\left(t_{\varphi}\right)\left(p_{\varphi}\right)=\left(\mathrm{ph}_{\varphi}, \operatorname{val}_{\varphi}\right)$, then it is the case that:

i. $\left(\mathrm{ph}_{\mathrm{c}} \neq \mathrm{ph}_{\varphi}\right) \Longrightarrow\left(\mathrm{ph}_{\varphi}=*\right)$, and

ii. $\left(\mathrm{val}_{\mathrm{c}}=\mathrm{nreg}\right) \Longleftrightarrow\left(\operatorname{val}_{\varphi}=\mathrm{nreg}\right)$, and

iii. if $\left(\operatorname{val}_{\mathrm{c}}=(\right.$ wait, sig $\left.)\right)$ and $\left(\operatorname{val}_{\varphi}=(1 \mathrm{w}, 1 \mathrm{~s}, \mathrm{uw}, \mathrm{us})\right)$ then $1 \mathrm{w} \leq$ lev-wait $\leq$ uw and $1 \mathrm{~s} \leq$ sig - lev $\leq$ us.

b) if $t_{\mathrm{c}} \in \mathcal{T}_{\mathrm{c}}^{2}$, then for each $p_{\varphi}=\pi\left(p_{\mathrm{c}}\right)$ with phase ${ }_{\mathrm{c}}\left(t_{\mathrm{c}}\right)\left(p_{\mathrm{c}}\right)=$ $\left(\mathrm{ph}_{\mathrm{c}},(\right.$ wait, sig $\left.)\right)$ and $\operatorname{egap}\left(p_{\varphi}\right)=(\mathrm{ew}, \mathrm{es})$, we have: $(\mathrm{es} \leq \operatorname{sig}-\mathrm{lev})$ and $(\mathrm{ew} \leq$ lev - wait $)$

We say in this case that $\kappa$ and $\pi$ witness the denotation of c by $\varphi$. Intuitively, for each phaser, the bounds given by gap constrain the values of the phases belonging to tasks captured by $\mathcal{T}_{\varphi}$ (i.e., those in $\mathcal{T}_{c}^{1}$ ) and registered with the given phaser. This is done with respect to some non-negative level, one per phaser. The same level is used to constrain phases of tasks registered with the phaser but not captured by $\mathcal{T}_{\varphi}$ (i.e., those in $\mathcal{T}_{c}^{2}$ ). For these tasks, lower bounds are enough as we only want to ensure that they do not block executions to target sets of configurations. We write $[\varphi \varphi]$ for $\{c \mid c \vDash \varphi\}$.

Entailment. We write $\varphi_{a} \sqsubseteq \varphi_{b}$ to mean $\varphi_{a}=\left(\mathcal{T}_{a}, \mathcal{P}_{a}, b v_{a}, p c_{a}, g a p_{a}\right.$, egap $\left.a\right)$ is entailed by $\varphi_{b}=\left(\mathcal{T}_{b}, \mathcal{P}_{b}, 6 v_{b}, p c_{b}, g a p_{b}, e g a p_{b}\right)$. This will ensure that configurations denoted by $\varphi_{b}$ are also denoted by $\varphi_{a}$. Intuitively, $\varphi_{b}$ should have at least as many tasks (captured by a surjection $\kappa$ from a subset $\mathcal{T}_{b}^{1}$ of $\mathcal{T}_{b}$ to $\mathcal{T}_{a}$ ) and phasers (captured by a bijection $\pi$ from a subset $\mathcal{P}_{b}^{1}$ of $\mathcal{P}_{b}$ to $\mathcal{P}_{a}$ ). Conditions on tasks and phasers in $\mathcal{T}_{b}^{1}$ and $\mathcal{P}_{b}^{1}$ ensure the conditions in $\varphi_{a}$ are met. Additional conditions on the tasks in $\mathcal{T}_{b}^{2}=\mathcal{T}_{b} \backslash \mathcal{T}_{b}^{1}$ ensure at least the egap $_{a}$ conditions in $\varphi_{a}$ are met. More formally:

1. $\left(6 v_{a}\left(\mathrm{v}^{b}\right) \neq 6 v_{b}\left(\mathrm{v}^{b}\right)\right) \Longrightarrow\left(6 v_{a}\left(\mathrm{v}^{b}\right)=*\right)$, for each $\mathrm{v}^{b} \in \mathrm{B}$ and

2. $\mathcal{T}_{b}$ and $\mathcal{P}_{b}$ can be written as $\mathcal{T}_{b}=\mathcal{T}_{b}^{1} \uplus \mathcal{T}_{b}^{2}$ and $\mathcal{P}_{b}=\mathcal{P}_{b}^{1} \uplus \mathcal{P}_{b}^{2}$ with

3. $\kappa: \mathcal{T}_{b}^{1} \rightarrow \mathcal{T}_{a}$ is a surjection and $\pi: \mathcal{P}_{b}^{1} \rightarrow \mathcal{P}_{a}$ is a bijection, and

4. $\left(p c_{b}\left(t_{b}\right) \neq p c_{a}\left(t_{a}\right)\right) \Longrightarrow\left(p c_{b}\left(t_{b}\right)=*\right)$ for each $t_{b} \in \mathcal{T}_{b}^{1}$ with $t_{a}=\kappa\left(t_{b}\right)$, and

5. for each phaser $p_{a}=\pi\left(p_{b}\right)$ in $\mathcal{P}_{a}$ :

a) if $\operatorname{egap}_{a}\left(p_{a}\right)=\left(\mathrm{ew}_{a}, \mathrm{es}_{a}\right)$ and $\operatorname{egap}_{b}\left(p_{b}\right)=\left(\mathrm{ew}_{b}, \mathrm{es}_{b}\right)$ then $\mathrm{ew}_{a} \leq \mathrm{ew}_{b}$ and $\mathrm{es}_{a} \leq \mathrm{es}_{b}$ 
b) for each $t_{b} \in \mathcal{T}_{b}^{1}$ with $t_{a}=\kappa\left(t_{b}\right)$ and gap $\left(t_{a}\right)\left(p_{a}\right)=\left(\mathrm{ph}_{a}, \operatorname{val}_{a}\right)$, and $\operatorname{gap}_{b}\left(t_{b}\right)\left(p_{b}\right)=\left(\mathrm{ph}_{b}, \mathrm{val}_{b}\right)$, it is the case that:

i. $\left(\mathrm{ph}_{b} \neq \mathrm{ph}_{a}\right) \Longrightarrow\left(\mathrm{ph}_{a}=*\right)$, and

ii. $\left(\mathrm{val}_{b}=\mathrm{nreg}\right) \Longleftrightarrow\left(\mathrm{val}_{a}=\mathrm{nreg}\right)$, and

iii. if $\mathrm{val}_{a}=\left(1 \mathrm{w}_{a}, 1 \mathrm{~s}_{a}, \mathrm{uw}_{a}, \mathrm{us}_{a}\right)$ and $\mathrm{val}_{b}=\left(1 \mathrm{w}_{b}, 1 \mathrm{~s}_{b}, \mathrm{uw}_{b}, \mathrm{us}_{b}\right)$, then $\left(1 \mathrm{w}_{a} \leq 1 \mathrm{w}_{b}\right),\left(1 \mathbf{s}_{a} \leq 1 \mathbf{s}_{b}\right),\left(\mathrm{uw}_{b} \leq \mathrm{uw}_{a}\right)$ and $\left(\mathrm{us}_{b} \leq \mathrm{us}_{a}\right)$.

c) for each $t_{b} \in \mathcal{T}_{b}^{2}$ with $\operatorname{gap}_{b}\left(t_{b}\right)\left(p_{b}\right)=\left(\mathrm{ph},\left(1 \mathrm{w}_{a}, 1 \mathbf{s}_{a}, \mathrm{uw}_{a}, \mathrm{us}_{a}\right)\right)$, with $\operatorname{egap}_{a}\left(p_{a}\right)=\left(\mathrm{ew}_{a}, \mathrm{es}_{a}\right)$, both $\left(\mathrm{ew}_{a} \leq 1 \mathrm{w}_{b}\right)$ and $\left(\mathrm{es} a \leq 1 \mathrm{~s}_{b}\right)$ hold.

We say in this case that $\kappa$ and $\pi$ witness the entailment of $\varphi_{a}$ by $\varphi_{b}$.

Lemma 5 (Constraint entailment). $\varphi_{a} \subseteq \varphi_{b}$ implies $\left[\left[\varphi_{b}\right] \subseteq\left[\left[\varphi_{a}\right]\right]\right.$

Proof. Assume a configuration $\mathrm{c}=\left(\mathcal{T}_{\mathrm{c}}, \mathcal{P}_{\mathrm{c}}, 6 v_{\mathrm{c}}, p c_{\mathrm{c}}\right.$, phase $\left.{ }_{\mathrm{c}}\right)$ is denoted by $\varphi_{b}=$ $\left(\mathcal{T}_{b}, \mathcal{P}_{b}, 6 v_{b}, p c_{b}, \operatorname{gap}_{b}, \operatorname{egap}_{a}\right)$ with $\varphi_{a} \sqsubseteq \varphi_{b}$ and $\varphi_{a}=\left(\mathcal{T}_{a}, \mathcal{P}_{a}, 6 v_{a}, p c_{a}, g a p_{a}\right.$, egap $\left._{a}\right)$. We show $\mathrm{c}$ is also denoted by $\varphi_{a}$.

By assumption, we can write $\mathcal{T}_{\mathrm{c}}$ as a partition $\mathcal{T}_{\mathrm{c}}^{m} \uplus \mathcal{T}_{\mathrm{c}}^{e}$ and $\mathcal{P}_{\mathrm{c}}$ as a partition $\mathcal{P}_{\mathrm{c}}^{m} \uplus \mathcal{P}_{\mathrm{c}}^{e}$ such that a surjection $\kappa_{\mathrm{c}}: \mathcal{T}_{\mathrm{c}}^{m} \rightarrow \mathcal{T}_{b}$ and a bijection $\pi_{\mathrm{c}}: \mathcal{P}_{\mathrm{c}}^{m} \rightarrow \mathcal{P}_{b}$ witness the denotation of c by $\varphi_{b}$. Also, we can write $\mathcal{T}_{b}$ as a partition $\mathcal{T}_{b}^{m} \uplus \mathcal{T}_{b}^{e}$ and $\mathcal{P}_{b}$ as a partition $\mathcal{P}_{b}^{m} \uplus \mathcal{P}_{b}^{e}$ such that a surjection $\kappa_{\varphi}: \mathcal{T}_{b}^{m} \rightarrow \mathcal{T}_{a}$ and a bijection $\pi_{\varphi}: \mathcal{P}_{b}^{m} \rightarrow \mathcal{P}_{a}$ witness the entailment of $\varphi_{a}$ by $\varphi_{b}$. Let us write $\mathcal{T}_{\mathrm{c}}^{m}$ as the partition $\mathcal{T}_{\mathrm{c}}^{m, m} \uplus \mathcal{T}_{\mathrm{c}}^{m, e}$ where $\mathcal{T}_{\mathrm{c}}^{m, m}=\kappa_{\mathrm{c}}^{-1}\left(\mathcal{T}_{b}^{m}\right)$ and $\mathcal{P}_{\mathrm{c}}^{m}$ as the partition $\mathcal{P}_{\mathrm{c}}^{m, m} \uplus \mathcal{P}_{\mathrm{c}}^{m, e}$ where $\mathcal{P}_{\mathrm{c}}^{m, m}=\pi_{\mathrm{c}}^{-1}\left(\mathcal{P}_{b}^{m}\right)$. We define $\kappa$ to be the restriction of $\kappa_{\varphi} \circ \kappa_{\mathrm{c}}$ to $\mathcal{T}_{\mathrm{c}}^{m, m}$, i.e., $\kappa: \mathcal{T}_{\mathrm{c}}^{m, m} \rightarrow \mathcal{T}_{a}$ with $\kappa(t)=\kappa_{\varphi}\left(\kappa_{\mathrm{c}}(t)\right)$ for each $t$ in $\mathcal{T}_{c}^{m, m}$. Observe that $\kappa$ is a well-defined surjection. In addition, we write $\pi$ to mean the restriction of $\pi_{\varphi} \circ \pi_{\mathrm{c}}$ to $\mathcal{P}_{\mathrm{c}}^{m, m}$. Observe that $\pi$ is a well-defined bijection. We show that $\kappa$ and $\pi$ witness the denotation of c by $\varphi_{a}$.

1. we have that, for each $\mathrm{v}^{b} \in \mathrm{B}$, both $\left(6 v_{b}\left(\mathrm{v}^{b}\right) \neq 6 v_{\mathrm{c}}\left(\mathrm{v}^{b}\right)\right) \Longrightarrow\left(6 v_{b}\left(\mathrm{v}^{b}\right)=\right.$ $*)$ and $\left(6 v_{a}\left(\mathrm{v}^{b}\right) \neq b v_{b}\left(\mathrm{v}^{b}\right)\right) \Longrightarrow\left(6 v_{a}\left(\mathrm{v}^{b}\right)=*\right)$ hold. Hence, $\left(6 v_{a}\left(\mathrm{v}^{b}\right) \neq\right.$ $\left.6 v_{\mathrm{c}}\left(\mathrm{v}^{b}\right)\right) \Longrightarrow\left(6 v_{a}\left(\mathrm{v}^{b}\right)=*\right)$ also holds.

2. $\mathcal{T}_{\mathrm{c}}=\mathcal{T}_{\mathrm{c}}^{m, m} \uplus\left(\mathcal{T}_{\mathrm{c}}^{m, e} \uplus \mathcal{T}_{\mathrm{c}}^{e}\right)$ and $\mathcal{P}_{\mathrm{c}}=\mathcal{P}_{\mathrm{c}}^{m, m} \uplus\left(\mathcal{P}_{\mathrm{c}}^{m, e} \uplus \mathcal{P}_{\mathrm{c}}^{e}\right)$, with

3. $\kappa: \mathcal{T}_{\mathrm{c}}^{m, m} \rightarrow \mathcal{T}_{a}$ is a surjection and $\pi: \mathcal{P}_{\mathrm{c}}^{m, m} \rightarrow \mathcal{P}_{a}$ is a bijection, such that

4. we have that $\left(p c_{b}\left(t_{b}\right) \neq p c_{c}\left(t_{c}\right)\right) \Longrightarrow\left(p c_{b}\left(t_{b}\right)=*\right)$ for each $t_{c} \in \mathcal{T}_{c}^{m}$ with $t_{b}=\kappa_{c}\left(t_{c}\right)$ in $\mathcal{T}_{b}$, and $\left(p c_{a}\left(t_{a}\right) \neq p c_{b}\left(t_{b}\right)\right) \Longrightarrow\left(p c_{a}\left(t_{a}\right)=*\right)$ for each $t_{b} \in \mathcal{T}_{b}^{m}$ with $t_{a}=\kappa_{\varphi}\left(t_{b}\right)$ in $\mathcal{T}_{a}$. Since $\mathcal{T}_{b}^{m, m} \subseteq \mathcal{T}_{b}^{m}$ and the surjection $\kappa: \mathcal{T}_{c}^{m, m} \rightarrow \mathcal{T}_{b}$ is the restriction of $\kappa_{\varphi} \circ \kappa_{\mathrm{c}}$ to $\mathcal{T}_{\mathrm{c}}^{m, m}$, we deduce: $\left(p c_{a}\left(t_{a}\right) \neq p c_{c}\left(t_{c}\right)\right) \Longrightarrow\left(p c_{a}\left(t_{a}\right)=*\right)$ for each $t_{\mathrm{c}} \in \mathcal{T}_{\mathrm{c}}^{m, m}$ with $t_{a}=\kappa\left(t_{\mathrm{c}}\right)$ in $\mathcal{T}_{a}$.

5. for each $p_{a}=\pi_{\varphi}\left(p_{b}\right)=\pi_{\varphi}\left(\pi_{\mathrm{c}}\left(p_{\mathrm{c}}\right)\right)=\pi\left(p_{\mathrm{c}}\right)$, there is a lev : $0 \leq$ lev s.t.: 
a) if $t_{\mathrm{c}} \in \mathcal{T}_{\mathrm{c}}^{m, m}$ with $t_{a}=\kappa_{\varphi}\left(\kappa_{\mathrm{c}}\left(t_{\mathrm{c}}\right)\right)$ with phase ${ }_{\mathrm{c}}\left(t_{\mathrm{c}}\right)\left(p_{\mathrm{c}}\right)=\left(\mathrm{ph}_{\mathrm{c}}, \mathrm{val}_{\mathrm{c}}\right)$, $\operatorname{gap}_{b}\left(t_{b}\right)\left(p_{b}\right)=\left(\mathrm{ph}_{b}, \operatorname{val}_{b}\right)$ and $\operatorname{gap}_{a}\left(t_{a}\right)\left(p_{a}\right)=\left(\mathrm{ph}_{a}, \mathrm{val}_{a}\right)$, then:

i. we have $\left(\mathrm{ph}_{a} \neq \mathrm{ph}_{b}\right) \Longrightarrow\left(\mathrm{ph}_{a}=*\right)$ and $\left(\mathrm{ph}_{b} \neq \mathrm{ph}_{\mathrm{c}}\right) \Longrightarrow$ $\left(\mathrm{ph}_{b}=*\right)$. Hence, $\left(\mathrm{ph}_{a} \neq \mathrm{ph}_{\mathrm{c}}\right) \Longrightarrow\left(\mathrm{ph}_{a}=*\right)$.

ii. $\left(\mathrm{val}_{\mathrm{c}}=\mathrm{nreg}\right) \Longleftrightarrow\left(\mathrm{val}_{b}=\mathrm{nreg}\right) \Longleftrightarrow\left(\mathrm{val}_{a}=\mathrm{nreg}\right)$, and

iii. if $\left(\mathrm{val}_{\mathrm{c}}=(\right.$ wait, sig $\left.)\right)$ and $\left(\mathrm{val}_{b}=\left(1 \mathrm{w}_{b}, \mathrm{ls}_{b}, \mathrm{uw}_{b}, \mathrm{us}_{b}\right)\right)$ and $\left(\operatorname{val}_{a}=\left(1 \mathrm{w}_{a}, 1 \mathrm{~s}_{a}, \mathrm{uw}_{a}, \mathrm{us}_{a}\right)\right)$, then $1 \mathrm{w}_{a} \leq 1 \mathrm{w}_{b} \leq$ lev - wait $\leq$ $\mathrm{uw}_{b} \leq \mathrm{uw}_{a}$ and $\mathbf{l} \mathrm{s}_{a} \leq 1 \mathrm{~s}_{b} \leq s i g-l e v \leq \mathrm{us}_{b} \leq \mathrm{u} \mathbf{s}_{a}$.

b) if $t_{\mathrm{c}} \in \mathcal{T}_{\mathrm{c}}^{m, e}$ with $t_{b}=\kappa_{\mathrm{c}}\left(t_{\mathrm{c}}\right)$ with phase $\left(t_{\mathrm{c}}\right)\left(p_{\mathrm{c}}\right)=\left(\mathrm{ph}_{\mathrm{c}}, \mathrm{val}_{\mathrm{c}}\right)$, $\operatorname{gap}_{b}\left(t_{b}\right)\left(p_{b}\right)=\left(\mathrm{ph}_{b}, \mathrm{val}_{b}\right)$ and $\operatorname{egap}_{a}\left(p_{a}\right)=\left(\mathrm{ew}_{a}, \mathrm{es}_{a}\right)$, then:

i. if $\left(\operatorname{val}_{c}=(\right.$ wait, sig $\left.)\right)$ and $\left(\operatorname{val}_{b}=\left(1 \mathrm{w}_{b}, 1 \mathbf{s}_{b}, \mathrm{uw}_{b}, \mathrm{us}_{b}\right)\right)$ and $\operatorname{egap}_{a}\left(p_{a}\right)=\left(\mathrm{ew}_{a}, \mathrm{es}_{a}\right)$ then $\mathrm{ew}_{a} \leq 1 \mathrm{w}_{b} \leq$ lev - wait and es $\mathrm{e}_{a} \leq$ $1 \mathrm{~s}_{b} \leq$ sig - lev.

c) if $t_{\mathrm{c}} \in \mathcal{T}_{\mathrm{c}}^{e}$ with phase ${ }_{\mathrm{c}}\left(t_{\mathrm{c}}\right)\left(p_{\mathrm{c}}\right)=\left(\mathrm{ph}_{\mathrm{c}},(\right.$ wait, sig $\left.)\right)$ and $\operatorname{egap}_{b}\left(p_{b}\right)=$ $\left(\mathrm{ew}_{b}, \mathrm{es}_{b}\right)$ and $\operatorname{egap}_{a}\left(p_{a}\right)=\left(\mathrm{ew} a, \mathrm{es}_{a}\right)$, then: es $\mathrm{es}_{a} \leq \mathrm{es}_{b} \leq \mathrm{sig}-\mathrm{lev}$ and $\mathrm{ew}_{a} \leq \mathrm{ew}_{b} \leq$ lev - wait

The remaining part of this section aims to establish the following theorem:

Theorem 13. $(\Phi$, ㄷ) is $\mathcal{W} \mathcal{Q O}$ if $\Phi$ is $K$-dimension-bounded and B-good for some pre-defined $K, B \in \mathbb{N}$.

The idea is to propose an encoding for each constraint $\varphi=$ $(\mathcal{T}, \mathcal{P}, 6 v, p c$, gap, egap $)$ with respect to some arbitrary total orders $<_{\mathcal{P}}$ and $<_{\mathcal{T}}$. We write enc $\left(\varphi,<_{\mathcal{T}},<_{\mathcal{P}}\right)$ for the encoding of $\varphi$. We also define an entailment relation $\leq_{e}$ on the encodings. Then, we show in Lemma 10 that $\operatorname{enc}\left(\varphi,<_{\mathcal{T}},<_{\mathcal{P}}\right) \leq_{e} \operatorname{enc}\left(\varphi^{\prime},<_{\mathcal{T}^{\prime}},<_{\mathcal{P}^{\prime}}\right)$ implies $\varphi \sqsubseteq \varphi^{\prime}$. Finally, we show in Lemma 11 that $\leq_{e}$ is $\mathcal{W} \mathcal{Q O}$ if the encoded $K$-dimension-bounded constraints are $B$-good for some pre-defined $K, B \in \mathbb{N}$. We start with the named gaps.

Lemma 6. If $\mathcal{G}$ is a good set of named gaps, then, $(\mathcal{G}, \unlhd)$ is $\mathcal{W} \mathcal{Q O}$.

Proof. Assume an infinite sequence of named gaps $A=$ $\left(\mathrm{ph},\left(1 \mathrm{w}_{1}, \mathrm{ls}_{1}, \mathrm{uw}_{1}, \mathrm{us}_{1}\right)\right),\left(\mathrm{ph},\left(\mathrm{lw}_{2}, \mathrm{ls}_{2}, \mathrm{uw}_{2}, \mathrm{us}_{2}\right)\right), \ldots$ with the same phaser variable ph. In both cases where $A$ is free or $B$-good, from the sequence $C=\left(1 w_{1}, l s_{1}, u_{1}, u_{s_{1}}\right),\left(1 w_{2}, l s_{2}, u_{2}, u_{2}\right), \ldots$ we can extract an infinitely increasing sequence $D=D_{1}, D_{2}, \ldots$ where $\mathrm{uw}_{i}=\mathrm{uw}_{j}$ and $\mathrm{us}_{i}=\mathrm{us}_{j}$ for all $i, j \geq 1$. In the sequence ( $\left.\mathrm{ph}, D_{1}\right),\left(\mathrm{ph}, D_{2}\right), \ldots$, for every $1 \leq i<j$ we have $\left(\mathrm{ph}, D_{i}\right) \unlhd\left(\mathrm{ph}, D_{j}\right)$. This proves $(G, \unlhd)$ is $\mathcal{W} \mathcal{Q O}$.

A task state of dimension $K$ is any tuple in $\left(\mathrm{S} \times \mathcal{G}^{K}\right)$ where $K$ is a natural in $\mathbb{N}$ (corresponding to the number of phasers in the constraint to be encoded). We write $\left(\mathrm{s}, g_{1}, \ldots, g_{K}\right) \leq\left(\mathrm{s}^{\prime}, g_{1}^{\prime}, \ldots, g_{K^{\prime}}^{\prime}\right)$ for two task states to mean that 
they have the same dimension (i.e., $\left.K=K^{\prime}\right)$, that $\left(\mathrm{s} \neq \mathrm{s}^{\prime} \Rightarrow \mathrm{s}=*\right)$, and that $g_{k} \unlhd g_{k}^{\prime}$ for each $k: 1 \leq k \leq K$.

Lemma 7. $\left(\left(\mathrm{S} \times \mathcal{G}^{K}\right), \leq\right)$ is $\mathcal{W} \mathcal{Q O}$ in case $\mathcal{G}$ is good.

Proof. By Lemma 6, we have that if $\mathcal{G}$ is a good set of named gaps, then, $(G, \unlhd)$ is $\mathcal{W} \mathcal{Q O}$. Using Higman's lemma [72], finite words over a set ordered by a $\mathcal{W} \mathcal{Q O}$ are also $\mathcal{W} \mathcal{Q O}$. Moreover, $\mathrm{S}$ is a finite set and does not affect $\mathcal{W Q O}$.

Let $\mathcal{M}\left(\mathrm{S} \times \mathcal{G}^{K}\right)$ be the set of finite multisets over $\left(\mathrm{S} \times \mathcal{G}^{K}\right)$. We write $A_{\forall \leq \exists} B$, for $A$ and $B$ two multisets in $\mathcal{M}\left(\mathrm{S} \times \mathcal{G}^{K}\right)$, if each element $a \in A$ can be mapped to an element $b \in B$ for which $a \leq b$.

Lemma 8. $\left(\mathcal{M}\left(\mathrm{S} \times \mathcal{G}^{K}\right), \forall_{\exists}\right)$ is $\mathcal{W} \mathcal{Q O}$ if $\mathcal{G}$ is good.

Proof. Consider an arbitrary infinite sequence $A=A_{1}, A_{2}, \ldots$ of elements in $\mathcal{M}\left(\mathrm{S} \times \mathcal{G}^{K}\right)$. We build a sequence $B=B_{1}, B_{2}, \ldots$ where each $B_{i}$ is an arbitrary word over the elements of $A_{i}$ for $i \geq 1$. Lemma 7 together with Higman's lemma [72] ensures there exists $0 \leq i<j$ such that $B_{i}$ can be obtained from $B_{j}$ by possibly deleting some elements of $B_{j}$. Hence, $A_{i} \forall \unlhd_{\exists} A_{j}$.

We write $A_{\exists} \leq_{\forall} B$, for $A$ and $B$ finite multisets in $\mathcal{M}\left(\mathrm{S} \times \mathcal{G}^{K}\right)$, to mean that each $b \in B$ can be mapped to some $a \in A$ for which $a \leq b$. Rado's structure $[76,98]$ shows that $\left(\mathcal{M}(S), \exists_{\exists} \leq_{\forall}\right)$ need not be $\mathcal{W} \mathcal{Q O}$ just because $\leq$ is $\mathcal{W} \mathcal{Q O}$ over $S$. Still, we establish the following result:

Lemma 9. $\left(\mathcal{M}\left(\mathrm{S} \times \mathcal{G}^{K}\right), \exists \leq_{\forall}\right)$ is $\mathcal{W} \mathcal{Q O}$ if $\mathcal{G}$ is good.

Proof. We proceed by contradiction. Assume, without loss of generality, an infinite sequence $\left\langle A_{1}, A_{2}, \ldots\right\rangle$ of $\leq$-minimal multisets in $\mathcal{M}\left(\mathrm{S} \times \mathcal{G}^{K}\right)$ such that $A_{j} \exists \nless \forall \quad A_{i}$ for all $1 \leq j<i$. Notice that:

1. for each $i: 1 \leq j<i$, we can identify an element $a_{i}^{\neg^{j}} \in A_{i}$ such that $a_{j} \npreceq a_{i}^{\urcorner^{j}}$ for any $a_{j} \in A_{j}$. We sometimes write $a_{i}^{\neg^{j}}$ as $\left(\mathrm{s}_{i}^{\neg j}, a_{i}^{\neg j}[1], a_{i}^{\neg j}[2], \ldots, a_{i}^{\neg j}[K]\right)$, where $a_{i}^{\neg j}[k]$ is the $k^{t h}$ component of $a_{i}^{\neg j}$.

2. by the definition of $\left(\mathrm{S} \times \mathcal{G}^{K}, \leq\right)$ and the fact that it is a $\mathcal{W} \mathcal{Q O}$, we can extract a subsequence $\left\langle A_{i_{1}}, A_{i_{2}}, \ldots\right\rangle$ of $\left\langle A_{1}, A_{2}, \ldots\right\rangle$ associated to a sequence of partial mappings $\left(b_{j}:\{1, \ldots, K\} \rightarrow \mathbb{N}\right)$ such that for each $j: 1 \leq j$ :

a) the $j$-sequence $a_{i_{1}}^{\neg j} \leq a_{i_{2}}^{\neg j} \leq a_{i_{3}}^{\neg j} \leq \ldots$ is $\leq$-monotone,

b) each $j$-sequence has a constant control, i.e., $\mathbf{s}^{\neg j}=\mathbf{s}_{i_{1}}^{\neg j}=\mathbf{s}_{i_{2}}^{\neg j}=\ldots$, 
c) for each dimension $k: 1 \leq k \leq K$, the projection of a $j$-sequence $a_{i_{1}}^{\neg j} \leq a_{i_{2}}^{\neg j} \leq a_{i_{3}}^{\neg j} \leq \ldots$ on $k$ is either constant or strictly increasing, i.e.,:

i. $k \in \operatorname{dom}\left(b_{j}\right)$ implies $a_{i}^{\neg j}[k]=b_{j}(k)$ for all $i: j<i$

ii. $k \notin \operatorname{dom}\left(b_{j}\right)$ implies $a_{i}^{\neg j}[k] \unlhd a_{i^{\prime}}^{\neg j}[k]$ but $a_{i^{\prime}}^{\neg j}[k] \nsupseteq a_{i}^{\neg j}[k]$ for any $i, i^{\prime}: 1 \leq j<i<i^{\prime}$

We rename the sequence $\left\langle A_{i_{1}}, A_{i_{2}}, \ldots\right\rangle$ into $\left\langle A_{1}, A_{2}, \ldots\right\rangle$. We obtain a $\exists \leq_{\forall}$-bad sequence $\left\langle A_{1}, A_{2}, \ldots\right\rangle$ of $\leq$-minimal sets satisfying the constraints depicted in the following figure:

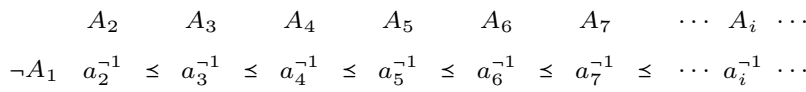

$$
\begin{aligned}
& \neg A_{2} \quad a_{3}^{-2} \leq a_{4}^{-2} \leq a_{5}^{-2} \leq a_{6}^{-2} \leq a_{7}^{2} \leq \cdots a_{i}^{-2} \cdots \\
& \neg A_{3} \quad a_{4}^{\neg 3} \leq a_{5}^{\neg 3} \leq a_{6}^{\neg^{3}} \leq a_{7}^{\neg^{3}} \leq \cdots a_{i}^{\neg^{3}} \cdots \\
& \neg A_{4} \quad a_{5}^{\neg 4} \leq a_{6}^{\neg^{4}} \leq a_{7}^{\neg^{4}} \leq \cdots a_{i}^{\neg^{4}} \cdots \\
& \neg A_{5} \quad a_{6}^{-5} \leq a_{7}^{-5} \leq \cdots a_{i}^{-5} \cdots \\
& \neg A_{6} \quad a_{7}^{\neg 6} \leq \cdots a_{i}^{\neg 6} \ldots \\
& \neg A_{j} \quad \cdots a_{i}^{\neg^{j}} \ldots
\end{aligned}
$$

Some observations about the $a_{i}^{\neg^{j}}$ elements:

1. For any $i, j: 1 \leq j<i, a_{i}^{\neg j} \in A_{i}$ and $a_{j} \nless a_{i}^{\neg j}$ for any $a_{j} \in A_{j}$.

2. For any $i, i^{\prime}, j: 1 \leq j<i<i^{\prime}, a_{i}^{\neg j} \leq a_{i^{\prime}}^{\neg j}$ with $a_{i}^{\neg j}[k]=a_{i^{\prime}}^{\neg j}[k]$ iff $k \in \operatorname{dom}\left(b_{j}\right)$

3. For any $i, j, j^{\prime}: 1 \leq j^{\prime}<j<i$ and $i^{\prime}: j \leq i^{\prime}: a_{i^{\prime}}^{\neg j^{\prime}} \npreceq a_{i}^{\neg j}$.

Observations (1) and (2) are obtained by construction. Suppose observation (3) does not hold, i.e., suppose $a_{i^{\prime}}^{\neg j^{\prime}} \leq a_{i}^{\neg j}$ for some $i, j, j^{\prime}: 1 \leq j^{\prime}<j<i$ and $i^{\prime}: j \leq i^{\prime}$. Observation (2) and reflexivity of $\leq$ ensure $a_{j}^{\neg j^{\prime}} \leq a_{i^{\prime}}^{\neg j^{\prime}}$. By transitivity of $\leq$, we get $a_{j}^{\neg j^{\prime}} \leq a_{i}^{\neg j}$. This contradicts observation (1). Hence observation (3) also holds.

Now, observe that the domain $D_{j}$ of $b_{j}$, for any $j: 1 \leq j$, is a subset of $\{1, \ldots, K\}$. Since this domain is finite, there is an infinite number of indices $j: 1 \leq j$ with the same domain $D \subseteq\{1, \ldots, K\}$. In other words, an infinite number of $j$-sequences are constants on the same dimensions $D$. We can therefore extract a sequence $\left\langle j_{1}, j_{2}, \ldots\right\rangle$ of $j$-sequences that is increasing on each dimension in $D$ (i.e., $j_{a} \leq j_{b} \Longrightarrow$ for each $\left.k \in D . b_{j_{a}}(k) \leq b_{j_{b}}(k)\right)$. In order for observation (3) to hold (i.e., $a_{i^{\prime}}^{j^{\prime}} \npreceq a_{i}^{\neg j}$ for any $i, j, j^{\prime}: 1 \leq j^{\prime}<j<i$ 
and $i^{\prime}: j \leq i^{\prime}$, we need to have some dimensions on which the $j$-sequences $\left(a_{i}^{\neg j}\right)_{j_{1}, j_{2}, \ldots}$ do not increase, these would be the dimensions in $\{1, \ldots, K\} \backslash D$. But for these dimensions, $\left(a_{i}^{\neg j}\right)_{i: 1,2, \ldots}$ is strictly increasing with $i$. This again contradicts observation (3).

Lemma 9 will be used in Lemma 11 to show that an entailment relation on encodings of $K$-dimension-bounded and $B$-good constraints corresponds to a stronger relation than 5 and is $\mathcal{W} \mathcal{Q O}$ hence, establishing Theorem 13. First, we introduce encodings of constraints and an entailment relation on them.

Encodings of constraints. Given a finite set $Q$ and an associated total order $<_{Q}$, we write $Q_{[i]} \in Q$ to mean the element of $Q$ with $<_{Q}$-index $i \epsilon$ $\{1, \ldots,|Q|\}^{2}$. For instance, given a finite set of phasers $\mathcal{P}$ and an associated total order $<_{\mathcal{P}}$, we write $\mathcal{P}_{[k]}$ to mean the phaser with $<_{\mathcal{P}}$-index $k$ in $\mathcal{P}$. The encoding of a constraint $\varphi=(\mathcal{T}, \mathcal{P}, 6 v$, pc, gap, egap $)$ with respect to total orders $<_{\mathcal{P}}$ and $<_{\mathcal{T}}$, written $\operatorname{enc}\left(\varphi,<_{\mathcal{T}},<_{\mathcal{P}}\right)$, is a tuple $(6 v, a c c, e n v)$ where:

1. 6v $: \mathrm{B} \rightarrow \mathbb{B} \cup\{*\}$ is the same as in $\varphi$,

2. acc $:\{1, \ldots,|\mathcal{T}|\} \rightarrow\left(\mathrm{S} \times \mathcal{G}^{|\mathcal{P}|}\right)$ associates each task $\mathcal{T}_{[i]}$ to a tuple $\operatorname{acc}(i)=$ $\left(\mathrm{s}, g_{1}, \ldots, g_{|\mathcal{}|}\right)$ where $p c\left(\mathcal{T}_{[i]}\right)=\mathrm{s}$ and $g a p\left(\mathcal{T}_{[i]}\right)\left(\mathcal{P}_{[j]}\right)=g_{j}$ for each phaser $\mathcal{P}_{[j]}$ with index $j$ in $\mathcal{P}$,

3. env $:\{1, \ldots,|\mathscr{P}|\} \rightarrow \mathbb{N}^{2}$ associates to each phaser $\mathcal{P}_{[j]}$ in $\mathcal{P}$ the pair env $(j)=$ $\operatorname{egap}\left(\mathcal{P}_{[j]}\right)$.

Observe that if two constraints result in the same encoding, then they can be obtained from each other by renaming the tasks and the phasers. As a consequence, if a constraint is free (respectively $B$-gap-bounded or $B$ good), then all constraints resulting in the same encoding will also be free (respectively, $B$-gap-bounded or $B$-good). We define the dimension of an encoding $(6 v, a c c, e n v)$ to be the size of the domain of $e n v$ (i.e., the dimension of an encoded constraint). A (possibly infinite) set of encodings $E$ is said to be free (respectively, $B$-gap-bounded or $B$-good) if all constraints encoded by any of its elements are free (respectively, $B$-gap-bounded or $B$-good). The set is said to be $K$-dimension-bounded if there is natural $K$ in $\mathbb{N}$ that is larger than the dimension of any of its elements.

Entailment of Encodings. Assume two encodings ( $6 v, a c c, e n v)$ and $\left(b v^{\prime}, a c c^{\prime}, e n v^{\prime}\right)$ with acc $:\{1, \ldots, L\} \rightarrow\left(\mathrm{S} \times \mathcal{G}^{M}\right)$, env $:\{1, \ldots, M\} \rightarrow \mathbb{N}^{2}$, $a c c^{\prime}:\left\{1, \ldots, L^{\prime}\right\} \rightarrow\left(\mathrm{S} \times \mathcal{G}^{M^{\prime}}\right)$, env $:\left\{1, \ldots, M^{\prime}\right\} \rightarrow \mathbb{N}^{2}$. Write $(6 v, a c c, e n v) \leq_{e}$ $\left(6 v^{\prime}, a c c^{\prime}, e n v^{\prime}\right)$ iff:

1. for each $\mathrm{v}^{b} \in \mathrm{B},\left(6 v\left(\mathrm{v}^{b}\right) \neq 6 v^{\prime}\left(\mathrm{v}^{b}\right)\right) \Longrightarrow\left(6 v\left(\mathrm{v}^{b}\right)=*\right)$, and

\footnotetext{
${ }^{2}$ the $<_{Q}$-index of an element $q$ in $Q$ is $1+\mid\left\{q^{\prime} \mid q^{\prime} \in Q\right.$ and $\left.q^{\prime}<_{Q} q\right\} \mid$
} 
2. $M^{\prime}=M$ and there is a surjection $h:\left\{1, \ldots, L^{\prime}\right\} \rightarrow\{1, \ldots, L\}$ such that:

a) $\operatorname{acc}(h(i)) \unlhd a c c^{\prime}(i)$ for each index $i \in\left\{1, \ldots, L^{\prime}\right\}$,

b) $e n v(j) \leq_{2} e n v^{\prime}(j)^{3}$ for each index $j \in\left\{1, \ldots, M^{\prime}\right\}$,

Lemma 10. Let $\varphi=(\mathcal{T}, \mathcal{P}, 6 v$, pc,gap, egap $)$ and $\varphi^{\prime}=$ $\left(\mathcal{T}^{\prime}, \mathcal{P}^{\prime}, b v^{\prime}, p c^{\prime}, g a p^{\prime}, e g a p^{\prime}\right) . \quad$ If $\operatorname{enc}\left(\varphi,<_{\mathcal{T}},<_{\mathcal{P}}\right)=(6 v, a c c, e n v)$ and $\operatorname{enc}\left(\varphi^{\prime},<_{\mathcal{T}^{\prime}},<_{\mathcal{P}^{\prime}}\right)=\left(6 v^{\prime}, a c c^{\prime}, e n v^{\prime}\right)$, then $(6 v, a c c, e n v) \leq_{e} \quad\left(6 v^{\prime}, a c c^{\prime}, e n v^{\prime}\right) i m$ plies $\varphi \sqsubseteq \varphi^{\prime}$.

Proof. From $(6 v, a c c, e n v) \leq_{e}\left(6 v^{\prime}, a c c^{\prime}, e n v^{\prime}\right)$ we deduce $|\mathcal{P}|=\left|\mathcal{P}^{\prime}\right|$ and the existence of a surjection $h:\left\{1, \ldots,\left|\mathcal{T}^{\prime}\right|\right\} \rightarrow\{1, \ldots,|\mathcal{T}|\}$, such that $\operatorname{acc}(h(i)) \unlhd \operatorname{acc}^{\prime}(i)$ for each $i \in\left\{1, \ldots,\left|\mathcal{T}^{\prime}\right|\right\}$, and env $(j) \leq_{2} e n v^{\prime}(j)$ for each $j \in\left\{1, \ldots,\left|\mathcal{P}^{\prime}\right|\right\}$.

1. $6 v=6 v^{\prime}$, hence, for each $\mathrm{v}^{b} \in \mathrm{B},\left(6 v\left(\mathrm{v}^{b}\right) \neq 6 v^{\prime}\left(\mathrm{v}^{b}\right) \Longrightarrow 6 v\left(\mathrm{v}^{b}\right)=*\right)$.

2. let $\tau: \mathcal{T}^{\prime} \rightarrow \mathcal{T}$ with $\tau\left(\mathcal{T}^{\prime}{ }_{[i]}\right)=\mathcal{T}_{[h(i)]}$ for each $\mathcal{T}^{\prime}{ }_{[i]}$ in $\mathcal{T}^{\prime}$. Let $\pi: \mathcal{P}^{\prime} \rightarrow \mathcal{P}$ with $\pi\left(\mathcal{P}_{[j]}^{\prime}\right)=\mathcal{P}_{[j]}$ for each $\mathcal{P}_{[j]}^{\prime}$ in $\mathcal{P}^{\prime}$. Observe $\tau$ is surjective and $\pi$ is bijective.

3. for each $i \in\left\{1, \ldots,\left|\mathcal{T}^{\prime}\right|\right\}$, we have that $\operatorname{acc}(h(i)) \unlhd a c c^{\prime}(i)$. By definition, $\operatorname{acc}(h(i))$ is the tuple $\left(p c\left(\mathcal{T}_{[h(i)]}\right), \operatorname{gap}\left(\mathcal{T}_{[h(i)]}\right)\left(\mathcal{P}_{[1]}\right), \ldots, \operatorname{gap}\left(\mathcal{T}_{[h(i)]}\right)\left(\mathcal{P}_{[|\mathcal{P}|]}\right)\right)$ and $\operatorname{acc}^{\prime}(i)$ is the tuple $\left(p c^{\prime}\left(\mathcal{T}^{\prime}{ }_{[i]}\right), \operatorname{gap}^{\prime}\left(\mathcal{T}^{\prime}{ }_{[i]}\right)\left(\mathcal{P}_{[1]}^{\prime}\right), \ldots, \operatorname{gap}^{\prime}\left(\mathcal{T}^{\prime}{ }_{[i]}\right)\left(\mathcal{P}_{\left[\left|\mathcal{P}^{\prime}\right|\right]}\right)\right)$ where $\mathcal{P}=\left\{\mathcal{P}_{[1]}, \ldots, \mathcal{P}_{[|\mathcal{P}|]}\right\}$ and $\mathcal{P}^{\prime}=\left\{\mathcal{P}_{[1]}^{\prime}, \ldots, \mathcal{P}_{\left[\left|\mathcal{P}^{\prime}\right|\right]}^{\prime}\right\}$. By definition of $\unlhd$, we get:

a) $p c\left(\mathcal{T}_{[h(i)]}\right) \neq p c^{\prime}\left(\mathcal{T}^{\prime}{ }_{[i]}\right) \Longrightarrow p c\left(\mathcal{T}_{[h(i)]}\right)=*$. Since $\tau\left(\mathcal{T}^{\prime}{ }_{[i]}\right)=\mathcal{T}_{[h(i)]}$, we get $p c\left(\tau\left(\mathcal{T}^{\prime}{ }_{[i]}\right)\right) \neq p c^{\prime}\left(\mathcal{T}^{\prime}{ }_{[i]}\right) \Longrightarrow p c\left(\tau\left(\mathcal{T}^{\prime}{ }_{[i]}\right)\right)=*$ for $\mathcal{T}^{\prime}{ }_{[i]}$ in $\mathcal{T}^{\prime}$.

b) $\operatorname{gap}\left(\mathcal{T}_{[h(i)]}\right)\left(\mathcal{P}_{[j]}\right) \unlhd \operatorname{gap}^{\prime}\left(\mathcal{T}^{\prime}{ }_{[i]}\right)\left(\mathcal{P}_{[j]}^{\prime}\right)$ for each $j \in\left\{1, \ldots,\left|\mathcal{P}^{\prime}\right|\right\}$. Since $\mathcal{P}_{[j]}^{\prime}=\mathcal{P}_{[j]}$, and if $\operatorname{gap}\left(\mathcal{T}_{[h(i)]}\right)\left(\mathcal{P}_{[j]}\right)=$ (ph, val) and $\operatorname{gap}^{\prime}\left(\mathcal{T}_{[i]}^{\prime}\right)\left(\mathcal{P}_{[j]}^{\prime}\right)=\left(\mathrm{ph}^{\prime}, \operatorname{val}^{\prime}\right)$, we deduce that:

i. $\left(\mathrm{ph} \neq \mathrm{ph}^{\prime}\right) \Longrightarrow(\mathrm{ph}=*)$

ii. $($ val $=\mathrm{nreg}) \Longleftrightarrow\left(\mathrm{val}^{\prime}=\mathrm{nreg}\right)$

iii. if val $=(1 \mathrm{w}, 1 \mathrm{~s}, \mathrm{uw}, \mathrm{us})$ and $\mathrm{val}^{\prime}=\left(1 \mathrm{w}^{\prime}, 1 \mathrm{~s}^{\prime}, \mathrm{uw}^{\prime}, \mathrm{us}^{\prime}\right)$, then $1 \mathrm{w} \leq$ $1 w^{\prime}$ and $l s \leq l s^{\prime}$ and $u w^{\prime} \leq u w$ and $u s^{\prime} \leq u s$

4. for each $j \in\left\{1, \ldots,\left|\mathcal{P}^{\prime}\right|\right\}$, we have $e n v(j) \leq_{2} e n v^{\prime}(j)$. By definition, $\operatorname{env}(j)=\operatorname{egap}\left(\mathcal{P}_{[j]}\right)$ and $\operatorname{env}^{\prime}(j)=\operatorname{egap}^{\prime}\left(\mathcal{P}_{[j]}^{\prime}\right)$. Since $\pi\left(\mathcal{P}_{[j]}^{\prime}\right)=\mathcal{P}_{[j]}$, we deduce that $\operatorname{egap}\left(\mathcal{P}_{[j]}\right) \leq_{2} \operatorname{egap}\left(\mathcal{P}_{[j]}^{\prime}\right)$ for each $j \in\left\{1, \ldots,\left|\mathcal{P}^{\prime}\right|\right\}$.

\footnotetext{
${ }^{3} \leq_{k}$ is the component-wise ordering on tuples of size $k$.
} 
Lemma 11. $\left(E, \leq_{e}\right)$ is $\mathcal{W} \mathcal{Q O}$ if the set $E$ of encodings is K-dimensionbounded and $B$-good for some pre-defined $K, B \in \mathbb{N}$.

Proof. Assume a $K$-dimension-bounded set $E$ of $B$-good encodings and an infinite sequence $S_{1}=\left\langle\left(b v_{1}, a c c_{1}, e n v_{1}\right),\left(b v_{2}, a c c_{2}, e n v_{2}\right), \ldots\right\rangle$. We show the existence of $i, j: 1 \leq i<j$ for which $\left(b v_{i}, a c c_{i}, e n v_{i}\right) \leq_{e}\left(b v_{j}, a c c_{j}, e n v_{j}\right)$. Dimensionboundedness of $E$ ensures there are infinitely many encodings in $S_{1}$ with the same dimension, say $K$. We extract the subsequence $S_{2}$ consisting of all encodings with dimension $K$ in $S_{1}$. In addition, observe that the set of possible valuations of the Boolean variables is finite. We can, therefore, extract from $S_{2}$ an infinite subsequence $S_{3}$ where all elements share the same valuation of the Boolean variables. Let us rewrite $S_{3}$, for simplicity, as the sequence $\left\langle\left(6 v_{1}, a c c_{1}, e n v_{1}\right),\left(b v_{2}, a c c_{2}, e n v_{2}\right), \ldots\right\rangle$. For each $i: 1 \leq i$, we can represent the mapping env $v_{i}$ as the tuple $\left(e n v_{i}(1), e n v_{i}(2), \ldots, e^{n} v_{i}(K)\right)$ in $\left(\mathbb{N}^{2}\right)^{K}$. Using Higman's lemma, we can extract from $S_{3}$ a subsequence $S_{4}$, also renamed to $\left\langle\left(b v_{1}, a c c_{1}, e n v_{1}\right),\left(b v_{2}, a c c_{2}, e n v_{2}\right), \ldots\right\rangle$ for simplicity, where $e n v_{i}(k) \leq_{2} e n v_{j}(k)$ for any $i, j: 1 \leq i<j$ and $k: 1 \leq k \leq K$.

For each mapping $a_{c c}:\left\{1, \ldots, L_{i}\right\} \rightarrow\left(\mathrm{S} \times \mathcal{G}^{K}\right)$ in $S_{4}$, we write $m_{a c c_{i}}$ to mean the multiset over $\left(\mathrm{S} \times \mathcal{G}^{K}\right)$ where the number of occurrences of an element $\left(\mathrm{s}, g_{1}, \ldots, g_{K}\right)$ coincides with the number of indices $j$ in $\left\{1, \ldots, L_{i}\right\}$ for which $\operatorname{acc}_{i}(j)=\left(\mathbf{s}, g_{1}, \ldots, g_{K}\right)$. Consider the sequence $\left\langle m_{a c c_{1}}, m_{a c c_{2}}, m_{a c c_{3}}, \ldots\right\rangle$ of elements in $\mathcal{M}\left(\mathrm{S} \times \mathcal{G}^{K}\right)$. Using the fact that $E$ is $B$-good together with Lemma 8 and 9, we deduce the existence of $i, j: i<j$ for which $m_{a c c_{i} \forall} \leq_{\exists} m_{a c c_{j}}$ and $m_{a c c_{i}} \exists \leq_{\forall} m_{a c c_{j}}$. We can therefore build a surjection $h:\left\{1, \ldots, L_{j}\right\} \rightarrow$ $\left\{1, \ldots, L_{i}\right\}$ such that $\operatorname{acc}_{i}(h(l)) \unlhd \operatorname{acc}_{j}(l)$ for each $l$ in $\left\{1, \ldots, L_{j}\right\}$.

\subsection{A Symbolic Verification Procedure}

We use the constraints from Section 5.4 as a symbolic representation in the adaptation of the classical working-list reachability analysis described below. This procedure corresponds to an instantiation of the procedure "explore" in Section 3.3. The procedure takes as arguments a set $\Phi_{b a d}$ of constraints denoting the set of bad configurations. Such sets can easily be built from the partial configurations described in Figure 5.3.

The predecessor computation rules in Figures 5.8 and 5.9 that are used by the procedure need to first concretize the given constraint to explicitly contain the required task(s), phaser(s), phaser variable(s), and control sequence(s). For this purpose, they make use of the rules concTasksPhasers (a wrapper for concretizeTask and concretizePhaser), concretizeSeq, and concretizeVar. Each concretization rule returns a (possibly empty) set of concrete constraints. Intuitively, $\operatorname{concretizeTask}(\varphi, t, \mathcal{U}, \mathcal{F})$ makes sure either $t$ that is going to be used in the computation is already in $\varphi$, or adds it as a fresh task. concretizePhaser $(\varphi, p, \mathcal{U}, \mathcal{F})$ concretizes the phaser $p$. concretizeSeq $(\varphi, t, \mathbf{s})$ makes sure the sequence of the task $t$ is $\mathbf{s}$, which is a 
requirement for the predecessor computation rule. Otherwise, it will return an empty set. Finally, concretizeVar $(\varphi, t, p, \mathrm{ph})$ ensures $t$ uses the variable ph to reference $p$, which is again required by the predecessor computation rule. In the following sections we introduce and then prove some of the characteristics of the concretization functions, predecessor computation functions, and the verification procedure.

\subsubsection{Concretization}

In this section, we introduce the concretization functions and prove some properties about them. Assume a constraint $\varphi=\left(\mathcal{T}_{\varphi}, \mathcal{P}_{\varphi}, b v_{\varphi}, p c_{\varphi}, \operatorname{gap}_{\varphi}, \operatorname{egap}_{\varphi}\right)$. We say a set $\mathcal{U}$ is in $\varphi$ if $\mathcal{U} \subseteq \mathcal{T}_{\varphi}$ and a set $\mathcal{F}$ is fresh for $\varphi$ if $\mathcal{F} \cap \mathcal{T}_{\varphi}=\varnothing$. We say concretizePhaser $\left(\varphi, p_{\varphi}, \mathcal{U}, \mathcal{F}\right)$ (Figure 5.6) concretizes the phaser $p_{\varphi}$ in $\varphi$. Rule concretize phaser 1 is used when phaser $p_{\varphi}$ is already concrete and the input constraint will be returned without any modification. Rule concretize phaser 2 adds a new phaser $p_{\varphi}$ to the constraint $\varphi$ and nondeterministically registers a set of tasks $\mathcal{T}_{1}^{\prime}$ in the concrete constraint that map to $\mathcal{T}_{1} \subseteq \mathcal{T}$ with $p_{\varphi}$. Moreover, it non-deterministically chooses a set of tasks $\mathcal{T}_{2}^{\prime}$ that map to $\mathcal{T}_{2} \subseteq \mathcal{T}$ to not be registered with $p_{\varphi}$. The task mappings $\kappa_{1}$ and $\kappa_{2}$ are used to map the tasks from the concrete constraint to constraint $\varphi$.

$\operatorname{concretizeTask}\left(\varphi, t_{\varphi}, \mathcal{U}, \mathcal{F}\right)$ (Figure 5.6) concretizes the task $t_{\varphi}$ in $\varphi$. Rule concretize task 1 is used when task $t_{\varphi}$ is already concrete and the input constraint will be returned without any modification. Rules concretize task 2 and 3 consider the case when a new task $t_{\varphi}$ will be added that copies a task $u \in \mathcal{T}$. The difference between concretize task 2 and 3 is in the fact that concretize task 3 , unlike concretize task 2 concretizes $u$ (after being renamed to $t^{\prime}$ ) as well as $t$. Rule concretize task 4 concretizes a task that is non-deterministically registered with a subset of the phasers in $\mathcal{P}_{2} \subseteq \mathcal{P}_{\varphi}$ and not registered to others. Such task needs to have phase bounds that respect the environment of the phasers in $\mathcal{P}_{2}$.

concTasksPhasers $\left(\varphi, A_{\varphi}, B_{\varphi}, \mathcal{U}, \mathcal{F}\right)$ (Figure 5.7) concretizes the phasers $A_{\varphi}$ and the tasks $B_{\varphi}$ in $\varphi$ using concretizePhaser $\left(\varphi, p_{\varphi}, \mathcal{U}, \mathcal{F}\right)$ and $\operatorname{concretizeTask}\left(\varphi, t_{\varphi}, \mathcal{U}, \mathcal{F}\right)$.

Rule concretize seq concretizes the control sequence of a task and rule concretize var concretizes the phaser name a task uses for referencing a phaser. Some facts about the the concretizations will follow.

Lemma 12. For a given constraint $\varphi=\left(\mathcal{T}_{\varphi}, \mathcal{P}_{\varphi}, b v_{\varphi}, p c_{\varphi}, \operatorname{gap}_{\varphi}\right.$, egap $\left._{\varphi}\right)$, a set $\mathcal{U}$ that is in $\varphi$, a set $\mathcal{F}$ that is fresh for $\varphi$, and a phaser $p$ that is possibly in $\mathcal{P}_{\varphi}$, concretizePhaser $(\varphi, p, \mathcal{U}, \mathcal{F})$ always terminates. Each tuple $\left(\varphi^{\prime}, \mathcal{U}^{\prime}, \mathcal{F}^{\prime}\right)$ in concretizePhaser $(\varphi, p, \mathcal{U}, \mathcal{F})$ satisfies the following:

- $\varphi \sqsubseteq \varphi^{\prime}$, 


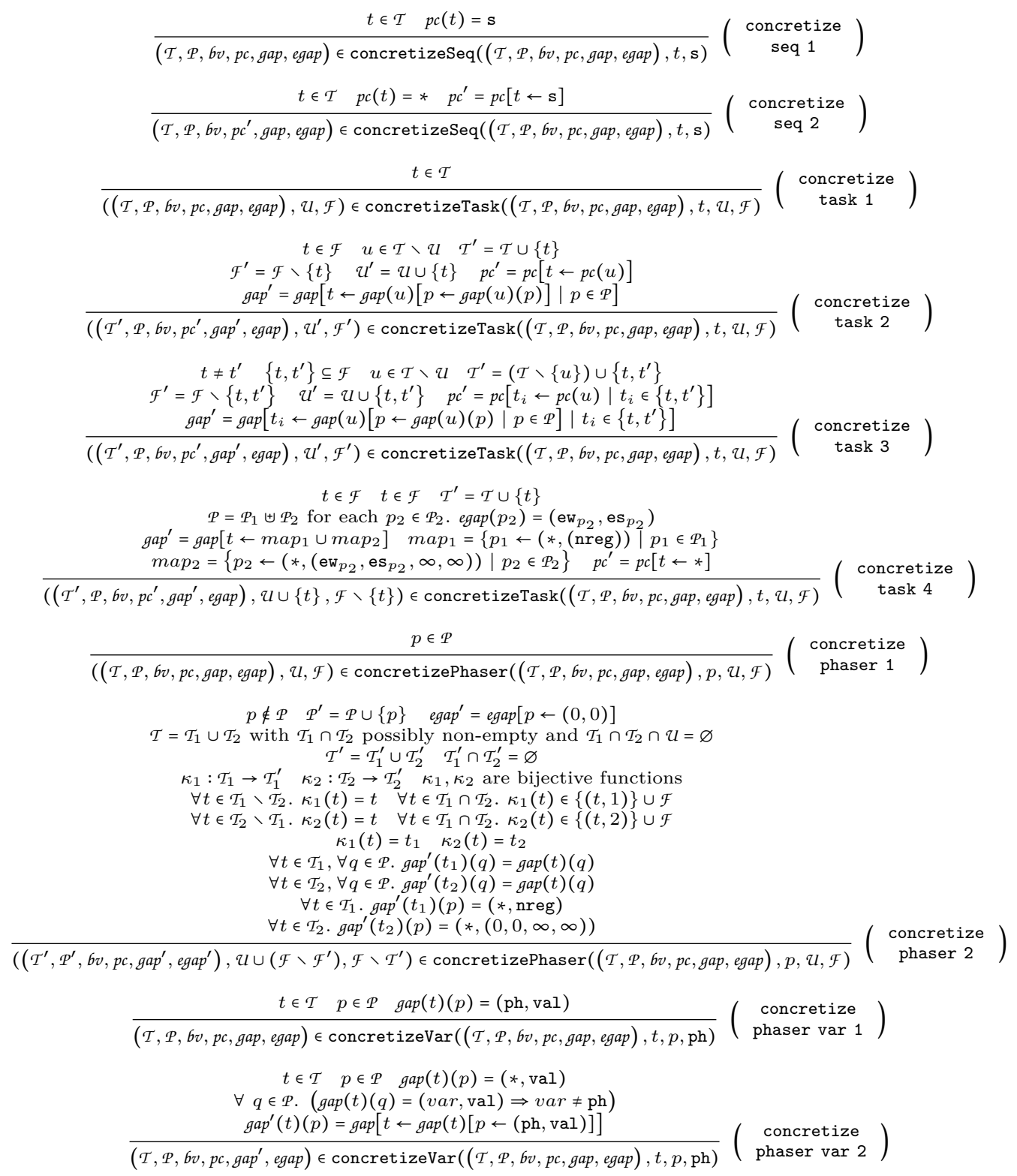

Figure 5.6: Auxiliary functions used in the predecessor computation of the parameterized Phaser instructions (Part I). The function concTasksPhasers $(\varphi, A, B, \mathcal{U}, \mathcal{F})$ concretizes the set of phasers $A$ and the set of tasks $B$ in the constraint $\varphi$. Those phasers or tasks in $A$ or $B$ that are already in $\varphi$ will be preserved. $\mathcal{U}$ is a subset of $B$, which is already concretized to unique tasks. $\mathcal{F}$ is a set of fresh task identifiers that does not intersect $B$. 


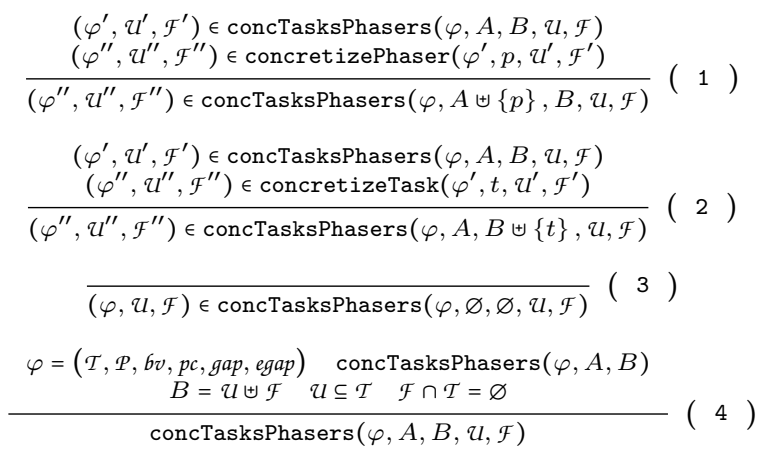

Figure 5.7: Auxiliary functions used in the predecessor computation of the parameterized Phaser instructions (Part II). The function concTasksPhasers $(\varphi, A, B, \mathcal{U}, \mathcal{F})$ concretizes the set of phasers $A$ and the set of tasks $B$ in the constraint $\varphi$. Those phasers or tasks in $A$ or $B$ that are already in $\varphi$ will be preserved.

- $\mathcal{F}^{\prime} \subseteq \mathcal{F}$ and $\mathcal{F}^{\prime} \cap \mathcal{T}_{\varphi^{\prime}}=\varnothing$,

- $\mathcal{U}^{\prime}=\mathcal{U} \cup\left(\mathcal{F} \backslash \mathcal{F}^{\prime}\right)$ and $\mathcal{U}^{\prime} \subseteq \mathcal{T}_{\varphi^{\prime}}$,

Proof. By definiton of concretize phaser 1 and 2 in Figure 5.6.

Lemma 13. For a given constraint $\varphi=\left(\mathcal{T}_{\varphi}, \mathcal{P}_{\varphi}, b v_{\varphi}, p c_{\varphi}, \operatorname{gap}_{\varphi}\right.$, egap $\left._{\varphi}\right), a$ set $\mathcal{U}$ that is in $\varphi$, a set $\mathcal{F}$ that is fresh for $\varphi$, and a task $t \in \mathcal{U} \cup \mathcal{F}$, concretizeTask $(\varphi, t, \mathcal{U}, \mathcal{F})$ always terminates. Each tuple $\left(\varphi^{\prime}, \mathcal{U}^{\prime}, \mathcal{F}^{\prime}\right)$ in concretizeTask $(\varphi, p, \mathcal{U}, \mathcal{F})$ satisfies the following:

- $\varphi \sqsubseteq \varphi^{\prime}$,

- $\mathcal{F}^{\prime} \subseteq \mathcal{F}$ and $\mathcal{F}^{\prime} \cap \mathcal{T}_{\varphi^{\prime}}=\varnothing$,

- $\mathcal{U}^{\prime}=\mathcal{U} \cup\left(\mathcal{F} \backslash \mathcal{F}^{\prime}\right)$ and $\mathcal{U}^{\prime} \subseteq \mathcal{T}_{\varphi^{\prime}}$,

Proof. By definition of concretize task 1, 2, 3, and 4 in Figure 5.6.

Observe that concretizeVar $(\varphi, t, p, \mathrm{ph})$ and concretizeSeq $(\varphi, t, \mathbf{s})$ in Figure 5.6 do not change the tasks, phasers, and phases. One can show that after calling them, the identity relations on $\mathcal{T}$ and $\mathcal{P}$ witness $\varphi \sqsubseteq \varphi^{\prime}$.

Lemma 14. For a given constraint $\varphi=\left(\mathcal{T}_{\varphi}, \mathcal{P}_{\varphi}, 6 v_{\varphi}, p c_{\varphi}, \operatorname{gap}_{\varphi}\right.$, egap $\left.\varphi\right)$, a set $\mathcal{U}$ that is in $\varphi$, a set $\mathcal{F}$ that is fresh for $\varphi$, a task $t \in \mathcal{T}_{\varphi}$, a phaser variable $\mathrm{ph}$, a phaser $p \in \mathcal{P}_{\varphi}$, and $\mathrm{s}$, concretizeVar $(\varphi, t, p, \mathrm{ph})$ and $\operatorname{concretizeSeq}(\varphi, t, \mathrm{~s})$ always terminate and will respectively generate a singleton $\left\{\varphi^{\prime}\right\}$ such that the constraint $\varphi^{\prime}$ satisfies $\varphi \sqsubseteq \varphi^{\prime}$. 
Proof. By definition of concretizeVar $(\varphi, t, p, \mathrm{ph})$ and concretizeSeq $(\varphi, t, \mathrm{~s})$ in Figure 5.6.

Lemma 15. Assume $\varphi=\left(\mathcal{T}_{\varphi}, \mathcal{P}_{\varphi}, 6 v_{\varphi}, p c_{\varphi}, \operatorname{gap}_{\varphi}, \operatorname{egap}_{\varphi}\right), A_{\varphi}$ that is a set of phaser identifiers possibly intersecting $\mathcal{P}_{\varphi}$, a set $\mathcal{U}$ that is in $\varphi$, a set $\mathcal{F}$ that is fresh for $\varphi$, and $B_{\varphi} \subseteq \mathcal{U} \cup \mathcal{F}$. concTasksPhasers $\left(\varphi, A_{\varphi}, B_{\varphi}, \mathcal{U}, \mathcal{F}\right)$ always terminates and returns a finite set of tuples. Each tuple $\left(\varphi^{\prime}, \mathfrak{u}^{\prime}, \mathcal{F}^{\prime}\right)$ in concTasksPhasers $\left(\varphi, A_{\varphi}, B_{\varphi}, \mathcal{U}, \mathcal{F}\right)$ satisfies

- $\varphi \sqsubseteq \varphi^{\prime}$,

- $\mathcal{F}^{\prime} \subseteq \mathcal{F} \backslash B_{\varphi}$ and $\mathcal{F}^{\prime} \cap \mathcal{T}_{\varphi^{\prime}}=\varnothing$,

- $\mathcal{U}^{\prime}=\mathcal{U} \cup\left(\mathcal{F} \backslash \mathcal{F}^{\prime}\right)$ and $\mathcal{U}^{\prime} \subseteq \mathcal{T}_{\varphi^{\prime}}$,

Proof. By Lemmas 12 and 13 and induction on $\left|A_{\varphi}\right|+\left|B_{\varphi}\right|$.

Definition 8 ( $\kappa$-uniquely-mapped). For a given configuration $\mathrm{c}=$

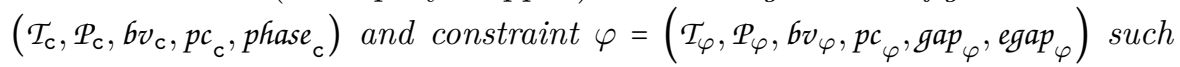
that $\kappa$ and $\pi$ witness $\mathrm{c} \in[[\varphi]]$, a task $t \in \mathcal{T}_{\mathrm{c}}$ is said to be $\kappa$-uniquely-mapped iff $\kappa(u)=\kappa(t) \Longrightarrow u=t$.

Lemma 16. Assume a configuration $\mathrm{c}=\left(\mathcal{T}_{\mathrm{c}}, \mathcal{P}_{\mathrm{c}}, 6 v_{\mathrm{c}}, p \mathcal{c}_{\mathrm{c}}\right.$, phase $\left.e_{\mathrm{c}}\right)$, a constraint $\varphi=\left(\mathcal{T}_{\varphi}, \mathcal{P}_{\varphi}, b v_{\varphi}, p c_{\varphi}, \operatorname{gap}_{\varphi}, \operatorname{egap}_{\varphi}\right)$ such that $\kappa$ and $\pi$ witness $\mathrm{c} \in[\varphi \varphi]$, a set $\mathcal{U}$ that is in $\varphi$ such that $\kappa$ uniquely maps $\kappa^{-1}(\mathcal{U})$, and a set $\mathcal{F}$ that is fresh for $\varphi$. Let $p_{\mathrm{c}}$ be an arbitrary phaser in $\mathcal{P}_{\mathrm{c}}$ and $p_{\varphi}$ be $\pi\left(p_{\mathrm{c}}\right)$ if $p_{\mathrm{c}}$ is mapped by $\pi$ or a fresh phaser, otherwise. A tuple $\left(\varphi^{\prime}, \mathcal{U}^{\prime}, \mathcal{F}^{\prime}\right)$ exists among concretizePhaser $\left(\varphi, p_{\varphi}, \mathcal{U}, \mathcal{F}\right)$ such that:

- $\mathrm{c} \in\left[\left[\varphi^{\prime}\right]\right]$ and $\kappa^{\prime}$ and $\pi^{\prime}$ witness the denotation,

- $\pi^{\prime}=\pi\left[p_{\mathrm{c}} \leftarrow p_{\varphi}\right]$,

- $\kappa^{\prime}$ uniquely maps the tasks in $\kappa^{\prime-1}\left(\mathcal{U}^{\prime}\right)$,

Proof. The concretization of $p_{\varphi}$ will be performed in one of the following ways:

- If $p_{\mathrm{c}}$ is mapped to $p_{\varphi}$ by $\pi$, the rule (concretize phaser 1 ) will return $(\varphi, \mathcal{U}, \mathcal{F})$, which is the desired tuple. Observe that the tasks are not altered, hence, the returned tuple satisfies the conditions in the lemma.

- If $p_{\mathrm{c}}$ is not mapped by $\pi$, the rule (concretize phaser 2 ) generates a set of tuples $\left(\varphi^{\prime}, \mathcal{u}^{\prime}, \mathcal{F}^{\prime}\right)$ in which the concrete constraints account for all possible registrations of the tasks in $\mathcal{T}_{\varphi}$ to the new phaser $p_{\varphi}$. A constraint $\varphi^{\prime}=\left(\mathcal{T}_{\varphi^{\prime}}, \mathcal{P}_{\varphi^{\prime}}, 6 v_{\varphi^{\prime}}, p c_{\varphi^{\prime}}, \operatorname{gap}_{\varphi^{\prime}}, \operatorname{egap}_{\varphi^{\prime}}\right)$ generated by the rule will capture 
- the tasks in $\mathcal{T}_{1} \subseteq \mathcal{T}_{\mathrm{c}}$ that were mapped by $\kappa$ but were not registered with $p_{\mathrm{c}}$ can be mapped to $\mathcal{T}_{1}^{\prime} \subseteq \mathcal{T}_{\varphi^{\prime}}$ by the task mapping $\kappa_{1}(\kappa)$.

- the tasks in $\mathcal{T}_{2} \subseteq \mathcal{T}_{\mathrm{c}}$ that were mapped by $\kappa$ and are registered with $p_{\mathrm{c}}$ can be mapped to $\mathcal{T}_{2}^{\prime} \subseteq \mathcal{T}_{\varphi^{\prime}}$ by the task mapping $\kappa_{2}(\kappa)$.

The task mapping $\kappa^{\prime}$ that maps the tasks in $\mathcal{T}_{1}$ using $\kappa_{1}(\kappa)$ and those in $\mathcal{T}_{2}$ using $\kappa_{2}(\kappa)$ and the phaser mapping $\pi^{\prime}=\pi\left[p_{\mathrm{c}} \leftarrow p_{\varphi}\right]$ witness $\mathrm{c} \vDash \varphi^{\prime}$. $\kappa^{\prime}$ by construction uniquely maps the tasks in $\kappa^{\prime-1}\left(\mathcal{U}^{\prime}\right)$.

Lemma 17. Assume a configuration $\mathrm{c}=\left(\mathcal{T}_{\mathrm{c}}, \mathcal{P}_{\mathrm{c}}, 6 v_{\mathrm{c}}, p \mathcal{c}_{\mathrm{c}}\right.$, phase $\left.\mathrm{c}_{\mathrm{c}}\right)$, a constraint $\varphi=\left(\mathcal{T}_{\varphi}, \mathcal{P}_{\varphi}, b v_{\varphi}, p c_{\varphi}, \operatorname{gap}_{\varphi}, \operatorname{egap}_{\varphi}\right)$ such that $\kappa$ and $\pi$ witness $\mathrm{c} \in[\varphi \varphi]$, a set $\mathcal{U}$ that is in $\varphi$ such that $\kappa$ uniquely maps $\kappa^{-1}(\mathcal{U})$, a set $\mathcal{F}$ that is fresh for $\varphi$, and a set $B_{\mathrm{c}}=\left(B_{\mathrm{c}_{1}} \uplus B_{\mathrm{c}_{2}}\right) \subseteq \mathcal{T}_{\mathrm{c}}$ such that $\kappa$ uniquely maps $B_{\mathrm{c}_{1}}$ and does not uniquely map $B_{\mathrm{c}_{2}}$. Let $t_{\mathrm{c}}$ be an arbitrary task in $B_{\mathrm{c}}$ and $t_{\varphi}$ be $\kappa\left(t_{\mathrm{c}}\right)$ if $t_{\mathrm{c}}$ is in $\mathcal{U}$ or a task $n \mathcal{F}$, otherwise. concretizeTask $\left(\varphi, t_{\varphi}, \mathcal{U}, \mathcal{F}\right)$ will generate a set of tuples among which there is a tuple $\left(\varphi^{\prime}, \mathcal{U}^{\prime}, \mathcal{F}^{\prime}\right)$ such that

- $\mathrm{c} \in\left[\left[\varphi^{\prime}\right]\right]$ and $\kappa^{\prime}$ and $\pi$ witness the denotation,

- $\kappa^{\prime}$ uniquely maps the tasks in $\kappa^{\prime-1}\left(\mathcal{U}^{\prime}\right)$,

Proof. The concretization of $t_{\varphi}$ will be performed in one of the following ways:

- If $t_{\mathrm{c}}$ is in $B_{\mathrm{c}_{1}}$, it is already uniquely mapped to $t_{\varphi}$, the rule (concretize task 1) will return $(\varphi, \mathcal{U}, \mathcal{F})$, which is the desired tuple.

- If $t_{\mathrm{c}}$ is mapped by $\kappa$ but is in $B_{\mathrm{c}_{2}}$, it is mapped to $\kappa\left(t_{\mathrm{c}}\right)$ but not uniquely. The rule (concretize task 2) (respectively, concretize task 3) return in this case a tuple $\left(\varphi^{\prime}, \mathcal{U}^{\prime}, \mathcal{F}^{\prime}\right)$ for which the mappings $\kappa^{\prime}=\kappa\left[t_{\mathrm{c}} \leftarrow t_{\varphi}\right]$ (respectively, $\kappa^{\prime}=\kappa\left[t_{\mathrm{c}} \leftarrow t_{\varphi}\right]\left[u_{\mathrm{c}} \leftarrow u_{\varphi}\right]$ given that $t_{\mathrm{c}}$ and $u_{\mathrm{c}}$ are both mapped to $\left.\kappa\left(t_{\mathrm{c}}\right)\right)$ and $\pi$ witnesses $\mathrm{c} \in\left[\left[\varphi^{\prime}\right]\right]$. The other claims hold by construction.

- If $t_{\mathrm{c}}$ is not mapped by $\kappa$, it is in $B_{\mathrm{c}_{2}}$. Let $\mathcal{P}_{\mathrm{c}}^{m} \subseteq \mathcal{P}_{\mathrm{c}}$ be the set of mapped phasers by $\pi$ and $\mathcal{P}_{\mathrm{c}}^{m, r} \subseteq \mathcal{P}_{\mathrm{c}}^{m}$ be the set of mapped phasers that $t_{\mathrm{c}}$ is registered with. In this case, the rule (concretize task 4) returns a set of tuples among which there is a tuple $\left(\varphi^{\prime}, \mathcal{U}^{\prime}, \mathcal{F}^{\prime}\right)$ in which $t_{\varphi}$ is a fresh task that is registered with $\pi\left(\mathcal{P}_{\mathrm{c}}^{m, r}\right)$ and not with $\pi\left(\mathcal{P}_{\mathrm{c}}^{m} \backslash \mathcal{P}_{\mathrm{c}}^{m, r}\right)$. Hence, the task and phaser mappings $\kappa^{\prime}=\kappa\left[t_{\mathrm{c}} \leftarrow t_{\varphi}\right]$ and $\pi$ witnesses $c \vDash \varphi^{\prime}$. The other claims hold by construction. 
Definition 9 (Match). Assume a tuple (c, $\left.A_{\mathrm{c}}, B_{\mathrm{c}}\right)$ with $\mathrm{c}=$ $\left(\mathcal{T}_{\mathrm{c}}, \mathcal{P}_{\mathrm{c}}, 6 v_{\mathrm{c}}, p \mathcal{c}_{\mathrm{c}}\right.$, phase $\left.e_{\mathrm{c}}\right), A_{\mathrm{c}} \subseteq \mathcal{P}_{\mathrm{c}}$, and $B_{\mathrm{c}} \subseteq \mathcal{T}_{\mathrm{c}}$ and $\left(\varphi, A_{\varphi}, B_{\varphi}, \mathcal{U}, \mathcal{F}\right)$ with $\varphi=\left(\mathcal{T}_{\varphi}, \mathcal{P}_{\varphi}, b v_{\varphi}, p c_{\varphi}, \operatorname{gap}_{\varphi}, \operatorname{egap}_{\varphi}\right), A_{\varphi}$ that is a set of phaser identifiers possibly intersecting $\mathcal{P}_{\varphi}, B_{\varphi}=\mathcal{U} \cup \mathcal{F}$, a set $\mathcal{U}$ that is in $\varphi$, and a set $\mathcal{F}$ that is fresh for $\varphi$. We say the tuple $\left(\mathrm{c}, A_{\mathrm{c}}, B_{\mathrm{c}}\right)$ matches $\left(\varphi, A_{\varphi}, B_{\varphi}, \mathcal{U}, \mathcal{F}\right)$ with respect to $\kappa$ and $\pi$ if

- $\kappa$ and $\pi$ witness $\mathrm{c} \in[[\varphi]$,

- $\kappa^{-1}(\mathcal{U})$ is $\kappa$-uniquely-mapped,

- $\left|A_{\mathrm{c}}\right|=\left|A_{\varphi}\right|$ and $\left|B_{\mathrm{c}}\right|=\left|B_{\varphi}\right|$,

- $B_{\mathrm{c}}=B_{\mathrm{c}_{1}} \uplus B_{\mathrm{c}_{2}}$,

- $\kappa\left(B_{\mathrm{c}_{1}}\right) \subseteq \mathcal{U}$, i.e., $B_{\mathrm{c}_{1}}$ is $\kappa$-uniquely-mapped,

- $\kappa$ does not uniquely map $B_{\mathrm{c}_{2}}$,

- $\left|B_{\mathrm{c}_{2}}\right|=|\mathcal{F}|$.

Lemma 18. Given $\mathrm{c}=\left(\mathcal{T}_{c}, \mathcal{P}_{\mathrm{c}}, 6 v_{\mathrm{c}}, p c_{\mathrm{c}}\right.$, phase $\left._{\mathrm{c}}\right), \varphi\left(\mathcal{T}_{\varphi}, \mathcal{P}_{\varphi}, b v_{\varphi}, p c_{\varphi}\right.$, gap $\left._{\varphi}, \operatorname{egap}_{\varphi}\right)$, and $\kappa$ and $\pi$ that witness $\mathrm{c} \in\left[[\varphi]\right.$, for any $A_{\mathrm{c}} \subseteq \mathcal{P}_{\mathrm{c}}$ and $B_{\mathrm{c}} \subseteq \mathcal{T}_{\mathrm{c}},\left(\mathrm{c}, A_{\mathrm{c}}, B_{\mathrm{c}}\right)$ matches $\left(\varphi, A_{\varphi}, B_{\varphi}, \mathcal{U}, \mathcal{F}\right)$ with respect to $\kappa$ and $\pi$ if

- $\left|A_{\mathrm{c}}\right|=\left|A_{\varphi}\right|$ and $\left|B_{\mathrm{c}}\right|=\left|B_{\varphi}\right|$,

- $A_{\varphi}=\pi\left(A_{\left.\mathrm{c}\right|_{\pi}}\right) \uplus\left(A_{\varphi} \backslash \operatorname{dom}(\pi)\right)$,

- $B_{\varphi}=\mathcal{U} \uplus \mathcal{F}, \mathcal{U} \subseteq \mathcal{T}_{\varphi}, \mathcal{F} \cap \mathcal{T}_{\varphi}=\varnothing$,

- $\kappa^{-1}(\mathcal{U})$ is $\kappa$-uniquely-mapped.

Proof. By Definition 9 and construction of $c \in[[\varphi]$.

Example 4. Let $\mathrm{c} \in[[\varphi]]$ such that $\mathrm{c}=\left(\mathcal{T}_{\mathrm{c}}, \mathcal{P}_{\mathrm{c}}, 6 v_{\mathrm{c}}, p c_{\mathrm{c}}\right.$, phase $\left.\mathrm{c}_{\mathrm{c}}\right), p_{\mathrm{c}} \in \mathcal{P}_{\mathrm{c}}, t_{\mathrm{c}} \in$ $\mathcal{T}_{c}, \varphi=\left(\mathcal{T}_{\varphi}, \mathcal{P}_{\varphi}, 6 v_{\varphi}, p c_{\varphi}, \operatorname{gap}_{\varphi}, \operatorname{egap}_{\varphi}\right)$, and $\kappa$ and $\pi$ witness the denotation. $\left(\mathrm{c},\left\{p_{\mathrm{c}}\right\},\left\{t_{\mathrm{c}}\right\}\right)$ will match some $\left(\varphi,\left\{p_{\varphi}\right\},\left\{t_{\varphi}\right\}, \mathcal{U}, \mathcal{F}\right)$ for $\mathcal{U} \cup \mathcal{F}=\left\{t_{\varphi}\right\}$.

Lemma 19. Assume a configuration $\mathrm{c}=\left(\mathcal{T}_{\mathrm{c}}, \mathcal{P}_{\mathrm{c}}, 6 v_{\mathrm{c}}, p \mathrm{c}_{\mathrm{c}}\right.$, phase $\left.{ }_{\mathrm{c}}\right)$, a constraint $\varphi=\left(\mathcal{T}_{\varphi}, \mathcal{P}_{\varphi}, b v_{\varphi}, p c_{\varphi}, \operatorname{gap}_{\varphi}, \operatorname{egap}_{\varphi}\right)$, the mappings $\kappa$ and $\pi$, and the sets $A_{\mathrm{c}}, B_{\mathrm{c}}$, $A_{\varphi}$, and $B_{\varphi}$ such that $\left(\mathrm{c}, A_{\mathrm{c}}, B_{\mathrm{c}}\right)$ matches $\left(\varphi, A_{\varphi}, B_{\varphi}, \mathcal{U}, \mathcal{F}\right)$ with respect to $\kappa$ and $\pi$. For all the tuples $\left(\varphi^{\prime}, \mathcal{U}^{\prime}, \mathcal{F}^{\prime}\right)$ in concTasksPhasers $\left(\varphi, A_{\varphi}, B_{\varphi}, \mathcal{U}, \mathcal{F}\right)$ we have that $\mathcal{U}^{\prime}=B_{\varphi}$ and $\mathcal{F}^{\prime}=\varnothing$ and there exists one such tuple that:

- $\pi^{\prime}: \operatorname{dom}(\pi) \cup A_{\mathrm{c}} \rightarrow \mathcal{P}_{\varphi^{\prime}}$,

- $\kappa^{\prime}: \operatorname{dom}(\kappa) \cup B_{\mathrm{c}} \rightarrow \mathcal{T}_{\varphi^{\prime}}$, 
- $\kappa^{\prime}$ uniquely maps $B_{\mathrm{c}}$,

- $\pi^{\prime}$ and $\kappa^{\prime}$ witness $\mathrm{c} \in\left[\left[\varphi^{\prime}\right]\right]$.

Proof. By Lemmas 16 and 17 and induction on $\left|A_{\varphi}\right|+\left|B_{\varphi}\right|$.

Lemma 20. Assume a configuration $\mathrm{c}=\left(\mathcal{T}_{\mathrm{c}}, \mathcal{P}_{\mathrm{c}}, b v_{\mathrm{c}}, p c_{\mathrm{c}}\right.$, phase $\left.{ }_{\mathrm{c}}\right)$ and a constraint $\varphi=\left(\mathcal{T}_{\varphi}, \mathcal{P}_{\varphi}, b v_{\varphi}, p c_{\varphi}\right.$, gap $_{\varphi}$, egap $\left._{\varphi}\right)$ such that $\kappa$ and $\pi$ witness $c \in[[\varphi]]$. Assume also that for some task $t_{\mathrm{c}}$ and phaser $p_{\mathrm{c}}, \kappa\left(t_{\mathrm{c}}\right)=t_{\varphi}, \pi\left(p_{\mathrm{c}}\right)=p_{\varphi}$, $p c_{\mathrm{c}}\left(t_{\mathrm{c}}\right)=\mathrm{s}$, and phase $\left(t_{\mathrm{c}}\right)\left(p_{\mathrm{c}}\right)=(\mathrm{ph}, \mathrm{val})$. concretizeVar $\left(\varphi, t_{\varphi}, p_{\varphi}, \mathrm{ph}\right)$ and concretizeSeq $\left(\varphi, t_{\varphi}, \mathrm{s}\right)$ will respectively generate a singleton $\left\{\varphi^{\prime}\right\}$ such that the constraint $\varphi^{\prime}$ satisfies $\mathrm{c} \in\left[\left[\varphi^{\prime}\right]\right]$.

Proof. By definition of concretizeVar $(\varphi, t, p, \mathrm{ph})$ and concretizeSeq $(\varphi, t, \mathrm{~s})$ in Figure 5.6.

\subsubsection{Predecessor Computation}

In this section, we formally define the predecessor computation functions in Figures 5.8 and 5.9 and prove their soundness and relative completeness.

Theorem 14. Each predecessor computation rule $\stackrel{\text { stmt }}{\longrightarrow}$ in Figures 5.9 and 5.8 is sound with respect to the semantic rules of Figure 5.2.

Proof. Assume a configuration $\mathrm{c}$ and a constraint $\varphi$ such that $\mathrm{c} \in[[\varphi]$.

newPhaser. Assume $c^{\prime} \frac{\text { ph:=newPhaser }()}{t_{c}} c$ for some $c^{\prime}$ where $p_{c}$ is the new phaser id in c. We exhibit $\varphi^{\prime}$ as well as the task and phaser mappings that witness $c^{\prime} \in\left[\left[\varphi^{\prime}\right]\right]$ such that $\varphi \stackrel{\text { ph:=newPhaser }()}{t_{\varphi}} \varphi^{\prime}$ for some $t_{\varphi}$. The rule starts by concretizing $\varphi$ according to $\mathrm{c}$ and $A_{\mathrm{c}}=\left\{t_{\mathrm{c}}\right\}$ and $B_{\mathrm{c}}=\left\{p_{\mathrm{c}}\right\}$. Lemmas 19 and 20 ensure that after concretizations, a concrete constraint $\varphi_{\text {conc }}$ is generated such that $c \in\left[\left[\varphi_{\text {conc }}\right]\right]$ with respect to some task and phaser mappings $\kappa$ and $\pi$ so that $t_{\mathrm{c}}$ is uniquely mapped to $t_{\varphi}$ by $\kappa$, and $p_{\mathrm{c}}$ is mapped to some $p_{\varphi}$ by $\pi$. The task $t_{\mathrm{c}}$ in $c^{\prime}$ creates $p_{\mathrm{c}}$, hence, it must be the only task registered with $p_{\mathrm{c}}$ or referencing it. This, combined with the definition of $\kappa$ and $\pi$, guarantees that $t_{\varphi}$ is the only task in $\varphi$ which is registered with $p_{\varphi}$ or referencing it. The new mappings $\kappa$ and $\pi^{\prime}=\pi \backslash\left\{p_{\mathrm{c}}\right\}$ witness $\mathrm{c}^{\prime} \in\left[\left[\varphi^{\prime}\right]\right]$.

signal. Assume $c^{\prime} \underset{t_{c}}{\stackrel{\text { ph.signal }()}{\longrightarrow}} \mathrm{c}$ for some $c^{\prime}$. We exhibit $\varphi^{\prime}=$ $\left(\mathcal{T}_{\varphi}^{\prime}, \mathcal{P}_{\varphi}^{\prime}, b v_{\varphi}^{\prime}, p c_{\varphi}^{\prime}, g a p_{\varphi}^{\prime}, e g a p_{\varphi}^{\prime}\right)$ as well as the task and phaser mappings that witness $c^{\prime} \in\left[\left[\varphi^{\prime}\right]\right]$ such that $\varphi \stackrel{\text { ph.signal }()}{\underset{t_{\varphi}}{\longrightarrow}} \varphi^{\prime}$ for some $t_{\varphi}$. The rule starts by 


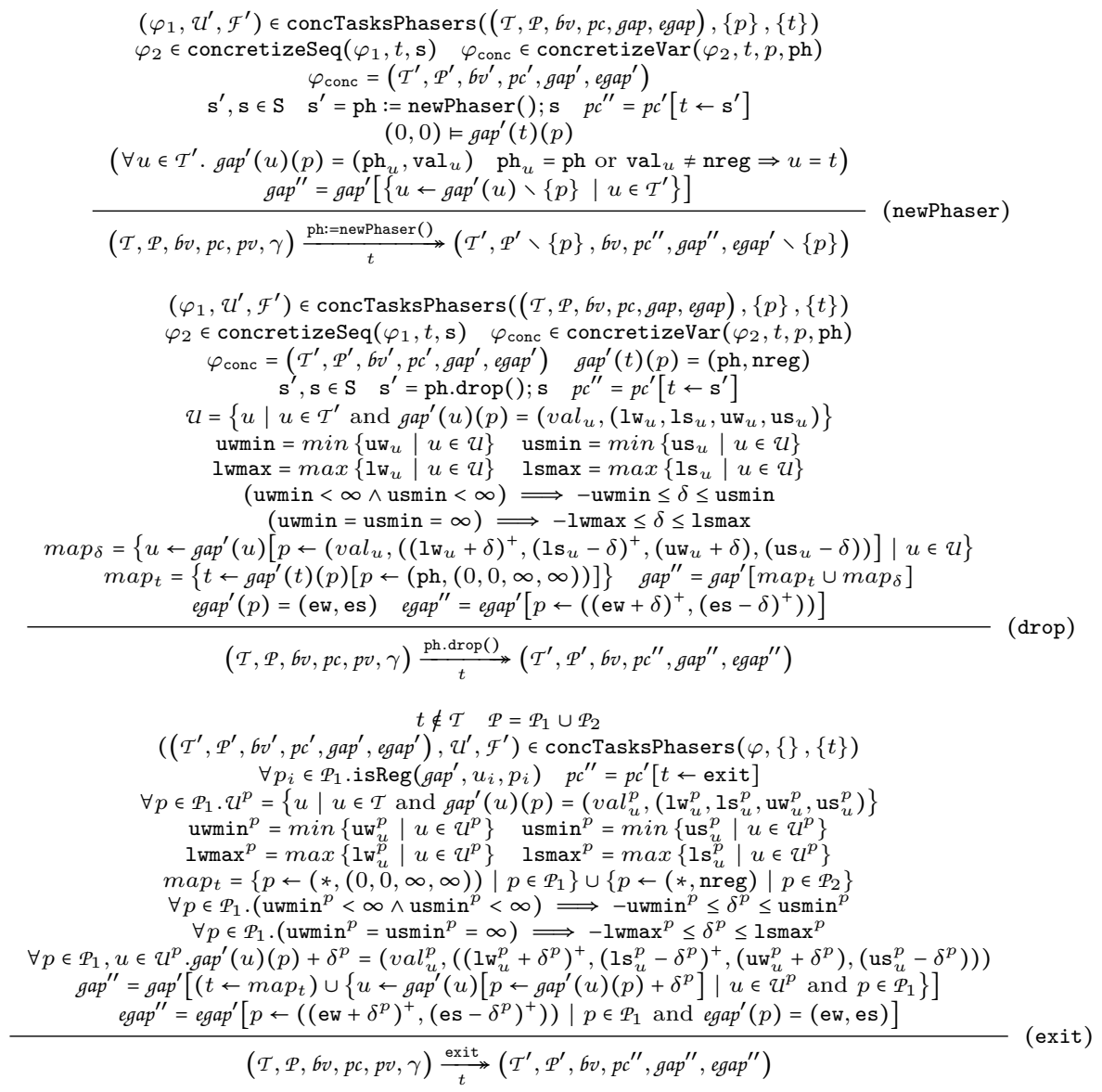

Figure 5.8: Derivation rules for computing predecessors wrt. parameterized Phaser instructions newPhaser(), ph.drop() and exit. For each rule, a task $t$ executes the statement. If $t$ does not belong to $\mathcal{T}$, it will be added by concretizing $\varphi$. Otherwise, the concretization will preserve it. In newPhaser() and ph.drop(), a phaser $p$ is required, which is either added by concretization or is preserved by it.

concretizing $t_{\varphi}$ and $p_{\varphi}$ in $\varphi$. Lemmas 19 and 20 ensure that a concrete constraint $\varphi_{\text {conc }}=\left(\mathcal{T}_{\varphi}, \mathcal{P}_{\varphi}, b v_{\varphi}, p c_{\varphi}, \operatorname{gap}_{\varphi}, \operatorname{egap}_{\varphi}\right)$ is generated such that $\mathrm{c} \in\left[\left[\varphi_{\text {conc }}\right]\right]$ with respect to some task and phaser mappings $\kappa$ and $\pi$ so that $t_{\mathrm{c}}$ is uniquely mapped to some $t_{\varphi}$ by $\kappa$, and $p_{\mathrm{c}}$ is mapped to some $p_{\varphi}$ by $\pi$. The task $t_{\mathrm{c}}$ in $c^{\prime}$ has just incremented the signal phase of the task $t_{c}$ on the phaser $p_{c}$. There is a level lev $\geq 0$ that separates the signal phases of the tasks registered with $p_{\text {concTasksPhasers }}$ from their wait phases. Incrementing the signal sig ${ }^{t_{c}}$ of 


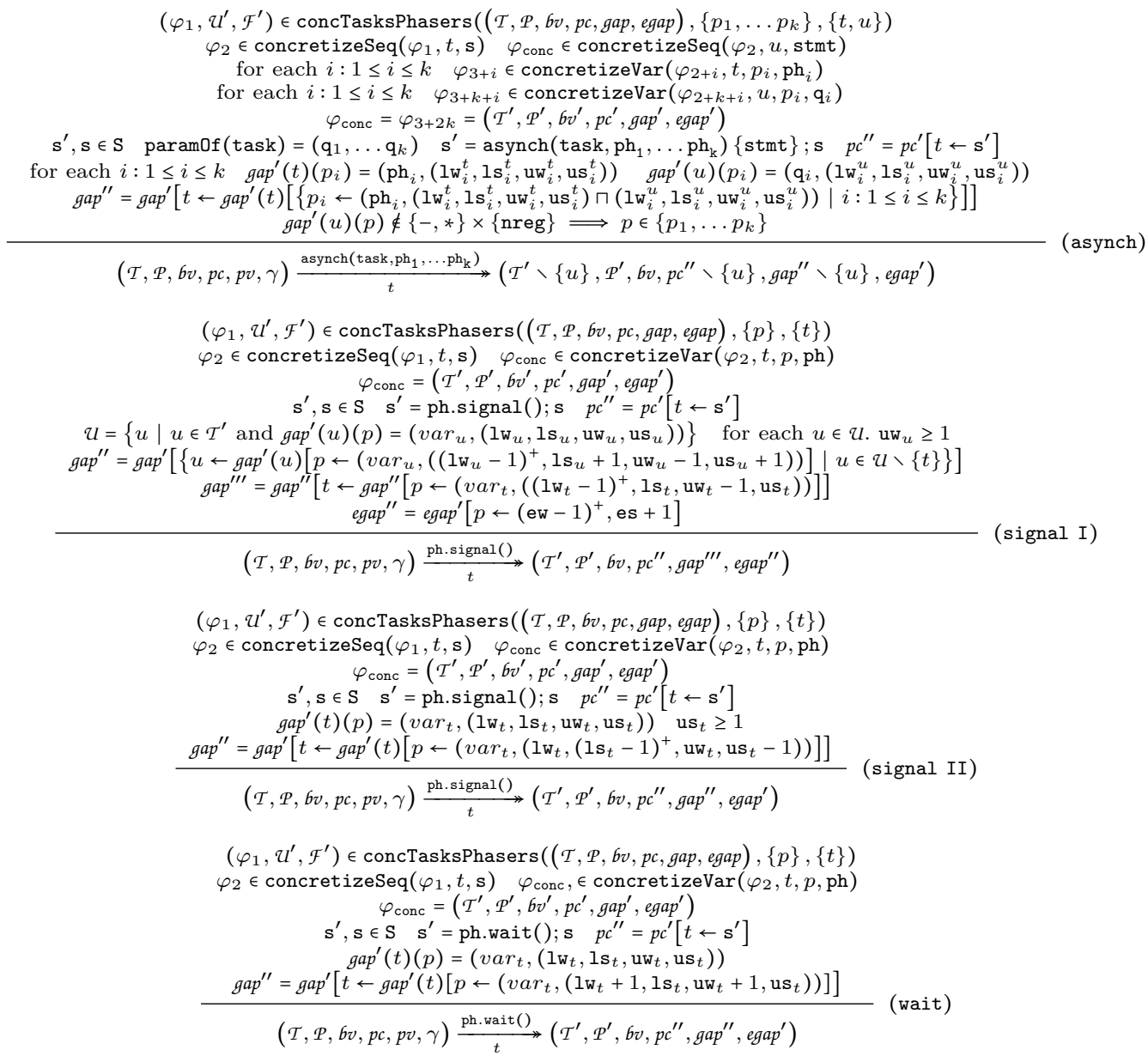

Figure 5.9: Derivation rules for predecessor computation wrt. parameterized Phaser instructions asynch(task, $\left.\mathrm{ph}_{1}, \ldots \mathrm{ph}_{\mathrm{k}}\right)\{$ stmt $\}$, ph.signal () and ph.wait(). For each rule, a task $t$ executes the statement. If $t$ does not belong to $\mathcal{T}$, it will be added by concretizing $\varphi$. Otherwise, the concretization will preserve it. In the given statements, one or more phasers are required, which are either added by concretization or are preserved by that.

task $t_{\mathrm{c}}$ on $p_{\mathrm{c}}$ satisfies the phase bounds in $\varphi_{\text {conc }}$. The case in which lev $<s i g^{t_{c}}$ can be captured by signal II (here, us $>0$ and $\left.l e \gamma^{\prime}=l e v\right)$. The case where lev $=\operatorname{sig}^{t_{\mathrm{c}}}$ can be captured with signal I (here, uw $>0$ and lev' $\left.{ }^{\prime}=l_{e v}-1\right)$. 
wait. Assume $c^{\prime} \underset{t_{c}}{\stackrel{\text { ph.wait }()}{\longrightarrow}} \mathrm{c}$ for some $\mathrm{c}^{\prime}$. We exhibit $\varphi^{\prime}$ as well as the task and phaser mappings that witness $\left.c^{\prime} \in \llbracket \varphi^{\prime}\right]$ such that $\varphi \stackrel{\text { ph.wait() }}{t_{\varphi}} \varphi^{\prime}$ for some $t_{\varphi}$. Similar to the signal rule, this rule starts by concretizing $t_{\mathrm{c}}$ and $p_{c}$ in $c$ and uniquely maps them to $t_{\varphi}$ and $p_{\varphi}$ in $\varphi_{\text {conc. The task }} t_{c}$ in $c^{\prime}$ has just incremented the wait phase of the task $t_{\mathrm{c}}$ on the phaser $p_{\mathrm{c}}$. Let $\operatorname{gap}_{\varphi}\left(t_{\varphi}\right)\left(p_{\varphi}\right)=\left(\mathrm{ph},\left(1 \mathrm{w}, \mathrm{ls}_{\mathrm{s}}\right.\right.$ uw, us $\left.)\right)$. A level lev $>0$ exists that shows the signal and wait phases of the tasks registered with $p_{\mathrm{c}}$ respect the phase bounds in $\varphi_{\text {conc }}$. The same level can be used to show that phases of $c^{\prime}$ respect the phase bounds in $\varphi^{\prime}$ that is generated by the rule.

drop. Assume $c^{\prime} \underset{t_{\mathrm{c}}}{\stackrel{\text { ph.drop() }}{\longrightarrow}} \mathrm{c}$ for some $\mathrm{c}^{\prime}$. We exhibit $\varphi^{\prime}=$ $\left(\mathcal{T}_{\varphi}^{\prime}, \mathcal{P}_{\varphi}, b v_{\varphi}, p c_{\varphi}^{\prime}, \operatorname{gap}_{\varphi}^{\prime}, \operatorname{egap}_{\varphi}\right)$ such that $\mathrm{c}^{\prime} \in\left[\left[\varphi^{\prime}\right]\right]$ and $\varphi \stackrel{\text { ph.drop() }}{\underset{t_{\varphi}}{\longrightarrow}} \varphi^{\prime}$ for some task $t_{\varphi}$. Similar to the signal rule, this rule starts by concretizing $t_{\mathrm{c}}$ and $p_{\mathrm{c}}$ in $c$ and uniquely maps them to $t_{\varphi}$ and $p_{\varphi}$ in $\varphi_{\text {conc }}$. Since $c \in\left[\left[\varphi_{\text {conc }}\right]\right]$, there exists a level $l_{e v}$ that separates signal and wait phases of the tasks registered with $p_{\varphi}$. The task $t_{c}$ in $c^{\prime}$ has just dropped the phaser $p_{c}$. By existence of $\mathrm{c}^{\prime}$, a lev' exists that takes into account the phases of $t_{\varphi}$ as well. If $\delta<-1$ wmax (respectively, $\delta>$ lsmax), we can show lev - I wmax (respectively, lev +1 smax) also separates the phases in $c^{\prime}$. Otherwise, lev $+\delta$ with -1 wmax $\leq \delta \leq 1$ smax separates the phases in $\mathbf{c}^{\prime}$. We adapt the bounds for each such $\delta$ in this case. In a similar manner, if usmin $<\infty$ and uwmin $<\infty$ for some task, then a level lev $+\delta$ with - uwmin $\leq \delta \leq$ usmin must separate the phases in $c^{\prime}$. We adapt the phase bounds to this case.

exit. Assume $c^{\prime} \underset{t_{c}}{\stackrel{\text { exit }}{\longrightarrow}}$ c for some $c^{\prime}$. Proof of soundness of the rule exit has a similar approach to that of the drop rule. The difference is that exit intuitively has to iterate through all the phasers in $\mathcal{P}_{\mathrm{c}}$ with which $t_{\mathrm{c}}$ is registered and drop them. The mappings $\kappa^{\prime}=\kappa \cup\left\{t_{\mathrm{c}} \rightarrow t_{\varphi}\right\}$ and $\pi$ witness the denotation.

asynch. Assume $c^{\prime} \stackrel{\operatorname{asynch}\left(\operatorname{task}, \mathrm{ph}_{1}, \ldots \mathrm{ph}_{\mathrm{k}}\right)\{\mathrm{s}\}}{t_{\mathrm{c}}} \mathrm{c}$ for some $c^{\prime}$ where $\operatorname{param0f}($ task $)=\left(\mathrm{q}_{1}, \ldots \mathrm{q}_{k}\right)$. The task $t_{\mathrm{c}}$ has just spawned $u_{\mathrm{c}}$ in $\mathrm{c}$. We exhibit $\varphi^{\prime}=\left(\mathcal{T}_{\varphi}^{\prime}, \mathcal{P}_{\varphi}^{\prime}, b v_{\varphi}^{\prime}, p c_{\varphi}^{\prime}, \operatorname{gap}_{\varphi}^{\prime}, \operatorname{egap}_{\varphi}^{\prime}\right)$ such that $\mathrm{c}^{\prime} \in\left[\left[\varphi^{\prime}\right]\right]$ and $\varphi \stackrel{\operatorname{asynch}\left(\operatorname{task}, \mathrm{ph}_{1}, \ldots \mathrm{ph}_{\mathrm{k}}\right)\{\mathrm{s}\}}{t_{\varphi}} \varphi^{\prime}$. The rule starts by concretizing $t_{\varphi}, u_{\varphi}$, and $p_{i_{\varphi}}$ for every $i: 1 \leq i \leq k$ in $\varphi$. Lemmas 19 and 20 ensure that a concrete constraint $\varphi_{\text {conc }}=\left(\mathcal{T}_{\varphi}, \mathcal{P}_{\varphi}, b v_{\varphi}, p c_{\varphi}, \operatorname{gap}_{\varphi}, \operatorname{egap}_{\varphi}\right)$ is generated such that $\mathrm{c} \in\left[\left[\varphi_{\text {conc }}\right]\right]$ with respect to some task and phaser mappings $\kappa$ and $\pi$ so that $t_{\mathrm{c}}$ and $u_{\mathrm{c}}$ are uniquely mapped to some $t_{\varphi}$ and $u_{\varphi}$ by $\kappa$, and $p_{i_{c}}$ is mapped to some 
$p_{i_{\varphi}}$ by $\pi$ for each $i: 1 \leq i \leq k$. The task $t_{\mathrm{c}}$ in $\mathrm{c}^{\prime}$ has just spawned the task $u_{\mathrm{c}} \cdot t_{\mathrm{c}}$ and $u_{\mathrm{c}}$ are registered with each $p_{i_{\mathrm{c}}}$ for $i: 1 \leq i \leq k$ and have the same phases in $\operatorname{gap}_{\mathrm{c}} . \quad c \in\left[\left[\varphi_{\text {conc }}\right]\right]$ ensures that for each $i: 1 \leq i \leq k, \operatorname{gap}_{\varphi}$ can be constrained so that $t_{\varphi}$ and $u_{\varphi}$ are registered with each $p_{i_{\varphi}}$ in gap $p_{\varphi}$ and have the same phases. Hence, the meet of the signal and wait gaps of $t_{\varphi}$ and $u_{\varphi}$ in $g a p_{\varphi}$ is not empty. This meet will actually be the phase of $t_{\varphi}$ on phasers $p_{i_{\varphi}}$ for $i: 1 \leq i \leq k$. phase ${ }_{\mathrm{c}}$ is then obtained from phase ${ }_{\mathrm{c}}$ by removing the phases of $u_{\mathrm{c}} \cdot g a p_{\varphi}^{\prime}$ is also obtained from $g a p_{\varphi}$ by removing $u_{\varphi}$. The task and phaser mappings $\kappa^{\prime}=\kappa \backslash\{u\}$ and $\pi$ witness that $\varphi^{\prime}$ denotes $\mathrm{c}^{\prime}$.

We define $\mathrm{c}_{0} \underset{\mathcal{T}}{\stackrel{\text { stmt }}{\longrightarrow}} c_{n}$ to mean a sequence $\mathrm{c}_{0} \underset{t_{1}}{\stackrel{\text { stmt }}{\longrightarrow}} \mathrm{c}_{1} \ldots \mathrm{c}_{n-1} \underset{t_{n}}{\stackrel{\text { stmt }}{\longrightarrow}} \mathrm{c}_{n}$ where $\mathcal{T}=\left\{t_{1}, \ldots t_{n}\right\}$ are the tasks in $\mathrm{c}_{0}$. Observe that this is in the transitive closure of $\stackrel{\text { stmt }}{\longrightarrow}$.

Theorem 15. Each predecessor computation rule $\stackrel{\text { stmt }}{\longrightarrow}$ in Figures 5.9 and 5.8 except for the rule newPhaser is complete with respect to the semantic rules of those in Figure 5.2 and $\underset{u}{\stackrel{\text { stmt }}{\longrightarrow}}$ defined above. The rule newPhaser is complete only when the task main executes it.

Proof. Assume $\varphi \underset{t_{\varphi}}{\stackrel{\text { stmt }}{\longrightarrow}} \varphi^{\prime}$ according to Figures 5.9 and 5.8. For any configuration $c^{\prime}=\left(\mathcal{T}_{\mathrm{c}^{\prime}}, \mathcal{P}_{\mathrm{c}^{\prime}}, 6 v_{\mathrm{c}^{\prime}}, p c_{\mathrm{c}^{\prime}}\right.$, phase $\left.{ }_{\mathrm{c}^{\prime}}\right)$ where $\mathrm{c}^{\prime} \in\left[\left[\varphi^{\prime}\right]\right]$, we exhibit a configuration $c=\left(\mathcal{T}_{\mathrm{c}}, \mathcal{P}_{\mathrm{c}}, 6 v_{\mathrm{c}}, p \mathcal{c}_{\mathrm{c}}\right.$, phase $\left._{\mathrm{c}}\right)$ such that $\mathrm{c}^{\prime} \underset{\kappa^{-1}\left(t_{\varphi}\right)}{\stackrel{\text { stmt }}{\longrightarrow}} \mathrm{c}$ and $\mathrm{c} \in \llbracket[\varphi]$. Note that any predecessor computation rule starts by concretizing $\varphi$ to some concrete constraint $\varphi_{\text {conc }}=\left(\mathcal{T}_{\varphi}, \mathcal{P}_{\varphi}, b v_{\varphi}, p c_{\varphi}, \operatorname{gap}_{\varphi}, \operatorname{egap}_{\varphi}\right)$. Then, the predecessor $\varphi^{\prime}$ is obtained from the $\varphi_{\text {conc }}$. Lemmas 14 and 15 ensure $\varphi \sqsubseteq \varphi_{\text {conc }}$ for any concrete constraint $\varphi_{\text {conc }}$. Hence, for every rule we show $c \in\left[\left[\varphi_{\text {conc }}\right]\right]$. This implies $c \in[[\varphi]]$.

Assume $\kappa$ and $\pi$ witness $c^{\prime} \in\left[\left[\varphi^{\prime}\right]\right]$. We show that using $\kappa^{-1}\left(t_{\varphi}\right)$ for $c^{\prime} \underset{\kappa^{-1}\left(t_{\varphi}\right)}{\stackrel{\text { stmt }}{\longrightarrow}}$ c generates the desired configuration c. The intuition is that if all tasks in $\mathcal{T}_{\mathrm{c}^{\prime}}$ that are associated to $t_{\varphi}$ by $\kappa$ execute stmt, we obtain a configuration $c$ that is denoted by some concrete constraint $\varphi_{\text {conc }}$, hence, denoted by $\varphi$.

newPhaser. This rule is only complete when $\kappa^{-1}\left(t_{\varphi}\right)$ is a singleton. To simplify the presentation, we show completeness when main executes newPhaser, because then we are sure $\kappa^{-1}\left(t_{\varphi}\right)$ is a singleton. Assume $t_{\varphi}$ is the task main in some $\varphi$ and $\varphi \frac{\text { ph:=newPhaser }()}{t_{\varphi}} \varphi^{\prime}$. Assume also $p_{\varphi}$ is the new phaser id. Let $p_{\mathrm{c}} \notin \mathcal{P}_{\mathrm{c}^{\prime}}$ and $\mathcal{P}_{\mathrm{c}}=\mathcal{P}_{\mathrm{c}^{\prime}} \cup\left\{p_{\mathrm{c}}\right\}$. phase ${ }_{\mathrm{c}}$ is obtained from phase ${ }_{\mathrm{c}^{\prime}}$ by assigning phase ${ }_{\mathrm{c}}\left(t_{\mathrm{c}}\right)\left(p_{\mathrm{c}}\right)=(\mathrm{ph},(0,0))$ where $t_{\mathrm{c}}$ is the task main in $\mathrm{c}^{\prime}$. Since $(0,0) \vDash \operatorname{gap}_{\varphi}\left(t_{\varphi}\right)\left(p_{\varphi}\right), t_{\mathrm{c}}$ can again be associated with $t_{\varphi}$ in $\varphi_{\text {conc }}$ 
and phase ${ }_{\mathrm{c}}\left(u_{\mathrm{c}}\right)\left(p_{\mathrm{c}}\right)=\left(-\right.$, nreg) for all other tasks $u_{\mathrm{c}} \in \mathcal{T}_{\mathrm{c}}^{\prime} \backslash\left\{t_{\mathrm{c}}\right\}$. Moreover, phase ${ }_{\varphi}\left(u_{\varphi}\right)\left(p_{\varphi}\right)=(-$, nreg $)$ for all tasks $u_{\varphi} \in \mathcal{T}_{\varphi}^{\prime} \backslash\left\{t_{c}\right\}$. Hence, for $\mathrm{c}=\left(\mathcal{T}_{\mathrm{c}}, \mathcal{P}_{\mathrm{c}}, 6 v_{\mathrm{c}}, p \mathcal{c}_{\mathrm{c}}\right.$, phase $\left._{\mathrm{c}}\right)$, we have $\mathrm{c}^{\prime} \frac{\text { ph:=newPhaser }()}{t_{\mathrm{c}}} \mathrm{c}$ and the task and phaser mappings $\kappa$ and $\pi^{\prime}=\pi \cup\left\{p_{\mathrm{c}} \rightarrow p_{\varphi}\right\}$ witness $\mathrm{c} \in\left[\left[\varphi_{\text {conc }}\right]\right]$.

signal. Assume $\varphi \frac{\text { ph.signal }()}{t_{\varphi}} \varphi^{\prime}$ for some $\varphi$. By definition of $c^{\prime} \in \llbracket\left[\varphi^{\prime}\right]$, there exists $p_{\mathrm{c}}$ such that $\pi\left(p_{\mathrm{c}}\right)=p_{\varphi}$ and all tasks in $\kappa^{-1}\left(t_{\varphi}\right)$ are associated with $t_{\varphi}$ by $\kappa . c^{\prime}$ is denoted by $\varphi^{\prime}$ and is obtained via signal I or signal II. There is therefore a level lev $\geq 0$ which shows that the signal and wait phases of the tasks registered in $c^{\prime}$ respect the phase bounds in $\varphi^{\prime}$. We can show that lev +1 captures that the signal and wait phases of the tasks registered in c respect the phase bounds in $\varphi$ if $\varphi^{\prime}$ is obtained via signal I, and lev shows that the signal and wait phases of the tasks registered in $c$ respect the phase bounds in $\varphi$ if $\varphi^{\prime}$ is obtained via signal II.

In both cases, the task and phaser mappings $\kappa$ and $\pi$ should be used for the denotation.

wait. Assume $\varphi \stackrel{\text { ph.wait }()}{t_{\varphi}} \varphi^{\prime}$ for some $\varphi$. By definition of $c^{\prime} \in\left[\left[\varphi^{\prime}\right]\right]$, there exists $p_{\mathrm{c}}$ such that $\pi\left(p_{\mathrm{c}}\right)=p_{\varphi}$ and each task $t \in \kappa^{-1}\left(t_{\varphi}\right)$ is associated with $t_{\varphi}$ by $\kappa$. If $l e v$ witnesses $c^{\prime} \in\left[\left[\varphi^{\prime}\right]\right]$, then the same level witnesses $c \in[[\varphi]]$ with the task and phaser mappings $\kappa$ and $\pi$.

drop. Assume $\varphi \stackrel{\text { ph.drop }()}{\underset{t_{\varphi}}{\longrightarrow}} \varphi^{\prime}$ for some $\varphi$. By definition of $c^{\prime} \in\left[\left[\varphi^{\prime}\right]\right]$, there exists $p_{\mathrm{c}}$ such that $\pi\left(p_{\mathrm{c}}\right)=p_{\varphi}$ and each task $t \in \kappa^{-1}\left(t_{\varphi}\right)$ is associated with $t_{\varphi}$ by $\kappa$. phase ${ }_{c}$ is obtained from prase $_{c^{\prime}}$ by 1 ) not modifying the phases of the tasks that are not associated with $\left.t_{\varphi}, 2\right)$ assigning phase ${ }_{\mathrm{c}}(t)\left(p_{\mathrm{c}}\right)=(-, \mathrm{nreg})$ for each $t \in \kappa^{-1}\left(t_{\varphi}\right)$. A level lev $\geq 0$ witnesses these tasks respect the phase bounds in $\varphi^{\prime}$ (which is obtained for a certain $\delta$ ). We can show lev- $\delta$ witnesses

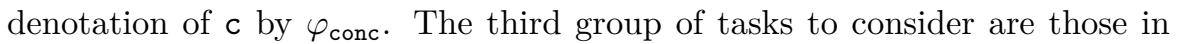
$\mathcal{T}_{\mathrm{c}} \backslash \kappa^{-1}\left(t_{\varphi}\right)$ which are registered with $p_{\mathrm{c}}$. The phases for these tasks are not modified. Hence, the task and phaser mappings $\kappa$ and $\pi$ witness $c \in\left[\left[\varphi_{\text {conc }}\right]\right]$.

exit. This proof is very similar to the proof of completeness of drop. The only difference is that the proof of drop needs to be extended by iterating through all phasers dropped in $c^{\prime}$. The mappings $\kappa^{\prime}=\kappa \backslash \kappa^{-1}\left(t_{\varphi}\right)$ and $\pi$ witness $c \in\left[\left[\varphi_{\text {conc }}\right]\right]$.

asynch. Assume $\varphi \underset{\text { asynch }\left(\operatorname{task}_{1} \mathrm{ph}_{1}, \ldots \mathrm{ph}_{\mathrm{k}}\right)\{\mathrm{s}\}}{t_{\varphi}} \varphi^{\prime}$ for some $\varphi$. Let $u_{\varphi}$ be the newly spawned task, and $p_{1_{\varphi}}, \ldots p_{k_{\varphi}}$ be the phasers passed to $u_{\varphi}$. By definition of $c^{\prime} \in\left[\left[\varphi^{\prime}\right]\right]$, there exist $p_{1_{c}}, \ldots p_{k_{c}}$ that $\pi\left(p_{i_{c}}\right)=p_{i_{\varphi}}$ for each $i: 1 \leq i \leq k$ 
and $\kappa$ associates each task $t \in \kappa^{-1}\left(t_{\varphi}\right)$ to $t_{\varphi}$, and each task $u_{t}$ spawned by $t$ to $u_{\varphi}$. We obtain phase from phase ${ }_{c^{\prime}}$ by copying phase ${ }_{c^{\prime}}(t)\left(p_{i_{c}}\right)$ to phase ${ }_{\mathrm{c}}\left(u_{t}\right)\left(p_{i_{c}}\right)$ for each $t \in \kappa^{-1}\left(t_{\varphi}\right)$ and $i: 1 \leq i \leq k$. Therefore, each $t \in \kappa^{-1}\left(t_{\varphi}\right)$ and $u_{t}$ can respectively be associated to $t_{\varphi}$ and $u_{\varphi}$ in $\varphi$. As a result, for $\mathrm{c}=\left(\mathcal{T}_{\mathrm{c}}, \mathcal{P}_{\mathrm{c}^{\prime}}, b v_{\mathrm{c}^{\prime}}, p c_{\mathrm{c}}\right.$, phase $\left.\mathrm{c}_{\mathrm{c}}\right)$, we have $\mathrm{c}^{\prime} \frac{\operatorname{asynch}\left(\operatorname{task}, \mathrm{ph}_{1}, \ldots \mathrm{ph}_{\mathrm{k}}\right)\{\mathrm{s}\}}{\kappa^{-1}\left(t_{\varphi}\right)} \mathrm{c}$ and the task and phaser mappings $\kappa^{\prime}=\kappa \cup\left\{t \rightarrow t_{\varphi} \mid t \in \kappa^{-1}\left(t_{\varphi}\right)\right\} \cup\left\{u_{t} \rightarrow u_{\mathrm{c}} \mid t \in \kappa^{-1}\left(t_{\varphi}\right)\right\}$ and $\pi$ witness $c \in\left[\left[\varphi_{\text {conc }}\right]\right]$.

\subsubsection{Verification Procedure}

The procedure "paramPhaserExplore" $\left(\Phi_{b a d}, \mathrm{c}_{s t r t}, \mathrm{~S}, \rightarrow\right.$, ᄃ) makes use of a predecessor computation (line 7 ) that results, for a constraint $\varphi$ and a statement stmt, in a finite set pre stmt $=\left\{\varphi^{\prime} \mid \varphi \stackrel{\text { stmt }}{\longrightarrow} \varphi^{\prime}\right\}$ with task $t$ which is either a task in $\varphi$ or is a fresh task. Observe that renaming tasks and phasers in $\varphi$ does not change the denotation. Therefore, we keep only one representa-

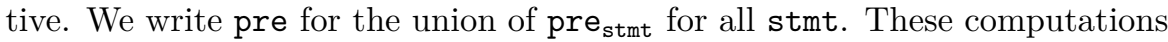
are described in Figures 5.8 and 5.9. Intuitively, the program statement for which the predecessors set is being computed can be executed by a task that is either captured by the constraint $\varphi=(\mathcal{T}, \mathcal{P}, 6 v, p c, p v, \gamma)$ explicitly (i.e., $t \in \mathcal{T}$ ) or implicitly (i.e., $t \notin \mathcal{T}$ but satisfies the environment gaps). For all but newPhaser and atomic statements (i.e., ph.next ()$\{\mathbf{s}\})$, the set pre stmt is exact in the sense that $\left\{c^{\prime} \mid c \in[\varphi]\right]$ and $\left.c^{\prime} \stackrel{\text { stmt }}{\longrightarrow} c\right\} \subseteq \cup_{\varphi^{\prime} \in \text { pre }_{\text {stmt }}}\left[\left[\varphi^{\prime}\right] \subseteq\right.$ $\left\{c^{\prime} \mid c \in[[\varphi]]\right.$ and $\left.c^{\prime} \stackrel{\text { stmt }}{\longrightarrow} c\right\}$. Intuitively, the atomic ph.next ()$\{$ stmt $\}$ statement can encode a testAndSet operation. Such an operation can be made to be carried by exactly one task. Our representation allows for more tasks (and larger gaps), but the additional tasks may not be able to carry the atomic operation. In fact, we show in Section 5.6 that allowing atomic instructions results in the undecidability of the problems addressed by Theorems 16 and 17. The proposed newPhaser predecessor computation is exact when only one task carries it (e.g., main). Otherwise, assume a task $t$ in the constraint creates a new phaser $p$. If in a configuration each task that is mapped (by a surjection) to $t$ creates a phaser, several phasers are created and it is not possible to map all of them by a bijection to $p$. Without this restriction the predecessor computation for newPhaser is sound but not complete.

Using the soundness of $\sqsubseteq$ (Lemma 5) and the soundness of predecessor computation (Lemma 14) we can show soundness of "paramPhaserExplore".

Lemma 21 (Soundness). If "paramPhaserExplore" $\left(\Phi_{b a d}, \mathrm{c}_{s t r t}, \mathrm{~S}, \rightarrow\right.$, ᄃ) returns unreachable, then $\mathrm{c}_{\text {strt }} \stackrel{*}{\rightarrow}\left[\left[\Phi_{\text {bad }}\right]\right]$. 


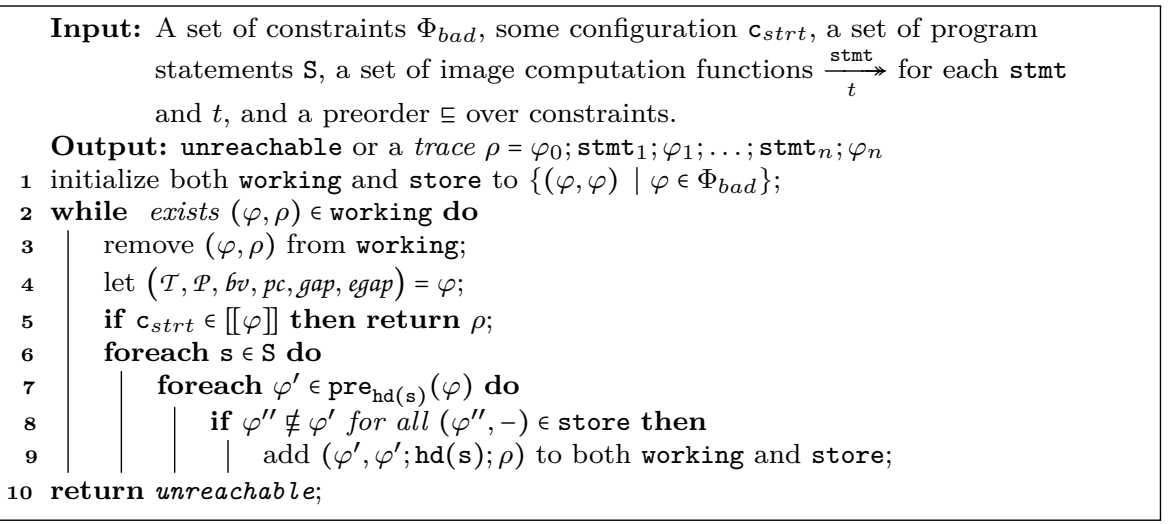

Procedure paramPhaserExplore $\left(\Phi_{b a d}, \mathrm{c}_{s t r t}, \mathrm{~S}, \rightarrow\right.$, 드). A working-list procedure for checking reachability for phaser programs. The procedure checks whether some configuration $\mathrm{c}_{\text {strt }}$ is reachable from one of the

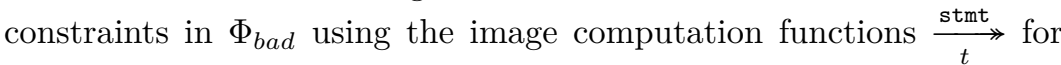
each stmt and $t$, and the preorder $\subseteq$ on the constraints.

Proof. The procedure "paramPhaserExplore" $\left(\Phi_{b a d}, \mathrm{c}_{s t r t}, \mathrm{~S}, \rightarrow, \sqsubseteq\right)$ is an instantiation of the procedure "explore" in Section 3.3. The function isTarget in "explore" is instantiated with the denotation function for configurations and constraints. By soundness of both $\rightarrow$ (Theorem 14) and the procedure "explore" (Theorem 4), the procedure "paramPhaserExplore" $\left(\Phi_{\text {bad }}, \mathrm{c}_{s t r t}, \mathrm{~S}, \rightarrow\right.$, 드 is sound.

Lemma 22 (Relative completeness). If the procedure "paramPhaserExplore" $\left(\Phi_{\text {bad }}, \mathrm{c}_{\text {strt }}, \mathrm{S}, \rightarrow\right.$, ㄷ) returns a trace $\varphi_{0} ;$ stmt $_{0} ; \ldots ; \varphi_{n-1} ;$ stmt $_{n-1}, \varphi_{n}$ in which only the task main has executed newPhaser, there are $\mathrm{c}_{0}, \ldots \mathrm{c}_{n}$ with $\mathrm{c}_{0}=\mathrm{c}_{\text {strt }}, \mathrm{c}_{n} \in\left[\left[\Phi_{\text {bad }}\right]\right]$ and $\mathrm{c}_{i-1} \stackrel{\text { stmt }_{i-1}}{\longrightarrow} \mathrm{c}_{i}$ for $i: 1<i \leq n$.

Proof. The procedure "paramPhaserExplore" $\left(\Phi_{b a d}, \mathrm{c}_{s t r t}, \mathrm{~S}, \rightarrow, \sqsubseteq\right)$ is an instantiation of the procedure "explore" in Section 3.3. The function isTarget in "explore" is instantiated with the denotation function for configurations and constraints, which is by definition complete. By relative completeness of $\rightarrow$ (Theorem 15) and completeness of the procedure "explore" (Theorem 5), the procedure "paramPhaserExplore" $\left(\Phi_{b a d}, \mathrm{c}_{s t r t}, \mathrm{~S}, \rightarrow\right.$, ㄷ) is complete relative to the runs is which only the task main is allowed to execute newPhaser.

We can also show that the procedure terminates if we only manipulate $K$-bounded-dimension and $B$-good constraints.

Lemma 23 (Termination). "paramPhaserExplore" $\left(\Phi_{b a d}, \mathrm{c}_{s t r t}, \mathrm{~S}, \rightarrow\right.$, ᄃ) terminates if there are $K, B \in \mathbb{N}$ such that all constraints in store are $K$ dimension-bounded and B-good. 
Proof. If all constraints are $K$-dimension-bounded and $B$-good for $K, B \in \mathbb{N}$, then according to Theorem 13 the preorder $ᄃ$ is a $\mathcal{W} \mathcal{Q O} . \rightarrow$, ᄃ, and $\vDash$ are effective by definition. Hence, by Theorem 6 we conclude "paramPhaserExplore" $\left(\Phi_{b a d}, \mathrm{c}_{\text {strt }}, \mathrm{S}, \rightarrow\right.$, ㄷ) terminates.

Theorem 16. Control reachability for non-atomic phaser programs that generate a finite number of phasers is decidable if only main is allowed to create phasers.

Proof. $K$-boundedness is ensured by systematically dropping the generated constraints in the backward procedure that violate $K$-dimension-boundedness (as none of the denoted configurations are reachable). Also, the set of the target constraints is free (since we are checking control reachability) and this is preserved by the pre computation in Figure 5.6, 5.8, and 5.9. Finally, Lemmas 21,22 , and 23 ensure soundness, relative completeness, and termination.

Theorem 17. Plain reachability is decidable for non-atomic phaser programs that generate at most $K$ phasers with $B$-bounded gaps for each phaser if only main is allowed to create phasers.

Proof. In the backward procedure, systematically dropping the generated constraints that require more than $K$ phasers or gap-values larger than $B$ for some phaser gaps (as none of the denoted configurations are reachable) ensures $K$ dimension-boundedness and $B$-goodness. Finally, Lemmas 21, 22, and 23 ensure soundness, relative completeness, and termination.

\subsection{Limitations of Deciding Reachability}

Assume a program $\operatorname{Pr}=(\mathrm{B}, \mathrm{P}, \mathrm{Ts})$ and a configuration $\mathrm{c}_{\text {strt }}$. We show a number of parameterized reachability problems to be undecidable. First, we address checking control reachability when restricting to configurations with at most $K$ task-referenced phasers. We call this $K$-control-reachability.

Definition 10 (K-control-reachability). Given a program $\operatorname{Pr}, \quad$ a configuration $\mathrm{c}_{\text {strt }}$, and a partial control configuration $\mathrm{c}_{\text {trgt }}$, we write $\operatorname{reach}_{K}\left(\operatorname{Pr}, \mathrm{c}_{\text {strt }}, \mathrm{c}_{\text {trgt }}\right)$, and say $\mathrm{c}_{\text {trgt }}$ is $K$-control-reachable, to mean there are $n+1$ configurations $\left(\mathrm{c}_{i}\right)_{i: 0 \leq i \leq n}$, each with at most $K$ reachable phasers (i.e., phasers referenced by at least a task variable) such that $\mathrm{c}_{\text {strt }}=\mathrm{c}_{0}$ and $\mathrm{c}_{i} \rightarrow \mathrm{c}_{i+1}$ for $i: 0 \leq i<n$ with $\mathrm{c}_{n}$ equivalent to a configuration that includes $\mathrm{c}_{\text {trgt }}$.

Theorem 18. K-control-reachability is undecidable in general. 

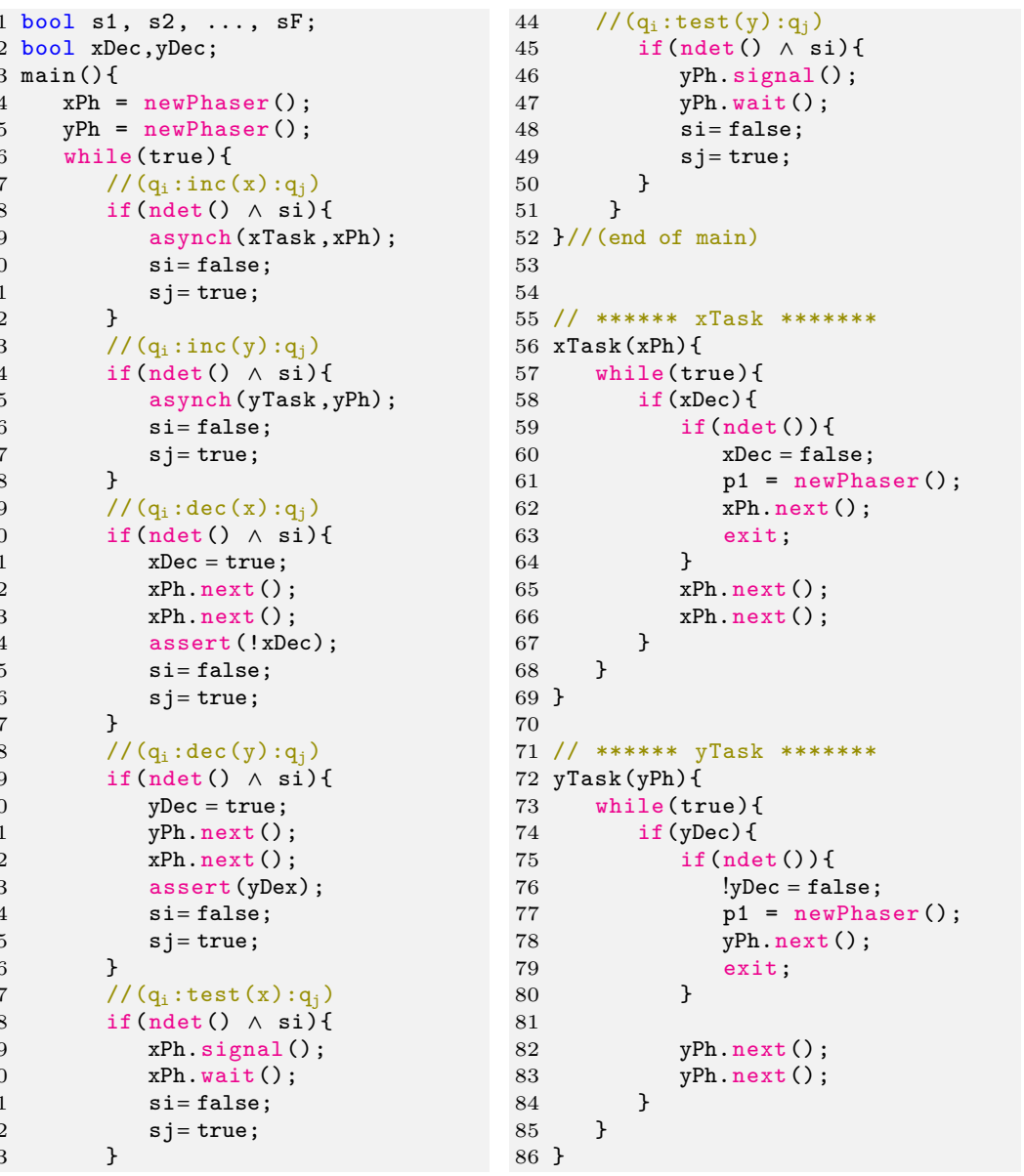

Figure 5.10: Encoding a Minsky machine with the two counters $\{x, y\}$ for the proof of Theorem 18. The value of counter $x$ is represented by the number of instances of xTask tasks registered to phaser $\mathrm{xPh}$. The construction ensures that runs trying to decrement a counter by more than one will result in configurations with a larger number of phasers than $K$. 


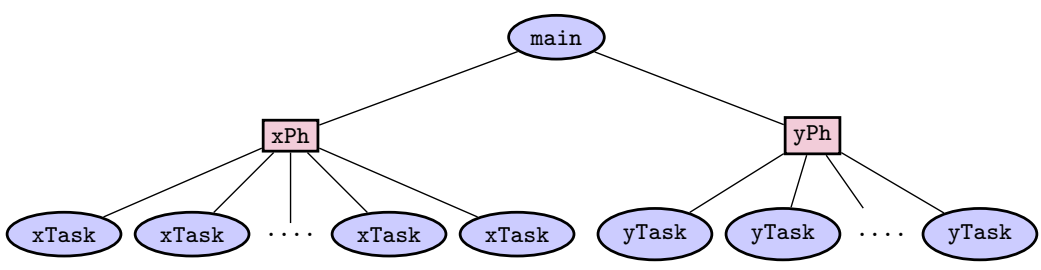

Figure 5.11: A possible configuration for the phaser program in proof of Theorem 18 that consists of two groups of tasks of types xTask and yTask. The number of tasks in each cluster represents the value of the corresponding counter, namely, $x$ and $y$

Proof. We encode the state reachability of an arbitrary Minsky machine with counters $x$ and $y$ using $K$-control-reachability of a suitable phaser program. We assume $K=3$. The program depicted in Figure 5.10 has three tasks: main, xTask, and yTask. Machine states $q \in \mathcal{Q}$ are captured with shared Boolean variables and counter values with the number of xTask registered with phaser $\mathrm{xPh}$ for counter $x$ (respectively, the number of yTask registered with phaser $\mathrm{yPh}$ for the counter $y$ ). A sample configuration of this program is illustrated in Figure 5.11. We reason about the correctness of our encoding for counter $x$. The reasoning for counter $y$ would be similar.

Incrementing the counter $x$ is encoded by spawning an instance of xTask and registering it with $\mathrm{xPh}$ (line 9 of Figure 5.10).

Test for zero is performed by a signal and a wait on $\mathrm{xPh}$ (lines 39 and 40).

Decrementing the counter $x$ involves asking an xTask, via shared variables, to exit (hence, to deregister from $\mathrm{xPh}$ ). However, more than one task might participate in the decrement operation. For this reason, each participating task creates a new phaser. In this way, if more than one xTask participates in the decrement, then the number of reachable phasers of an intermediary configuration will be at least 4 , and will therefore be discarded because $4>K$. A possible such configuration is depicted in Figure 5.12.

First, the task main sets xDec to true (line 21). This will satisfy the ifcondition at line 58 of any xTask. These tasks non-deterministically choose whether or not to participate in the decrement by the if-condition at line 59 . main performs two next operations on the phaser $\mathrm{xPh}$. Those xTask:s that do not participate will perform two next operations at lines 65 and 66 in order to not block the ones that participate. If no such task participates, the assertion at line 24 would be violated. 


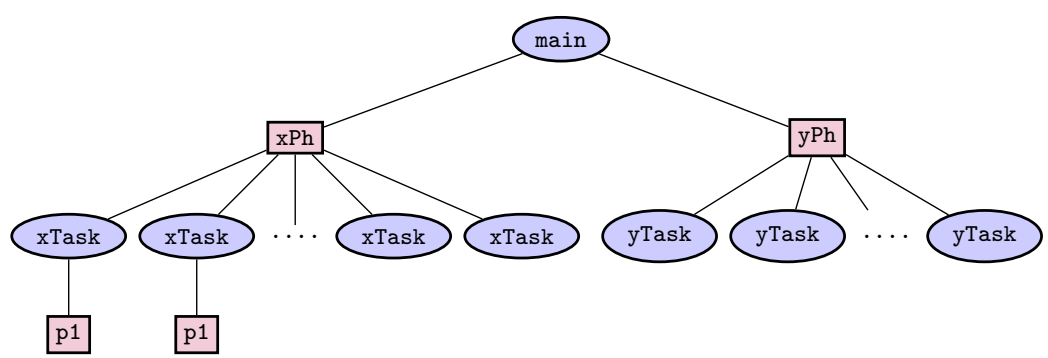

Figure 5.12: A possible configuration for the phaser program in proof of Theorem 18 consisting of two groups of tasks of types xTask and yTask. This configuration is the result of two xTask tasks trying to decrement the counter $x$ which violates $K$-control boundedness for $K=3$.

Any participating xTask has to create a phaser $\mathrm{p} 1$ before being able to execute xPh.next() at line 62. Thereafter, xTask can exit which enables main to not block at xPh.next() (line 23).

The above encoding ensures that the two phasers $\mathrm{xPh}$ and $\mathrm{yPh}$ appear in all configurations. Moreover, a decrement operation will add one extra phaser per participating xTask. Hence, if more than one such task participates, $K$ will exceed 3, and those configurations will be discarded. As a result, from the initial configuration $\mathrm{c}_{\text {init }}$, the phaser program will reach a configuration in which $\mathrm{sF}=$ true via configurations that have at most three reachable phasers, iff the counter machine reaches a configuration with the target state $\mathbf{q}_{F}$.

Theorem 19. The control reachability of phaser programs that generate a finite number of phasers is undecidable if atomic statements are allowed.

Proof. We encode the state reachability problem of an arbitrary Minsky machine with counters $x$ and $y$ using a phaser program with atomic statements. The phaser program depicted in Figure 5.13 should have three tasks: main, xTask and yTask. yTask is similar to xTask. The idea is to associate a phaser $\mathrm{xPh}$ to counter $x$ (respectively, yPh to counter $y$ ). We introduce in the following the encoding of the Minsky machine operations on the counter $x$ in the phaser program. Counter $y$ is very similar to that.

Increment. main spawns a task xTask and registers it with xPh (line 10).

Decrement. First, main sets xDec to true and performs next with atomic statement on $\mathrm{xPh}$ (lines 18 and 19). One of the existing xTask:s will execute the next atomic statement (lines 47 and 48) and reset $x D e c$ and exit. Then, 


\begin{tabular}{|c|c|c|c|c|}
\hline & \multicolumn{4}{|c|}{ Arbitrary numbers of tasks } \\
\hline & \multicolumn{2}{|c|}{ Finite dimension } & $K$-reachability & Arbitrary dimension \\
\hline $\begin{array}{l}\text { Bounded } \\
\text { gaps }\end{array}$ & $\begin{array}{l}\text { ctrl atomic } \boldsymbol{X} \\
(\text { Theorem } 19)\end{array}$ & $\begin{array}{c}\text { plain non-atomic } \checkmark \\
\text { (Theorem 17) }\end{array}$ & \multirow{2}{*}{$\begin{array}{l}\text { ctrl non-atomic } \boldsymbol{X} \\
(\text { Theorem 18) }\end{array}$} & \multirow{2}{*}{$\begin{array}{c}\text { ctrl non-atomic } \boldsymbol{X} \\
(\text { Theorem 12) }\end{array}$} \\
\hline $\begin{array}{l}\text { Arbitrary } \\
\text { gaps }\end{array}$ & $\begin{array}{l}\text { ctrl non-atomic } \checkmark \\
\text { (Theorem 16) }\end{array}$ & $\begin{array}{c}\text { plain non-atomic } \boldsymbol{X} \\
(\text { From }[62])\end{array}$ & & \\
\hline
\end{tabular}

Table 5.1: Findings summary: ctrl stands for control reachability and plain for plain reachability; atomic stands for allowing the ph.next()\{stmt\} atomic instruction and non-atomic for forbidding it (resulting in non-atomic programs). Recall that the dimension of a phaser program is the number of dynamically generated phasers. Our reachability procedure is sound for all problems discussed in the table. Problems that are guaranteed to terminate and are complete relative to the runs in which only main creates new phasers are marked with $\boldsymbol{}$. The undecidable problems are marked with $\boldsymbol{x}$.

programs when only distinguished tasks such as main create phasers. The procedure can be used for answering both control and plain reachability problems. We summarize our findings in Table 5.1. The procedure is guaranteed to terminate, even for programs that may generate arbitrarily many tasks but finitely many phasers, when checking control reachability or when checking plain reachability with bounded gaps. These results were obtained using a non-trivial symbolic representation for which termination had required showing an $\exists_{\forall}$ preorder on multisets on gaps on natural numbers to be a $\mathcal{W} \mathcal{Q O}$.

We believe our general decidability results are useful for reasoning about synchronization constructs other than phasers. For instance, one phaser with bounded gaps (in fact, one) can capture a traditional static barrier. Similarly, one phaser with one producer and arbitrarily many consumers can be used to capture futures where a "get" instruction can be modeled with a wait. We believe our negative results can also be used. For instance, atomic instructions can be modeled using testAndSet operations and may result in the undecidability of the reachability problem. This suggests that more general applications of the work are to be investigated. 



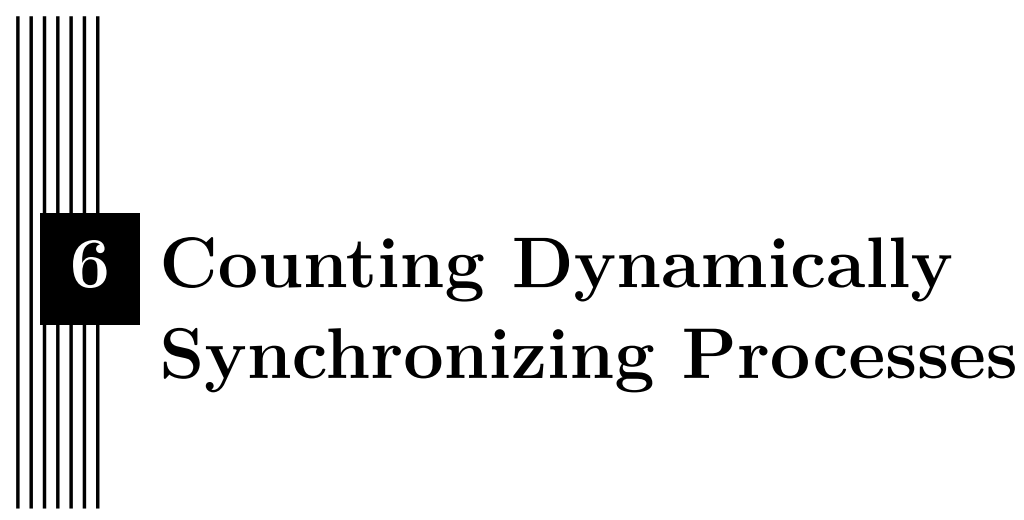

In this chapter, we address the problem of automatically establishing correctness for parameterized programs that generate an arbitrary number of concurrent processes and manipulate variables ranging over an infinite domain. The programs we consider can make use of shared variables to count and synchronize the spawned processes. This allows them to implement intricate synchronization mechanisms, such as barriers. For this purpose, we introduce counting predicates that mix counters referring to the number of processes satisfying certain properties and variables directly manipulated by the concurrent processes. We then combine existing works on counter, predicate, and constrained monotonic abstraction and build a nested counterexample-based refinement scheme for establishing correctness (expressed as non-reachability of configurations that satisfy counting predicates). We have implemented a tool (PACMAN, for predicated constrained monotonic abstraction $\underline{\text { m }}$ ) and used it to perform parameterized verification on several programs whose correctness crucially depends on precisely capturing the number of processes synchronizing through the use of shared variables.

\subsection{Introduction}

We consider programs that spawn arbitrarily many concurrent processes that use shared integer variables for counting the number of processes at different stages of their computations. Such counters might be used to implement a synchronization mechanism among processes. Correctness is stated in terms 
of safety properties expressed using counting predicates. Counting predicates make it possible to express statements about program variables and counters for the number of processes satisfying certain predicates on program variables. Such statements can capture both individual properties, such as assertion violations, and global properties, such as deadlocks or relations between the numbers of processes in certain states.

Handling programs with barriers of arbitrary sizes (i.e., the number of processes participating in the barrier is fixed but arbitrarily large) is not a trivial task even when all processes only manipulate Boolean variables. To enforce the correct behavior of a barrier, a verification procedure needs to capture relations between the number of processes satisfying certain properties, for instance, that all processes are waiting at the barrier before any of them is allowed to cross it. This amounts to testing that the number of processes at specific locations is zero. Capturing increments and decrements of counters in a given counter machine can be done by having counters that track the number of processes satisfying predicates such as being at some program location, and adding the possibility to have a single process change location. In this case, one can argue that checking violations of program assertions is tantamount to checking state reachability of a counter machine. Therefore, no sound verification technique can be complete for such systems, since the problem is undecidable (Theorem 1).

Our approach to getting around this problem builds on the following observation. In case there are no tests for the number of processes satisfying certain properties (e.g., being in specific program locations), symmetric Boolean concurrent programs can be exactly encoded as monotonic counter machines, i.e., essentially vector addition systems (VASs). For such systems, state reachability can be decided using the backward reachability analysis that was introduced in Section 3.3. This approach is exact because of the monotonicity of the induced transition system (more processes can fire more transitions since there are no tests on the numbers of processes). Termination is guaranteed by the well-quasi-ordering of the component-wise ordering on the natural numbers. The induced transition system is not any more monotonic in the presence of tests on the number of processes. In this case we apply monotonic abstraction [4] as introduced in Section 3.4. The idea in monotonic abstraction is to modify the semantics of the entailed tests (e.g., zero tests for barriers), so that processes that do not satisfy the tests are removed (e.g., zero tests are replaced by resets). This results in a monotonic over-approximation of the original transition system in which spurious traces are possible. Indeed, having more processes while respecting important relations between their numbers and certain variables in the original programs does not necessarily allow to fire more transitions (which is what abstracted programs do in such approaches).

To sum up, our approach consists of combining two nested counterexample-guided abstraction refinement loops. Each loop operates 
at a different level of abstraction. We summarize our contributions in the following points.

1. We introduce and propose the use of counting predicates to express statements about program variables and the number of processes satisfying given predicates on program variables.

2. We implement an abstraction refinement loop (the outer loop in our approach) by leveraging existing symmetric predicate abstraction techniques. We encode the resulting Boolean programs in terms of counter machines where reachability of configurations satisfying a counting predicate formula is captured as a state reachability problem.

3. We explain how to strengthen the counter machine using counting invariants, i.e., counting predicates that hold on all runs. We automatically generate such invariants using classical thread-modular analysis techniques.

4. We leverage existing constrained monotonic abstraction techniques to implement the inner loop of our approach and address the counter machine state reachability problem.

5. We implement both loops, together with automatic counting invariants generation, in a prototype tool (PACMAN) that has allowed us to automatically establish or refute counting predicate formulas such as deadlock freedom and assertions. All programs we report on may generate arbitrarily many processes.

Related work. Several works consider parameterized verification for concurrent programs. See [130] for a good survey. We report on some relevant techniques. The works in $[81,6]$ explore finite instances and automatically check for cutoff conditions. Except for checking larger instances, however, there is no straightforward approach to refine the entailed abstractions. [7] strengthens the approach of [6], but cannot capture global properties that involve relations between the number of processes and program variables, as we do in this work. In [57], the authors target verification of Petri nets, which are inherently monotonic and generate invariants by weakening SMT formulas. Although we also target coverability, we do it for counter machines obtained by strengthening monotonic systems into non-monotonic ones. There is no straightforward approach to apply their method to the non-monotonic systems that we consider. The work in [85] gives a generalization of the IC3 algorithm and tries to build inductive invariants for well-structured transition systems. Adapting it to the non-monotonic systems that we work with is not trivial. Similar to [1], we combine auxiliary invariants obtained on specific variables to strengthen a reachability analysis. In [58], the authors propose an approach that involves synthesizing counters to build correctness proofs from program 
traces automatically. The approach repeatedly builds safe counting automata and establishes that their language includes traces of a program given as a control flow net. Such nets can model arbitrarily many processes sharing global variables. We can also handle local variables and automatically discover relevant predicates by nesting constrained monotonic abstraction loops within symmetric predicate abstraction loops. In [19], the authors present a highly optimized coverability checking approach for VASs with broadcasts. We need more than the coverability of monotonic systems. In [83], the authors adopt symbolic representations that can track inter-thread predicates. This yields a non-monotonic system, and the authors force monotonicity as in $[4,3]$. However, they do not explain how to refine the obtained decidable monotonic abstraction for an undecidable problem. In [16], the authors prove termination for depth-bounded systems by instrumenting a given over-approximation with counters and sending the numerical abstraction to existing termination provers. We automatically generate the abstractions on which we establish safety properties. In addition, and as stated earlier, over-approximating the concurrent programs we target with (monotonic) well-structured transition systems would result in spurious runs.

The closest works to ours are $[3,51,66]$. Monotonic abstraction in $[4,3]$ is not combined with predicate abstraction, nor does it explicitly target counting properties or dynamic barrier based synchronization. In [51], the authors propose a predicate abstraction framework for concurrent multi-threaded programs. As explained earlier, such abstractions cannot exclude behaviors forbidden by synchronization mechanisms such as barriers. In our work, we build on [51] in order to handle shared and local integer variables. The approach in [66] introduces an invariant synthesis method that uses horn constraints and automatically infers the counters that are required for program verification. In contrast, we use counters for all program locations. Although their approach outperforms ours because of maintaining fewer counters, it is not made clear how to automatically encode the input programs as horn clauses.

Outline. We start by illustrating our approach using an example in Section 6.2 and introduce some preliminaries in Section 6.3. We then define concurrent programs and describe our counting predicates in Section 6.4. Next, we explain the different phases of our nested loop in Section 6.5 and report on our experimental results in Section 6.6. We finally conclude in Section 6.7.

\subsection{Illustrative Example}

Consider the concurrent program listed in Figure 6.1. In this program, processes share four integer variables, namely arrived, all, read and cross. Variable all is initialized to 1 , and variables arrived, cross and read are initialized to 0 . A single process starts executing the program transitions. 


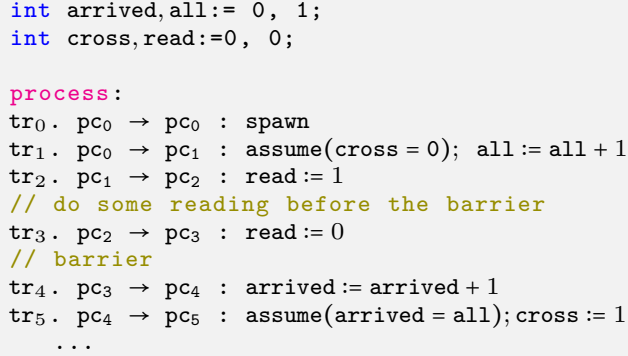

Figure 6.1: A sample parameterized concurrent program in which processes synchronize using shared variables. No matter how many processes are spawned, no process can be at $\mathrm{pc}_{5}$ when read $>0$.

Each transition may consist of several statements. Each statement is either a spawn, join, multi-assignment, or an assume(). Arbitrarily many processes get spawned at location $\mathrm{pc}_{0}$ by transition $t r_{0}$.

Each process executes $t r_{1}$ and increments all if cross is 0 , meaning that no process has crossed the barrier. Otherwise, no process can execute the transition $\operatorname{tr}_{1}$. It then sets and resets read. Intuitively, read is greater than zero when there is at least one process doing some reading before the barrier between transitions $t r_{2}$ and $t r_{3}$. Transitions $t r_{4}$ and $t r_{5}$ essentially implement a barrier in the sense that all processes must have reached $\mathrm{pc}_{4}$ in order for any of them to move to location $\mathrm{pc}_{5}$. The first process that crosses the barrier changes cross from 0 to 1 . As a result, no other process can take transition $\operatorname{tr}_{1}$ and start working. After the barrier, no process should be left behind. We capture this by asserting that no process at location $\mathrm{pc}_{5}$ should witness read $>0$. We write $@ \mathrm{pc}_{5}$ to mean the predicate satisfied by all processes at location $\mathrm{pc}_{5}$. A process that satisfies $@ \mathrm{pc}_{5} \wedge(\mathrm{read}>0)$ is at location $\mathrm{pc}_{5}$ and witnesses read $>0$. A configuration of the program satisfies the predicate $\left(@ \mathrm{pc}_{5} \wedge(\mathrm{read}>0)\right)^{\#} \geq 1$ if the number of such processes in the configuration is greater than or equal to one. We call such predicate a counting predicate (introduced in Sec 6.4). Counting predicates can be used to capture configurations that violate properties other than assertions, e.g., deadlock freedom.

The assertion $\left(@ \mathrm{pc}_{5} \wedge(\mathrm{read}>0)\right)^{\#} \geq 1$ is never violated under any run starting from a configuration in which a single process starts executing from location $\mathrm{pc}_{0}$. To establish this fact, any verification procedure needs to take into account the barrier at $\operatorname{tr}_{5}$ as well as the two sources of infiniteness, namely, the infinite domain of the shared and local variables and the number of processes that may participate in the run. Apart from [58], which cannot handle local variables, and [66], the closest works to ours deal with these two sources of infiniteness separately and cannot capture facts that relate them, 


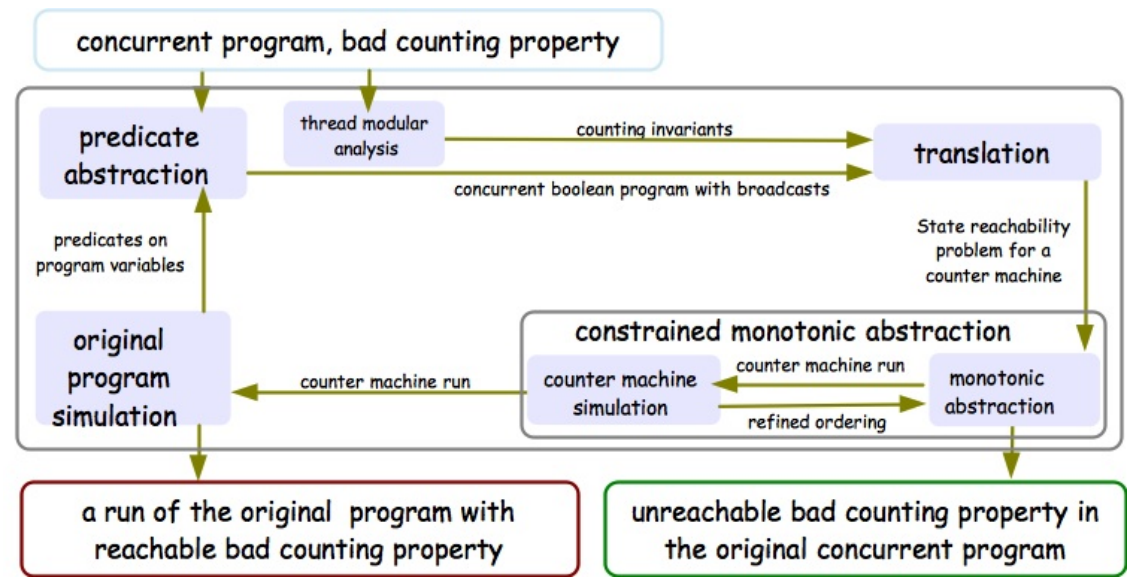

Figure 6.2: Predicated Constrained Monotonic Abstraction

namely, the values of the program variables and the number of generated processes.

Any sound analysis that does not take into account that all captures the number of processes at locations $\mathrm{pc}_{1}$ or later, and that arrived represents the number of processes at locations $\mathrm{pc}_{4}$ or later, will not be able to discard scenarios where a process executes read $:=1$ although another one is at $p c_{5}$. Such an analysis will therefore fail to show that read $=0$ each time a process is at $\mathrm{pc}_{5}$.

Our tool, which is called Predicated Constrained Monotonic Abstraction and is depicted in Figure 6.2, systematically leverages simple facts that relate numbers of processes to the variables manipulated in the program. This allows us to verify or refute safety properties (e.g., assertions and deadlock freedom) depending on complex behaviors induced by constructs, such as dynamic barriers. We illustrate our approach, which consists of two nested CEGAR loops in the remaining of this section using the example of Figure 6.1 .

From concurrent programs to Boolean concurrent programs. We build on the recent predicate abstraction techniques for concurrent programs. Such techniques first discard all variables and predicates and only keep the control flow. This leads to several counterexample-guided abstraction refinement steps (the outer CEGAR loop in Figure 6.2) that will result in the addition of new predicates. Our implementation automatically adds the predicates cross_leq_0, read_leq_0, arrived_leq_all and all_leq_arrived.

It is worth noticing that all variables of the obtained concurrent program are finite-state (in fact, Boolean). Hence, one would need a finite number of 
counters in order to faithfully capture the behavior of the abstracted program using counter abstraction.

From concurrent Boolean programs to counter machines. Given a concurrent Boolean program and a property to be checked, we generate a counter machine that essentially boils down to a vector addition system with transfers (with additional tests for global properties such as deadlockfreedom). Each counter in the machine counts the number of processes at some location with some specific value combination for the local variables. One state in the counter machine represents reaching a configuration that violates the property we want to check. The other states of the counter machines correspond to the possible combinations of the global variables. Such a machine cannot relate the number of processes in certain locations to the predicates that are valid at certain states (for instance, that all = arrived). These are essential for the verification of programs where counters are used to synchronize processes. In order to remedy this issue, we make use of counting invariants that relate program variables, all and arrived in the following invariants, to the number of processes at certain locations.

$$
\begin{aligned}
& \text { all }=\sum_{i \geq 1}\left(@ \mathrm{pc}_{\mathrm{i}}\right)^{\#} \\
& \text { arrived }=\sum_{i \geq 4}\left(@ \mathrm{pc}_{\mathrm{i}}\right)^{\#}
\end{aligned}
$$

We automatically generate such invariants using a simple thread modular analysis that tracks the number of processes satisfying some property.

Example 5 (Thread-modular analysis). To recover the two counting invariants above, we can perform a classical thread modular analysis [70] where we add a shared instrumentation variable $\mathrm{pc}_{\mathrm{i}}$ to track the number of processes at location $\mathrm{pc}_{\mathrm{i}}$ for $i: 0 \leq i \leq 6$. We use a suitable abstract numerical domain (in this case, polyhedral domain). For the program of Figure 6.1, we obtained the counting invariants mentioned earlier as well as other invariants such as $0 \leq$ all, $0 \leq$ arrived and arrived $\leq$ all that helped for pruning the state space (Section 6.6).

Given such counting invariants, we constrain the counter machine and generate a more precise machine that may not be a vector addition system anymore. We explain in Section 6.5 that the resulting state reachability problem is now undecidable in general.

Constrained monotonic abstraction. We monotonically abstract the resulting counter machine in order to answer the state reachability problem. Spurious traces are now possible. Essentially, monotonic abstraction closes the obtained sets of predecessor configurations upwards. This overapproximation might add larger configurations that did not belong to the set 
of predecessor configurations. Intuitively, the effect of monotonic abstraction "in forward" on the example of Figure 6.1 is that it "deletes" processes violating the constraint imposed by the barrier [4]. This example illustrates a situation where such approximations yield false positives. To see this, suppose two processes exist. The first process gets to $\mathrm{pc}_{4}$ and waits. The second process moves to $\mathrm{pc}_{2}$. Deleting the second process is allowed by the monotonic abstraction and opens the barrier for the first process. However, the assertion can now be violated because the deleted process did not have time to reset the variable read. Constrained monotonic abstraction eliminates spurious traces by refining the preorder used in monotonic abstraction. For the example of Figure 6.1 , if the number of processes at $\mathrm{pc}_{1}$ is zero, then closing upwards will not alter this fact. By doing so, the process deleted in forward at $\mathrm{pc}_{2}$ is not allowed to be there to start with, and the assertion is automatically established for any number of processes. The inner loop of our approach can automatically perform more elaborate refinements such as comparing the number of processes at different locations. Exact traces of the counter machine are sent to the next step and unreachability of the control location establishes the safety of the concurrent program.

Trace Simulation. Traces obtained in the counter machine reachability problem are real traces as far as the concurrent Boolean program is concerned. Those traces can be simulated on the original program to find new predicates (e.g., using Craig interpolation) and use them in the next iteration of the outer loop.

\subsection{Preliminaries}

We write $\mathbb{N}$ and $\mathbb{Z}$ to mean the sets of natural and integer values respectively. Given two natural numbers $i, j \in \mathbb{N}$, we use $[i, j]$ to denote the set $\{k \in \mathbb{N} \mid i \leq$ $k \leq j\}$. We let $\mathbb{B}=\{$ true, false $\}$ be the set of Boolean values.

We write $\mathrm{V}$ and $\mathrm{B}$ to respectively mean a set of integer and Boolean variables. We write $X$ to mean some set V or B. Similarly, we write $\mathrm{v}$ and $\mathrm{v}^{b}$ to respectively mean an integer or a Boolean variable. We also write $x$ to mean a variable of some type.

We write exprsOf(V) to mean the set of arithmetic expressions over the integer variables V. An arithmetic expression e (or expression for short) in $\operatorname{exprsOf(V)}$ is either an integer constant $\mathrm{k}$, an integer variable $\mathrm{v}$ in $\mathrm{V}$, or the sum or difference of two expressions as described below:

$$
\text { e }::=k|v|(e+e)|(e-e)| k e \quad v \in V
$$

We let $\sim$ be a comparator in $\{<, \leq,=, \geq,>\}$. We write preds $0 f_{\mathrm{E}}^{\mathrm{B}}$ to mean the set of predicates (i.e., Boolean expressions) over Boolean variables B and arithmetic expressions E. A predicate $\pi$ in preds $0 f_{\mathrm{E}}^{\mathrm{B}}$ is either a Boolean value 
$\mathrm{b}$, a variable $\mathrm{v}^{b}$ in $\mathrm{B}$, a comparison of two expressions in $\mathrm{E}$ or a Boolean combination of predicates. It takes the following form:

$$
\pi \quad::=\quad \mathrm{b}\left|\mathrm{v}^{b}\right|(\mathrm{e} \sim \mathrm{e})|\neg \pi| \pi \wedge \pi \mid \pi \vee \pi \quad \mathrm{v}^{b} \in \mathrm{B}, \mathrm{e} \in \mathrm{E}
$$

We write $e(V)$ to emphasize that only variables in $\mathrm{V}$ are allowed to appear in e. We write varsOf(e) to mean all integer variables appearing in an expression e and varsOf $(\pi)$ to mean all variables appearing in $\pi$, namely both Boolean variables appearing in $\pi$ and all integer variables in vars0f(e) for each e appearing in $\pi$. We also write atoms $0 f(\pi)$ to mean all atoms appearing in $\pi$. An atom $\alpha$ is either a comparison (e $\sim$ e) of two arithmetic expressions or a Boolean. We write A to mean a set of atoms. For the set of integer variables $\mathrm{V}$, we write atoms $\mathrm{Of}(\mathrm{V})$ to mean the set of atoms over the variables V.

A mapping $\ltimes: X \rightarrow Y$ associates an element in $Y$ to each element in $X$. We write $x: X \rightarrow Y$ to mean a partial mapping from $X$ to $Y$. We write dom(x) and image( $x$ ) to respectively mean the domain and the image of $x$ and use $\epsilon_{X}: X \rightarrow Y$ for the mapping with an empty domain. We often write a partial mapping $\mathfrak{x}: X \rightarrow Y$ as the set $\{x \leftarrow \mathfrak{x}(x) \mid x \in \operatorname{dom}(\mathfrak{x})\}$ and write $\mathfrak{x} \cup \mathfrak{x}^{\prime}$ to mean the union of two mappings $x$ and $x^{\prime}$ with disjoint domains. We assume in the following an arithmetic or Boolean expression exp or any indexed version of it. We write $\exp [\vee]$ to mean the evaluation of expression exp for a mapping $\vee$. We evaluate as follows. First, an expression $\exp _{t m p}$ is deduced by syntactically and simultaneously replacing in exp each occurrence of a variable $\mathrm{v} \in \mathrm{V}$ by the corresponding $\vee(v)$. Then, if $\operatorname{varsOf}\left(\exp _{t m p}\right)=\varnothing$, $\exp [\mathrm{v}]$ is the constant obtained by evaluating $\exp _{t m p}$. Otherwise, $\exp [\mathrm{v}]$ is taken to be $\exp _{t m p}$ (see Example 6). Let $\vee: \mathrm{V} \rightarrow Y$ and $\nabla^{\prime}: \mathrm{V}^{\prime} \rightarrow Y^{\prime}$ be two mappings. If $\mathrm{V}$ and $\mathrm{v}^{\prime}$ are disjoint, we write $\exp \left[\vee, v^{\prime}\right]$ to mean the evaluation of expression exp with respect to $\vee \cup \vee^{\prime}$. Larger unions of mappings with pairwise disjoint domains are handled in a similar manner. We abuse notation and write $\vee\left[\vee^{\prime}\right]$ to mean $\left\{\mathrm{v} \leftarrow \mathrm{v}(\mathrm{v}) \mid \mathrm{v} \in \mathrm{V} \backslash \mathrm{V}^{\prime}\right\} \cup\left\{\mathrm{v} \leftarrow \mathrm{v}^{\prime}(\mathrm{v}) \mid \mathrm{v} \in \mathrm{V} \cap \mathrm{V}^{\prime}\right\}$.

Example 6 (Expressions). Let $\pi=\mathrm{v}^{b} \wedge\left(\mathrm{v}=\mathrm{v}^{\prime}+1\right)$. Then, $\operatorname{vars0f}(\pi)=$ $\left\{\mathrm{v}^{b}, \mathrm{v}, \mathrm{v}^{\prime}\right\}$. If we let $\mathrm{v}^{b}=\left\{\mathrm{v}^{b} \leftarrow\right.$ true $\}, \mathrm{v}_{1}=\left\{\mathrm{v} \leftarrow \mathrm{v}^{\prime}+5, \mathrm{v}^{\prime} \leftarrow 3\right\}$ and $\mathrm{v}_{2}=$ $\left\{\mathrm{v}^{\prime} \leftarrow-1\right\}$, then $\pi\left[\mathrm{v}^{b}, \nabla_{1}\right]=$ true $\wedge\left(\mathrm{v}^{\prime}+5=3+1\right)$ and $\pi\left[\mathrm{v}^{b}, \mathrm{v}_{1}\right]\left[\mathrm{v}_{2}\right]=$ true.

\subsection{Concurrent Programs and Counting Predicates}

To simplify the presentation, we assume a concurrent program (or program for short) to consist of a single non-recursive procedure manipulating integer variables. Arguments and return values are passed using shared variables. Programs where arbitrarily many processes run a finite number of procedures can be encoded by having the processes choose a procedure at the beginning. 


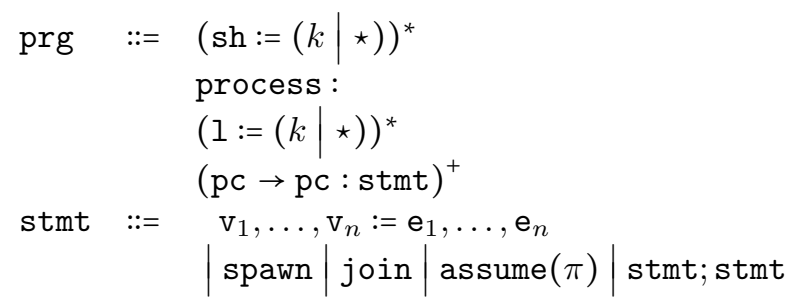

Figure 6.3: Syntax of concurrent programs: $\mathrm{sh}$ is a shared variable in $\mathrm{Sh}, 1$ is a local variable in $\mathrm{L}, \mathrm{v}_{1}, \ldots, \mathrm{v}_{n}$ are pairwise different variables in $\mathrm{Sh} \cup \mathrm{L}$, $\mathrm{e}_{1}, \ldots, \mathrm{e}_{n}$ are arithmetic expressions in $\operatorname{exprs} \mathrm{f}(\mathrm{Sh} \cup \mathrm{L})$ and $\pi$ is a predicate in predsOf $\operatorname{exprsOf}(\mathrm{Sh} \cup \mathrm{L}) \cdot$ * is a non-deterministic boolean value.

Syntax. The procedure of a program $\operatorname{Pr}=(\mathrm{Sh}, \mathrm{L}, \mathrm{Tr})$ is given in terms of a finite set $\operatorname{Tr}$ of transitions, each of the form ( $p c \rightarrow p c^{\prime}:$ stmt). Each transition involves two program locations $\mathrm{pc}$ and $\mathrm{pc}^{\prime}$ and a statement stmt. Transitions operate on two finite sets of integer variables, namely a set Sh of shared variables and a set $\mathrm{L}$ of local variables. We write PC to mean the set of all locations appearing in $\mathrm{Tr}$. We always distinguish two locations, namely an entry location $\mathrm{pc}_{0}$ and an exit location $\mathrm{pc}_{\mathrm{x}}$. Program syntax is described in Figure 6.3.

Semantics. Initially, a single process starts executing the procedure with both local and shared variables initialized as stated in their respective declarations. Executions might involve an arbitrary number of spawned processes. The execution of any process (whether initial or spawned with a spawn statement) starts at the entry location $\mathrm{pc}_{0}$ with the corresponding local variables initialized as stated in their respective declarations. Any process at an exit point $\mathrm{pc}_{\mathrm{x}}$ can be eliminated by a process executing a join statement. An assume $(\pi)$ statement blocks if the predicate $\pi$ over local and shared variables does not evaluate to true. Each transition is executed atomically by a single process without interruption from other processes.

A configuration is given in terms of a shared state and a group configuration. A shared state $\mathbb{S}: \mathrm{Sh} \rightarrow \mathbb{Z}$ is a mapping that associates an integer to each variable in $\mathbf{S h}$. We write $\mathbb{S}$ to mean the set of all shared states. An initial shared state is a mapping in $\mathbb{S}$ that respects shared variable declarations. We write $\mathbb{S}_{\text {init }}$ to mean the set of all initial shared states. A process state is a pair $(\mathrm{pc}, 0)$ where the location pc belongs to $\mathrm{PC}$ and the local state $0: \mathrm{L} \rightarrow \mathbb{Z}$ maps each local variable to an integer number. We also write $\mathbb{L}$ and $\mathbb{L}_{\text {init }}$ to respectively mean the sets of all local states and all initial local states. A group configuration is a multiset $m$ over process states. An initial group configuration maps all $(\mathrm{pc}, 0)$ to 0 except for a single $\left(\mathrm{pc}_{0}, 0\right)$, with $0 \in \mathbb{L}_{\text {init }}$, mapped to 1 . We write $M$ and $M_{\text {init }}$ to mean the sets of all group configurations and initial group 


$$
\begin{aligned}
& (s, 0, m) \underset{\operatorname{Pr}}{\stackrel{\text { stmt }}{\longrightarrow}}\left(s^{\prime}, \nabla^{\prime}, \mathrm{m}^{\prime}\right)
\end{aligned}
$$

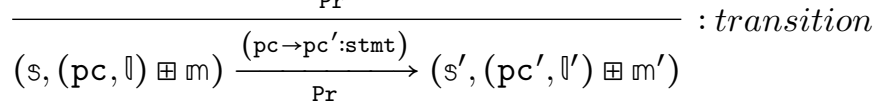

$$
\begin{aligned}
& (s, 0, \mathrm{~m}) \underset{\operatorname{Pr}}{\stackrel{\mathrm{stmt}}{\longrightarrow}}\left(s^{\prime}, \nabla^{\prime}, \mathrm{m}^{\prime}\right) \text { and }\left(\mathbb{s}^{\prime}, \nabla^{\prime}, \mathrm{m}^{\prime}\right) \underset{\operatorname{Pr}}{\stackrel{\mathrm{stmt}^{\prime}}{\longrightarrow}}\left(s^{\prime \prime}, 0^{\prime \prime}, \mathrm{m}^{\prime \prime}\right) \\
& (s, 0, \mathrm{~m}) \underset{\mathrm{Pr}}{\stackrel{\text { stmt; } \mathrm{stmt}^{\prime}}{\longrightarrow}}\left(\mathrm{s}^{\prime \prime}, \mathrm{0}^{\prime \prime}, \mathrm{m}^{\prime \prime}\right) \\
& \frac{\nabla=\left\{\mathrm{v}_{i} \leftarrow \mathrm{e}_{i}[\mathrm{~s}, 0] \mid i: 1 \leq i \leq n\right\}}{(\mathrm{s}, 0, \mathrm{~m}) \underset{\operatorname{Pr}}{\stackrel{v_{1}, \ldots, v_{n},:=\mathrm{e}_{1}, \ldots, \mathrm{e}_{n}}{\longrightarrow}}(\mathbb{s}[\mathrm{\vee}], 0[\mathrm{\vee}], \mathrm{m})} \text { : assign }
\end{aligned}
$$

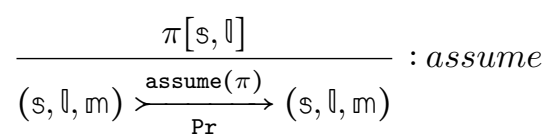

$$
\begin{aligned}
& \frac{\mathrm{m}^{\prime}=\left(\mathrm{pc}_{0}, \mathbb{0}_{\text {init }}\right) \boxplus \mathrm{m} \text { with } \nabla_{\text {init }} \in \mathbb{L}_{\text {init }}}{(\mathrm{s}, \mathbb{0}, \mathrm{m}) \underset{\mathrm{Pr}}{\stackrel{\text { spawn }}{\longrightarrow}}\left(\mathrm{s}, \mathbb{0}, \mathrm{m}^{\prime}\right)}: \text { spawn } \\
& \frac{\mathrm{m}=\left(\left(\mathrm{pc}_{\mathrm{x}}, \mathbb{D}^{\prime}\right) \boxplus \mathrm{m}^{\prime}\right)}{(\mathrm{s}, 0, \mathrm{~m}) \underset{\mathrm{Pr}}{\stackrel{\text { join }}{\longrightarrow}}\left(\mathrm{s}, 0, \mathrm{~m}^{\prime}\right)}: \text { join }
\end{aligned}
$$

Figure 6.4: Semantics of concurrent programs. Executions start from some $\left(\mathrm{pc}_{0}, \mathrm{~m}_{\text {init }}\right)$ with $\mathrm{m}_{\text {init }} \in M_{\text {init }}$. The operator $\boxplus$ is used to add a process state to $\mathrm{m}$.

configurations, respectively. Finally, a configuration is a pair $(s, \mathrm{~m})$ consisting of a shared state $s$ and a group configuration $m$. We write $(s, m) \underset{\operatorname{Pr}}{\stackrel{\operatorname{tr}}{\longrightarrow}}\left(s^{\prime}, \mathrm{m}^{\prime}\right)$ to mean that the transition $\operatorname{tr}$ of the form ( $\left.\mathrm{pc} \rightarrow \mathrm{pc^{ \prime }}: \mathrm{stmt}\right)$ applies atomically to configuration $(s, m)$ and changes it to $\left(s^{\prime}, \mathrm{m}^{\prime}\right)$. We introduce a relation $\underset{\mathrm{Pr}}{\stackrel{\text { stmt }}{\longrightarrow}}$ in order to describe the steps involved in the semantics of transitions (Figure 6.4). We write $(s, 0, m) \underset{\mathrm{Pr}}{\stackrel{\mathrm{stmt}}{\longrightarrow}}\left(s^{\prime}, 0^{\prime}, \mathrm{m}^{\prime}\right)$, where $s, s^{\prime}$ are shared states, $0,0^{\prime}$ are local states, and $\mathrm{m}, \mathrm{m}^{\prime}$ are multisets of group configurations, in order to mean

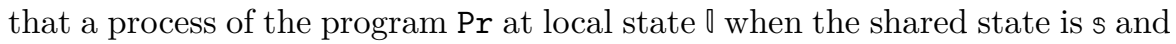
the other process states are captured by $m$, can execute the statement stmt and take the program to a configuration where the process has local state $\nabla^{\prime}$, the shared state is $s^{\prime}$ and the states of the other processes are captured by $\mathrm{m}^{\prime}$. For instance, a process can always execute a join if there is another process at location $\mathrm{pc}_{\mathrm{x}}$ (rule join). A process executing a multiple assignment atomically updates values of the shared and local variables according to the 
values taken by the expressions of the assignment before the execution (rule assign). A Pr-run $\rho$ starts with a configuration and is an alternating sequence of transitions and configurations $\left(s_{0}, \mathrm{~m}_{0}\right) ; \operatorname{tr}_{1} \ldots ; \operatorname{tr}_{n} ;\left(\mathbb{s}_{n}, \mathrm{~m}_{n}\right)$. The run is Pr-feasible if $\left(s_{i}, \mathrm{~m}_{i}\right) \underset{\operatorname{Pr}}{\stackrel{\operatorname{tr}_{i+1}}{\longrightarrow}}\left(s_{i+1}, \mathrm{~m}_{i+1}\right)$ for each $i: 0 \leq i<n$ and $s_{0}$ and $\mathrm{m}_{0}$ are initial, i.e., $\mathbb{s}_{0} \in \mathbb{S}_{\text {init }}$ and $\mathrm{m}_{0} \in \mathbb{M}_{\text {init }}$. Each configuration $\left(s_{i}, \mathrm{~m}_{i}\right)$ for $i: 0 \leq i \leq n$ is then said to be reachable.

Example 7 (Feasible run). Consider the concurrent program in Figure 6.1. An initial configuration is $\left(s_{0}, \mathbb{m}_{0}\right)$ where $s_{0}=$ $\{$ all $\leftarrow 1$, arrived $\leftarrow 0$, cross $\leftarrow 0$, read $\leftarrow 0\}$ and the initial multiset $m_{0}$ associates 1 to the unique process state with location $\mathrm{pc}_{0}$ and 0 to all other process states. A feasible run is then represented in the following.

\begin{tabular}{|c|c|c|c|c|}
\hline \multicolumn{4}{|c|}{ S } & \multirow{2}{*}{$\frac{\mathrm{m}}{\mathrm{pc}}$} \\
\hline all & arrived & cross & read & \\
\hline 1 & 0 & $\mathbf{0}$ & 0 & $\mathrm{pc}_{0}$ \\
\hline \multicolumn{5}{|c|}{$\Downarrow \mathrm{tr}_{0} \cdot \mathrm{pc}_{0} \rightarrow \mathrm{pc}_{0}:$ spawn } \\
\hline 1 & $\mathbf{0}$ & $\mathbf{0}$ & $\mathbf{0}$ & $\mathrm{pc}_{0}, \mathrm{pc}_{0}$ \\
\hline \multicolumn{5}{|c|}{$\Downarrow t r_{0} \cdot \mathrm{pc}_{0} \rightarrow \mathrm{pc}_{0}:$ spawn } \\
\hline 1 & $\mathbf{0}$ & $\mathbf{0}$ & $\mathbf{0}$ & 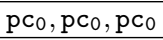 \\
\hline \multicolumn{5}{|c|}{$\Downarrow \operatorname{tr}_{1} \cdot \mathrm{pc}_{0} \rightarrow \mathrm{pc}_{1}: \operatorname{cross}=0 ; \mathrm{all}:=\mathrm{all}+1$} \\
\hline 2 & 0 & $\mathbf{0}$ & $\mathbf{0}$ & $\mathrm{pc}_{0}, \mathrm{pc}_{0}, \mathrm{pc}_{1}$ \\
\hline \multicolumn{5}{|c|}{$\Downarrow \operatorname{tr}_{2} \cdot \mathrm{pc}_{1} \rightarrow \mathrm{pc}_{2}:$ read $:=1$} \\
\hline 2 & $\mathbf{0}$ & $\mathbf{0}$ & 1 & $\mathrm{pc}_{0}, \mathrm{pc}_{0}, \mathrm{pc}_{2}$ \\
\hline \multicolumn{5}{|c|}{$\Downarrow \operatorname{tr}_{3} \cdot \mathrm{pc}_{2} \rightarrow \mathrm{pc}_{3}:$ read $:=0$} \\
\hline 2 & $\mathbf{0}$ & $\mathbf{0}$ & $\mathbf{0}$ & $\mathrm{pc}_{0}, \mathrm{pc}_{0}, \mathrm{pc_{3 }}$ \\
\hline
\end{tabular}

Counting Predicates. Recall that PC is the set of program locations. We make use of a set of Boolean variables $@ P C=\{@ p c \mid p c \in P C\}$. Intuitively, a process evaluates @pc to true only when it is at location pc. This way,

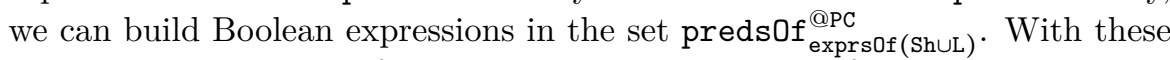
predicates we can state facts about both the location of a process, as well as its local variables and the values of the shared variables. For instance, at the fourth step of the run depicted in Example 7, there is one process for which $\left(@ \mathrm{pc}_{2} \wedge \mathrm{all} \geq 1\right)$ holds.

We associate a counting variable $(\pi)^{\#}$ to each predicate $\pi$ in preds $0 f_{\text {exprsOf }(\mathrm{Sh} \cup \mathrm{L})}^{\mathrm{QPC}}$. Intuitively, in a given program configuration, the variable $(\pi)^{\#}$ counts the number of processes for which the predicate $\pi$ holds. We

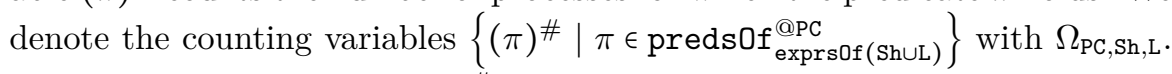
For example, $\left(@ \mathrm{pc}_{2} \wedge \mathrm{all} \geq 1\right)$ \# is a variable that counts the number of processes for which $\left(@ \mathrm{pc}_{2} \wedge \mathrm{all} \geq 1\right)$ holds. Such a counting variable is evaluated with respect to a shared state $s$ and a process state $(\mathrm{pc}, 0)$. We abuse notation and write $\mathrm{v}[\mathrm{s},(\mathrm{pc}, 0)]$ to mean that the variable $\mathrm{v}$ participating in a counting variable, is evaluated to $s(v)$ if $v \in S h$, to $\nabla(v)$ if $v \in L$, or to $\left(p c=p c^{\prime}\right)$ if $v$ is the Boolean variable @pc'. 
Any predicate in preds $0 f_{\text {exprsof }\left(\mathrm{Sh} \cup \Omega_{\mathrm{Pc}, \mathrm{Sh}, \mathrm{L}}\right)}$ is a counting predicate. We need a shared state $\$$ and a group configuration $m$ in order to evaluate a counting variable (Equation 6.1) or a counting predicate (Equation 6.2). We abuse notation and write $\omega[\mathrm{s}, \mathrm{m}]$ to mean the evaluation of the counting predicate $\omega$ with respect to a configuration $(s, m)$. The evaluation is performed as follows. Given a configuration $(s, \mathrm{~m})$, a shared variable $s h \in \mathrm{Sh}$ is evaluated as usual to $\operatorname{sh}[s, \mathrm{~m}]=\$(\mathrm{sh})$; whereas the counting variable $(\pi)^{\#}$ is evaluated to the number of processes satisfying $\pi$ in $(s, m)$.

$$
\begin{array}{r}
(\pi)^{\#}[\mathrm{~s}, \mathrm{~m}]=\sum_{\{(\mathrm{pc}, 0) \text { s.t. } \pi[\mathrm{s},(\mathrm{pc}, 0)]\}} \mathrm{m}((\mathrm{pc}, 0)) \\
\omega[\mathrm{s}, \mathrm{m}]=\omega\left[\mathrm{s},\left\{(\pi)^{\#} \leftarrow(\pi)^{\#}[\mathrm{~s}, \mathrm{~m}] \mid(\pi)^{\#} \in \operatorname{vars0f}(\omega)\right\}\right]
\end{array}
$$

Our counting predicates are quite expressive. For instance, we can capture assertion violations, deadlocks or rich program invariants with them (see Example 8). For any pc, we can define a counting predicate isEnabled(pc) that captures whether a process currently at location pc can fire some transition. For instance, in the running example of Figure 6.1 , isEnabled $\left(\mathrm{pc}_{0}\right)=$ true and isEnabled $\left(\mathrm{pc}_{4}\right)=($ arrived $=\mathrm{all})$. If there would have been only one transition from $\mathrm{pc}_{6}$ consisting of a join operation, then isEnabled $\left(\mathrm{pc}_{6}\right)=$ $\left(@ \mathrm{pc}_{\mathrm{x}} \geq 1\right)^{\#}$.

Example 8 (Counting predicates). The following counting predicates capture configurations of the program of Figure 6.1: $\omega_{1}$ captures configurations that violate the assertion, $\omega_{2}$ captures those in which a deadlock occurs, and $\omega_{3}$ captures an over-approximation of the reachable configurations (i.e., an invariant).

$$
\begin{aligned}
& \omega_{1}=\left(@ \mathrm{pc}_{5} \wedge(\operatorname{read}>0)\right)^{\#} \geq 1 \\
& \omega_{2}=\wedge_{\mathrm{pc} \in \mathrm{PC}}(@ \mathrm{pc} \wedge \text { isEnabled }(\mathrm{pc}))^{\#}=0 \\
& \omega_{3}=\left(\operatorname{all}=\sum_{i \geq 1}\left(@ \mathrm{pc}_{\mathrm{i}}\right)^{\#}\right) \wedge\left(\operatorname{arrived}=\sum_{i \geq 4}\left(@ \mathrm{pc}_{\mathrm{i}}\right)^{\#}\right)
\end{aligned}
$$

\subsection{Relating abstraction layers}

In the following, we formally describe the four steps involved in our predicated constrained monotonic abstraction approach (see Figure 6.2).

\subsubsection{Predicate abstraction}

Predicate abstraction [67] was introduced as a way of dealing with state explosion. In this approach, the state space of a program is approximated via the 


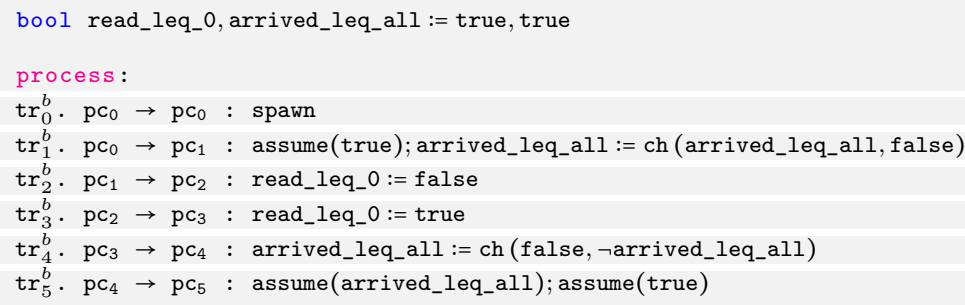

Figure 6.5: Predicate abstraction of the program in Figure 6.1 with respect to the predicates $\Pi=\{$ read_leq_0, arrived_leq_all $\}$. ch $\left(\pi, \pi^{\prime}\right)$ operator evaluates to true if $\pi$ evaluates to true, to false if $\pi^{\prime}$ evaluates to true, and non-deterministically to either true or false, otherwise.

values of a finite set of predicates over the program variables while maintaining the control structure of the program. The first algorithm for automatizing predicate abstraction of software programs was introduced in [14] and was a key enabling technology for applying finite-state model checkers to programs written in mainstream languages. The work in [51] extended predicate abstraction to shared-variable concurrent software. The authors have developed a symmetry-aware predicate abstraction strategy. The approach takes into account the replicated structure of $\mathrm{C}$ programs that consist of many threads executing the same procedure, and generates a Boolean program template whose multi-threaded execution soundly over-approximates the concurrent $\mathrm{C}$ program. State explosion during model checking parallel instantiations of this template can then be absorbed by exploiting symmetry. Predicate abstraction is usually combined with a CEGAR loop to add the required predicates and refine the abstraction.

Given a program $\operatorname{Pr}=(\mathrm{Sh}, \mathrm{L}, \mathrm{Tr})$ and a number of predicates $\Pi$ on the variables $\mathrm{Sh} \cup \mathrm{L}$, we leverage the technique in [51] in order to generate an abstraction in the form of a Boolean program abst $t_{\Pi}(\mathrm{Pr})=\left(\mathrm{Sh}^{b}, \mathrm{~L}^{b}, \operatorname{Tr}^{b}\right)$ where all shared and local variables are Boolean.

To achieve this, $\Pi$ is partitioned into three sets $\Pi_{s h r}, \Pi_{l o c}$ and $\Pi_{m i x}$. Predicates in $\Pi_{s h r}$ only mention variables in Sh and those in $\Pi_{l o c}$ only mention variables in $\mathrm{L}$. Predicates in $\Pi_{m i x}$ mention both shared and local variables of Pr. A bijection associates a predicate originOf $\left(\mathrm{v}^{b}\right)$ in $\Pi_{s h r}$ (respectively, $\Pi_{m i x} \cup \Pi_{l o c}$ ) to each Boolean variable $\mathrm{v}^{b}$ in $\mathrm{Sh}^{b}$ (respectively, $\mathrm{L}^{b}$ ). The function is a bijection as each predicate will be associated to only one Boolean variable (that tracks the value of that predicate) and vice versa.

Example 9 (Predicate abstraction). Consider the concurrent program in Figure 6.1. We implemented the predicate abstraction of [51] which results, for the predicates $\Pi=\{$ read_leq_0,arrived_leq_all $\}$, in the Boolean 
program of Figure 6.5. A bijection associates the predicates arrived $\leq$ all and read $\leq 0$ in $\Pi_{s h r}$ respectively, to the Boolean variables arrived_leq_all and read_leq_o in $\mathrm{Sh}^{b}$ of the Boolean program.

In addition, there are as many transitions in $\operatorname{Tr}$ as in $\operatorname{Tr}^{b}$. For each $\left(\mathrm{pc} \rightarrow \mathrm{pc} c^{\prime}:\right.$ stmt) in $\mathrm{Tr}$ there is a corresponding $\left(\mathrm{pc} \rightarrow \mathrm{pc}^{\prime}: \operatorname{abst}_{\Pi}(\operatorname{stmt})\right.$ ) with the same source and destination locations $\mathrm{pc}$ and $\mathrm{pc}^{\prime}$, but with an abstracted statement abst ${ }_{\Pi}$ (stmt) that may operate on the variables in $\mathrm{Sh}^{b} \cup \mathrm{L}^{b}$. Moreover, abstracted statements can mention the local variables of passive processes, i.e., processes other than the one executing the transition. For this, we make use of the variables $\mathrm{L}_{p}^{b}=\left\{\mathrm{I}_{p}^{b} \mid \mathrm{I}^{b}\right.$ in $\left.\mathrm{L}^{b}\right\}$ where each $\mathrm{I}_{p}^{b}$ denotes local variable $1^{b}$ of passive processes. We use passive variables to capture broadcasts where local variables of all passive processes need to be updated. Note that such passive variables and broadcast transitions do not exist in the original concurrent programs to be verified, but are introduced after predicate abstraction of those programs as presented in [51]. They are essential to capture the behavior of the processes that exist in the system other than the process that actually executes a transition (respectively, passive and active processes).

Example 10 (Broadcast transition). Consider a concurrent program with shared and local variables sh and 1 , the assignment transition $\operatorname{tr}_{0}:: \mathrm{pc}_{1} \rightarrow$ $\mathrm{pc}_{2}: \mathrm{sh}:=1$ and the mixed predicate $\mathrm{mx}::(\mathrm{sh}=1)$ to be used for the predicate abstraction. Recall from Section 6.5.1 that such predicates are called mixed predicates as they contain both local and shared variables. Similar to local predicates, each process will have its own copy of a mixed predicate. However, unlike local predicates, mixed predicates are updated only by broadcast transitions. Before the assignment in $\mathrm{tr}_{0}$ is executed, consider two passive processes $P_{1}$ and $P_{2}$ having mixed predicates $\mathrm{mx}_{p_{1}}::\left(\mathrm{sh}=1_{p_{1}}\right)$ evaluating to true and $\mathrm{mx}_{p_{2}}::\left(\mathrm{sh}=1_{p_{2}}\right)$ evaluating to false and an active process $P_{a}$ having $\mathrm{mx}_{p_{a}}::\left(\mathrm{sh}=\mathrm{I}_{a}\right)$ evaluating to true. The active process will execute $\operatorname{tr} r_{0}$, hence, $\mathrm{sh}=I_{a}$ will hold after the transition. At this point, $\mathrm{mx}_{p_{1}}$ will hold only if $1_{a}=1_{p_{1}}$. But, $\mathrm{mx}_{p_{2}}$ will not hold. In fact, after the transition, all passive processes will be notified to update their corresponding mixed predicate with respect to their own valuation and that of the active process. This corresponds to a broadcast (More details in Example 11).

Syntax and semantics of Boolean programs. We describe the syntax of Boolean programs in Figure 6.6. Variables $\operatorname{sh}^{b}$ and $1^{b}$ are some variables respectively, in $\mathrm{Sh}^{b}$ and $\mathrm{L}^{b}$. Variables $\mathrm{v}_{1}^{b}, \ldots, \mathrm{v}_{n}^{b}$ are pairwise different and belong to $\mathrm{Sh}^{b} \cup \mathrm{L}^{b} \cup \mathrm{L}_{p}^{b}$. Predicate $\pi$ is in preds $0 \mathrm{f}^{\mathrm{Sh}^{b} \cup \mathrm{L}^{b}}$ (i.e., a Boolean combination of the Boolean variables in $\left.\mathrm{Sh}^{b} \cup \mathrm{L}^{b}\right)$ and predicates $\pi_{i}, \pi_{i}^{\prime}$ are in predsOf $\mathrm{Sh}^{b} \cup \mathrm{L}^{b} \cup \mathrm{L}_{p}^{b}$. By construction, the predicate abstraction of concurrent programs [51] does not involve assignments of passive variables to non-passive ones. 


$$
\begin{aligned}
\text { prog }::= & \left(\operatorname{sh}^{b}:=(\text { true } \mid \text { false } \mid *)\right)^{*} \\
& \text { process : } \\
& \left(1^{b}:=(\text { true } \mid \text { false } \mid *)\right)^{*} \\
& (\operatorname{pc} \rightarrow \operatorname{pc}: \operatorname{stmt})^{+} \\
\text {stmt }::= & \mathrm{v}_{1}^{b}, \ldots, \mathrm{v}_{n}^{b}:=\operatorname{ch}\left(\pi_{1}, \pi_{1}^{\prime}\right), \ldots, \operatorname{ch}\left(\pi_{n}, \pi_{n}^{\prime}\right) \\
& \text { spawn } \mid \text { join }|\operatorname{assume}(\pi)| \operatorname{stmt} ; \text { stmt }
\end{aligned}
$$

Figure 6.6: Syntax of concurrent Boolean programs

We describe the semantics of Boolean programs in Figure 6.7. We add the superscript $b$ to mean the Boolean program variant. For instance, we use $\mathbb{S}^{b}, \mathbb{L}^{b}$ and $\mathbb{M}^{b}$ to respectively mean the sets of shared states, local states and group configurations of Boolean programs. The $\operatorname{ch}\left(\pi, \pi^{\prime}\right)$ operator evaluates to true if $\pi$ evaluates to true, to false if $\pi^{\prime}$ evaluates to true, and nondeterministically to either true or false, otherwise. Apart from the fact that all variables are now Boolean and that we make use of the ch operator, the main difference between Figure 6.7 and Figure 6.4 is the assign statement as it may involve passive variables in order to capture broadcasts.

Example 11 (ch operator). Consider once again Example 10. Using mx, the assignment transition $\mathrm{tr}_{0}$ will be abstracted to $\operatorname{tr}_{0}^{b}:: \mathrm{pc}_{1} \rightarrow \mathrm{pc}_{2}: \mathrm{mx}, \mathrm{mx}_{p}:=$ true, $\mathrm{ch}\left(\mathrm{mx}_{p} \wedge \mathrm{mx}, \mathrm{mx}_{p} \oplus \mathrm{mx}\right)$ ( $\oplus$ is exclusive-or). Based on the semantics of the ch operator, the variable $\mathrm{mx}_{p}$ will evaluate to true if both $\mathrm{mx}_{p}$ and $\mathrm{mx}$ held before the assignment, to $\mathrm{false}$ if $\mathrm{mx}_{p} \oplus \mathrm{mx}$ held before the assignment, and to a nondeterministic Boolean value otherwise.

Let $\quad \mathrm{v}_{1}^{b}, \ldots, \mathrm{v}_{n}^{b} \quad:=\operatorname{ch}\left(\pi_{1}, \pi_{1}^{\prime}\right), \ldots, \operatorname{ch}\left(\pi_{n}, \pi_{n}^{\prime}\right)$ be an assignment. When describing the semantics in Figure 6.7, we write $\left(\Phi^{b}, 0^{b}, 0_{p}^{b}\right) \stackrel{\mathrm{v}_{1}^{b}, \ldots, \mathrm{v}_{n}^{b}:=\operatorname{ch}\left(\pi_{1}, \pi_{1}^{\prime}\right), \ldots, \operatorname{ch}\left(\pi_{n}, \pi_{n}^{\prime}\right)}{\operatorname{abst}_{\Pi}(\operatorname{Pr})}\left(\Phi^{b^{\prime}}, 0^{b^{\prime}}, 0_{p}^{b^{\prime}}\right)$ in order to mean that a process other than the one performing the assignment (i.e., a passive process) with local state $\nabla_{p}^{b}$ can move to $0_{p}^{b^{\prime}}$ when the active process moves from $\nabla^{b}$ to $0^{b^{\prime}}$ and the shared state changes from $s^{b}$ to $s^{b^{\prime}}$. The new shared state and the new local state of the active process are obtained from their older versions according to Equations 6.3 and 6.4. The local state $0_{p}^{b^{\prime}}$ of a passive process is obtained as follows. First, we change the domain of $\nabla_{p}^{b}$ from $\mathrm{L}^{b}$ to $\mathrm{L}_{p}^{b}$ and obtain $0_{p, 1}^{b}$ as described in Equation 6.5. Then we apply the assignment to obtain $\pi_{p, 2}^{b}$ according to Equation 6.6. Finally, we obtain $\nabla_{p}^{b^{\prime}}$ by changing the domain of $\mathbb{0}_{p, 2}^{b}$ from $\mathbb{L}_{p}^{b}$ back to $\mathbb{L}^{b}$. Notice that at least one $\mathbb{0}_{p}^{b^{\prime}}$ exists for each $\nabla_{p}^{b}$. Intuitively, this step corresponds to updating when during a broadcast an active process changes local state together with possibly many passive processes. 


$$
\begin{aligned}
\mathbb{s}^{b^{\prime}} & =s^{b}\left[\left\{\mathrm{v}_{i}^{b} \leftarrow \operatorname{ch}\left(\pi_{i}, \pi_{i}^{\prime}\right)\left[\mathrm{s}^{b}, \nabla^{b}\right] \mid \mathrm{v}_{i}^{b} \in \mathrm{Sh}^{b} \wedge i: 1 \leq i \leq n\right\}\right] \\
\nabla^{b^{\prime}} & =\nabla^{b}\left[\left\{\mathrm{v}_{i}^{b} \leftarrow \operatorname{ch}\left(\pi_{i}, \pi_{i}^{\prime}\right)\left[\mathrm{s}^{b}, \nabla^{b}\right] \mid \mathrm{v}_{i}^{b} \in \mathrm{L}^{b} \wedge i: 1 \leq i \leq n\right\}\right] \\
\nabla_{p, 1}^{b} & =\left\{\mathrm{I}_{p}^{b} \leftarrow \nabla_{p}^{b}\left(\mathrm{I}^{b}\right) \mid \mathrm{I}^{b} \in \mathrm{L}^{b}\right\} \\
\nabla_{p, 2}^{b} & =\nabla_{p, 1}^{b}\left[\left\{\mathrm{v}_{i}^{b} \leftarrow \operatorname{ch}\left(\pi_{i}, \pi_{i}^{\prime}\right)\left[\mathrm{s}^{b}, \nabla^{b}, \nabla_{p, 1}^{b}\right] \mid \mathrm{v}_{i}^{b} \in \mathrm{L}_{p}^{b} \wedge i: 1 \leq i \leq n\right\}\right]
\end{aligned}
$$

Example 12 (Updating a passive local state). Consider once again Example 10, Example 11, the active local state $0^{b}=\{\mathrm{mx} \leftarrow$ false $\}$ and a passive local state $0_{p}^{b}=\{\mathrm{mx} \leftarrow$ true $\}$. The assignment in the Boolean transition $\operatorname{tr}_{0}^{b}$ involves both variables $\mathrm{mx}$ and $\mathrm{mx}_{p}$. We use $\nabla^{b}$ and $\nabla_{p}^{b}$ for evaluating $\mathrm{mx}$ and $\mathrm{mx}_{p}$, respectively; but the domain of the passive local state $\nabla_{p}^{b}$ is $\mathrm{L}^{b}$ based on the syntax of Boolean concurrent programs. We need to change its domain to $\mathrm{L}_{p}^{b}$ in order to be able to distinguish between passive and non-passive variables. By doing this, we obtain $\nabla_{p, 1}^{b}=\left\{\mathrm{mx}_{p} \leftarrow\right.$ true $\}$. Then, we update $\nabla_{p, 1}^{b}$ by the result of the assignment and will have $0_{p, 2}^{b}=\left\{\mathrm{mx}_{p} \leftarrow\right.$ false $\}$. Finally, we obtain $\mathbb{0}_{p}^{b^{\prime}}$ by changing the domain of $\mathbb{0}_{p, 2}^{b}$ from $\mathbb{L}_{p}^{b}$ back to $\mathbb{L}^{b}$ as $\mathbb{0}_{p}^{b^{\prime}}=\{\mathrm{mx} \leftarrow$ false $\}$.

An $\operatorname{abst}_{\Pi}(\operatorname{Pr})$-run $\left(s_{0}^{b}, \mathrm{~m}_{0}^{b}\right) ; \mathrm{tr}_{1}^{b} ; \ldots ; \mathrm{tr}_{n}^{b} ;\left(\mathrm{s}_{n}^{b}, \mathrm{~m}_{n}^{b}\right)$ is a sequence of alternating transitions and configurations starting with a configuration. This run is considered feasible if we have that $\left(\mathbb{s}_{i}^{b}, \mathrm{~m}_{i}^{b}\right) \underset{\operatorname{abst}_{\Pi}(\mathrm{Pr})}{\stackrel{\operatorname{tr}_{i+1}^{b}}{\longrightarrow}}\left(\mathbb{s}_{i+1}^{b}, \mathrm{~m}_{i+1}^{b}\right)$ for each $i: 0 \leq i<n$ and $\mathbb{s}_{0}^{b}, \mathrm{~m}_{0}^{b}$ are in $\mathbb{S}_{i n i t}^{b}$ and $\mathbb{M}_{i n i t}^{b}$, respectively. Configurations $\left(\Phi_{i}^{b}, \mathrm{~m}_{i}^{b}\right)$, for $i: 0 \leq i \leq n$, are then said to be reachable.

Evaluating (counting) predicates in $\operatorname{abst}_{\Pi}(\mathrm{Pr})$. Given a shared state $\Phi^{b}$, we write originOf $\left(\Phi^{b}\right)$ to mean the predicate $\Lambda_{\operatorname{sh}^{b} \in \operatorname{Sh}^{b}}\left(\Phi^{b}\left(\operatorname{sh}^{b}\right) \Leftrightarrow\right.$ originOf $\left.\left(\operatorname{sh}^{b}\right)\right)$. We write originOf $\left(\nabla^{b}\right)$ to mean $\Lambda_{1^{b} \in \mathrm{L}^{b}}\left(\nabla^{b}\left(1^{b}\right) \Leftrightarrow\right.$ originOf $\left.\left(1^{b}\right)\right)$. Observe that varsOf(originOf $\left.\left(s^{b}\right)\right) \subseteq$ Sh and the variables $\left.\operatorname{vars0f(originOf}\left(\nabla^{b}\right)\right) \subseteq \mathrm{Sh} \cup \mathrm{L}$. We abuse notation and write $\mathbb{s}^{b}[s]$ (respectively, $\left.0^{b}[s, 0]\right)$ to mean that originOf $\left(s^{b}\right)[s]$ (respectively, originOf $\left(\nabla^{b}\right)[s, 0]$ ) holds.

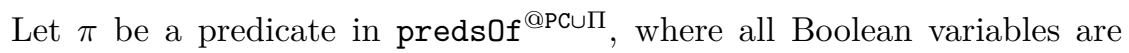
either predicates in $\Pi$ or of the form @pc for some pc $\in$ PC. We write $\pi\left[\mathrm{s}^{b},\left(\mathrm{pc}, \mathrm{0}^{b}\right)\right]$ to mean the Boolean value obtained by evaluating the result of replacing each Boolean variable @ $\mathrm{pc}^{\prime}$ with true if $\mathrm{pc}=\mathrm{pc} c^{\prime}$ and with false otherwise, and by replacing each $\pi^{\prime}$ in $\Pi$ with $\nabla^{b}\left(v^{b}\right)$ or $\mathbb{s}^{b}\left(v^{b}\right)$ where originOf $\left(\mathrm{v}^{b}\right)=\pi^{\prime}$.

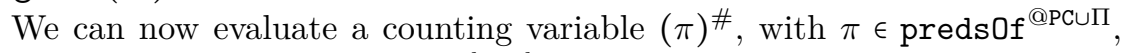
with respect to a configuration $\left(s^{b}, \mathrm{~m}^{b}\right)$. We do this by counting the number of process states $\left(\mathrm{pc}, 0^{b}\right)$ in $\mathrm{m}^{b}$ for which $\pi\left[\mathrm{s}^{b},\left(\mathrm{pc}, 0^{b}\right)\right]$ holds (see Equation $6.7)$. We can also replace each counting variable $(\pi)^{\#}$ in a counting predicate 


$$
\begin{aligned}
& \left(\Phi^{b}, \nabla^{b}, m^{b}\right) \underset{\operatorname{abst}_{\Pi}(\operatorname{Pr})}{\stackrel{\operatorname{stmt}}{\longrightarrow}}\left(s^{b^{\prime}}, \nabla^{b^{\prime}}, \mathrm{m}^{b^{\prime}}\right)
\end{aligned}
$$

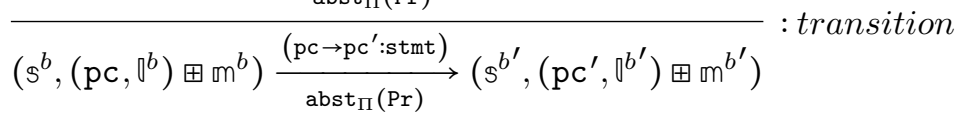

$$
\begin{aligned}
& \left(\Phi^{b}, \nabla^{b}, \mathrm{~m}^{b}\right) \underset{\operatorname{abst}_{\Pi}(\operatorname{Pr})}{\stackrel{\operatorname{stmt}}{\longrightarrow}}\left(\Phi^{b^{\prime}}, \nabla^{b^{\prime}}, \mathrm{m}^{b^{\prime}}\right) \quad\left(\Phi^{b^{\prime}}, \nabla^{b^{\prime}}, \mathrm{m}^{b^{\prime}}\right) \underset{\operatorname{abst}_{\Pi}(\mathrm{Pr})}{\stackrel{\mathrm{stmt}^{\prime}}{\longrightarrow}}\left(\Phi^{b^{\prime \prime}}, \nabla^{b^{\prime \prime}}, \mathrm{m}^{b^{\prime \prime}}\right) \\
& \left(\Phi^{b}, 0^{b}, \mathrm{~m}^{b}\right) \underset{\operatorname{abst}_{\Pi}(\mathrm{Pr})}{\stackrel{\text { stmt; } \text { stmt }^{\prime}}{\longrightarrow}}\left(\Phi^{b^{\prime \prime}}, 0^{b^{\prime \prime}}, \mathrm{m}^{b^{\prime \prime}}\right) \\
& \mathrm{m}=<\sigma_{1}, \sigma_{2}, \ldots, \sigma_{n}>\quad \mathrm{m}^{b}=<\sigma_{1}^{\prime}, \sigma_{2}^{\prime}, \ldots, \sigma_{n}^{\prime}> \\
& \text { a bijection } h:[1, n] \rightarrow[1, n] \text { s.t. } \\
& \forall i \in[1, n] . \sigma_{i}=\left(\mathrm{pc}_{\mathrm{p}}, 0_{p}^{b}\right) \quad \sigma_{h(i)}^{\prime}=\left(\mathrm{pc}_{\mathrm{p}}{ }^{\prime}, 0_{p}^{b^{\prime}}\right) \quad \mathrm{pc}_{\mathrm{p}}=\mathrm{pc}_{\mathrm{p}}{ }^{\prime} \\
& \left(\Phi^{b}, \nabla^{b}, 0_{p}^{b}\right) \stackrel{\mathrm{v}_{1}^{b}, \ldots, \mathrm{v}_{n}^{b}:=\operatorname{ch}\left(\pi_{1}, \pi_{1}^{\prime}\right), \ldots, \operatorname{ch}\left(\pi_{n}, \pi_{n}^{\prime}\right)}{\operatorname{abst}_{\Pi}(\operatorname{Pr})}\left(\Phi^{b^{\prime}}, \nabla^{b^{\prime}}, 0_{p}^{b^{\prime}}\right)
\end{aligned}
$$

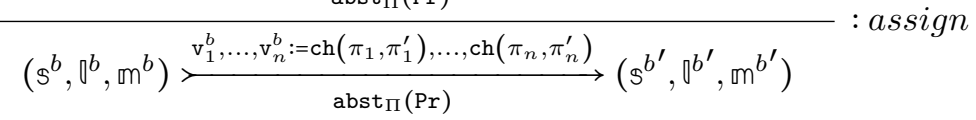

$$
\begin{aligned}
& \frac{\pi\left[s^{b}, \nabla^{b}\right]=\text { true }}{\left(s^{b}, \nabla^{b}, m^{b}\right) \underset{\operatorname{abst}_{\Pi}(\operatorname{Pr})}{\operatorname{assume}(\pi)}\left(s^{b}, \nabla^{b}, m^{b}\right)} \text { :assume } \\
& \frac{\mathrm{m}^{b^{\prime}}=\left(\left(\mathrm{pc}_{0}, \nabla_{\text {init }}^{b}\right) \boxplus \mathrm{m}^{b}\right) \quad \nabla_{\text {init }}^{b} \in \mathbb{L}_{\text {init }}^{b}}{\left(\mathrm{~s}^{b}, \mathbb{0}^{b}, \mathrm{~m}^{b}\right) \underset{\text { abst }_{\Pi}(\operatorname{Pr})}{\stackrel{\text { spawn }}{\longrightarrow}}\left(\mathrm{s}^{b}, \nabla^{b}, \mathrm{~m}^{b^{\prime}}\right)}: \text { spawn } \\
& \frac{\mathrm{m}^{b}=\left(\left(\mathrm{pc}_{\mathrm{x}}, 0^{b^{\prime}}\right) \boxplus \mathrm{m}^{b^{\prime}}\right)}{\left(\mathbb{s}^{b}, 0^{b}, \mathrm{~m}^{b}\right) \underset{\text { abst }_{\Pi}(\mathrm{Pr})}{\underset{\text { join }}{\longrightarrow}}\left(\mathbb{s}^{b}, 0^{b}, \mathrm{~m}^{b^{\prime}}\right)} \text { : join }
\end{aligned}
$$

Figure 6.7: Semantics of Boolean concurrent programs. Executions start from some $\mathrm{m}_{\text {init }}^{b}$ in $\mathbf{M}_{\text {init }}^{b}$. 
$\omega$ with its value in $\left(s^{b}, \mathrm{~m}^{b}\right)$, and each shared predicate in $\Pi$ with its value in $s^{b}$ (see Equation 6.8). Observe that the obtained expression might still involve shared variables as these can participate in comparisons with counting variables. Such comparisons do not correspond to any predicate in $\Pi$ and cannot therefore be mapped.

$$
\begin{array}{r}
(\pi)^{\#}\left[\mathrm{~s}^{b}, \mathrm{~m}^{b}\right]=\sum_{\left\{\left(\mathrm{pc}, 0^{b}\right) \mid\right.} \sum_{\left.\pi\left[\mathrm{s}^{b},\left(\mathrm{pc}, 0^{b}\right)\right]\right\}} \mathrm{m}^{b}\left(\left(\mathrm{pc}, 0^{b}\right)\right) \\
\omega\left[\mathrm{s}^{b}, \mathrm{~m}^{b}\right]=\omega\left[\left\{(\pi)^{\#} \leftarrow(\pi)^{\#}\left[\mathrm{~s}^{b}, \mathrm{~m}^{b}\right] \mid(\pi)^{\#} \in \operatorname{vars} 0 f(\omega)\right\}\right]
\end{array}
$$

Relation between $\operatorname{Pr}$ and abst $\mathrm{a}_{\Pi}(\mathrm{Pr})$. Given the group configurations $\mathrm{m}=<\sigma_{1}, \sigma_{2}, \ldots, \sigma_{n}>$ to $\mathrm{m}^{b}=<\sigma_{1}^{\prime}, \sigma_{2}^{\prime}, \ldots, \sigma_{n}^{\prime}>$, we let $\mathrm{m}^{b}[\mathrm{~s}, \mathrm{~m}]$ mean that there is a bijection $h$ from $[1, n]$ to $[1, n]$ such that for each $i$ in $[1, n]$, if $\sigma_{i}=(\mathrm{pc}, 0)$ then $\sigma_{h(i)}^{\prime}=\left(\mathrm{pc}, 0^{b}\right)$ and $0^{b}[\mathrm{~s}, 0]$. The concretization of an abst $t_{\Pi}(\mathrm{Pr})$ configuration $\left(\mathrm{s}^{b}, \mathrm{~m}^{b}\right)$ is $\gamma\left(\left(\mathrm{s}^{b}, \mathrm{~m}^{b}\right)\right)=$ $\left\{(s, m) \mid s^{b}[s] \wedge m^{b}[s, m]\right\}$. The abstraction of $(s, m)$ is the singleton $\alpha((s, m))=$ $\left\{\left(\mathrm{s}^{b}, \mathrm{~m}^{b}\right) \mid \mathrm{s}^{b}[\mathrm{~s}] \wedge \mathrm{m}^{b}[\mathrm{~s}, \mathrm{~m}]\right\}$. We initialize variables in abst $\mathrm{ab}_{\Pi}(\mathrm{Pr})$ such that for each pair $\left(s_{\text {init }}, \mathrm{m}_{\text {init }}\right)$ of $\mathrm{Pr}$, there are $\left(\mathrm{s}_{\text {init }}^{b}, \mathrm{~m}_{\text {init }}^{b}\right)$ that are initial in $\operatorname{abst}_{\Pi}(\mathrm{Pr})$ so that $\alpha\left(\left(\mathbb{s}_{\text {init }}, \mathrm{m}_{\text {init }}\right)\right)=\left\{\left(\mathbb{s}_{\text {init }}^{b}, \mathrm{~m}_{\text {init }}^{b}\right)\right\}$. The abstraction $\alpha(\rho)$ of a Pr-run $\rho=\left(\mathbb{s}_{0}, \mathrm{~m}_{0}\right) ; \operatorname{tr}_{1} ; \ldots ; \operatorname{tr}_{n} ;\left(\mathbb{s}_{n}, \mathrm{~m}_{n}\right)$ is the set of abst $\operatorname{abr}_{\Pi}(\operatorname{Pr})$ runs $\left\{\left(\mathbb{s}_{0}^{b}, \mathrm{~m}_{0}^{b}\right) ; \operatorname{tr}_{1}^{b} ; \ldots ; \operatorname{tr}_{n}^{b} ;\left(\mathbb{s}_{n}^{b}, \mathrm{~m}_{n}^{b}\right)\right\}$ where $\alpha\left(\left(s_{i}, \mathrm{~m}_{i}\right)\right)=\left\{\left(\mathbb{s}_{i}^{b}, \mathrm{~m}_{i}^{b}\right)\right\}$ and $\operatorname{tr}_{i}^{b}=$ $\operatorname{abst}_{\Pi}\left(t_{i}\right)$. Concretizations of abstract runs are defined in a straightforward manner.

Example 13. Consider the program in Figure 6.1, its corresponding abstraction with respect to the set of predicates $\Pi=$ \{read_leq_0,arrived_leq_all\} in Figure 6.5, and the feasible run in Example 7. The initial shared state $s_{0}$ defined as $\{$ all $\leftarrow 1$, arrived $\leftarrow$ 0 , cross $\leftarrow 0$, read $\leftarrow 0\}$ in the original program will be encoded as the Boolean shared state $s_{0}^{b}=\{$ read_leq_o $0 \leftarrow$ true, arrived_leq_all $\leftarrow$ true $\}$ in the Boolean program. We have that originof $\left(s_{0}^{b}\right)=(((\operatorname{read} \leq 0)=$ true $) \wedge(($ arrived $\leq$ all $)=$ true $)))$. Since there are no local variables in this example, we get that $\alpha\left(\left(s_{0}, \mathrm{~m}_{0}\right)\right)=\left\{\left(\Phi_{0}^{b}, \mathrm{~m}_{0}\right)\right\}$. Many other states have the same encoding, e.g. $\quad S_{1}=\{$ all $\leftarrow 10$, arrived $\leftarrow 2$, cross $\leftarrow 0$, read $\leftarrow 0\}$ which satisfies $s_{0}^{b}\left[\mathbb{s}_{1}\right]$, although it is not initial in $\mathrm{Pr}$. We get that $\gamma\left(\left(s_{0}^{b}, \mathrm{~m}_{0}\right)\right)=\left\{\left(\mathrm{s}_{0}, \mathrm{~m}_{0}\right),\left(\mathrm{s}_{1}, \mathrm{~m}_{0}\right), \ldots\right\}$.

Definition 11. Let the abstraction of the program $\operatorname{Pr}=(\mathrm{Sh}, \mathrm{L}, \mathrm{Tr})$ with respect to $\Pi$ be the Boolean program abst $t_{\Pi}(\mathrm{Pr})=\left(\mathrm{Sh}^{b}, \mathrm{~L}^{b}, \mathrm{Tr}^{b}\right)$. The abstraction is said to be effective and sound if abst $t_{\Pi}(\mathrm{Pr})$ can be effectively computed and the abstract run in the singleton $\alpha(\rho)$ of any feasible $\operatorname{Pr}$ run $\rho$ is abst $t_{\Pi}(\operatorname{Pr})$ feasible. 


$$
\begin{aligned}
& \frac{t=\left(q: o p: q^{\prime}\right) \mathbb{C} \underset{M}{\stackrel{o p}{\longrightarrow}} \mathbb{C}^{\prime}}{(q, \mathbb{C}) \underset{M}{\stackrel{t}{\longrightarrow}}\left(q^{\prime}, \mathbb{C}^{\prime}\right)} \text { transition }
\end{aligned}
$$

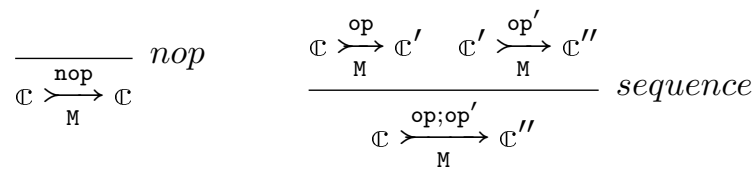

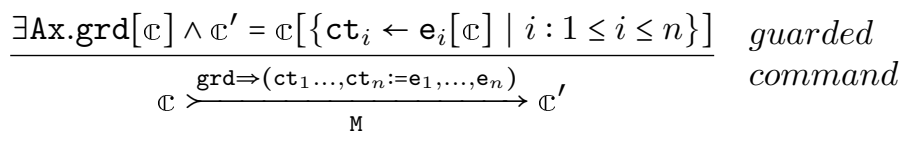

Figure 6.8: Semantics of an extended counter machine. A guard grd is a predicate in preds $0 f_{\text {exprsOf }(\mathcal{C} \cup A x)}$ in which $A x$ is a set of auxilliary variables used for a broadcast operation.

\subsubsection{Encoding into an Extended Counter Machine}

Assume a program $\operatorname{Pr}=(\mathrm{Sh}, \mathrm{L}, \mathrm{Tr})$, a set of predicates $\Pi_{0} \subseteq$ preds $0 f_{\text {exprsOf(ShuL) }}$ and two counting predicates, an invariant $\omega_{i n v}$ in the set preds $0 f_{\text {exprsOf }\left(\mathrm{Sh} \cup \Omega_{\mathrm{Pc}, \mathrm{Sh}, \mathrm{L}}\right)}$ and a target predicate $\omega_{\text {trgt }}$ in preds $0 f_{\operatorname{exprsOf}\left(\Omega_{\mathrm{Pc}, \mathrm{Sh}, \mathrm{L}}\right)}$. In the following, we write abst $t_{\Pi}(\operatorname{Pr})=\left(\mathrm{Sh}^{b}, \mathrm{~L}^{b}, \mathrm{Tr}^{b}\right)$ to mean the abstraction of $\operatorname{Pr}$ with respect to the predicates $\Pi$ defined as

$$
\Pi=\cup_{(\pi)} \# \in \operatorname{vars0f}\left(\omega_{\text {inv }}\right) \cup \operatorname{vars0f}\left(\omega_{\text {trgt }}\right) \text { atomsOf }(\pi) \cup \Pi_{0}
$$

Intuitively, this step results in the formulation of a state reachability problem of an extended counter machine enc $\left(\operatorname{abst}_{\Pi}(\mathrm{Pr})\right)$ that captures reachability of abstractions of $\omega_{\text {trgt }}$ configurations with abst $\log _{\Pi}(\mathrm{Pr})$ runs that take into account the invariant $\omega_{i n v}$. An extended counter machine $\mathrm{M}=$ $\left(\mathcal{Q}, \mathcal{C}, \mathrm{T}, \mathcal{Q}_{\text {init }}, \mathbb{C}_{\text {init }}, \mathrm{q}_{\text {trgt }}\right)$ is a tuple $\mathrm{M}=\left(\mathcal{Q}, \mathcal{C}, \mathrm{T}, \mathcal{Q}_{\text {init }}, \mathbb{C}_{\text {init }}, \mathrm{q}_{\text {trgt }}\right)$. A transition $t$ in $T$ is of the form $\left(q: o p: q^{\prime}\right)$ where $\operatorname{src}(t)=q$ is the source state, $\operatorname{dst}(\mathrm{t})=\mathrm{q}^{\prime}$ is the destination state, and the operation op is either the identity operation nop, a guarded command grd $\Rightarrow \mathrm{cmd}$, or a sequential composition of operations. We use a set $\mathrm{Ax}$ of auxiliary variables ranging over $\mathbb{N}$. These are meant to be existentially quantified when firing the transitions as explained in the guarded command rule in Figure 6.8 which explains the semantics of counter machines. A guard grd is a predicate in preds $0 f_{\text {exprsof }(\mathcal{C} \cup A x)}$ and a command $c m d$ is a multiple assignment $c t_{1}, \ldots, c t_{n}:=e_{1}, \ldots, e_{n}$ that involves $\mathrm{e}_{1}, \ldots, \mathrm{e}_{n}$ in exprsOf$(\mathcal{C} \cup \mathrm{Ax})$ and pairwise different $\mathrm{ct}_{1}, \ldots, \mathrm{ct}_{n}$ in $\mathcal{C}$.

Encoding. We describe in the following an extended counter machine enc $\left(\right.$ abst $\left._{\Pi}(\mathrm{Pr})\right)$ obtained as an encoding of the Boolean program abst $\mathrm{t}_{\Pi}(\mathrm{Pr})$. 


$$
\begin{aligned}
& \frac{t=\left(\mathrm{pc} \rightarrow \mathrm{pc}^{\prime}: \operatorname{stmt}_{\Pi}\right)\left[\left(\mathrm{s}^{b}, 0^{b}\right): \mathrm{op}:\left(\mathrm{s}^{b^{\prime}}, \mathrm{D}^{b^{\prime}}\right)\right]_{\mathrm{stmt}_{\Pi}}}{\left(\mathrm{q}_{\mathrm{s}^{b}}: \mathrm{ct}_{\left(\mathrm{pc}, b^{b}\right)} \geq 1 ; \mathrm{ct}_{\left(\mathrm{pc}, 0^{b}\right)}:=\mathrm{ct}_{\left(\mathrm{pc}, 0^{b}\right)}-1 ; \mathrm{op} ; \mathrm{ct}_{\left(\mathrm{pc}^{\prime}, b^{\prime}\right)}:=\mathrm{ct}_{\left(\mathrm{pc}^{\prime}, b^{\prime}\right)}+1: \mathrm{q}_{\mathrm{s}^{\prime}}\right) \in \mathrm{T}_{t}}: \text { transition }
\end{aligned}
$$

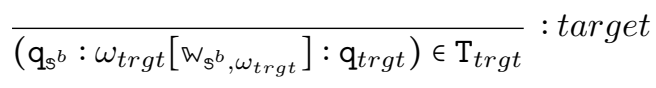

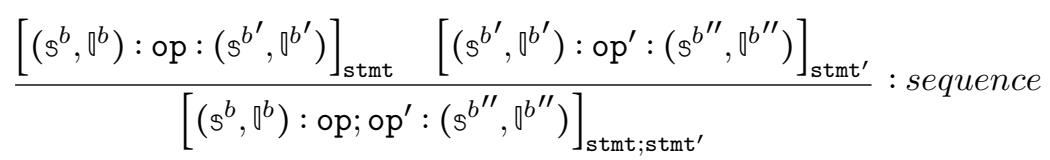

$$
\begin{aligned}
& \frac{\pi\left[\varsigma^{b}, \nabla^{b}\right]}{\left[\left(\varsigma^{b}, 0^{b}\right): \text { nop }:\left(s^{b}, 0^{b}\right)\right]_{\pi}}: \text { assume } \\
& \overline{\left[\left(\mathrm{s}^{b}, \nabla^{b}\right): \mathrm{ct}_{\left(\mathrm{pc}_{0}, 0^{b}{ }_{\text {init }}\right)}:=\mathrm{ct}_{\left(\mathrm{pc}_{0}, 0^{b}{ }_{\text {init }}\right)}+1:\left(\mathrm{s}^{b}, \mathbb{D}^{b}\right)\right]_{\text {spawn }}}: \text { spawn } \\
& \overline{\left[\left(\Phi^{b}, 0^{b}\right): \mathrm{ct}_{\left(\mathrm{pc}_{\mathrm{x}}, b^{\prime}\right)} \geq 1 ; \mathrm{ct}_{\left(\mathrm{pc}_{\mathrm{x}}, 0^{b^{\prime}}\right)}:=\mathrm{ct}_{\left(\mathrm{pc}_{\mathrm{x}}, b^{b^{\prime}}\right)}-1:\left(\mathbb{s}^{b}, 0^{b}\right)\right]_{\text {join }}}: \text { join } \\
& \operatorname{tf}=\left\{\left(\nabla_{p}^{b}, b_{p}^{b^{\prime}}\right) \mid\left(\Phi^{b}, \nabla^{b}, \nabla_{p}^{b}\right) \frac{\mathrm{v}_{1}^{b}, \ldots, \mathrm{v}_{n}^{b}:=\operatorname{ch}\left(\pi_{1}, \pi_{1}^{\prime}\right), \ldots, \operatorname{ch}\left(\pi_{n}, \pi_{n}^{\prime}\right)}{\operatorname{abst}_{\Pi}(\operatorname{Pr})}\left(\Phi^{b^{\prime}}, \nabla^{b^{\prime}}, \nabla_{p}^{b^{\prime}}\right)\right\} \\
& \text { tf } \neq \varnothing
\end{aligned}
$$

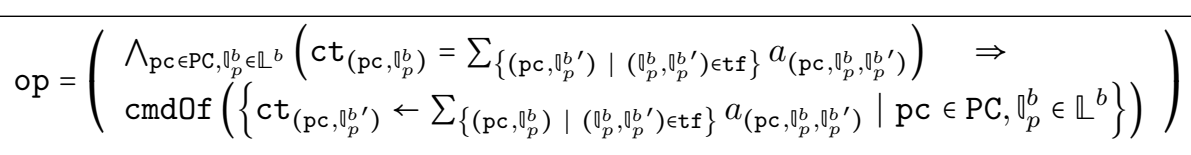

$$
\begin{aligned}
& {\left[\left(\Phi^{b}, \nabla^{b}\right): \text { op }:\left(\Phi^{b^{\prime}}, \nabla^{b^{\prime}}\right)\right]_{\text {assign }}}
\end{aligned}
$$

Figure 6.9: Encoding of the transitions of a Boolean program $\left(\mathrm{Sh}^{b}, \mathrm{~L}^{b}, \mathrm{Tr}^{b}\right)$, given a counting target $\omega_{\text {trgt }}$, to the transitions $T=\cup_{\operatorname{tr} \in \operatorname{Tr}^{b}} T_{\operatorname{tr}} \cup$ $\mathrm{T}_{\text {trgt }}$ of an extended counter machine. In rule assign, we write $\operatorname{cmdOf}\left(\left\{\mathrm{ct}_{\left(\mathrm{pc}, b^{b^{\prime}}\right)} \leftarrow \mathrm{e}_{\left(\mathrm{pc}, b^{b^{\prime}}\right)} \mid \mathrm{pc} \in \mathrm{PC}, 0^{b^{\prime}} \in \mathbb{L}^{b}\right\}\right)$ to mean the multiple assignment that simultaneously assigns each $e_{\left(p c, 0^{b}\right)}$ to $c t_{\left(p c, 0^{b}\right)}$. A transition $\mathbb{C}_{1} \underset{\text { abst }_{\Pi}(\operatorname{Pr})}{\stackrel{\operatorname{grd} \Rightarrow \mathrm{cmd}}{\longrightarrow}} \mathbb{C}_{2}$ ensures that there is a mapping $\mathbb{a}: \mathrm{Ax} \rightarrow \mathbb{N}$ from the set of auxiliary variables Ax to the set of natural numbers such that for any $\mathrm{ct}_{\left(\mathrm{pc},,_{p}^{b}\right)}$ we have $\mathbb{C}_{1}\left(\mathrm{ct}_{\left(\mathrm{pc},,_{p}^{b}\right)}\right)=\sum_{\left(0_{p}^{b}, b_{p}^{\prime}\right) \in \operatorname{tf}} \mathbb{a}\left(a_{\left(\mathrm{pc}, b_{p}^{b}, b_{p}^{\prime}\right)}\right)$ and for any $\mathrm{ct}\left(\mathrm{pc}, b_{p}^{\prime \prime}\right)$ we have $\mathbb{C}_{2}\left(\mathrm{ct}_{\left(\mathrm{pc}, b_{p}^{b^{\prime}}\right)}\right)=\sum_{\left(\nabla_{p}^{b}, b_{p}^{\prime}{ }^{\prime}\right) \in \mathrm{tf}} \mathbb{a}\left(a_{\left(\mathrm{pc}, 0_{p}^{b},,_{p}^{b \prime}\right)}\right)$. 
Recall that $\operatorname{abst}_{\Pi}(\mathrm{Pr})$ results from the predicate abstraction of the concurrent program $\mathrm{Pr}$ with respect to some initial predicates $\Pi_{0}$ as well as all predicates in atoms $0 f(\pi)$ for every counting variable $(\pi)^{\#}$ in $\operatorname{vars0f}\left(\omega_{i n v}\right)$ and $\operatorname{vars0f}\left(\omega_{\text {trgt }}\right)$. The machine enc $\left(\operatorname{abst}_{\Pi}(\operatorname{Pr})\right)$ is a tuple $\left(\mathcal{Q}, \mathcal{C}, \mathrm{T}, \mathcal{Q}_{\text {init }}, \mathbb{C}_{\text {init }}, \mathrm{q}_{\text {trgt }}\right)$. A state in $\mathcal{Q}$ is either the target state $\mathrm{q}_{\text {trgt }}$ or is associated to a shared configuration $\Phi^{b}$ of $\operatorname{abst}_{\Pi}(\mathrm{Pr})$. We write $\mathrm{q}_{\mathrm{s}^{b}}$ to make the association explicit. There is a bijection that associates a process configuration $\left(\mathrm{pc}, 0^{b}\right)$ to each counter $\mathrm{ct}_{\left(\mathrm{pc}, 0^{b}\right)}$ in $\mathcal{C}$. The assign rule makes use of auxiliary variables. In case of broadcasts, this rule associates an auxiliary variable $a_{\left(\mathrm{pc}, 0_{p}^{b}, 0_{p}^{b}\right)}$ to each possible move from process configuration ( $\left.\mathrm{pc}, 0_{p}^{b}\right)$ to $\left(\mathrm{pc},{ }_{p}^{b^{\prime}}\right)$.

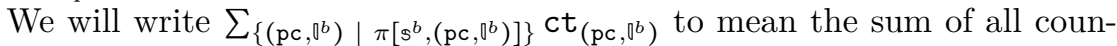
ters $\mathrm{ct}_{\left(\mathrm{pc}, 0^{b}\right)}$ in $\mathcal{C}$ for which $\pi\left[\mathrm{s}^{b},\left(\mathrm{pc}, 0^{b}\right)\right]$ evaluates to true. We can define the mapping $\mathbb{W}_{\mathbb{S}^{b}, \omega}$ that maps each counting variable appearing in a counting predicate $\omega$ to a corresponding sum of counters under $s^{b}$. More formally, $\mathbb{W}_{s^{b}, \omega}\left((\pi)^{\#}\right)=\sum_{\left\{\left(\mathrm{pc}, 0^{b}\right) \mid \pi\left[\mathrm{s}^{b},\left(\mathrm{pc}, 0^{b}\right)\right]\right\}} \mathrm{ct}_{\left(\mathrm{pc}, 0^{b}\right)}$ for each counting predicate $(\pi)^{\#}$ in $\operatorname{vars} 0 f(\omega)$. As a result, $\omega_{\text {trgt }}\left[\mathbb{W}_{\mathbb{S}^{b}, \omega}\right]$ is the predicate obtained from $\omega_{\text {trgt }}$ after replacing all counting variables appearing in it by the corresponding sums of counters. Observe that if the predicate $\omega$ is in preds $0 f_{\operatorname{exprs0f}\left(\Omega_{\mathrm{Pc}, \mathrm{Sh}, \mathrm{L}}\right)}$ as it is the case for $\omega_{\text {trgt }}$, then $\omega\left[\mathbb{W}_{\mathbb{s}^{b}, \omega}\right]$ does not mention any shared variables in Sh. The target predicate $\omega_{\text {trgt }}$ does not mention any shared variables, because it is to be evaluated in a configuration of the counter machine where no concrete value for shared variables is available. The predicate $\omega_{\operatorname{trgt}}\left[\mathrm{W}_{\mathrm{s}^{b}, \omega}\right]$ in preds $0 f_{\text {exprsOf }(\mathcal{C})}$ is used in the target rule of the encoding in Figure 6.9.

The set of transitions $T$ is exactly the set $\cup_{t r \in \operatorname{Tr}} b T_{t r} \cup T_{\text {trgt }}$ as described in Figure 6.9. We abuse notation and associate to each statement stmt appearing in abst $t_{\Pi}(\operatorname{Pr})$ the set enc (stmt) of tuples $\left[\left(\Phi^{b}, 0^{b}\right): \text { op }:\left(\Phi^{b^{\prime}}, 0^{b^{\prime}}\right)\right]_{\text {stmt }}$ generated in Figure 6.9. Given a group configuration $\mathrm{m}^{b}$, we write $\mathbb{C}_{\mathrm{m}^{b}}$ to mean the mapping associating $\mathrm{m}^{b}\left(\left(\mathrm{pc}, 0^{b}\right)\right)$ to each counter $\mathrm{ct}_{\left(\mathrm{pc}, 0^{b}\right)}$ in $\mathcal{C}$. We let $\mathcal{Q}_{\text {init }}$ be the set $\left\{\mathrm{q}_{s_{0}^{b}} \mid \mathbb{s}_{0}^{b} \in \mathbb{S}_{\text {init }}^{b}\right.$ of $\left.\operatorname{abst}_{\Pi}(\operatorname{Pr})\right\}$, and $\mathbb{C}_{\text {init }}$ be the mapping $\left\{\mathbb{C}_{\mathrm{m}^{b}} \mid \mathrm{m}^{b}\left(\left(\mathrm{pc}_{0}, \nabla^{b}\right)\right)=1\right.$ for a single $\nabla^{b} \in \mathbb{L}_{\text {init }}^{b}$ in abst $\mathrm{a}_{\Pi}(\mathrm{Pr})$ and 0 otherwise $\}$. We associate a program configuration $\left(\mathrm{s}^{b}, \mathrm{~m}^{b}\right)$ to each machine configuration $\left(\mathrm{q}_{\mathrm{s}^{b}}, \mathbb{C}_{\mathrm{m}^{b}}\right)$. The machine enc $\left(\operatorname{abst}_{\Pi}(\mathrm{Pr})\right)$ encodes abst $_{\Pi}(\mathrm{Pr})$ as specified in Lemma 26.

We state in Lemma 26 that the reachability problem of the obtained counter machine is equivalent to the reachability in $\operatorname{abst}_{\Pi}(\mathrm{Pr})$ of Boolean configurations satisfying $\omega_{\text {trgt }}$. For this, we make use of Lemma 24 and Lemma 25. Intuitively, these relate executions of the Boolean abstraction to the ones of the counter machine encoding. 
Lemma 24 (translation). For any stmt appearing in the program abst $t_{\Pi}(\mathrm{Pr}),\left(\mathbb{s}^{b}, 0^{b}, \mathrm{~m}^{b}\right) \underset{\text { abst }(\mathrm{Pr})}{\stackrel{\mathrm{stmt}}{\longrightarrow}}\left(\mathbb{s}^{b^{\prime}}, \nabla^{b^{\prime}}, \mathrm{m}^{b^{\prime}}\right)$ iff $\mathbb{C}_{\mathrm{m}^{b}} \underset{\text { enc(abst }(\mathrm{Pr}))}{\stackrel{\mathrm{op}}{\longrightarrow}} \mathbb{C}_{\mathrm{m}^{b^{\prime}}}$ for $a\left[\left(\Phi^{b}, \nabla^{b}\right): \text { op }:\left(\Phi^{b^{\prime}}, \nabla^{b^{\prime}}\right)\right]_{\text {stmt }}$ in enc (stmt).

Proof. By induction on the number of atomic statements in stmt.

Lemma 25 (translation and abstraction). Any configuration $\left(\mathrm{s}^{b}, \mathrm{~m}^{b}\right)$ is reachable in abst $t_{\Pi}(\mathrm{Pr})$ iff $\left(\mathrm{q}_{\mathrm{s}^{b}}, \mathbb{C}_{\mathrm{m}^{b}}\right)$ is reachable in enc $\left(\right.$ abst $\left._{\Pi}(\mathrm{Pr})\right)$.

Proof. We show that $\left(s^{b}, \mathrm{~m}^{b}\right)$ is reachable via a run of length $n$ in $\operatorname{abst}_{\Pi}(\operatorname{Pr})$ iff $\left(\mathrm{q}_{\mathrm{s}^{b}}, \mathbb{C}_{\mathrm{m}^{b}}\right)$ is reachable via a run of the same length in enc $\left(\mathrm{abst}_{\Pi}(\mathrm{Pr})\right)$. We proceed by induction on the number of abst $\mathrm{ar}_{\Pi}(\mathrm{Pr})$ transitions appearing in the runs. By construction, $s_{0}^{b}$ and $m_{0}^{b}$ are initial iff $\mathrm{q}_{s_{0}^{b}}$ and $\mathbb{C}_{\mathrm{m}_{0}^{b}}$ are also initial. Let $\left(\mathrm{s}^{b}, \mathrm{~m}^{b}\right)$ be a reachable abst $t_{\Pi}(\mathrm{Pr})$ configuration and $\left(\mathrm{q}_{\mathrm{s}^{b}}, \mathbb{C}_{\mathrm{m}^{b}}\right)$ be the corresponding enc $\left(\right.$ abst $_{\Pi}(\mathrm{Pr})$ ) configuration. We will not consider runs in enc $\left(\mathrm{abst}_{\Pi}(\mathrm{Pr})\right)$ that involve $\mathrm{T}_{\text {trgt }}$ transitions as these lead to error states and not to configura-

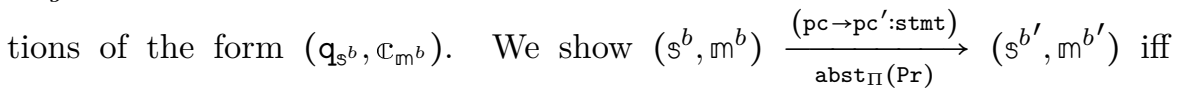
$\left(\mathrm{q}_{\mathrm{s}^{b}}, \mathbb{C}_{\mathrm{m}^{b}}\right) \frac{\left(\mathrm{q}_{\mathrm{s}} b: \mathrm{ct}_{\left(\mathrm{pc}, 0^{b}\right)} \geq 1 ; \mathrm{ct}_{\left(\mathrm{pc}, 0^{b}\right)}:=\mathrm{ct}_{\left(\mathrm{pc}, 0^{b}\right)}-1 ; o p ; \mathrm{ct}_{\left(\mathrm{pc}^{\prime}, b^{b^{\prime}}\right)}:=\mathrm{ct}_{\left(\mathrm{pc}^{\prime}, b^{\prime}\right)}+1: \mathrm{q}_{\mathrm{s}} b^{\prime}\right)}{\operatorname{enc}\left(\operatorname{abst}_{\Pi}(\mathrm{Pr})\right)}\left(\mathrm{q}_{\mathrm{s}^{b^{\prime}}}, \mathbb{C}_{\mathrm{m}^{b^{\prime}}}\right)$ for some $\left[\left(\Phi^{b}, 0^{b}\right): \text { op }:\left(\Phi^{b^{\prime}}, 0^{b^{\prime}}\right)\right]_{\text {stmt }}$ in enc (stmt). Semantics of Boolean programs in Figure 6.7 ensure that $\left(\Phi^{b}, \mathrm{~m}^{b}\right) \underset{\operatorname{abst}_{\Pi}(\mathrm{Pr})}{\stackrel{\left(\mathrm{pc} \rightarrow \mathrm{pc}^{\prime}: \mathrm{stmt}\right)}{\longrightarrow}}\left(\mathbb{s}^{b^{\prime}}, \mathrm{m}^{b^{\prime}}\right)$ iff $\mathrm{m}^{b}=$ $\left(\mathrm{pc}, \nabla^{b}\right) \oplus \mathrm{m}_{1}^{b}$ and $\mathrm{m}^{b^{\prime}}=\left(\mathrm{pc}^{\prime}, \nabla^{b^{\prime}}\right) \oplus \mathrm{m}_{1}^{b^{\prime}}$ and $\left(\mathbb{s}^{b}, \nabla^{b}, \mathrm{~m}_{1}^{b}\right) \underset{\text { abst }_{\Pi}(\operatorname{Pr})}{\stackrel{\operatorname{stmt}}{\longrightarrow}}\left(\mathbb{s}^{b^{\prime}}, \nabla^{b^{\prime}}, \mathrm{m}_{1}^{b^{\prime}}\right)$. Lemma 24 ensures that this is equivalent to $\mathbb{C}_{\mathrm{m}_{1}^{b}} \underset{\text { enc(abst }(\operatorname{Pr}))}{\stackrel{\mathrm{op}}{\longrightarrow}} \mathbb{C}_{\mathrm{m}_{1}^{b^{\prime}}}$ for some $\left[\left(s^{b}, 0^{b}\right): \text { op }:\left(s^{b^{\prime}}, 0^{b^{\prime}}\right)\right]_{\text {stmt }}$ in enc (stmt). Observe that $\mathbb{C}_{\mathrm{m}_{1}^{b}} \underset{\text { enc }\left(\operatorname{abst}_{\Pi}(\operatorname{Pr})\right)}{\stackrel{\text { op }}{\longrightarrow}} \mathbb{C}_{\mathrm{m}_{1}^{b^{\prime}}}$

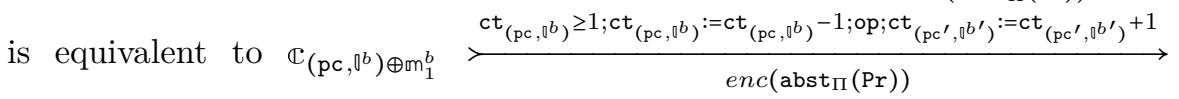
$\mathbb{C}_{\left(\mathrm{pc}^{\prime}, 0^{b^{\prime}}\right) \oplus \mathrm{m}_{1}^{b^{\prime}}}$

Lemma 26 (translation reachability). Target $\mathrm{q}_{\text {trgt }}$ is enc $\left(\right.$ abst $\left._{\Pi}(\mathrm{Pr})\right)$ reachable iff a configuration $\left(\mathrm{s}^{b}, \mathrm{~m}^{b}\right)$ is reachable in abst $t_{\Pi}(\mathrm{Pr})$ such that $\omega_{\text {trgt }}\left[\mathrm{s}^{b}, \mathrm{~m}^{b}\right]$ holds.

Proof. Lemma 25 ensures that $\left(\mathbb{s}^{b}, \mathrm{~m}^{b}\right)$ is $\operatorname{abst}_{\Pi}(\operatorname{Pr})$ reachable iff $\left(\mathrm{q}_{s^{b}}, \mathbb{C}_{\mathrm{m}^{b}}\right)$ is enc $\left(\operatorname{abst}_{\Pi}(\mathrm{Pr})\right)$ reachable. We conclude by observing that $\omega_{\text {trgt }}\left[\mathrm{s}^{b}, \mathrm{~m}^{b}\right]$ holds iff the evaluation of the target predicate in the counter machine holds, i.e. $\omega_{\text {trgt }}\left[\mathrm{q}_{\mathrm{s}^{b}}, \mathbb{C}_{\mathrm{m}^{b}}\right]$ holds. 


\subsubsection{Encoding precision}

We argue in the following that the obtained counter machine results in a monotonic transition system for which the reachability problem is decidable. In fact, predicate abstraction forces monotonicity. For example, in Figure 6.1, transitions $\operatorname{tr}_{4}$ and $\operatorname{tr}_{5}$ correspond to a barrier which is non-monotonic. However, the abstracted Boolean program in Figure 6.5 that corresponds to it consists of only monotonic transitions. This happens because the relation between the number of processes in different program locations and the program variables is lost. This corresponds to a loss of precision that makes it impossible to establish correctness of programs such as the one depicted in Figure 6.1. We explain how we retrieve some of that precision by strengthening the abstraction.

Consider the Boolean program abst $t_{\Pi}(\mathrm{Pr})$ obtained after predicate abstraction. If a configuration $\left(\mathrm{s}^{b^{\prime}}, \mathrm{m}^{b^{\prime}}\right)$ is obtained from $\left(\mathrm{s}^{b}, \mathrm{~m}^{b}\right)$ using some transition, then the same transition can obtain a larger configuration (i.e., has the same shared state $s^{b^{\prime}}$ and more processes at the same process states in $\left.\mathrm{m}^{b^{\prime}}\right)$ than $\left(\mathrm{s}^{b^{\prime}}, \mathrm{m}^{b^{\prime}}\right)$ from any configuration larger than $\left(\mathrm{s}^{b}, \mathrm{~m}^{b}\right)$. Lemma 27 shows that indeed, all transitions in Figure 6.9 (except for the rule target) are monotonic with respect to the ordering $\leq$ defined by $(\mathrm{q}, \mathbb{C}) \leq\left(\mathrm{q}^{\prime}, \mathbb{C}^{\prime}\right)$ iff $\mathrm{q}=\mathrm{q}^{\prime}$ and $\mathbb{C} \unlhd \mathbb{C}^{\prime}$ with respect to the component-wise ordering, i.e. $\mathbb{C} \unlhd \mathbb{C}^{\prime}$ iff $\mathbb{C}(\mathrm{ct}) \leq \mathbb{C}^{\prime}(\mathrm{ct})$ for each $\mathrm{ct} \in \mathcal{C}$.

Lemma 27 (monotonicity). Transitions ( $\left.\mathrm{q}: \mathrm{op}: \mathrm{q}^{\prime}\right)$ generated by all rules in Figure 6.9, except for the target rule are monotonic with respect to $\leq$.

Proof. Let op be some operation appearing in a generated transition (q : op : $\left.\mathrm{q}^{\prime}\right)$ of enc $\left(\operatorname{abst}_{\Pi}(\mathrm{Pr})\right)$. We say that an operation op is monotonic with respect to $\leq$ if for each $\mathbb{C}_{1}, \mathbb{C}_{2}, \mathbb{C}_{3}$ such that $\mathbb{C}_{1} \underset{\text { enc }\left(\mathrm{abst}_{\Pi}(\mathrm{Pr})\right)}{\stackrel{\text { op }}{\longrightarrow}} \mathbb{C}_{2}$ and $\mathbb{C}_{1} \leq \mathbb{C}_{3}$ there exists a $\mathbb{C}_{4}$ such that $\mathbb{C}_{3} \underset{\text { enc }\left(\text { abst }_{\Pi}(\mathrm{Pr})\right)}{\text { op }} \mathbb{C}_{4}$ and $\mathbb{C}_{2} \leq \mathbb{C}_{4}$. Observe that $\left(\mathrm{q}: \mathrm{op}: \mathrm{q}^{\prime}\right)$ is monotonic with respect to $\leq$ iff op is monotonic with respect to $\leq$. In addition, observe that if both op and $\mathrm{op}^{\prime}$ are monotonic, then so is $\mathrm{op} ; \mathrm{op}^{\prime}$. It is therefore enough to show monotonicity of nop, ct $\geq 1$, ct $:=c t+1$, ct $:=c t-1$ and grd $\Rightarrow$ cmd. The first four cases are straightforward. We show grd $\Rightarrow \mathrm{cmd}$ is monotonic. Suppose we are given $\mathbb{C}_{1}, \mathbb{C}_{2}, \mathbb{C}_{3}$ such that $\mathbb{C}_{1} \underset{\text { enc }\left(\text { abst }_{\Pi}(\operatorname{Pr})\right)}{\underset{\text { grd } \Rightarrow \text { cm }}{\longrightarrow}} \mathbb{C}_{2}$ and $\mathbb{C}_{1} \leq \mathbb{C}_{3}$. We exhibit a $\mathbb{C}_{4}$ such that $\mathbb{C}_{3} \underset{\text { enc }\left(\operatorname{abst}_{\Pi}(\operatorname{Pr})\right)}{\stackrel{\operatorname{grd} \Rightarrow \mathrm{cmd}}{\longrightarrow}} \mathbb{C}_{4}$ and $\mathbb{C}_{2} \leq \mathbb{C}_{4}$. The operation grd $\Rightarrow \mathrm{cmd}$, resulted from the assign rule in Figure 6.9. This was defined with respect to two pairs $\left(s^{b}, b^{b}\right)$ and $\left(s^{b^{\prime}}, b^{b^{\prime}}\right)$. We fix these two pairs. By the semantics of counter machines (Figure 6.8) and of the translation of the assign statement in Figure 6.9, the fact that $\mathbb{C}_{1} \underset{\operatorname{enc}\left(\operatorname{abst}_{\Pi}(\operatorname{Pr})\right)}{\stackrel{\operatorname{grd} \Rightarrow \mathrm{cmd}}{\longrightarrow}} \mathbb{C}_{2}$, ensures that there is a mapping $\mathbb{a}: A \rightarrow \mathbb{N}$ 
such that for any $\operatorname{ct}_{\left(\mathrm{pc}, 0_{p}^{b}\right)}$ we have $\mathbb{C}_{1}\left(\mathrm{ct}_{\left(\mathrm{pc},,_{p}^{b}\right)}\right)=\sum_{\left(0_{p}^{b}, 0_{p}^{b}\right) \in \operatorname{tf}} \mathbb{a}\left(a_{\left(\mathrm{pc}, 0_{p}^{b}, b_{p}^{\prime}{ }^{\prime}\right)}\right)$

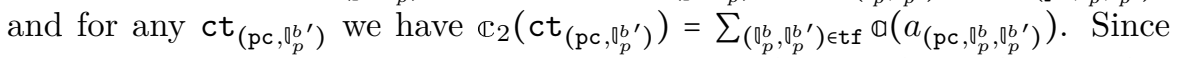
$\mathbb{C}_{1} \leq \mathbb{C}_{3}$, then for all $\mathrm{ct}_{\left(\mathrm{pc}, b_{p}^{b}\right)} \in \mathcal{C}$, we have that $\mathbb{C}_{3}\left(\mathrm{ct}_{\left(\mathrm{pc}, 0_{p}^{b}\right)}\right)=\mathbb{C}_{1}\left(\mathrm{ct}_{\left(\mathrm{pc}, 0_{p}^{b}\right)}\right)+$ $r_{\left(\mathrm{pc}, 0_{p}^{b}\right)}=\sum_{\left(0_{p}^{b}, b_{p}^{b}\right) \in \mathrm{tf}} \mathbb{a}\left(a_{\left(\mathrm{pc}, 0_{p}^{b}, b_{p}^{b}\right)}\right)+r_{\left(\mathrm{pc}, 0_{p}^{b}\right)}$ where $r_{\left(\mathrm{pc}, 0_{p}^{b}\right)} \geq 0$. The idea is to send these "excedents" along the enabled transfers. Fix such a $\left(\mathrm{pc}, 0_{p}^{b}\right)$. By definition of $\left(\mathbb{S}^{b}, \nabla^{b}, 0_{p}^{b}\right) \frac{\mathrm{v}_{1}^{b}, \ldots, \mathrm{v}_{n}^{b}:=\operatorname{ch}\left(\pi_{1}, \pi_{1}^{\prime}\right), \ldots, \operatorname{ch}\left(\pi_{n}, \pi_{n}^{\prime}\right)}{\operatorname{abst}_{\Pi}(\operatorname{Pr})}\left(\mathbb{S}^{b^{\prime}}, 0^{b^{\prime}}, 0_{p}^{b^{\prime}}\right)$ in Section 6.5.1 and of tf in Figure 6.9, we know there is at least one $\underline{0_{p}^{b^{\prime}}}$ such that $\left(\square_{p}^{b}, \underline{b_{p}^{\prime}}\right) \in \mathrm{tf}$. We define $\mathbb{C}_{4}\left(\mathrm{ct}_{\left(\mathrm{pc}, b_{p}^{b}\right)}\right):=\sum_{\left(\mathbb{b}_{p}^{b}, b_{p}^{\prime}\right) \in \mathrm{tf}} \mathbb{G}^{\prime}\left(a_{\left(\mathrm{pc}, b_{p}^{b}, b_{p}^{\prime}\right)}\right)$ where we have $\mathbb{a}^{\prime}\left(a_{\left(\mathrm{pc}, 0_{p}^{b}, v_{p}^{b^{\prime}}\right)}\right):=\mathbb{a}\left(a_{\left(\mathrm{pc}, 0_{p}^{b}, b_{p}^{b^{\prime}}\right)}\right)+r_{\left(\mathrm{pc}, 0_{p}^{b}\right)} \delta_{0_{p}^{b}, b_{p}^{\prime}}$ with $\delta_{0_{1}^{b}, b_{2}^{b}}$ iff $0_{1}^{b}$ is identical to $0_{2}^{b}$. So, $\mathbb{C}_{2}\left(\mathrm{ct}_{\left(\mathrm{pc}, 0_{p}^{b}\right)}\right) \leq \mathbb{C}_{4}\left(\mathrm{ct}_{\left(\mathrm{pc}, 0_{p}^{b}\right)}\right)$. We repeat the process for each counter $\operatorname{ct}_{\left(\mathrm{pc},,_{p}^{b}\right)}$ in $\mathcal{C}$. This results in a $\mathbb{C}_{4}$ where $\mathbb{C}_{2} \leq \mathbb{C}_{4}$ and for which the same transition (i.e. assign for the two pairs $\left(\Phi^{b}, 0^{b}\right)$ and $\left.\left(\mathbb{s}^{b^{\prime}}, 0^{b^{\prime}}\right)\right)$ is possible using the mapping $\mathbb{a}^{\prime}$.

In fact, even the rule target results in monotonic machine transitions for all counting predicates $\omega_{\text {trgt }}$ that denote upward closed sets of processes (since the intersection of two upward closeds is also upward closed). This is, for instance, the case for predicates capturing assertion violations but not for those capturing deadlocks (see Section 6.4). An encoding enc $\left(\operatorname{abst}_{\Pi}(\operatorname{Pr})\right.$ ) is said to be monotonic if all its transitions are monotonic. Checking assertion violations on abstractions obtained as in Section 6.5.1 always results in monotonic encodings.

Lemma 28 (decidability). State reachability of all monotonic encodings is decidable.

Proof. The ordering $\leq$ is a well-quasi-ordering on the set of configurations of enc $\left(\right.$ abst $\left._{\Pi}(\mathrm{Pr})\right)$ [48]. Monotonicity of the transition rules means the obtained counter machines result in well-structured transition systems $[2,59]$. It is known that state reachability is decidable for such systems [2].

Strengthening. Monotonic encodings correspond to coarse overapproximations. Intuitively, bad configurations (such as those in which a deadlock occurs, or those obtained in a backward exploration for a program with barriers such as the one in Figure 6.1) are no more guaranteed to be upward closed. This loss of precision makes verification out of the reach of techniques solely based on monotonic encodings. To regain some of the lost precision, we constrain the runs using counting invariants. This is done by strengthening the counter machine transitions in order to only allow configurations that are allowed by $\omega_{i n v}$. 
Example 14. Consider the concurrent program of Figure 6.1 with shared state $s=\{$ all $\leftarrow 4$, arrived $\leftarrow 0$, cross $\leftarrow 0$, read $\leftarrow 0\}$ and process configuration $\mathrm{m}=\left\{\mathrm{pc}_{3} \leftarrow 1, \mathrm{pc}_{1} \leftarrow 3\right\}$. After transition $\operatorname{tr}_{4}, \mathrm{~s}^{\prime}=$ $\{$ all $\leftarrow 4$, arrived $\leftarrow 1$, cross $\leftarrow 0$, read $\leftarrow 0\}$ and $\mathrm{m}^{\prime}=\left\{\mathrm{pc}_{4} \leftarrow 1, \mathrm{pc}_{1} \leftarrow 3\right\}$. Note that executing transition $\operatorname{tr}_{5}$ (barrier) at $\left(\mathrm{s}^{\prime}, \mathrm{m}^{\prime}\right)$ is impossible because of the barrier condition. Abstraction of the program with respect to the predicates $\pi_{1}=$ read_leq_0, $\pi_{2}=$ arrived_leq_all, $\pi_{3}=$ all_leq_arrived and $\pi_{4}=$ cross_leq_0 yields abstract transitions $\operatorname{tr}_{4}^{b}$ and $\operatorname{tr}_{5}^{b}$. It turns out that starting from the abstract configuration $\left(\mathrm{s}^{b}, \mathrm{~m}^{b}\right)$ the two transitions can be executed as follows:

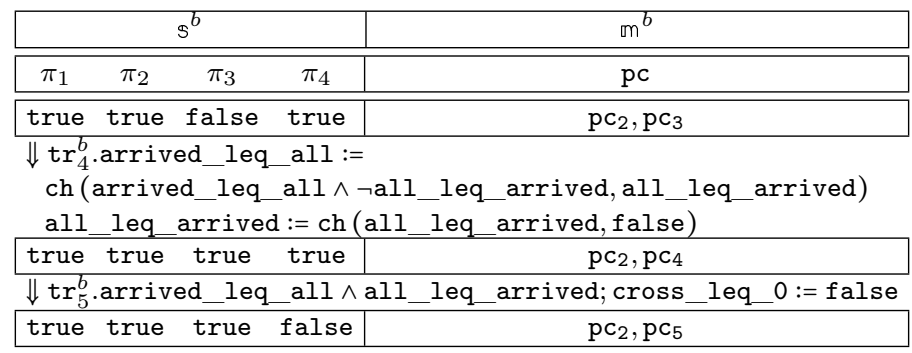

The steps above are not feasible in the original program, because $\operatorname{tr}_{4}^{b}$ changed predicate all_leq_arrived to true although all $\leq$ arrived does not hold in the original program. This results in a false positive.

Our solution to recover some of the lost precision is to strengthen the transitions of the counter machine using counting predicates (i.e., predicates that relate shared variables to the number of processes) that are valid in all runs (counting invariants), for example:

$$
\omega_{i n v}: \operatorname{all}=\sum_{i \geq 1}\left(@ p c_{i}\right)^{\#} \wedge \text { arrived }=\sum_{i \geq 4}\left(@ p c_{i}\right)^{\#} .
$$

Note that the counting invariant $\omega_{i n v}$ as opposed to the target predicates $\omega_{\text {trgt }}$ (Section 6.5.2) contains shared variables. This is to regain the information about the shared variables that was lost due to abstraction. Given an abstraction of a shared state $s^{b}$, we first conjunct $\omega_{i n v}$ with originof $\left(s^{b}\right)$ and project away the shared variables. We get a predicate that only contains counting variables. Then, we substitute the counting variables with their corresponding counters to obtain a predicate that only mentions counter machine variables. We finally strengthen all counter machine transitions that involve the state $\mathrm{q}_{s^{b}}$.

Example 15 (Strengthening). Consider the Boolean shared state $s^{b}$ with originOf $\left(s^{b}\right)=(($ read $\leq 0) \wedge($ arrived $\leq$ all $) \wedge($ all $\leq$ arrived $) \wedge \neg($ cross $\leq$ $0)$ ). After the conjunction with the invariant and projection of the shared variables, we obtain: $\exists$ Sh. $\left(\operatorname{originOf}\left(\mathrm{s}^{b}\right) \wedge \omega_{\text {inv }}\right)=\left(\left(@ \mathrm{pc}_{1}\right)^{\#}+\left(@ \mathrm{pc}_{2}\right)^{\#}+\right.$ $\left.\left(@ \mathrm{pc}_{3}\right)^{\#}\right)=0$. After substitution, we get $\operatorname{str}\left(\mathrm{s}^{b^{\prime}}, \omega_{\text {inv }}\right)=\left(\mathrm{ct}_{\left(\mathrm{pc}_{1}, \varnothing\right)}+\mathrm{ct}_{\left(\mathrm{pc}_{2}, \varnothing\right)}+\right.$ 


$$
\begin{aligned}
& \frac{\left(\mathrm{q}_{\mathrm{s}^{b}}: \mathrm{op}: \mathrm{q}_{\mathrm{s}^{b^{\prime}}}\right)}{\left(\mathrm{q}_{\mathrm{s}^{b}}: \operatorname{str}\left(\mathrm{s}^{b}, \omega_{i n v}\right) ; \mathrm{op} ; \operatorname{str}\left(\mathrm{s}^{b^{\prime}}, \omega_{i n v}\right): \mathrm{q}_{\mathrm{s}^{b^{\prime}}}\right)}
\end{aligned}
$$

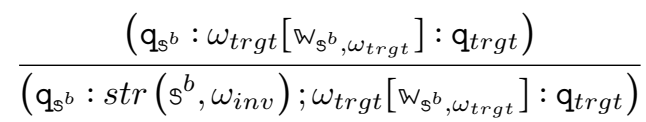

Figure 6.10: Strengthening of an extended counter machine transition using a counting invariant $\omega_{i n v}$. For this, let $\operatorname{str}\left(s^{b}, \omega_{i n v}\right)=$ $\left(\exists\right.$ Sh. $\left(\right.$ originOf $\left.\left.\left(\mathbb{S}^{b}\right) \wedge \omega_{i n v}\right)\right)\left[\mathfrak{W}_{\mathbb{S}^{b}, \omega_{i n v}}\right]$.

$\left.\mathrm{ct}_{\left(\mathrm{pc}_{3}, \varnothing\right)}=0\right)$. Each counter $\mathrm{ct}_{\left(\mathrm{pc}_{i}, \varnothing\right)}$ is the counter for the processes at location $\mathrm{pc}_{\mathrm{i}}$ where the processes have no local state. In other words, in the corresponding Boolean program, there exists no local or mixed predicates. We add the condition $\left(\mathrm{ct}_{\left(\mathrm{pc}_{1}, \varnothing\right)}+\mathrm{ct}_{\left(\mathrm{pc}_{2}, \varnothing\right)}+\mathrm{ct}_{\left(\mathrm{pc}_{3}, \varnothing\right)}=0\right)$ as a statement at the beginning (respectively, at the end) of each transition outgoing from (respectively, incoming to) $\mathrm{q}_{\mathrm{s}^{b}}$.

Strengthening is described in Figure 6.10. We need Lemma 29 and Lemma 30 in order to establish soundness of the strengthening step in Lemma 31. Lemma 29 states that including local and mixed predicates in the predicate abstraction step makes it possible to track the number of processes satisfying Boolean combinations of those predicates in the Boolean program abstraction.

Lemma 29 (abstraction and counting variables). If $\left(\mathrm{s}^{b}, \mathrm{~m}^{b}\right) \in \alpha((\mathrm{s}, \mathrm{m}))$ then for each predicate $\pi$ belonging to predsOf $f_{\text {exprsof }(\mathrm{Sh} \cup \mathrm{L})}$ s.t. atomsOf( $\left.\pi\right) \subseteq \Pi$, $\sum_{\{(\mathrm{pc}, 0) \mid \pi[\mathrm{s},(\mathrm{pc}, 0)]\}} \mathrm{m}((\mathrm{pc}, 0))=\sum_{\left\{\left(\mathrm{pc}, 0^{b}\right) \mid \pi\left[\mathrm{s}^{b},\left(\mathrm{pc}, 0^{b}\right)\right]\right\}} \mathrm{m}^{b}\left(\left(\mathrm{pc}, \mathbb{0}^{b}\right)\right)$.

Proof. By definition of $\alpha((\mathrm{s}, \mathrm{m})), \mathrm{m}^{b}[\mathrm{~s}, \mathrm{~m}]$ holds. This means that there is a bijection $h$ from $\mathrm{m}=<\sigma_{1}, \sigma_{2}, \ldots \sigma_{n}>$ to $\mathrm{m}^{b}=<\sigma_{1}^{\prime}, \sigma_{2}^{\prime}, \ldots \sigma_{n}^{\prime}>$ s.t. we can associate to each $\sigma_{i}=(\mathrm{pc}, 0)$ in $\mathrm{m}$ a $\sigma_{h(i)}=\left(\mathrm{pc}^{\prime}, 0^{b}\right)$ in $\mathrm{m}^{b}$ such that $\mathrm{pc}=\mathrm{pc}^{\prime}$ and $\nabla^{b}[\mathrm{~s}, 0]$. Let $\pi$ be a Boolean combination of predicates in $\Pi_{m i x} \cup \Pi_{l o c}$. By construction, we have that $\pi\left[\mathrm{s}^{b},\left(\mathrm{pc}, 0^{b}\right)\right] \Leftrightarrow \pi[\mathrm{s},(\mathrm{pc}, 0)]$. In fact, $\pi\left[\mathrm{s}^{b},\left(\mathrm{pc}, 0^{b}\right)\right]$ and $\pi[\mathrm{s},(\mathrm{pc}, 0)]$ coincide on each $\pi$ s.t. atoms $0 \mathrm{f}(\pi) \subseteq \Pi$. This implies that for $\pi$ with atomsOf $(\pi) \subseteq \Pi, \sum_{\{(\mathrm{pc}, 0) \mid \pi[\mathrm{s},(\mathrm{pc}, 0)]\}} \mathrm{m}(\mathrm{pc}, 0)=$ $\sum_{\left\{\left(\mathrm{pc}, 0^{b}\right) \mid \pi\left[\mathrm{s}^{b},\left(\mathrm{pc}, 0^{b}\right)\right]\right\}} \mathrm{m}^{b}\left(\mathrm{pc}, 0^{b}\right)$.

Lemma 30 (abstraction and strengthening). Assume $\left(\mathrm{s}^{b}, \mathrm{~m}^{b}\right) \in \alpha((\mathrm{s}, \mathrm{m}))$. If $\omega_{\text {inv }}[\mathrm{s}, \mathrm{m}]$ holds, then $\left(\exists \mathrm{Sh} .\left(\operatorname{originOf}\left(\mathrm{s}^{b}\right) \wedge \omega_{\text {inv }}\right)\right)\left[\mathrm{m}^{b}\right]$ also holds.

Proof. We know that $\omega_{i n v}[\mathrm{~s}, \mathrm{~m}]$ holds by assumption and originof $\left(\mathrm{s}^{b}\right)[\mathrm{s}]$ holds since $\left(\mathrm{s}^{b}, \mathrm{~m}^{b}\right) \in \alpha((\mathrm{s}, \mathrm{m}))$. Then, $\left(\right.$ origin0f $\left.\left(\mathrm{s}^{b}\right) \wedge \omega_{\text {inv }}\right)[\mathrm{s}, \mathrm{m}]$ also holds, and hence, the predicate $\left(\left(\operatorname{originOf}\left(s^{b}\right) \wedge \omega_{i n v}\right)[\mathrm{s}]\right)[\mathrm{m}]$. Since for each $(\pi)^{\#}$ appearing in $\operatorname{vars0f}\left(\omega_{i n v}\right)$, atomsOf $(\pi) \subseteq \Pi$, by Lemma 29 we can replace 
$m$ with $\mathrm{m}^{b}$ and obtain the predicate $\left(\left(\operatorname{origin0f}\left(\mathrm{s}^{b}\right) \wedge \omega_{\text {inv }}\right)[\mathrm{s}]\right)\left[\mathrm{m}^{b}\right]$. Finally, the existence of $s$ ensures that $\left(\exists \operatorname{Sh}\right.$. $\left(\right.$ originOf $\left.\left.\left(\mathrm{s}^{b}\right) \wedge \omega_{i n v}\right)\right)\left[\mathrm{m}^{b}\right]$ holds.

Lemma 31 (soundness of strengthening). If a $\operatorname{Pr}$ configuration satisfying $\omega_{\text {trgt }} \in$ predsOf $f_{\text {exprsof }\left(\Omega_{\mathrm{pc}, \mathrm{Sh}, \mathrm{L}}\right)}$ is reachable in $\operatorname{Pr}$, then $\mathrm{q}_{\text {trgt }}$ is reachable in enc $\left(a_{b s} t_{\Pi}(\operatorname{Pr})\right)$ after any strengthening with respect to some $\operatorname{Pr}$ invariant $\omega_{i n v} \in \operatorname{preds} O f_{\text {exprsof }\left(\mathrm{Sh} \cup \Omega_{\mathrm{pc}, \mathrm{Sh}, \mathrm{L}}\right)}$.

Proof. We proceed by contradiction. Assume a $\omega_{\text {trgt }}$ configuration is reachable in $\operatorname{Pr}$ while $\mathrm{q}_{\text {trgt }}$ is not reachable in a counter machine $\mathrm{M}$ obtained as the strengthening (with respect to a Pr counting invariant $\omega_{i n v}$ ) of an encoding of an abstraction abst $t_{\Pi}(\operatorname{Pr})$ of the program $\operatorname{Pr}$ as explained in Section 6.5.1. Let a feasible run $\rho_{\mathrm{Pr}}$ without an enc $\left(\text { abst }_{\Pi}(\operatorname{Pr})\right)_{s t r}$-feasible run $\rho_{\text {enc }\left(\text { abst }_{\Pi}(\operatorname{Pr})\right)_{s t r}}$ where enc $\left(\operatorname{abst}_{\Pi}(\mathrm{Pr})\right)_{s t r}$ is a strengthening of machine enc $\left(\right.$ abst $\left._{\Pi}(\operatorname{Pr})\right)$ with respect to an invariant $\omega_{i n v}$.

According to Definition 11, for each run $\rho_{\mathrm{Pr}}$, a non-empty set $\alpha\left(\rho_{\mathrm{Pr}}\right)$ of $\operatorname{abst}_{\Pi}(\operatorname{Pr})$-feasible runs exist. Moreover, based on Lemma 26, for each run $\rho_{\text {abst }_{\Pi}(\mathrm{Pr})} \in \alpha\left(\rho_{\mathrm{Pr}}\right)$ there exists an enc $\left(\operatorname{abst}_{\Pi}(\mathrm{Pr})\right)$-feasible run $\rho_{\text {enc }\left(\mathrm{abst}_{\Pi}(\mathrm{Pr})\right)}$ (before strengthening). So, if the run $\rho_{\text {enc }\left(\operatorname{abst}_{\Pi}(\operatorname{Pr})\right)_{s t r}}$ does not exist, it is because the run $\rho_{\text {enc }\left(\text { abst }_{\Pi}(\mathrm{Pr})\right)}$ was not possible after the strengthening phase.

Let $\rho_{\text {enc }\left(\mathrm{abst}_{\Pi}(\mathrm{Pr})\right)}=\left(\mathrm{q}_{\mathrm{s}_{0}^{b}}, \mathbb{C}_{\mathrm{m}_{0}^{b}}\right) ; \mathrm{t}_{1} ; \ldots ;\left(\mathrm{q}_{\mathrm{s}_{n}^{b}}, \mathbb{C}_{\mathrm{m}_{n}^{b}}\right)$ and $\rho_{\mathrm{Pr}}=$ $\left(s_{0}, \mathrm{~m}_{0}\right) ; \operatorname{tr}_{1} ; \ldots ;\left(\mathrm{s}_{n}, \mathrm{~m}_{n}\right)$ with $\left(\mathrm{s}_{i}^{b}, \mathrm{~m}_{i}^{b}\right) \in \alpha\left(\left(\mathrm{s}_{i}, \mathrm{~m}_{i}\right)\right)$ for each $i: 0 \leq i \leq n$. Because $\rho_{\text {enc }\left(\mathrm{abst}_{\Pi}(\mathrm{Pr})\right)}$ is removed after strengthening, then there exists a step $\left(\mathrm{q}_{s^{b}}, \mathbb{C}_{\mathrm{m}^{b}}\right) \underset{\operatorname{enc}\left(\mathrm{abst}_{\Pi}(\mathrm{Pr})\right)}{\left(\mathrm{q}_{\mathrm{s}^{b}} \mathrm{op:} \mathrm{q}_{\mathrm{s}^{\prime}}\right)}\left(\mathrm{q}_{\mathrm{s}^{b^{\prime}}}, \mathbb{C}_{\mathrm{m}^{b}}^{\prime}\right)$ in $\rho_{\text {enc }\left(\operatorname{abst}_{\Pi}(\mathrm{Pr})\right)}$ such that its corresponding step is impossible. According to the strengthening rule in Figure $6.10, \operatorname{str}\left(\AA^{b}, \omega_{i n v}\right)=\left(\exists\right.$ Sh.originOf $\left.\left(\AA^{b}\right) \wedge \omega_{i n v}\right)\left[\mathrm{w}_{s^{b}, \omega_{i n v}}\right]$. So, the fact that the mentioned step is not possible after strengthening implies that either $\left(\mathrm{q}_{\mathrm{s}^{b}}, \mathbb{C}_{\mathrm{m}^{b}}\right)$ does not satisfy $\operatorname{str}\left(\mathrm{s}^{b}, \omega_{i n v}\right)$, or that $\left(\mathrm{q}_{\mathrm{s}^{b^{\prime}}}, \mathbb{C}_{\mathrm{m}^{b^{\prime}}}\right)$ does not satisfy $\operatorname{str}\left(\mathrm{s}^{b^{\prime}}, \omega_{i n v}\right)$. Both alternatives violate the fact that $\omega_{i n v}$ is an invariant, that $(s, m)$ and $\left(s^{\prime}, m^{\prime}\right)$ are both reachable, and Lemma 30 .

The resulting machine is not monotonic in general and we can encode the state reachability of a 2-counter Minsky machine.

Lemma 32. State reachability is in general undecidable after strengthening.

Proof. Let $\mathrm{M}=\left(\mathcal{Q}, \mathcal{C}, \mathrm{T}, \mathcal{Q}_{\text {init }}, \mathbb{C}_{\text {init }}, \mathrm{q}_{\text {trgt }}\right)$ be an arbitrary 2-counter Minsky machine as introduced in Section 3.2.2. We describe a concurrent program in Figure 6.11 together with predicates $\Pi_{0}$, a counting invariant $\omega_{i n v}$ and a counting predicate $\omega_{\text {trgt }}$. The obtained strengthened encoding captures the counter machine $\mathrm{M}$ in the sense that solving the reachability of the target state of the encoding is equivalent to checking reachability of $\mathrm{q}_{\text {trgt }}$ for $\mathrm{M}$, which is undecidable in general. 
Each process of the concurrent program manipulates a local variable tl and the shared variables $c t_{1}, c t_{2}, s t$, and $t s$. The local variable $t l$ ranges over the three distinct constants $k_{\mathrm{pm}}, \mathrm{k}_{\mathrm{p} 1}, \mathrm{k}_{\mathrm{p} 2}$. These are used to identify three types of process instances. The shared variable ts ranges over the constants in $\left\{\mathrm{k}_{\mathrm{pm}}, \mathrm{k}_{\mathrm{p} 1}, \mathrm{k}_{\mathrm{p} 2}, \operatorname{dec}_{1}, \operatorname{dec}_{2}\right\}$. Exactly one instance (called the main instance, henceforth) of the processes executes transitions starting with the guard $\mathrm{ts}=\mathrm{k}_{\mathrm{pm}}$. The main instance spawns (and joins with) two kinds of auxiliary processes, those that execute the transition with the assumption that $\mathrm{tl}=\mathrm{ts}=\mathrm{k}_{\mathrm{p} 1}$ and those that execute the transition when $\mathrm{tl}=\mathrm{ts}=\mathrm{k}_{\mathrm{p} 2}$. Henceforth, these will be referred to as $k_{\mathrm{p} 1}$ and $k_{\mathrm{p} 2}$ instances. The variable st ranges over $|\mathcal{Q}|$ distinct constants $\left\{\mathrm{k}_{\mathrm{q}} \mid \mathrm{q} \in \mathcal{Q}\right\}$ and encodes the state $\mathrm{q} \in \mathcal{Q}$ of the counter machine M. Finally, the variable $c_{1}$ is used to capture the number of $\mathrm{k}_{\mathrm{p} 1}$ instances, and $c \mathrm{t}_{2}$ the number of $\mathrm{k}_{\mathrm{p} 2}$ instances. Figure 6.11 represents the encoding of operations on $c t_{1}$. Encoding of $c t_{2}$ operations is similar.

Finally, we let $\Pi_{0}=\left\{\mathrm{ct}_{1}=0, \mathrm{ct}_{2}=0\right\} \cup\left\{\mathrm{st}=\mathrm{k}_{\mathrm{q}} \mid \mathrm{q} \in \mathcal{Q}\right\}, \omega_{\text {trgt }}=$ $\left(\mathrm{st}=\mathrm{k}_{\mathrm{trgt}}\right)^{\#} \geq 1$ and:

$$
\omega_{i n v}=\left(\begin{array}{l}
\left(@ p c_{x}\right)^{\#} \leq 1 \\
\wedge\left(c t_{1}=\left(@ p c_{1} \wedge t 1=k_{\mathrm{p} 1}\right)^{\#}\right) \\
\left.\wedge\left(c t_{2}=\left(@ p c_{1} \wedge t 1=k_{\mathrm{p} 2}\right)^{\#}\right)\right)
\end{array}\right)
$$

Predicate abstraction will maintain the predicates $\mathrm{ct}_{i}=0$, but will lose their connection with the number of the corresponding instances. Strengthening re-establishes this connection and introduces tests for zero on the counters tracking $\mathrm{k}_{\mathrm{p} 1}, \mathrm{k}_{\mathrm{p} 2}$ instances, exactly at the encodings of the transitions $\operatorname{tr}_{t s t}$.

\subsubsection{Constrained monotonic abstraction and preorder refinement}

This step addresses the state reachability problem for an extended counter machine $\mathrm{M}=\left(\mathcal{Q}, \mathcal{C}, \mathrm{T}, \mathcal{Q}_{\text {init }}, \mathbb{C}_{\text {init }}, \mathrm{q}_{\text {trgt }}\right)$. As stated in Lemma 32 , this problem is undecidable in general for strengthened encodings. The idea of monotonic abstraction [4] as explained in Section 3.4 is to force monotonicity with respect to a well-quasi-ordering $\leq$ on the set of machine configurations. We adapt the backward reachability analysis procedure "explore" introduced in Section 3.3. An extended counter machine $\mathrm{M}=\left(\mathcal{Q}, \mathcal{C}, \mathrm{T}, \mathcal{Q}_{\text {init }}, \mathbb{C}_{\text {init }}, \mathrm{q}_{\text {trgt }}\right)$ induces the infinite-state transition system $(\mathrm{C}, \mathrm{T}, \rightarrow)$ where $\mathrm{C}$ is the set of configurations. A configuration is a tuple $(\mathrm{q}, \mathbb{C}) . \rightarrow$ is the transition relation defined in figure 6.8. We start with the preorder $\leq_{0} \subseteq \mathrm{C} \times \mathrm{C}$ such that for $\mathrm{c}=(\mathrm{q}, \mathbb{C})$ and $\mathrm{c}^{\prime}=\left(\mathrm{q}^{\prime}, \mathbb{c}^{\prime}\right)$, $c \leq c^{\prime}$ iff $(1) q=q^{\prime}$, and $(2) \mathbb{C} \leq \mathbb{C}^{\prime}$ with respect to the natural component-wise ordering defined as $\underset{\mathrm{ct} \in \mathcal{C}}{\wedge} \mathbb{C}(\mathrm{ct}) \leq \mathbb{C}^{\prime}(\mathrm{ct})$. Intuitively, $\mathbb{C} \leq_{0} \mathbb{C}^{\prime}$ holds if $\mathbb{C}^{\prime}$ can be 


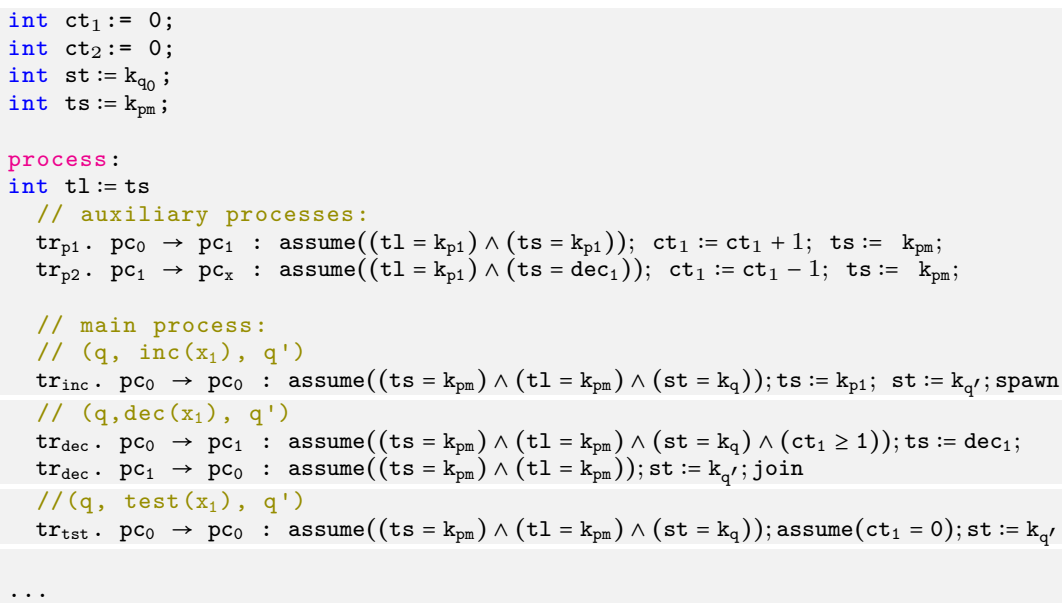

Figure 6.11: Encoding a 2-counter Minsky machine for strengthened counter machines obtained as encoding of Boolean concurrent programs.

obtained by "adding more processes to" $\mathbb{C}$. Using this ordering results in a WSTS. The procedure "explore" requires that we can compute membership, upward closure, entailment with respect to the preorder $\leq$, and predecessor computations of an upward closed set. As explained in Example 1 in Section 3.3 , the procedure can work with upward closed sets.

If no run is found, then not_reachable is returned. We modify the algorithm slightly so that upon finding a run at line 5 , the result of the counterexample analyzer procedure introduced in Section 3.4.1, "simulate $(\mathrm{M}, \leq, \rho)$ " is returned. The counterexample analyzer simulates the obtained run on the concrete machine M. If the run is possible, it is sent to the fourth step of our approach (described in Section 6.5.5). Otherwise, the upward closure $\operatorname{Up}_{\leq}((q, \mathbb{C}))$ responsible for the spurious trace is identified and an interpolant $I$ (with $\operatorname{varsOf}(I) \subseteq \mathcal{C}$ ) is used to refine the preorder as follows: $\leq_{i+1}:=\left\{\left((\mathrm{q}, \mathbb{C}),\left(\mathrm{q}, \mathbb{C}^{\prime}\right)\right) \mid(\mathrm{q}, \mathbb{C}) \unlhd_{i}(\mathrm{q}, \mathbb{C}) \wedge\left(\mathbb{C} \vDash I \Leftrightarrow \mathbb{C}^{\prime} \vDash I\right)\right\}$.

Although stronger, the new preorder is again a well-quasi-ordering and the trace is guaranteed to be eliminated in the next round. This is explained in detail in Chapter 7.

Lemma 33 (CMA [3]). All steps involved in the procedure "explore" are effectively computable and each instantiation of the procedure is sound and terminates given the preorder is a well-quasi-ordering. 


\begin{tabular}{|c|c|c|c|c|c|c|c|}
\hline \multirow[b]{2}{*}{ example } & \multirow[b]{2}{*}{$\operatorname{Pr}$} & \multirow[b]{2}{*}{$\operatorname{enc}\left(\operatorname{abst}_{\Pi}(\mathrm{Pr})\right)$} & \multicolumn{2}{|c|}{ outer loop } & \multicolumn{2}{|c|}{ inner loop } & \multirow[b]{2}{*}{$\operatorname{time}(\mathrm{s})$} \\
\hline & & & num. & preds. & num. & preds. & \\
\hline \multirow{3}{*}{ cyclic barrier } & \multirow{3}{*}{$5: 3: 12$} & $13: 31: 79$ & 4 & 5 & 5 & 1 & 30 \\
\hline & & $13: 4: 35$ & 2 & 2 & 2 & 0 & 15 \\
\hline & & 13:8:64 & 4 & 3 & 6 & 2 & 35 \\
\hline \multirow{3}{*}{ dynamic barrier } & \multirow{3}{*}{$5: 2: 8$} & $8: 8: 44$ & 3 & 3 & 3 & 0 & 20 \\
\hline & & $8: 1: 14$ & 2 & 1 & 2 & 0 & 3 \\
\hline & & $8: 1: 8$ & 1 & 0 & 1 & 0 & 1 \\
\hline \multirow{3}{*}{ flag } & \multirow{3}{*}{$5: 2: 9$} & $8: 16: 123$ & 4 & 4 & 6 & 2 & 34 \\
\hline & & $8: 8: 67$ & 3 & 2 & 3 & 0 & 5 \\
\hline & & $8: 4: 22$ & 3 & 2 & 5 & 2 & 18 \\
\hline \multirow{3}{*}{ semaphore } & \multirow{3}{*}{$4: 2: 7$} & $7: 32: 89$ & 5 & 5 & 5 & 0 & 68 \\
\hline & & $7: 7: 24$ & 3 & 3 & 3 & 0 & 14 \\
\hline & & $7: 16: 66$ & 4 & 4 & 6 & 2 & 17 \\
\hline \multirow{3}{*}{ maximum } & \multirow{3}{*}{$5: 2: 8$} & $18: 16: 172$ & 5 & 5 & 8 & 3 & 489 \\
\hline & & 18:4:51 & 3 & 3 & 3 & 0 & 18 \\
\hline & & $8: 8: 48$ & 4 & 3 & 6 & 2 & 21 \\
\hline \multirow{3}{*}{ parent-child } & \multirow{3}{*}{$2: 3: 10$} & $9: 16: 48$ & 3 & 4 & 5 & 2 & 76 \\
\hline & & $9: 1: 16$ & 2 & 1 & 2 & 0 & 2 \\
\hline & & $9: 4: 35$ & 3 & 2 & 3 & 0 & 5 \\
\hline \multirow{3}{*}{ as-many } & \multirow{3}{*}{$3: 2: 6$} & $8: 4: 34$ & 3 & 2 & 6 & 3 & 68 \\
\hline & & $8: 1: 9$ & 2 & 1 & 2 & 0 & 2 \\
\hline & & $8: 2: 8$ & 2 & 1 & 2 & 0 & 2 \\
\hline \multirow{3}{*}{ locals } & \multirow{3}{*}{$6: 3: 13$} & $14: 8: 47$ & 3 & 3 & 3 & 0 & 16 \\
\hline & & $14: 2: 24$ & 2 & 1 & 2 & 0 & 8 \\
\hline & & $14: 16: 95$ & 4 & 4 & 4 & 0 & 58 \\
\hline \multirow{3}{*}{ shareds } & \multirow{3}{*}{$7: 3: 11$} & $13: 32: 130$ & 4 & 5 & 10 & 6 & 160 \\
\hline & & $13: 2: 21$ & 2 & 1 & 2 & 0 & 6 \\
\hline & & $12: 2: 20$ & 2 & 1 & 2 & 0 & 17 \\
\hline
\end{tabular}

Table 6.1: The results of checking assertion violation and deadlock with PACMAN

\subsubsection{Simulation on the original concurrent program}

A run of the counter machine $\left(\mathcal{Q}, \mathcal{C}, \mathrm{T}, \mathcal{Q}_{\text {init }}, \mathbb{C}_{\text {init }}, \mathrm{q}_{\text {trgt }}\right)$ is simulated by this step on the original concurrent program $\operatorname{Pr}=(\mathrm{Sh}, \mathrm{L}, \mathrm{Tr})$. This is possible because each step of the counter machine run corresponds to a unique and concrete transition of $\mathrm{Pr}$. This step is classical in counterexample-guided abstraction refinement approaches. In our case, we need to differentiate the variables belonging to different processes during the simulation. As usual in such frameworks, if the trace turns out to be possible, then we have captured a concrete run of $\mathrm{Pr}$ that violates an assertion, and we report it. Otherwise, we deduce predicates that make the run infeasible (Sect. 6.5.1). 
Theorem 21 (predicated constrained monotonic abstraction). Assume an effective and sound predicate abstraction. If the constrained monotonic abstraction step returns not_reachable, then no configuration satisfying $\omega_{\text {trgt }}$ is reachable in $\operatorname{Pr}$. If a $\operatorname{Pr}$ run is returned by the simulation step, then it reaches a configuration where $\omega_{\text {trgt }}$ holds. Every iteration of the outer loop (predicate abstraction refinement) terminates given that the inner loop (monotonic abstraction refinement) terminates. Every iteration of the inner loop terminates.

Proof. By Lemma 25 and Lemma 29, if the concretization of a run of an encoding is Pr-feasible, then $\omega_{\text {trgt }}$ is Pr-reachable. Soundness is given by soundness of the predicate abstraction and strengthening (Lemma 31) and soundness of the CMA step (Lemma 33). Termination of each iteration of the inner loop is by well-quasi-ordering (Lemma 33). Termination of each iteration of the outer loop, given that the inner one terminates, is by effectiveness of the predicate abstraction (Definition 11) and construction of the encoding (Figure 6.9) and strengthening (Figure 6.10).

Notice that there is no general guaranty that we establish or refute the safety property (the problem is undecidable). For instance, it may be the case that one of the loops does not terminate (although each one of their iterations does) or that we need to add predicates that relate the local variables of two different processes (something the predicate abstraction framework we use cannot express).

\subsection{Experimental results}

We report on experiments with our open-source tool prototype PACMAN (for

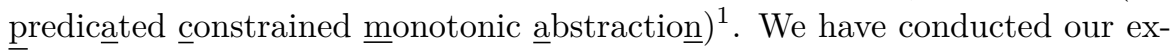
periments on an Intel Core(TM) i7 processor running at $2.9 \mathrm{GHz}$ with $8 \mathrm{~GB}$ of memory. The reported examples that require refinements of the natural preorder are challenging for existing techniques, either because the examples require stronger orderings than the usual preorder, or because they involve counting target predicates that are not expressed in terms of violations of program assertions. All predicate abstraction predicates and counting invariants have been derived automatically. For the counting invariants, we implemented a thread-modular analysis operating on the polyhedra numerical domain. We make use of several optimizations as explained in the following.

- Invariants and unsatisfiable combinations. We discard Boolean mappings corresponding to unsatisfiable combinations of predicates, for example $\neg($ arrived $\leq$ all $) \wedge \neg($ all $\leq$ arrived $)$ in Figure 6.1. We also use automatically generated invariants (such as (arrived $\leq$ all) $\wedge$

\footnotetext{
${ }^{1}$ https://gitlab.liu.se/live/pacman
} 
(arrived $\geq 0$ ) in the same example) to filter the state space. Such heuristics dramatically help our state space exploration algorithm.

- Auxiliary variables. To make the analysis possible, we try to use as few auxiliary variables as possible. An auxiliary variable $a_{\left(\mathrm{pc}, 1^{b}, 1^{b^{\prime}}\right)}$ captures how many processes in local state $\left(\mathrm{pc}, 1^{b}\right)$ will move to state $\left(\mathrm{pc}, 1^{b^{\prime}}\right)$ because of the broadcast transition due to a multiple assignment. Each time this number is constant, which turns out to happen often, we discard the auxiliary variable $a_{\left(\mathrm{pc}, 1^{b}, 1^{b^{\prime}}\right)}$.

For each example, we give the number of transitions and variables both in Pr and in the resulting counter machine.

The tuples under the Pr column of Table 6.1 respectively refer to the size of the original program, i.e., number of variables, procedures and transitions in the original program. The tuples under the enc $\left(\right.$ abst $\left._{\Pi}(\operatorname{Pr})\right)$ column refer to the size of the counter machine, i.e., number of counters, states and transitions in the extended counter machine. We also state the number of refinement steps and predicates automatically obtained in both refinement loops. We mention the required total time for the analysis. The tool and examples are accessible $^{2}$.

We report on experiments checking assertion violation and deadlock freedom in Table 6.1. For each example, we check three versions: the first one is correct and PACMAN establishes that, the second one is modified so that an assertion can be violated, and the third one is modified so that deadlock is possible. for the latter two cases, PACMAN exhibits faulty runs as expected.

\subsection{Conclusions}

We have presented a technique, predicated constrained monotonic abstraction, for the automated verification of concurrent programs whose correctness depends on synchronization between arbitrarily many processes, for example by means of barriers implemented using integer counters and tests. We have introduced counting predicates and used them in a framework that combines predicate, counter and constrained monotonic abstraction. Our prototype implementation gave encouraging results and managed to automatically establish or refute program assertions and deadlock freedom. Chapter 5 showed that barriers could be modeled using phasers and explained that checking reachability of phaser programs with arbitrarily many tasks is decidable if the phaser program does not have atomic statements. However, in this chapter, we proved that checking reachability of strengthened counter programs that model barriers is undecidable. These two results do not contradict each other. The intuition is that strengthening and atomic transitions in the obtained boolean programs of this chapter allow to relate tests for zero to the

\footnotetext{
${ }^{2}$ https://gitlab.liu.se/live/pacman
} 
number of processes (barriers) and atomic transitions (by enforcing that only a single process can run a sequence of instructions).

In Chapter 7 we investigate how to improve scalability of the constraint monotonic abstraction approach used in this chapter by lazily refining monotonic abstraction. 


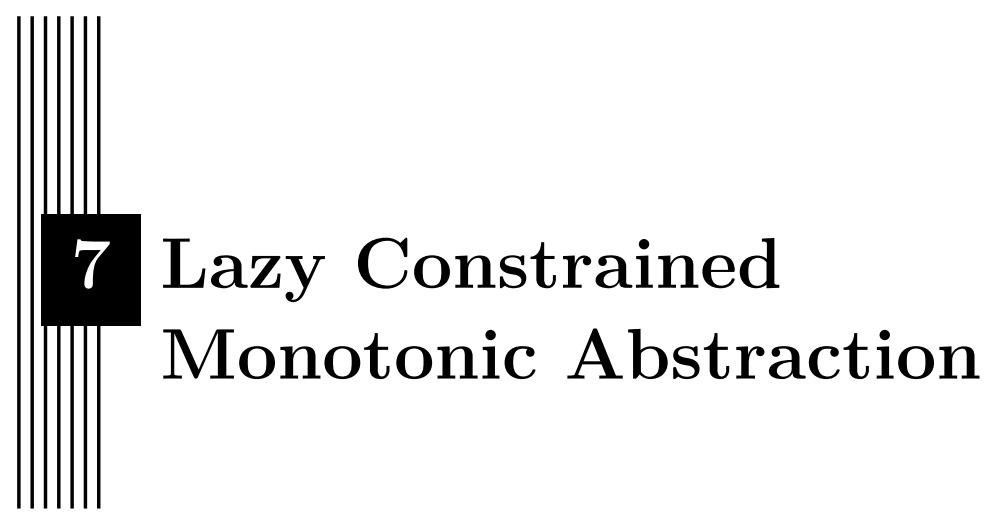

In this chapter, we introduce Lazy Constrained Monotonic Abstraction (Lazy CMA for short) for lazily and soundly exploring well-structured abstractions of infinite and non-monotonic transition systems. Monotonic abstraction was used in the verification approach that was introduced in Chapter 6. We consistently improve the monotonic abstraction approach by localizing refinements and by partially preserving the explored state space each time a refinement step is required for the ordering. For this purpose, we adapt ideas from the classical lazy predicate abstraction.

\subsection{Introduction}

Well-structured transition systems (WSTS:s for short) may be everywhere [59], but not all transition systems are well-structured [3, 61]. Problems such as state reachability (e.g, safety) have been shown to be decidable for WSTS:s $[2,59]$. This led to the development of algorithms that could check safety for systems ranging from lossy channels and Petri Nets to concurrent programs and broadcast protocols $[63,82,96]$. Many interesting examples of such systems, including list manipulating programs [26], cache coherence protocols [47] and mutex algorithms [5] are "almost" well-structured in the sense that they would have been well-structured if it was not for a number of transitions that violate the required assumptions. We build on the framework of Constrained Monotonic Abstraction (CMA for short) [3] where we derive well-structured abstractions for infinite-state systems that are "almost" well-structured. 
Systems where only some transitions are non-monotonic can be approximated using WSTS:s by adding abstract transitions to restore monotonicity (monotonic abstraction). The resulting abstraction is also infinite-state, and reachability of its upward-closed sets is decidable. However, the obtained abstractions may fail to enforce invariants that are crucial for establishing unreachability of bad configurations in the original system. We explained in Chapter 6 how we automatically account for the number of processes synchronizing with (dynamic) barriers when establishing or refuting local (e.g., assertions) and global (e.g., deadlock freedom) properties of concurrent programs that manipulate arbitrarily many processes. Crucial invariants of such systems enforce an inherently non-monotonic behavior (e.g., a barrier transition that is enabled on a configuration is disabled if more processes are considered in a larger configuration).

Checking safety for such non-monotonic systems is not guaranteed to terminate without abstraction. Plain monotonic abstraction [5, 65] makes use of sets that are upward-closed with respect to natural orderings as a sound symbolic representation. As stated earlier, this ensures termination if the preorder that is used is a $\mathcal{W} \mathcal{Q O}[2]$. Of course, this comes at the price of possible false positives. In [3], the authors adapted the existing counterexampleguided abstraction refinement (CEGAR) ideas to refine the ordering in plain monotonic abstraction. The preorder is strengthened by only relating configurations that happen to be in the same equivalence class, as defined by the Craig interpolants obtained from the false positives. The new preorder is also a $\mathcal{W} \mathcal{Q O}$, and hence, termination is again ensured. As implemented, the predicates are applied on all generated minimal elements to separate upwardclosed sets and the exploration has to restart from scratch each time a new refinement predicate is encountered.

We address these inefficiencies by adopting a lazy approach. Like in lazy predicate abstraction [71], we strive to localize the application of the refinement predicates and to reuse the explored state space. However, a major difference with lazy predicate abstraction is that the number of "control locations" (i.e., the locations to which subsets of the refinement predicates are mapped) is a priori unbounded (as opposed to the number of program locations of a non-parameterized system). In this chapter, we propose three heuristics that can be applied both in backward and forward (something plain monotonic abstraction is incapable of). All three heuristics adopt a backtracking mechanism to reuse, as much as possible, the state space that has been explored so far. Schematically, the first heuristic (point-based) associates refinement predicates to minimal elements. The second heuristic (order-based) associates the refinement predicates to preorder-related minimal elements. The third heuristic (descendants-based) uses for a child the preserved predicates of its parent. We describe in detail the different approaches and state the soundness and termination of each refinement step. In addition, we experimentally 
compare the heuristics against each other and against the eager approach (CMA) on our open-source tool https://gitlab.liu.se/live/zaama.

Related work. Coverability of non-monotonic systems is undecidable in general. Tests for zero are one source of non-monotonicity. The work in [25] introduces a methodology for checking coverability by using an extended Karp-Miller acceleration for the case of Vector Addition Systems (VAS:s for short) with at most one test for zero. Our approach is more general and tackles coverability and reachability for counter machines with arbitrary tests.

Verification methods can be lazy in different ways. For instance, Craig interpolants obtained from program runs can be directly used as abstractions [102], or abstraction predicates can be lazily associated to program locations [71]. Such techniques are now well established [15, 38, 127]. Unlike these approaches, we address lazy exploration for transition systems with "infinite control". The existing abstract WSTS:s that do not allow to refine the ordering being used [82, 96], cannot model transfers for the local variables [58], or make use of accelerations without termination guarantees [18]. For example, in [82] the authors leverage the combination of an exact forward reachability and of an aggressive backward approximation, while in [96], the explicit construction of a Petri Net is avoided.

The work in [85] gives a generalization of the IC3 algorithm and tries to build inductive invariants for well-structured transition systems. There is no straightforward approach to adapt it to the kind of non-monotonic systems that we work with.

We believe the approach proposed here can be combined with such techniques. To the best of our knowledge, there is no previous work that considered making the preorder refinement of a WSTS abstraction lazy.

Outline. We start in Section 7.2 with some preliminaries. We describe the adopted symbolic representation in Section 7.3 and go through a illustrative example in Section 7.4. This is followed by a description of the eager and lazy procedures in Section 7.5. We finally report on our experiments in Section 7.6 and conclude in Section 7.7.

\subsection{Preliminaries}

For a set of integer variables V, we write atoms $0 f(V)$ to mean the set of atoms over the variables V. An atom $\alpha$ is either a Boolean true or false or an inequality $\mathrm{e} \sim \mathrm{e}^{\prime}$ of two arithmetic expressions; where $\sim \in\{<, \leq, \geq,>\}$. We write A to mean a set of atoms. Observe that the negation of an atom can be expressed as an atom. We often write $\psi$ to mean a conjunction of atoms, or conjunct for short, and use $\Psi$ to mean a set of conjuncts. We use predsOf $f_{\text {exprsof(v) }}$ to mean arbitrary conjunctions and disjunctions of atoms 
over the set of integer variables V. We can rewrite any Presburger predicate over $\mathrm{V}$ in negated normal form and replace the negated inequalities with the corresponding atoms to obtain an equivalent predicate $\pi$ in preds $0 f_{\text {exprsof(v) }}$.

A mapping $x: U \rightarrow V$ associates an element in $V$ to each element in $U$. We write $x: U \rightarrow V$ to mean a partial mapping from $U$ to $V$. We write $\operatorname{dom}(x)$ and image $(x)$ to respectively mean the domain and the image of $x$ and use $\epsilon_{U}: U \rightarrow V$ for the mapping with an empty domain. We often write a partial mapping $\mathfrak{x}: U \rightarrow V$ as the set $\{u \leftarrow \mathfrak{x}(u) \mid u \in \operatorname{dom}(\mathfrak{x})\}$ and write $\mathfrak{x} \cup \mathfrak{x}^{\prime}$ to mean the union of two mappings $x$ and $x^{\prime}$ with disjoint domains. Given a partial mapping $\vee: X \rightarrow \operatorname{exprs0f}(X)$, we write $\mathrm{e}[\mathrm{v}]$ to mean the substitution of $\mathrm{V}$ variables in e by their respective $\vee$ images and the natural evaluation of the result. As usual, e[v] is a well defined integer value each time $\vee$ is a total mapping to $\mathbb{Z}$. This is generalized to (sets of) atoms, conjuncts and predicates.

We let $\mathbb{V}$ (resp. $\left.\mathbb{V}_{\geq 0}\right)$ be the set of all total mappings $\mathrm{V} \rightarrow \mathbb{Z}($ resp. $\mathrm{V} \rightarrow \mathbb{N})$. We write $\mathbb{O}_{\mathrm{V}}$ for the total mapping $\mathrm{V} \rightarrow\{0\}$. The denotation of a conjunct $\psi$ over $\mathbb{V}$ (resp. $\left.\mathbb{V}_{\geq 0}\right)$, written $\left.\llbracket \psi\right]_{\mathbb{V}}$ (resp. $\left[[\psi] \mathbb{V}_{\geq 0}\right)$, is the set of all total mappings $\vee$ in $\mathbb{V}$ (resp. in $\left.\mathbb{V}_{\geq 0}\right)$ s.t. $\psi[\mathbb{v}]$ evaluates to true. We generalize to sets of atoms or conjuncts by taking the union of the individual denotations. Let $\unlhd$ be the preorder over $\mathbb{V}_{\geq 0}$ defined by $\vee \unlhd v^{\prime}$ iff $\nabla(v) \leq \nabla^{\prime}(v)$ for each integer variable $\mathrm{v} \in \mathrm{V}$. Given a predicate $\psi$ in preds $0 f_{\text {exprsof }(\mathrm{v})}$, we say that a set $M \subseteq\left[[\psi] \mathbb{v}_{\geq 0}\right.$ is minimal for $\psi$ if: (i) $\vee \Varangle \nabla^{\prime}$ for any pair of different $\vee, \nabla^{\prime} \in M$, and (ii) for any $\nabla^{\prime} \in\left[[\psi] \mathbb{v}_{\geq 0}\right.$, there is a $\vee \in M$ s.t. $\vee \unlhd v^{\prime}$. We recall the following facts from Linear Programming and [72].

Lemma 34. For a finite set of natural variables $\mathrm{V}$, the preorder $\unlhd$ is a wellquasi-ordering. In addition, we can compute a finite and unique minimal set (written $\operatorname{Min}_{\unlhd}(\pi)$ ) for any predicate $\pi$ in predsOf $f_{\text {exprsof }(\mathrm{v})}$.

\subsection{Symbolic representation}

Assume an extended counter machine $\left(\mathcal{Q}, \mathcal{C}, \mathrm{T}, \mathcal{Q}_{\text {init }}, \mathbb{C}_{\text {init }}, \mathrm{q}_{\text {trgt }}\right)$ as introduced in Chapter 6. We introduce the symbolic representations used in our reachability procedures in Section 7.5.

Boxes. A box $\mathrm{b}$ over a set $\mathrm{A}$ of atoms is a partial mapping from A to Booleans B. Intuitively, a box corresponds to a bitvector denoting an equivalence class in the classical predicate abstraction. We use it to constrain the upward closure. The predicate $\psi_{\mathfrak{b}}$ of a box $\mathbb{b}$ is $\wedge_{\alpha \in \operatorname{dom}(\mathfrak{b})}((\mathfrak{b}(\alpha) \wedge \alpha) \vee(\neg \mathbb{b}(\alpha) \wedge \neg \alpha))$ (true is used for the empty box). Observe that this predicate is indeed a conjunct for any fixed box $\mathbb{b}$ and that $\left[\left[\psi_{\mathfrak{b}}\right]\right]$ does not need to be finite. We write $\mathfrak{b}_{\text {true }}$ for the box of the true conjunct. We will say that a box $\mathbb{b}$ is weaker than (or is entailed by) a box $\mathbb{b}^{\prime}$ if $\psi_{\mathfrak{b}^{\prime}} \Rightarrow \psi_{\mathfrak{b}}$ is valid. We abuse notation and write $\mathbb{b} \Leftarrow \mathfrak{b}^{\prime}$. Observe that this is equivalent to $\left[\left[\psi_{\mathfrak{b}}\right] \subseteq\left[\left[\psi_{\mathfrak{b}^{\prime}}\right]\right.\right.$. 
Constraints. A constraint over a set A of atoms is a triplet $\varphi=(\mathrm{q}, \mathbb{C}, \mathfrak{b})$ where state0f $(\varphi)=q \in \mathcal{Q}$ is the state of the constraint, $\operatorname{val}(\varphi)=\mathbb{C}$ is its minimal valuation, and $\operatorname{box}(\varphi)=\mathbb{b}$ over $A$ is its box. We use $\Phi$ to mean a set of constraints. A constraint $(\mathfrak{q}, \mathbb{C}, \mathbb{b})$ is well-formed if $\psi_{\mathfrak{b}}[\mathbb{C}]$ holds. We only consider well-formed constraints. We write $c l o(\mathbb{C}, \mathfrak{b})$ to mean the conjunct $\left(\wedge_{c t \in \mathcal{C}}(\mathbb{C}(c t) \unlhd c t) \wedge \psi_{b}\right)$. Intuitively, clo $(\mathbb{C}, \mathbb{b})$ denotes those valuations that are both "in the box" and in the $₫$-upward closure of $\mathbb{C}$. We let $[[(q, \mathbb{C}, \mathfrak{b})]]$ be the set $\left\{\left(\mathrm{q}, \mathbb{C}^{\prime}\right) \mid \mathbb{C}^{\prime} \in[[\mathrm{clo}(\mathbb{C}, \mathbb{b})]]\right\}$. This set contains at least $(\mathrm{q}, \mathbb{C})$ by wellformedness. Given two constraints $(\mathrm{q}, \mathbb{C}, \mathfrak{b})$ and $\left(\mathrm{q}^{\prime}, \mathbb{C}^{\prime}, \mathfrak{b}^{\prime}\right)$, we write $(\mathrm{q}, \mathbb{C}, \mathfrak{b}) \subseteq$ $\left(\mathrm{q}^{\prime}, \mathbb{C}^{\prime}, \mathfrak{b}^{\prime}\right.$ ) to mean that: (i) $\mathrm{q}=\mathrm{q}^{\prime}$, and (ii) $\mathbb{C} \unlhd \mathbb{C}^{\prime}$, and (iii) $\mathfrak{b} \Leftarrow \mathbb{b}^{\prime}$. Observe that $\varphi \sqsubseteq \varphi^{\prime}$ implies $\left[\left[\varphi^{\prime}\right]\right] \subseteq[[\varphi]]$. A subset $\Phi$ of a set of constraints $\Phi^{\prime}$ is minimal if: (i) $\varphi_{1} \nsubseteq \varphi_{2}$ for any pair of different constraints $\varphi_{1}, \varphi_{2} \in \Phi$, and (ii) for any $\varphi^{\prime} \in \Phi^{\prime}$, there is a $\varphi \in \Phi$ such that $\varphi \sqsubseteq \varphi^{\prime}$. We can show the following lemma.

Lemma 35. For a finite set of atoms A over $\mathcal{C}$, the ordering $ᄃ$ is a wellquasi-ordering $(\mathcal{W Q O})$ over the set of well-formed constraints over A. In addition, we can compute, for any set $\Phi$ of constraints, a finite ㄷ-minimal subset $\operatorname{Min}_{\sqsubseteq}(\Phi)$.

Image computations. Assume a conjunct $\psi$ over $\mathcal{C}$ and a guarded command $\mathrm{op}=\mathrm{grd} \Rightarrow \mathrm{cmd}$ for some $\mathrm{t} \in \mathrm{T}$. Recall that $\mathrm{Ax}$ is a set of auxiliary variables ranging over $\mathbb{N}$, grd is a predicate in preds $0 f_{\text {exprsof }(\mathcal{C} \cup A x)}$, and cmd is of the form $\mathrm{ct}_{1}, \ldots, \mathrm{ct}_{n}:=\mathrm{e}_{1}, \ldots, \mathrm{e}_{n}$ where, for each $i: 1 \leq i \leq n$,

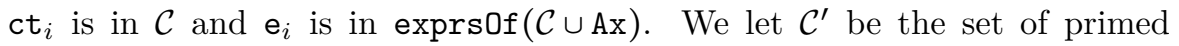
versions of all variables appearing in the left-hand side of $\mathrm{cmd}$. We write pre $_{\text {op }}(\psi)$ to mean a set of conjuncts whose disjunction is equivalent to $\left(\exists \mathcal{C}^{\prime} \cup \mathrm{Ax} .\left(\wedge_{1 \leq i \leq n}\left(\mathrm{ct}_{i}^{\prime}=\mathrm{e}_{i}\right) \wedge \operatorname{grd} \wedge \psi\left[\left\{\mathrm{ct} \leftarrow \mathrm{ct}^{\prime} \mid \mathrm{ct}^{\prime} \in \mathcal{C}^{\prime}\right\}\right]\right)\right)$. We also write post $_{\text {op }}(\psi)$ to mean a set of conjuncts whose disjunction is equivalent to $\left(\exists \mathcal{C} \cup \mathrm{Ax} .\left(\wedge_{1 \leq i \leq n}\left(\mathrm{ct}_{i}^{\prime}=\mathrm{e}_{i}\right) \wedge \operatorname{grd} \wedge \psi\right)\right)\left[\left\{\mathrm{ct}^{\prime} \leftarrow \mathrm{ct} \mid \mathrm{ct} \in \mathcal{C}\right\}\right]$. We naturally extend $\operatorname{pre}_{\mathrm{op}}(\psi)$ and post ${ }_{\mathrm{op}}(\psi)$ to sets of conjuncts.

Lemma 36. Assume an extended counter machine transiton $t \in \mathrm{T}$ and conjuncts $\Psi$. We can compute $\operatorname{pre}_{\mathrm{op}(\mathrm{t})}(\Psi)$ and post $_{\mathrm{op}(\mathrm{t})}(\Psi)$ such that $\left.\left[\operatorname{pre}_{\mathrm{op}(\mathrm{t})}(\Psi)\right]\right]$ equals $\left\{\mathbb{C} \mid(\operatorname{src}(\mathrm{t}), \mathbb{C}) \stackrel{\mathrm{t}}{\rightarrow}\left(\operatorname{dst}(\mathrm{t}), \mathbb{C}^{\prime}\right)\right.$ with $\mathbb{C}^{\prime} \in[[\Psi]\}$ and $\left[\operatorname{ppost}_{\mathrm{op}(\mathrm{t})}(\Psi)\right]$ equals $\left\{\mathbb{C}^{\prime} \mid(\operatorname{src}(\mathrm{t}), \mathbb{C}) \stackrel{\mathrm{t}}{\rightarrow}\left(\operatorname{dst}(\mathrm{t}), \mathbb{C}^{\prime}\right)\right.$ with $\left.\mathbb{C} \in[[\Psi]]\right\}$.

Grounded constraints and symbolic sets. A grounded constraint is a pair $\gamma=((\mathbf{q}, \mathbb{C}, \mathfrak{b}), \psi)$ that consists of a constraint constraintof $(\gamma)=$ $(\mathrm{q}, \mathbb{C}, \mathfrak{b})$ and a conjunct groundOf $(\gamma)=\psi$. It is well-formed if: $(\mathrm{q}, \mathbb{C}, \mathbb{b})$ is well-formed, $\psi \Rightarrow \operatorname{clo}(\mathbb{C}, \mathbb{b})$ is valid, and $\mathbb{C} \in[[\psi]$. We only manipulate well-formed grounded constraints. Intuitively, the ground $\psi$ in 

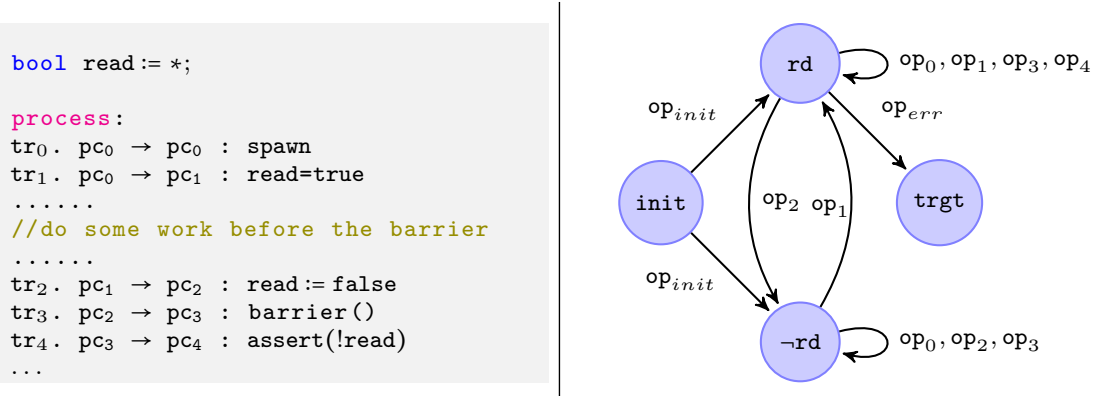

Figure 7.1: The counter machine to the right captures the behaviour of the concurrent program to the left. It makes use of one counter per program location. It involves the following guarded commands: $\mathrm{op}_{\text {init }}::=$ $\left(\mathrm{ct}_{0}, \mathrm{ct}_{1}, \mathrm{ct}_{2}, \mathrm{ct} \mathrm{t}_{3}, \mathrm{ct} \mathrm{t}_{4}:=1,0,0,0,0\right), \mathrm{op}_{0}::=\mathrm{ct}_{0} \geq 1 \Rightarrow \mathrm{ct}_{0}:=\mathrm{ct}_{0}+1$, $\mathrm{op}_{1}::=\mathrm{ct}_{0} \geq 1 \Rightarrow c t_{0}, c t_{1}:=c t_{0}-1, c t_{1}+1, \mathrm{op}_{2}::=c t_{1} \geq 1 \Rightarrow c t_{1}, \mathrm{ct}_{2}:=$ $c t_{1}-1, c t_{2}+1, o p_{3}::=\left(c t_{2} \geq 1 \wedge c t_{0}+c t_{1}=0\right) \Rightarrow\left(c t_{2}, c t_{3}:=c t_{2}-1, c t_{3}+1\right)$, $\mathrm{op}_{4}::=\mathrm{ct}_{3} \geq 1 \Rightarrow c t_{3}, c t_{4}:=\mathrm{ct}_{3}-1, \mathrm{ct}_{4}+1$, and op $\mathrm{prr}:\left(\mathrm{ct}_{3} \geq 1\right)$. The resulting system is not well-structured because of the test for zero in $\mathrm{op}_{3}$.

$((\mathrm{q}, \mathbb{C}, \mathfrak{b}), \psi)$ represents the "non-approximated" part of the $\unlhd$-upward closure of $\mathbb{C}$. This information will be needed for refining the preorder during the analysis. We abuse notation and write constraintOf $(\Gamma)$, resp. groundOf $(\Gamma)$, to mean the set of constraints, resp. grounds, of a set $\Gamma$ of grounded constraints. A trace $\rho$ of length $n$ is a sequence starting with a grounded constraint followed by $n$ transitions and grounded constraints. We say that two traces $\left(\varphi_{0}, \psi_{0}\right) ; \mathrm{t}_{1} ;\left(\varphi_{1}, \psi_{1}\right) ; \ldots ; \mathrm{t}_{n} ;\left(\varphi_{n}, \psi_{n}\right)$ and $\left(\varphi_{0}^{\prime}, \psi_{0}^{\prime}\right) ; \mathrm{t}_{1}^{\prime} ;\left(\varphi_{1}^{\prime}, \psi_{1}^{\prime}\right) ; \ldots ; \mathrm{t}_{n^{\prime}}^{\prime} ;\left(\varphi_{n^{\prime}}^{\prime}, \psi_{n^{\prime}}^{\prime}\right)$ are equivalent if: (i) $n=n^{\prime}$, and (ii) $\mathrm{t}_{i}$ is the same as $\mathrm{t}_{i}^{\prime}$ for each $i: 1 \leq i \leq n$, and (iii) $\varphi_{i} \sqsubseteq \varphi_{i}^{\prime}, \varphi_{i}^{\prime} \sqsubseteq \varphi_{i}$ and $\psi_{i} \Leftrightarrow \psi_{i}^{\prime}$ for each $i: 0 \leq i \leq n$. A symbolic set Sym is a set of pairs of grounded constraints and traces. Given a symbolic set Sym, we also use constraint0f(Sym) to mean all constraints $\varphi$ appearing in some $((\varphi, \psi), \rho)$ in Sym. Recall that we can compute a set $\operatorname{Min}_{\sqsubseteq}$ (constraintOf(Sym)) of ㄷ-minimal constraints for constraint0f(Sym).

\subsection{Illustrative Example}

We use the example depicted in Figure 7.1 to give an intuition of the lazy heuristics described in this chapter. A more detailed description follows in Section 7.5.

In the concurrent program to the left of Figure 7.1, a single process starts executing the program and it can spawn arbitrarily many concurrent processes using transition $\operatorname{tr}_{0}$. Assume all of the processes asynchronously run the same program. Each process can then set the shared flag read to true, and 
perform some reading followed by resetting read. All of the processes wait at the barrier. Observe that read should be false after the barrier since all processes that reached $\mathrm{pc}_{3}$ must have executed $t r_{2}$. The assertion at $\mathrm{pc}_{3}$ should therefore hold no matter how many processes are spawned. Capturing the barrier behaviour is crucial to establish that it is not possible to violate the assertion. The barrier behaviour while having the possibility that a single process changes location is inherently non-monotonic (adding more processes does not keep the barrier open). In Chapter 6, we introduced our approach that combines different abstraction techniques to automatically generate nonmonotonic counter machines such as the one to the right of Figure 7.1. For this case, the assertion in the concurrent program is violated iff the target state is reachable in the counter machine. We explain briefly in the following how such counter machines are generated.

Our tool, PACMAN (Chapter 6), takes as input concurrent programs similar to the one to left of Figure 7.1. It automatically performs predicate, counter and monotonic abstractions on them and generates counter machines that over-approximate the behaviour of the original program. It then tries to solve the reachability problem for those machines.

Given a concurrent program, PACMAN starts by generating concurrent Boolean programs by performing predicate abstraction and incrementally improving the abstraction in a CEGAR loop [50]. This results in a Boolean concurrent program that has the same control flow graph as the original program, but consists of only Boolean variables. To the obtained Boolean program, PACMAN applies counter abstraction to generate a counter machine. Intuitively, a counter is associated to each local state valuation of a process (which consists of the location and the valuation of the local variables of the process). Each state in the machine is also associated to a valuation of shared variables. An extra state is reserved for the target. The statements of the Boolean program are then translated as transitions of the counter machine.

For instance, in Figure 7.1, counters $\mathrm{ct}_{i}$, for $i: 0 \leq i \leq 4$, correspond respectively to the number of processes in program location $\mathrm{pc}_{\mathrm{i}}$ (the processes have no local variables here). Similarly, each transition $\mathrm{op}_{i}$ is associated with each $\operatorname{tr}_{i}$. There are two additional transitions $\mathrm{op}_{\text {init }}$ and $\mathrm{op}_{\text {err }}$ to model transitions involving initial or target states.

Note that the original concurrent program has non-monotonic invariants. For instance, transitions such as barriers, or any transition that tests variables representing the number of processes satisfying some property, do not necessarily stay enabled if we add more processes to the program. At the same time, the Boolean concurrent programs generated above are inherently monotonic. This corresponds to a loss of precision. Thus, proving correctness of those programs whose correctness depends on respecting the non-monotonic behaviour (e.g., the one enforced by a barrier) can become impossible. As a remedy to this fact, PACMAN automatically strengthens counter machine transitions by enforcing barrier invariants or by deriving new invariants (e.g., 
using an instrumented thread-modular analysis) to regain some of the precision. This helped us to verify several challenging benchmarks. For example, consider the transition $\mathrm{tr}_{3}$ in the program to the left of Figure 7.1. At the moment a process crosses the barrier and it is the first one to do so, there should be no process before location $\mathrm{pc}_{2}$. This fact holds afterwards and forbids any process from setting the flag read when some process is checking the assertion. The transition $\mathrm{op}_{3}$ is its corresponding transition in the strengthened counter machine. To ease the presentation of the example, $\mathrm{op}_{3}$ is strengthened with the guard $\left(c t_{0}+c t_{1}=0\right)$. (Observe that this is a simplification to ease the presentation; we can more faithfully capture the barrier by combining the test with a global flag.)

Plain monotonic abstraction [4] proceeds backwards while systematically closing upwards with respect to the ordering $\leq$ on $\mathrm{C}$ defined as follows. Recall that for configurations $c=(q, \mathbb{C})$ and $c^{\prime}=\left(q^{\prime}, \mathbb{c}^{\prime}\right), c \leq c^{\prime}$ iff $(1) q=q^{\prime}$, and $(2) \mathbb{C} \unlhd \mathbb{C}^{\prime}$. The trace depicted in Figure 7.2 is a generated false positive. In this description, for $i: 0 \leq i \leq 7$, we write $\gamma_{i}=\left(\varphi_{i}, \psi_{i}\right)$ to mean the grounded constraint with $\psi_{i}$ and the constraint $\varphi_{i}=\left(\mathrm{q}_{i}, \mathbb{C}_{i}, \mathfrak{b}_{i}\right)$. Intuitively, the grounded constraint represents "exact" valuations while the constraint captures over-approximations that are of the form $\left(q_{i}, \mathbb{C}\right)$ where $\mathbb{C}_{i} \unlhd \mathbb{C}$ and $\mathbb{C}$ satisfies $\psi_{\mathfrak{b}_{i}}$. The computation starts from the grounded constraint $\gamma_{7}=$ ( $\left(\right.$ trgt $\left.\left., \mathbb{C}_{7}, b_{\text {true }}\right), \psi_{7}\right)$ where $\psi_{7}$ is $\wedge_{\text {ct } \in \mathcal{C}}(\mathrm{ct} \geq 0)$ (always implicit). For $\gamma_{7}$, the exact and the over-approximated parts coincide.

The trace then computes $\psi_{6}=\left(\mathrm{ct}_{3} \geq 1\right)$ which captures the valuations of the predecessors of ( $\operatorname{trgt}, \mathbb{C}_{7}, \mathfrak{b}_{\text {true }}$ ) with respect to the transition ( $\left.r d, o p_{e r r}, \operatorname{trgt}\right)$. This set happens to be upward-closed and there is no need for approximation, hence $\gamma_{6}=\left(\left(r d, \mathbb{C}_{6}, \mathfrak{b}_{\text {true }}\right), \psi_{6}\right)$. Valuations of the exact predecessors of $\left(r d, \mathbb{C}_{6}, b_{\text {true }}\right)$ with respect to $\left(r d, o p_{3}, r d\right)$ are captured with the conjunct $\psi_{5}=\left(c t_{0}=c t_{1}=0 \wedge c t_{2} \geq 1\right)$. According to monotonic abstraction, these are approximated with the conjunct $\left(c t_{0} \geq 0 \wedge c t_{1} \geq 0 \wedge c t_{2} \geq 1\right)$. Continuing to compute the predecessors and closing upwards leads to the constraint $\varphi_{0}$ which involves the initial state init. The trace is reported as a possible reachability witness. It is well-known [4] that upward-closed sets are not preserved by non-monotonic transitions (such as those transitions involving $\mathrm{op}_{3}$ in Figure 7.1). At the same time, maintaining an exact analysis makes it impossible to guarantee termination.

Following the trace in the forward direction (from the left of Figure 7.1), it turns out that the upward closure that resulted in $\gamma_{5}$ is the one that made the spurious trace possible. Indeed, it is its approximation that allowed the counter $c t_{1}$ to be non-zero. This new value for $c t_{1}$ is the one that allowed the machine to execute $\left(\neg \mathrm{rd}, \mathrm{op}_{1}, \mathrm{rd}\right)$ backwards from $\varphi_{5}$, making it possible to reach the initial state. The constraint $\varphi_{5}$ is the pivot constraint of the trace. Constrained monotonic abstraction (CMA) proposes to refine the used ordering by strengthening it with a relevant predicate. In this case, ct $_{1} \leq$ 0 is used for strengthening, but in general (the atoms of) any predicate in 


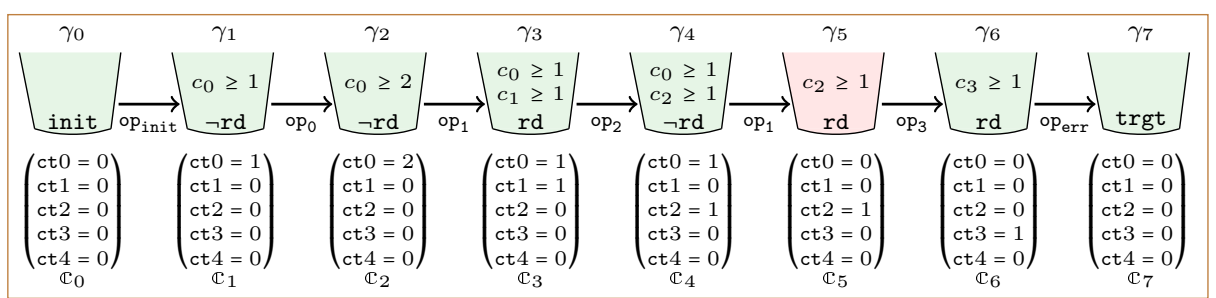

Figure 7.2: A spurious trace generated by monotonic abstraction. The $\gamma_{5}$ constraint introduces the first over-approximation that makes the spurious trace possible. The configuration $\left(r d, \mathbb{C}_{5}\right)$ is the pivot configuration of the spurious trace.

preds $0 f^{\operatorname{exprs} 0 f(\mathcal{C})}$ that separates the exact predecessors from the reachable part of the upward closure would do.

Different approaches can be taken regarding how to continue the exploration by using the constraint monotonic abstraction to remove the spurious counterexample. We briefly compare them in the following discussion of the example.

Eager CMA [3]. The exploration is restarted from scratch and $\left(\mathrm{ct}_{1} \leq 0\right)$ is used to systematically partition all exact predecessors. The upward closure is constrained to not alter the refinement predicate. Therefore, all generated valuations are approximated with the stronger ordering. Localizing refinement can make possible both the reuse of a potentially large part of the explored state space and application of the (slower) refinement to a smaller number of sets.

Lazy CMA. When backtracking, we only eliminate those constraints that were obtained as descendants of a constraint that caused the spurious trace and therefore needs to be refined. We refer to this constraint as the pivot constraint, and to its minimal configuration as the pivot configuration. In fact, we identify three localization heuristics:

- point-based-lazy. We associate the refinement predicates with the pivot configurations. Later in the exploration, when we hit a new pivot configuration, we constrain with respect to those predicates that were already associated with it. 
- order-based-lazy. The point-based approach may be too localized as there is an infinite number of pivot configurations. For instance, a similar trace can continue, after $\left(r d, c t_{2}=1\right)$, with $\mathrm{op}_{1}$ and get to the minimal configuration sending $\mathrm{ct}_{2}$ to 2 . This one is different from the mapped pivot configuration, and hence, we need to introduce a new pivot configuration with the same predicate $c_{0} \leq 0$. This approach considers the predicates of all larger or smaller pivot configurations. The idea is that if the predicate was important for the mapped pivot configuration, then it must have been to separate it from a reachable upward closed part, and hence, it may be relevant.

- descendants-based-lazy. In addition to associating refinement predicates with pivot configurations as in the point-based approach, this heuristic leverages the fact that predicates may remain relevant for a sequence of transitions. Here we compare the exact predecessors with the predicates used to constrain the upward closure of the parent. If those predicates still hold for the predecessors, then we maintain them when closing upwards. This heuristic bears similarity to the forward propagation of clauses in IC3 [85]. In the IC3 algorithm the clauses are propagated in the trace from a preceding formula to the succeeding one if they still hold.

\subsection{State Reachability Checking}

In this section, we describe four different forward CMA variants (eager, pointbased-lazy, order-based-lazy and descendants-based-lazy). The four procedures can also be applied backwards (as described in the experiments of Section 7.6). The four variants use grounded constraints as symbolic representations for a possibly infinite number of machine configurations. The symbolic representation is refined using atoms that are obtained using a counterexample-guided refinement scheme. The difference between the four variants lies in the way the discovered predicates (in fact, atoms for simplifying the presentation) are associated with the new symbolic representations and in the way backtracking is carried out. We start by introducing the basic "partition" procedure.

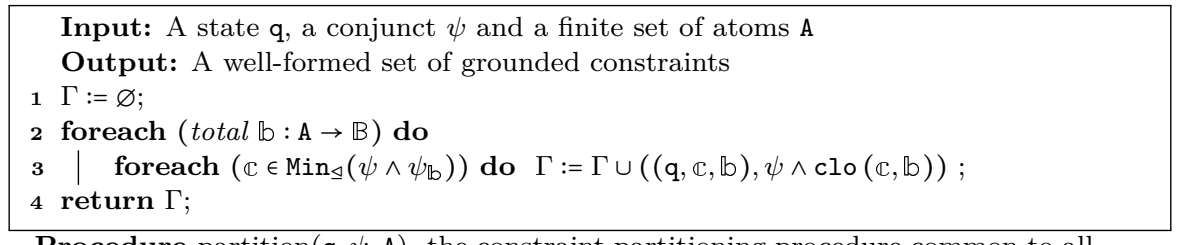

Procedure partition(q, $\psi, \mathrm{A})$, the constraint partitioning procedure common to all variants.

Partition. "partition(q, $\psi, \mathrm{A})$ " partitions $\psi$ according to all atoms in A. Each obtained conjunct is further decomposed according to its $\unlhd$-minimal val- 
uations. Conjuncts are then used to build a well-formed grounded constraint $\left((\mathbf{q}, \mathbb{C}, \mathbb{b}), \psi^{\prime}\right)$ where $\mathbb{b}$ is a box over $\mathrm{A}$. Observe that the disjunction of the grounds of the obtained grounded constraints is equivalent to $\psi$. Soundness is stated in Lemma 37.

Lemma 37. Assume a finite set A of atoms. For any conjunct $\psi$, it is the case that $[[(\mathrm{q}, \psi)]]=\left\{(\mathrm{q}, \mathbb{C}) \mid \mathbb{C} \in\left[\left[\psi^{\prime}\right]\right]_{\geq 0}\right.$ for each $\left.\psi^{\prime} \in \operatorname{groundOf}(\operatorname{partition}(\mathrm{q}, \psi, \mathrm{A}))\right\} \subseteq$ [ constraintOf $(\operatorname{partition}(\mathrm{q}, \psi, \mathrm{A}))]$.

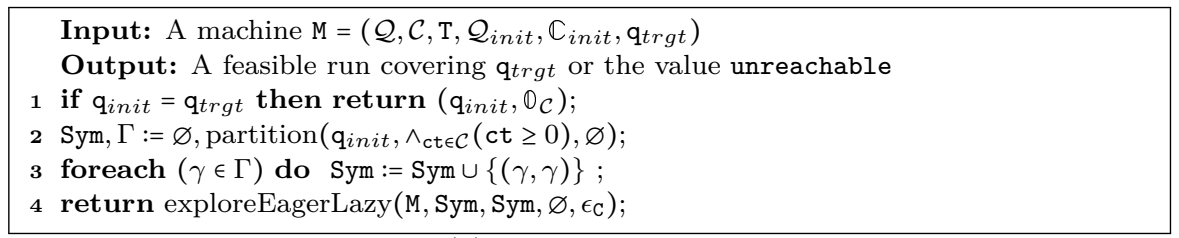

Procedure checkReachability $(\mathrm{M})$, the common entry point for all variants.

Eager CMA, like the other variants, starts by passing a description of the machine to the "checkReachability" procedure. It either returns a feasible run covering $\mathrm{q}_{\text {trgt }}$, or states that there are no such runs. The procedure returns directly (line 1) if initial and target states coincide. It then calls "partition" to obtain a set of well-formed grounded constraints that together capture all initial configurations. These are passed to the "exploreEagerLazy" procedure.

Explore. "exploreEagerLazy $(M$, working, store, sleep, $\mathbb{E})$ " results in a working list procedure that maintains three symbolic sets working, store and sleep. sleep is only relevant for the lazy variants. The partial mapping $\mathbb{f}: \mathrm{C} \rightarrow$ atoms $0 f(\mathcal{C})$ encapsulates all refinement predicates discovered so far and is therefore empty when the procedure is called from "checkReachability". Intuitively, $\mathbb{E}(\mathrm{c})$ associates with the pivot configuration $\mathrm{c}$ those predicates that helped eliminate a false positive when $\mathrm{c}$ was the minimal configuration of the constraint that made the false positive possible. We will explain how $\mathbb{E}$ is updated when introducing the procedure "simulate". The symbolic set working is used for the grounded constraints that are yet to be visited (i.e., for which the successors are still to be computed and approximated). The store set is used for both those grounded constraints that have been visited and for those in working. The sleep set corresponds to those constraints that might have to be visited but for which there is an ᄃ-equivalent representative in store. In case a backtracking eliminates the representative in store, the corresponding grounded constraint in sleep has to be reconsidered. This is explained in the "backtrack" procedure of the lazy variants.

The procedure picks a pair $((\varphi, \psi), \rho)$ from working where $\varphi$ is a member of $\operatorname{Min}_{\sqsubseteq}$ (constraintOf(store)). If the initial state is reached, it calls procedure "simulate" to check the associated trace and to backtrack if needed 
(lines 4-5). Otherwise, it iterates through all transitions $\mathrm{t}$ in $\mathrm{T}$ and computes an exact representation of the predecessors of the constraint. The call "decompose (q, $\left.\psi_{p}, \mathbb{f}, \mathfrak{b}\right)$ " boils down, for the eager variant, to a call to "parti$\operatorname{tion}\left(\mathrm{q}, \psi_{p}\right.$, image $\left.(\mathbb{F})\right)$ ". The obtained grounded constraints are used in order to update the working, store, and sleep symbolic sets.

If no pair was picked at line 1, then the exploration is finished and the procedure returns unreachable. In fact, pairs are never removed from store if no target states are encountered at line 4 . In addition, two pairs with ㄷequivalent constraints cannot be added to working (lines 10-13). For this reason, executing the first line for an infinite number of times without calling the procedure "simulate" would result in an infinite sequence of constraints that would violate Lemma 35 .

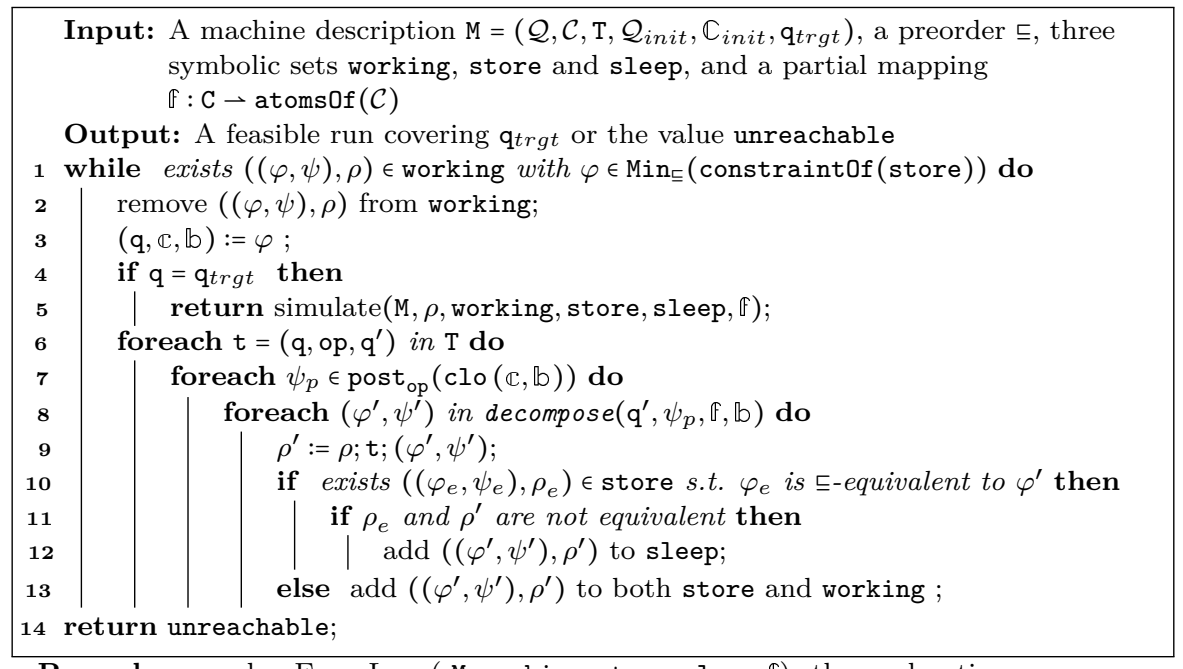

Procedure exploreEagerLazy( $M$, working, store, sleep, $\mathbb{f}$ ), the exploration procedure common to all variants.

Simulate. This procedure checks feasibility of a trace $\rho$ from $\mathrm{q}_{\text {init }}$ to $\mathrm{q}_{\text {trgt }}$. The procedure incrementally builds a sequence of sets of conjuncts $\Psi_{n}, \ldots, \Psi_{0}$ where each $\Psi_{i}$ intuitively denotes the valuations that are backwards reachable from $\mathrm{q}_{\text {trgt }}$ after $k$ steps of $\rho$ (starting from $k=0$ ), and are still denoted by clo $\left(\mathbb{C}_{(n-k)}, \mathbb{b}_{(n-k)}\right)$. The idea is to systematically intersect (a representation of) the successors of step $k$ with the grounded constraint that gave rise to the constraint at step $k+1$. If the procedure finds a satisfiable $\Psi_{0}$, then a run can be generated by construction. Such a run is then returned at line 8 . Otherwise, there must have been a step where the "exact" set of conjuncts does not intersect the conjunct representing the exact part that gave rise to the corresponding constraint. In other words, the trace could be eliminated by strengthening the over-approximation at line 7 of the "exploreEagerLazy" procedure. In this case, (at line 6 of the "simulate" procedure), new refinement atoms are identified using an off-the-shelf interpolation procedure for 
QF_LIA (Quantifier-Free Linear Integer Arithmetic). This information will be used differently by the eager and lazy variants when calling their respective "backtrack" procedures.

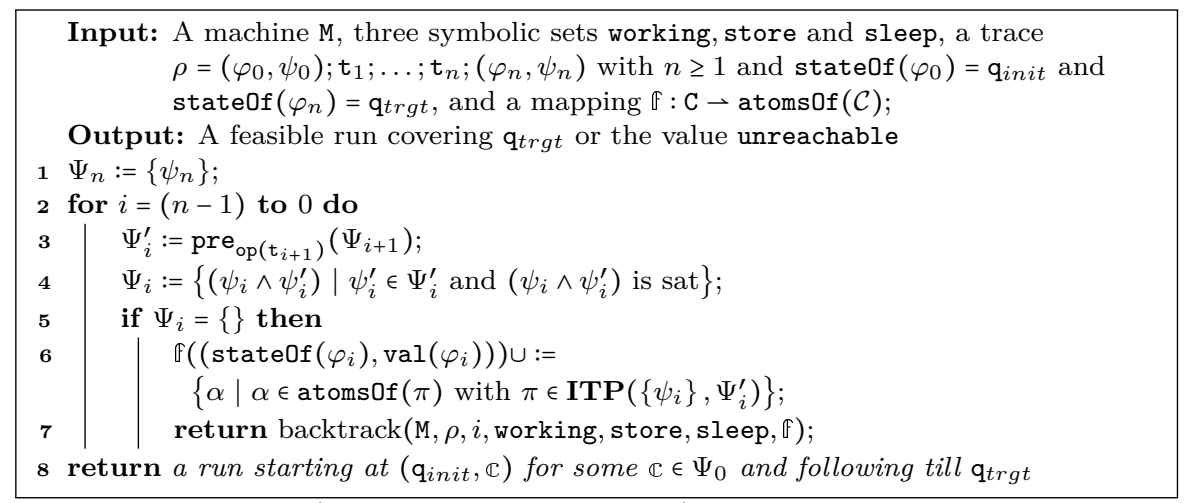

Procedure simulate( $\mathrm{M}, \rho$, working, store, sleep, $\mathbb{E}$ ), the simulation procedure common to all variants.

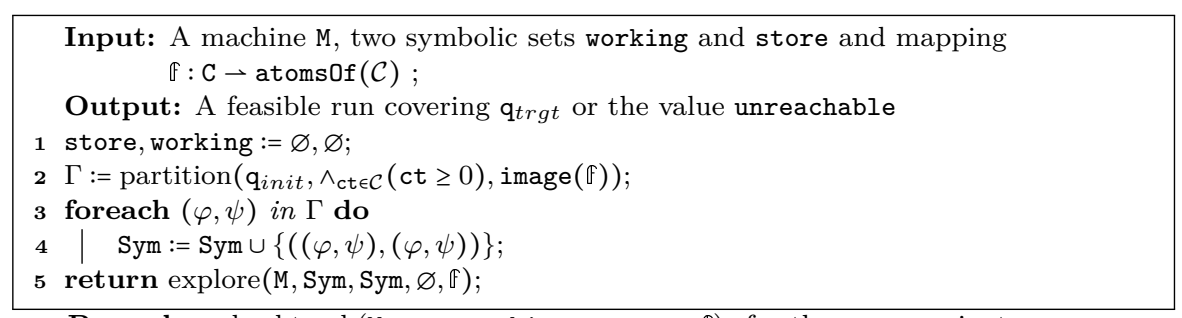

Procedure backtrack $(\mathrm{M}, \ldots, \ldots$, working, store, _, $\mathbb{f})$, for the eager variant.

Eager backtracking throws away the explored state space (line 1) and restarts the computation from scratch using the new refinement atoms captured in $\mathbb{E}$.

Lazy backtracking. Intuitively, all three lazy approaches reuse the part of the explored state space that is not affected by the new refinements. This is done by restarting the exploration from new sets working and store that are obtained after pruning away the pivot constraint identified by the argument $i$ passed by "simulate" together with all its descendants (identified in lines 1-6). One important aspect is that the grounded constraints that have not been added to store at line 11 of the "exploreEagerLazy" procedure may have been discarded for the wrong reason (i.e., there was an ㄷ-equivalent constraint that needs to be pruned away now). This would jeopardize soundness. For this reason, we maintain the sleep set for tracking the discarded grounded constraints that have to be put back into working and store if the constraint that blocked them is pruned away (see lines 4-6). The refined pivot is added to the new sets working and store (lines 10-13). Lines 7-9 are only used 
by the descendants-based approach which takes into account the box of the parent.

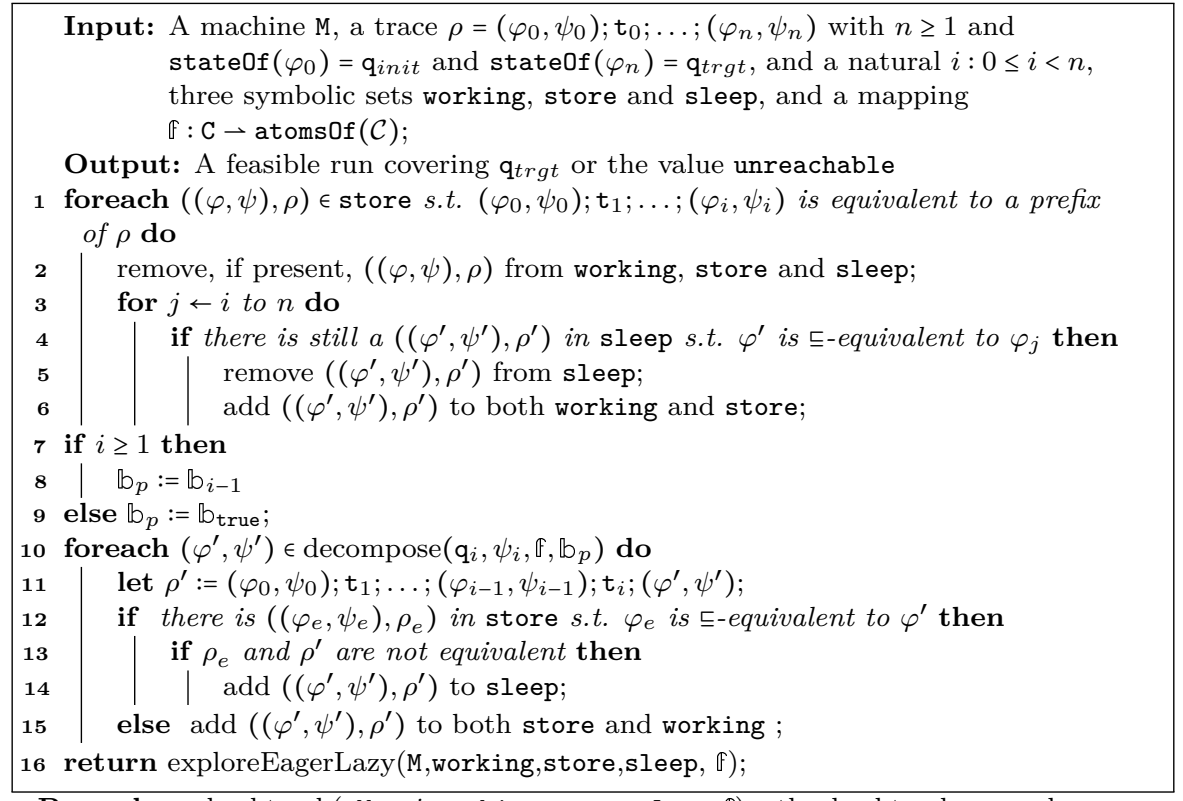

Procedure backtrack( $\mathrm{M}, \rho, i$, working, store, sleep, $\mathbb{E})$, the backtrack procedure common to all lazy variants.

The main difference between the lazy variants is in the way their respective "decompose" procedures associate refinement atoms with "exact" conjuncts.

Point-based. This variant is the one that "localizes" the refinement to the greatest degree. Each time an obtained grounded conjunct is considered for approximation, it checks whether its minimal valuation has already been associated with some refinement atoms. If this is the case, it passes them when calling the "partition" procedure.

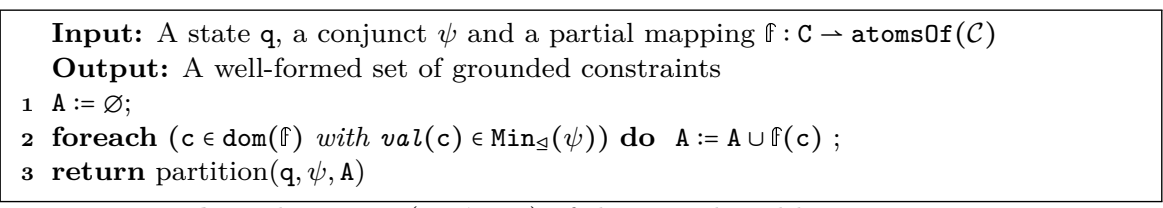

Procedure decompose( $\mathbf{q}, \psi, \mathfrak{f},-)$ of the point-based-lazy variant.

Order-based. This variant "localizes" less than the point-based variant. Each time an obtained "exact" conjunct is considered for approximation, it checks whether its minimal valuation is $\unlhd$-related to an already mapped val- 
uation. The union of all corresponding atoms is passed to the "partition" procedure.

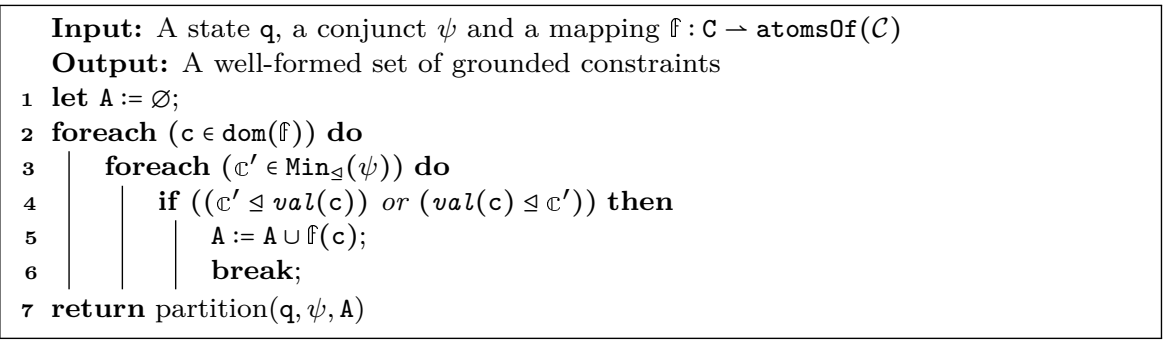

Procedure decompose $(\mathrm{q}, \psi, \mathbb{F}, \ldots)$ of the order-based variant.

Descendants-based. This variant "localizes" less than the point-based variant, but is incomparable with the order-based one. The idea is to keep those refinement atoms that were used for the parent constraint, and that are still weaker than the current conjunct that is to be approximated.

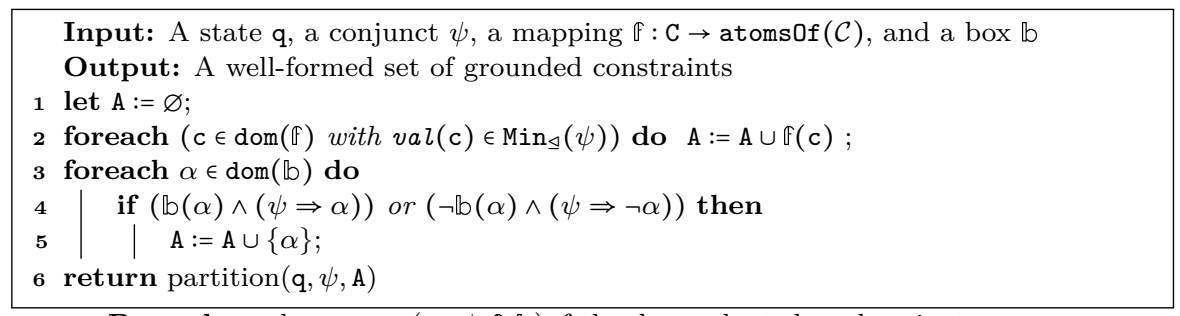

Procedure decompose $(\mathrm{q}, \psi, \mathfrak{F}, \mathfrak{b})$ of the descendants-based variant.

Finally, we state the soundness of our four exploration variants. The proof is by observing that store always represents, at the $i^{\text {th }}$ iteration of the loop of procedure "exploreEagerLazy", an over-approximation of the machine configurations obtained after $i$ steps, combined with Lemma 35 and Lemma 36 and by well-quasi-ordering of $\sqsubset$ on the set of constraints for a finite number of refinement atoms.

Theorem 22. All four exploration variants are sound. In addition, each call to the procedure "checkReachability" eventually terminates if only a finite number of calls to the procedure "simulate" are executed.

Proof. Let working $k$, store ${ }_{k}$ and sleep $_{k}$ be the sets working, store and sleep obtained at line 1 at the $k^{t h}$ iteration of the loop in procedure "exploreEagerLazy". We can show the following propositions:

(a) for every valuation $\mathbb{C},\left(\mathrm{q}_{\text {init }}, \mathbb{C}\right)$ belongs to $\left[\left[\right.\right.$ store $\left.\left._{k}\right]\right]$,

(b) every element $((\varphi, \psi), \rho)$ such that $\operatorname{stateOf}(\varphi)=q_{\text {trgt }}$ is in store $_{k}$ iff it is in working $k$,

(c) for each element $((\varphi, \psi), \rho)$ of store $_{k}$ such that $((\varphi, \psi), \rho) \notin$ working $_{k}$ and $\varphi \in \operatorname{Min}_{\sqsubseteq}\left(\right.$ constraintOf $\left(\right.$ store $\left.\left._{k}\right)\right)$ and for 
each transition $\mathrm{t}=\left(\mathrm{q}, \mathrm{op}, \mathrm{q}^{\prime}\right) \in \mathrm{T}$, the configurations in $\left\{\left(\mathrm{q}^{\prime}, \mathbb{C}^{\prime}\right) \mid \mathbb{C}^{\prime} \in\left[\left[\right.\right.\right.$ post $\left.\left.\left._{\text {op }}(\operatorname{clo}(\operatorname{val}(\varphi), \operatorname{box}(\varphi)))\right]\right]\right\}$ are also in $\left[\right.$ store $\left._{k}\right]$

For proposition (a), note that the elements covering all initial configurations will be added in the beginning to both working and store (lines 2-4 of "checkReachability"). Let an initial element be such an element. In case an initial element $((\varphi, \psi), \rho)$ is removed by "backtrack" (line 1 of the eager variant and line 2 of the lazy variant) which is the only place where elements may be removed from store, it will be replaced (in line 4 of the eager "backtrack" and line 6 of the lazy "backtrack") by a set of elements obtained from "decompose" whose union still denotes $\psi$ (Lemma 37).

For proposition (b) observe that whenever an element with the state $\mathrm{q}_{\text {trgt }}$ is added to store, it is also added to working (e.g., Line 13 of "exploreEagerLazy") and whenever such element is removed from store, it is also removed from working (Line 6 of "backtrack").

The intuition of proposition (c) is as follows. The algorithm always picks an element $((\varphi, \psi), \rho) \in$ working $_{k}$ such that $\varphi \in$ $\operatorname{Min}_{\sqsubseteq}\left(\right.$ constraintOf $\left(\right.$ store $\left.\left._{k}\right)\right)$ and computes its successors. Therefore, for any element $\left(\left(\varphi^{\prime}, \psi^{\prime}\right), \rho^{\prime}\right) \notin$ working $_{k}$ such that $\varphi^{\prime} \in$ $\operatorname{Min}_{\sqsubseteq}\left(\right.$ constraintOf $\left(\right.$ store $\left.\left._{k}\right)\right)$, the successors must be covered by elements in store $_{k}$ unless "simulate" and "backtrack" are called. In case "backtrack" is not called, the only places where store $_{k}$ and working $_{k}$ are manipulated are at lines 2 and 13 of "exploreEagerLazy". At line 2, an element $((\varphi, \psi), \rho) \in$ working $_{k}$ such that $\varphi \epsilon$ $\operatorname{Min}_{\sqsubseteq}$ (constraint0f $\left(\right.$ store $\left.\left._{k}\right)\right)$ is removed from working. At line 11, for each transition $\left(\operatorname{stateOf}(\varphi)\right.$, op, stateOf $\left.\left(\varphi^{\prime}\right)\right) \in \mathrm{T}, \operatorname{post}_{\text {op }}(\operatorname{clo}(\operatorname{val}(\varphi), \operatorname{box}(\varphi)))$ is computed, then all elements $\left(\left(\varphi^{\prime}, \psi^{\prime}\right), \rho, \mathrm{t},\left(\varphi^{\prime}, \psi^{\prime}\right)\right)$ such that the union of the grounded constraints $\left(\varphi^{\prime}, \psi^{\prime}\right)$ covers post op $(\operatorname{clo}(\operatorname{val}(\varphi), \operatorname{box}(\varphi)))$ are added to working and $_{\text {store }}$, in case another element with constraint $\varphi^{\prime}$

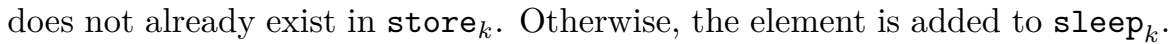
Thus, in case there is no call to "backtrack", the successors of any element that is removed from working $k$ are denoted by the elements in store . $_{\text {. }}$

We show that proposition (c) holds also when "backtrack" is called. Given a trace $\rho=\left(\varphi_{0}, \psi_{0}\right) ; \mathrm{t}_{1} ; \ldots ; \mathrm{t}_{n} ;\left(\varphi_{n}, \psi_{n}\right)$ and $0 \leq i<n$ as the index of the element to be refined, "backtrack" removes any element $((\varphi, \psi), \rho)$ whose trace

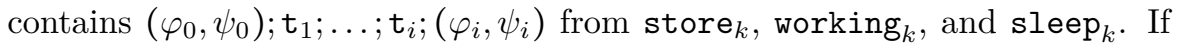
some of the elements removed from store $_{k}$ had 드-equivalent representative in $\mathrm{sleep}_{k}$, the representatives will be removed from $\mathrm{sleep}_{k}$ and added to store $_{k}$ and working $k$. This is done at lines 4-6 of "backtrack". As a result, proposition (c) holds.

Soundness. Suppose the algorithm returns unreachable. Then, at some iteration, there is no element $((\varphi, \psi), \rho)$ in working such that $\varphi \epsilon$ $\operatorname{Min}_{\sqsubseteq}($ constraintOf(store)). Combined with the propositions (a) and (c) of 
Theorem 22, we have that [ [store]] is a fixpoint that is an over-approximation of all reachable configurations. Proposition (b) ensures that no element with the state $\mathrm{q}_{\text {trgt }}$ exists in store when the algorithm returns unreachable.

Termination. The procedure "checkReachability" terminates if only a finite number of calls to procedure "simulate" are executed. This relies on the fact that when the number of calls to procedure "simulate" are finite, the only source of non-termination can be the while loop in "exploreEagerLazy" if the set constraintOf(working) $\cap \operatorname{Min}_{\sqsubseteq}$ (constraintOf(store)) never becomes empty. Suppose after finishing all the calls to "simulate" an infinite sequence of constraints $A=\varphi_{0}, \varphi_{1}, \ldots$ is obtained at Line 1 of the while loop while $i \geq 0$ is the iteration at which $\varphi_{i}$ was removed from working. Observe that for only a finite number of constraints in the sequence where $0 \leq i<j$ we have $\varphi_{i}$ is equivalent to $\varphi_{j}$ with respect to ㄷ. This is because Lines 6 and 15 of "backtrack" are the only lines in which elements are added to store and working as a result of a call to the procedure "simulate". In both cases, the constraint $\varphi^{\prime}$ of an element $\left(\left(\varphi^{\prime}, \psi^{\prime}\right), \rho^{\prime}\right)$ that is added to store and working might be ㄷ-equivalent to the constraints of the elements already in those sets. However, we are sure that the procedure "backtrack" is called finitely many times because it is only called once from the procedure "simulate". Hence, there exist only finitely many constraints in the sequence $A$ that have ㄷequivalent constraints. Assume we extract a sequence $B$ from the sequence $A$ such that $B$ contains only one representative for any set of ᄃ-equivalent constraints. Observe that in sequence $B$ for any $0 \leq i<j, \varphi_{i} \notin \varphi_{j}$. This is because 1) an element is removed from working only if is minimal in store with respect to $ᄃ$, and 2) elements are not removed from store when there is no call to the procedure "simulate". This sequence violates $\mathcal{W} \mathcal{Q O}$ of $ᄃ$.

\subsection{Experimental Results}

We have implemented our techniques in our open-source prototype tool ZAAmA. The tool and benchmarks are available ${ }^{1}$. The tool relies on the Z3 SMT solver [104] for its internal representations and operations.

The inputs of the prototype tool are counter machine encodings of Boolean concurrent programs with broadcasts and arbitrary tests (as described in Section 7.4). We have experimented with over eighty different counter machine reachability problems. These were obtained from our prototype tool PACMAN [61], which checks local (i.e., assertion) or global (e.g., deadlock freedom) properties in concurrent programs (some inspired by [52] and [40]).

Given a property to check in a concurrent program, PACMAN proceeds in predicate abstraction iterations. For each set of tracked predicates, it creates a counter machine reachability problem. Combining PACMAN with ZAAMA

\footnotetext{
${ }^{1}$ https://gitlab.liu.se/live/zaama.
} 


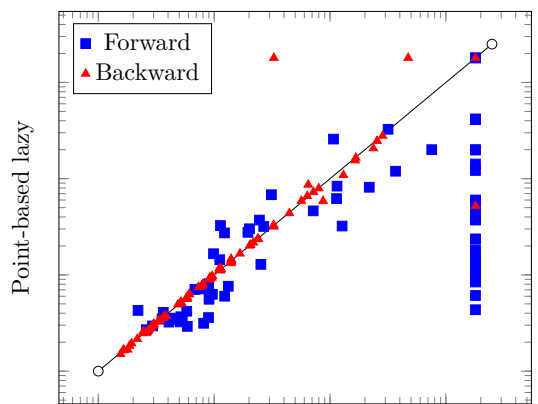

Order-based lazy

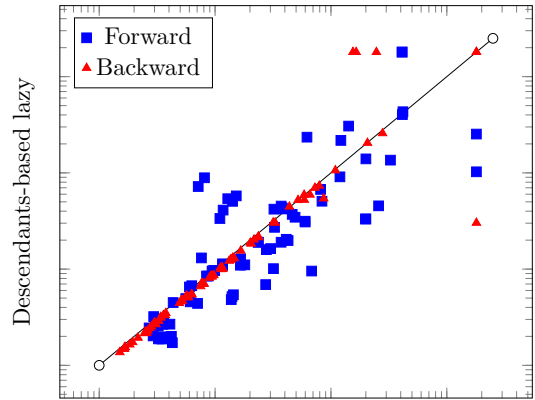

Point-based lazy

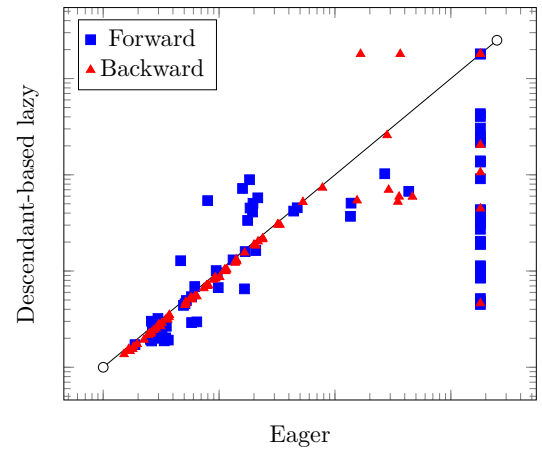

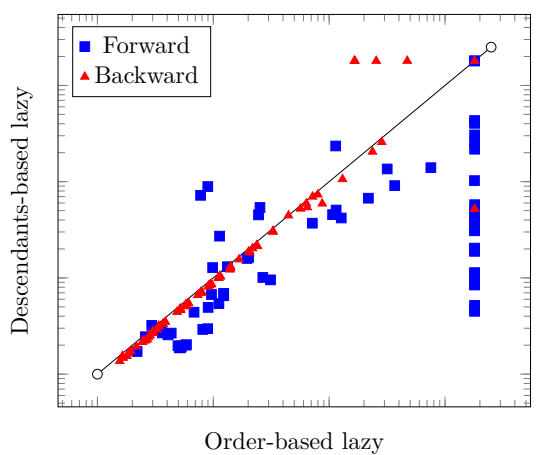
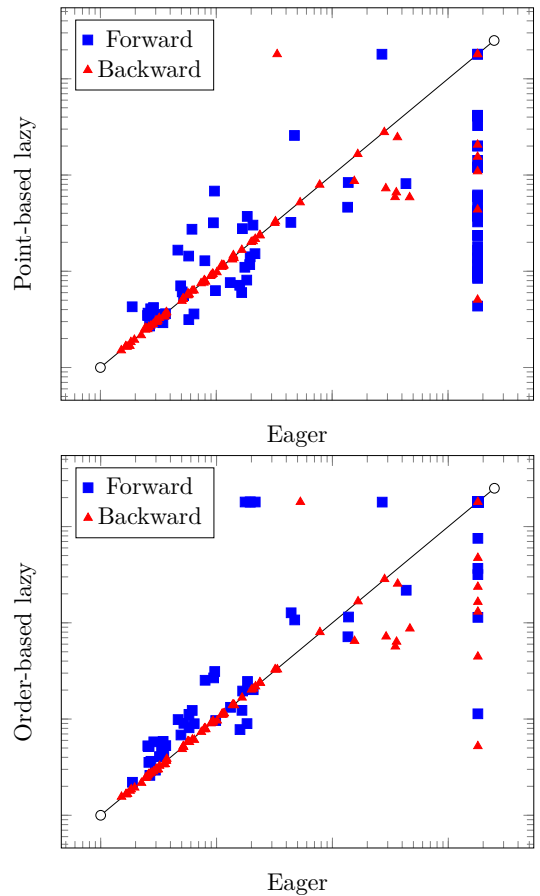

Figure 7.3: Comparing execution times of eager and lazy variants on a logarithmic scale. Each axis corresponds to a variant of monotonic abstraction and the execution times of the different variants are compared against each other.

results in a nested CEGAR loop: an outer loop for generating counter machine reachability problems, and an inner loop for checking the resulting problems. About $45 \%$ of the generated counter machines are not monotonic. We tested all of those machines separately with ZAAMA in different settings for each benchmark and reported the execution times. Thus, the PACMAN overhead is not included in the reported times. Note that although $55 \%$ of the examples are monotonic, they still need refinement in forward exploration. 
We also tested our benchmarks with the tool BREACH introduced in [82]. BREACH cannot take non-monotonic inputs and is inherently incapable of solving reachability problems for systems that are the main target of this chapter. Thus, we could apply it only to the monotonic benchmarks; for which, the runtime of BREACH was less than 5 seconds in each case. We consider this to be an encouraging result for adapting BREACH to non-monotonic systems. The challenge, however, is to have an under-approximation search engine for such systems and one could investigate the possibilities of developing such an engine or using acceleration tools such as FASTer [17].

We have chosen a time-out of 30 minutes for each of the variants: eager, point-based, order-based and descendants-based, both in forward and in backward exploration. We have conducted our experiments on an Intel Core i7 processor running at $2.93 \mathrm{GHz}$ with $8 \mathrm{~GB}$ of memory. We report on our results in Figure 7.3 where we consider, for each setting, all possible lazy pairs. Moreover, we consider the pairs that consist of the eager approach and a lazy approach.

The forward explorations turned out to be faster than the corresponding backward ones in about $25 \%$ of the examples. We expected the forward exploration to be slower as it needs several refinement steps because it starts from the initial configurations which are typically much more constrained than the target configurations. We considered the forward exploration because it offers more opportunities to test the effect of localizing the refinement in problems that typically require more refinement steps in forward. Indeed, the figures show that the analysis times of the different variants coincide more often when checking reachability backward than checking it forward, and overall, there have been many more time-outs in forward than in backward.

Furthermore, the lazy variants were able to conclude on most of the reachability problems, in fact each of the reachability problems has been solved by at least one of the lazy variants (except for one problem in the backward exploration), when the eager variant timed out on several of them. This is an encouraging result that confirms the advantages of localizing refinement. There are some cases where the eager variant did better than all lazy ones. These correspond to cases where localization required more refinement efforts to reach a conclusion.

We also observe that the order-based approach times out in about half of the forward searches, while the point-based only times out in two cases. This goes against the initial intuition that larger valuations would profit from the refinement predicates of the smaller ones. One explanation could be that if the larger valuation were to require the same predicate as the smaller one, then adding the predicate would result in a redundant representation that should be eliminated. It therefore seems that it does not take long for the point-based to discover this redundancy while still profiting from the localization of the refinement. Instead, the order-based uses predicates even when they are not 
proven to be needed resulting in finer grained symbolic elements that slow down the exploration.

It is interesting to observe that the descendants-based approach did better in the forward exploration than the point-based approach. One explanation could be that, in forward, relevant refinement interpolants sometimes correspond to weak inductive invariants that get propagated by this approach. When exploring backwards it seems that, at least for our examples the invariants corresponding to the "bad" configurations do not profit from this parent-child transmission.

\subsection{Conclusion}

We have introduced and discussed three different ways of localizing constrained monotonic abstraction in systems with infinite control. For this, we have targeted reachability problems for (possibly non-well-structured) counter machines obtained as abstractions of concurrent programs. Our new techniques allow us to systematically avoid trashing the explored state-space before encountering the false positives that necessitate the introduction of new refinement predicates. This allowed us to consistently improve on the existing eager exploration, both in forward and in backward explorations. Possible future works could consider combining forward and backward approximations, using the pivot configuration to make it possible to choose interpolants that are easier to generalize and assessing the feasibility of combination with new partial order techniques. 


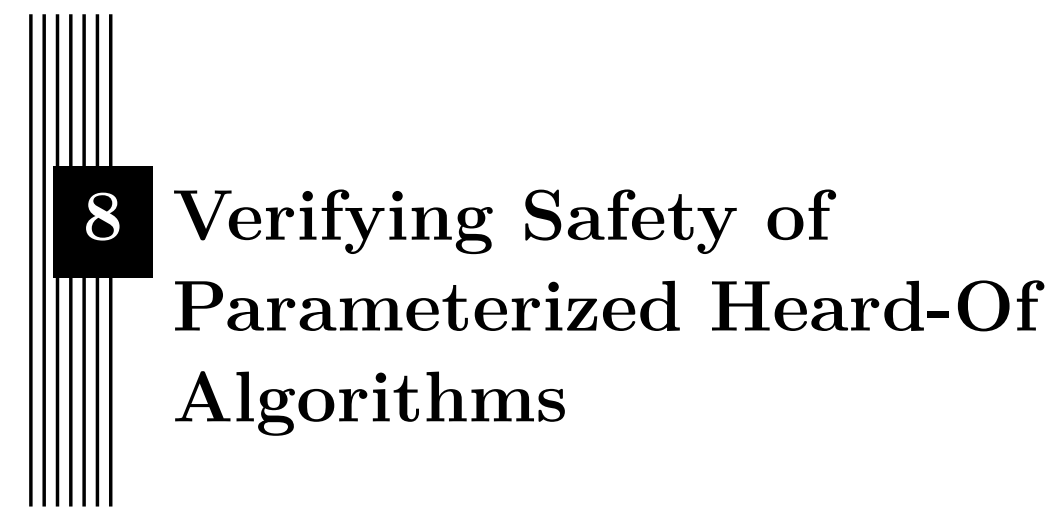

We consider the problem of automatically checking safety properties of faulttolerant distributed algorithms. We express the considered class of distributed algorithms in terms of the Heard-Of model, where arbitrarily many processes proceed in infinite rounds in the presence of failures such as message losses or message corruptions. For the considered class, we propose a sound but (in general) incomplete procedure that is guaranteed to terminate even in the presence of unbounded numbers of processes. In addition, we report on our experiments for which our approach either proved correctness or automatically found a concrete trace that violated the considered safety property.

\subsection{Introduction}

In general, it is difficult to prove the correctness of fault-tolerant distributed protocols. Such protocols are meant to operate in the presence of faults ranging from process crashes to message losses or corruption. We consider the parameterized case where arbitrarily many identical processes participate in running the distributed protocol. We adopt the popular Heard-Of model [30, 23]. This model uniformly describes distributed protocols in the presence of transmission-based failures, whether static or dynamic, permanent or transient. Protocols in the Heard-Of model proceed in rounds. Each process sends a message to other processes at each round, hears from some of them, and updates its state based on the received messages. Hence, at each round, a process "hears" from a set of other processes. Fault descriptions are captured 
by stating constraints on the possible sets of processes and the messages each process hears from others (e.g., each process hears from at least half of the processes or at most a third of the sent messages have been corrupted).

We consider the problem of automatically establishing the correctness of safety properties for parameterized distributed protocols expressed in the Heard-Of model. The safety properties we consider concern checking state reachability, i.e., reachability of configurations where a given number of processes are in some forbidden combination of states. Observe that we do not check whether the protocols have made progress. This would require us to account for communication predicates that ensure that the processes eventually hear from enough other processes. However, we need to constrain, depending on the environment we want to capture, that messages may be lost (benign crashes or transmission failures) or altered (corruption failure). For consensus protocols, this is enough to capture all executions that violate the agreement (two processes decide on different values), validity (a value is decided although no process proposed it) or irrevocability (a decided value is revoked). The verification problem is made difficult by the parameterization in the number of processes and by the allowed faults. Parameterization requires us to verify infinite families of the protocols, one for each number of participating processes. The transmission model allows each process to receive a subset of the sent messages (benign failures) in addition to some altered messages (corrupted communication), making this information local to the processes.

Related work. Abstractions for threshold-based fault-tolerant distributed systems were introduced in $[79,86]$. The work is extended to synchronous round-based semantics in [121]. These works can handle interesting faulttolerant protocols in the presence of different faults, such as Byzantine faults. However, they require the user to encode the distributed system in terms of a threshold automaton and propose interval-based over-approximations or bounded-model-checking-based under-approximations for the parameterized verification problem. The models we consider directly target protocols expressed in the Heard-Of model with a sound over-approximation and can account for message losses (omission fault) and message alteration (corruption fault). The work in [99] has the merit of proposing cutoffs for a syntactically restricted class of consensus protocols. The class is also expressed in the Heard-Of model. While we do not provide such cutoffs, our work can afford to check correctness for richer fragments that can more faithfully capture constructs such as "the number of received messages with value $v_{0}$ is at least two thirds the number of processes" as opposed to "the number of received messages is at least two thirds the number of processes and all of them have value $v_{0}$ ". The approach in [99] can verify Heard-Of protocols such as Paxos that we cannot verify in our current approach. Because in our current setting, 
we have only many-to-many transmissions, while we need to account for oneto-many and many-to-one transmissions to capture those protocols. However, they only consider benign faults for the protocols, but our approach can handle both benign and corruption faults. To the best of our knowledge, we are the first to verify Heard-of protocols in the presence of corruption faults.

Ongoing works [90, 9] study automatizing the process of deciding satisfiability of constraints that involve arbitrarily many processes and cardinality constraints over sets of received messages with specific properties. Such constraints naturally arise when verifying fault-tolerant distributed protocols. For instance, $[53,107]$ consider a rich class of protocols but require the user to supply such constraints to automatically establish correctness. The work in [107] abstracts the quorum of threads in the Paxos protocols by introducing a new sort for quorum and adding an axiom to capture the fact that the intersection of two quorums is non-empty. While this abstraction is enough for verifying Paxos, it is too coarse for the protocols we consider. This is because we consider protocols in which the size of the intersection of quorums is essential for proving their correctness. Other approaches [66, 77, 46, 69] can tackle wider classes of systems but adopt an interactive approach to verification, while our approach is fully automatic.

Contributions. We propose a sound and automatic approach to checking safety properties. More specifically:

1. We identify a subclass of fault-tolerant distributed protocols in terms of the Heard-Of model and describe the considered safety properties.

2. We introduce a symbolic representation by which we capture cardinality constraints on multisets (formed by values of the variables or heard-of sets) using integer counters, hence avoiding the challenge of implementing quantifier elimination for theories with cardinality constraints.

3. We show how to use the symbolic representation in a sound but (in general) incomplete procedure for checking state reachability in the presence of lossy or corrupt communication.

4. We show termination of the procedure even in the presence of arbitrarily many processes.

5. We report on our experiments with correct and buggy examples.

Outline. We describe the challenges of the verification problem using an illustrative example in Section 8.2. We then introduce the class of distributed protocols and the properties we aim to verify in Section 8.3. We formalize the symbolic representations in Section 8.4 and use them in Section 8.5 in a sound 
init: $x$, res $=-1$

$r \bmod 1=0$ :

send $\mathrm{x}$;

1. $|\mathrm{HO}|>2 n / 3 \wedge\left|\mathrm{HO}^{1}\right| \leq\left|\mathrm{HO}^{0}\right| \leq 2 n / 3 \rightarrow \mathrm{x}:=0$

2. $|\mathrm{HO}|>2 n / 3 \wedge\left|\mathrm{HO}^{0}\right|<\left|\mathrm{HO}^{1}\right| \leq 2 n / 3 \rightarrow \mathrm{x}:=1$

3. $\left|\mathrm{HO}^{0}\right|>2 n / 3 \rightarrow \mathrm{x}$, res $:=0,0$

4. $\left|\mathrm{HO}^{1}\right|>2 n / 3 \rightarrow \mathrm{x}$, res $:=1,1$

5. $|\mathrm{HO}| \leq 2 n / 3 \rightarrow$ skip

Figure 8.1: One-Third-Rule consensus algorithm. An arbitrary number of processes synchronize in rounds and try to choose the same value for res. HO is the multiset of values received from other processes and $\left|\mathrm{HO}^{\mathrm{d}}\right|$ is the number of those messages that are equal to $d$.

(but in general incomplete) verification procedure for which we show termination. We describe our experiments with our open-source prototype tool that implements the procedure in Section 8.6, and we conclude in Section 8.7.

\subsection{Illustrative Example}

The One-Third-Rule algorithm listed in Figure 8.1 is a simple consensus protocol that can tolerate benign transmission failures such as process crashes and message losses. Each process $\mathrm{p}$ has two local variables $\mathrm{x}_{\mathrm{p}}$ and $\mathrm{res}_{\mathrm{p}}$ that range over finite domains. The values of each variable $x_{p}$ range over the set $\{0,1\}$. They are used to capture the candidate of the process $\mathrm{p}$ in the consensus algorithm. The values of each variable res $_{\mathrm{p}}$ range over $\{-1,0,1\}$ and are used to capture the decision of the process. The initial value -1 captures that the process has not decided yet. The example is formalized in the Heard-Of model where $n$ processes operate in infinite rounds in lock-step. The goal of the protocol is for the processes to agree on one of the initial values as a common output.

In each round, a process first sends its local candidate value $\mathrm{x}_{\mathrm{p}}$ to all other processes and receives values sent by other processes. Then, it executes one of the guarded commands that follow the send operation and whose guard is satisfied. In the original HO model [30], it is assumed that process identifiers of the processes that a process $\mathrm{p}$ hears from are stored in the set $\mathrm{HO}_{\mathrm{p}}$. We make a small modification and assume that only the received values from those processes are stored in a local multiset $\mathrm{HO}_{\mathrm{p}}$ called the heard-of multiset of $\mathrm{p}$. There are as many $\mathrm{HO}_{\mathrm{p}}$ multisets as there are processes at each round. These are used to capture different failures uniformly (e.g., delays, losses, crashes, and corruption). For instance, if $\langle\mathrm{x}\rangle$ is the multiset obtained by collecting the values of all variables $x_{p}$ just sent by all processes, and in case of benign transmission failures (e.g., process crashes or message losses), each 
$\mathrm{HO}_{\mathrm{p}}$ will be smaller than $\langle\mathrm{x}\rangle$ for each value, written $\left.\mathrm{HO}_{\mathrm{p}} \leq<\mathrm{x}\right\rangle$. For a multiset $<\mathrm{m}\rangle$, we write $|<\mathrm{m}\rangle \mid$ to mean the cardinality of $\langle\mathrm{m}\rangle$. For instance, $|<\mathrm{x}\rangle \mid$ is the number of processes running the algorithm (i.e., $n$ ) while $\left|\mathrm{HO}_{\mathrm{p}}\right|$ captures the total number of messages received by process $\mathrm{p}$ (i.e., the total number of processes that $\mathrm{p}$ heard from). Moreover, for any value $\mathrm{d}$ in the domain of the sent variables, we write $\left|\mathrm{HO}_{\mathrm{p}}^{\mathrm{d}}\right|$ to mean the number of those messages that are equal to $d$.

In Figure 8.1, a process $\mathrm{p}$ that receives more messages than two-thirds of the total number of processes (i.e., $\left|\mathrm{HO}_{\mathrm{p}}\right|>2 n / 3$ ) will update the value of its local candidate $\mathrm{x}_{\mathrm{p}}$ with the smallest most often received value (lines 1 to 4 ). Besides that, if among the received messages, more than two-thirds of the number of processes have the same value (here, $\left|\mathrm{HO}_{\mathrm{p}}^{0}\right|>2 n / 3$ or $\left|\mathrm{HO}_{\mathrm{p}}^{1}\right|>2 n / 3$ ), then both variables $x_{p}$ and res $_{p}$ are updated to that value (lines 3 and 4 ). It is then said that the process has decided on the value of $r e s_{p}$. Observe that if a process does not receive its candidate value $\mathrm{x}_{\mathrm{p}}$ from more than $2 n / 3$ processes, then it will not decide on it (lines 1 and 2). Furthermore, if a process has only heard from less than $2 n / 3$ processes, it will not update its local variables (line 5).

Typical safety properties for such consensus protocols include:

- Agreement: whenever two processes have reached a decision, the values they have decided on must be equal.

- Validity: if all processes propose the same initial value, then the processes which reach a decision must have decided on that initial value.

- Irrevocability: if a process decides on a value, it does not revoke its decision later.

Detecting violations of the above properties boils down to checking reachability of sets of configurations for unbounded numbers of processes. However, the correctness of the One-Third-Rule algorithm is independent of the number of processes. In fact, its correctness lies in the fact that: (1) in each round, $\mathrm{HO}_{\mathrm{p}} \leq<\mathrm{x}>$ for each process $\mathrm{p},(2)$ only those processes can update their $\mathrm{x}$ which have heard from more than two thirds of the total number of processes, and (3) only those can decide which have heard the same value from more than two-thirds of the processes.

To capture an unbounded number of processes, we use constraints that group the processes based on the valuations of their local variables. Observe that there are finitely many such valuations. We then describe the bad configurations using such constraints. For instance, to check the agreement property for the One-Third-Rule algorithm, we need to check the reachability of all constraints capturing all configurations where at least two groups of processes, namely $i$ and $j$, have $\operatorname{res}_{i}=0$ and $\operatorname{res}_{j}=1$. Assume this constraint had been reached after $r$ rounds. It is not difficult to see that process groups $i$ and 


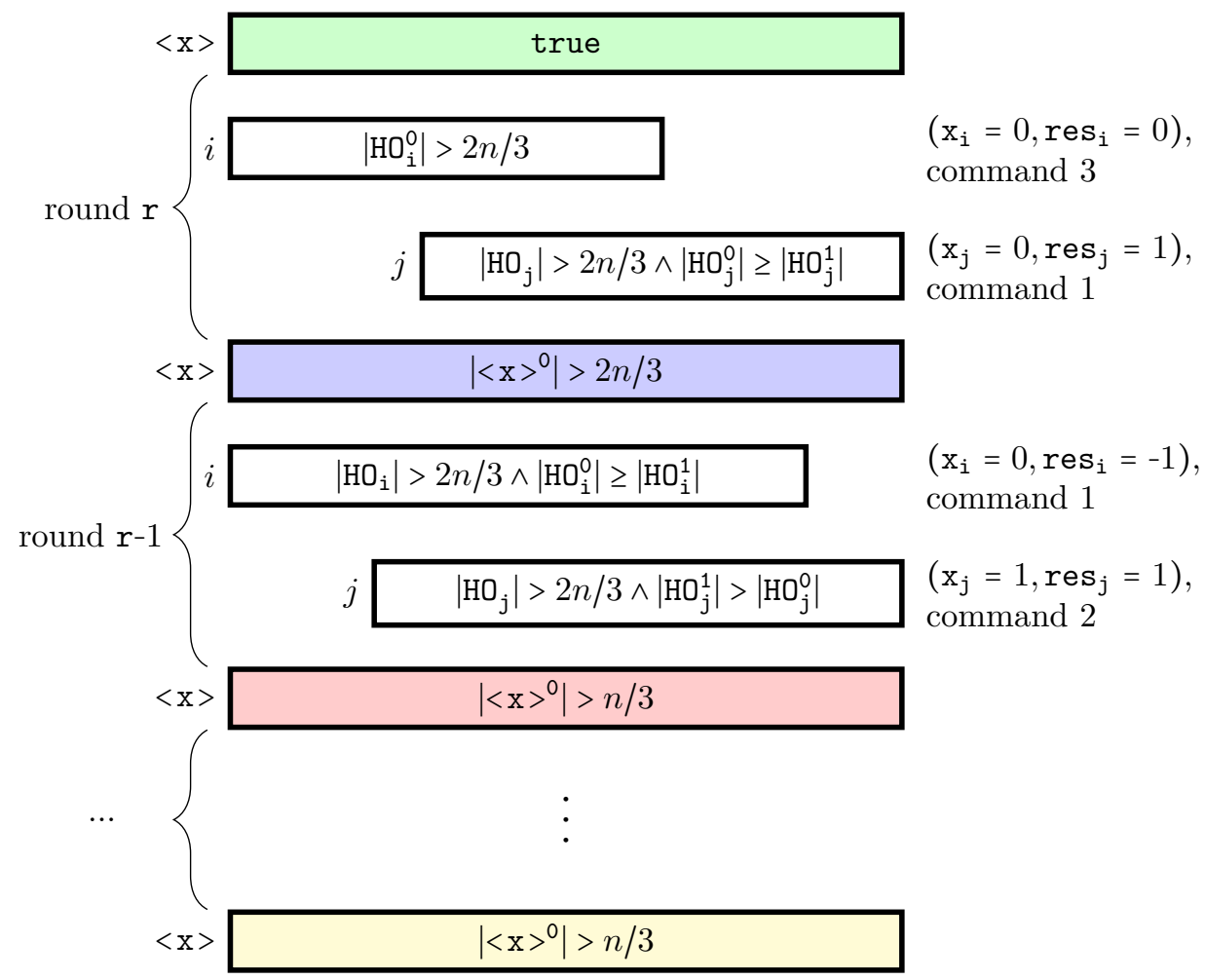

Figure 8.2: A run of the One-Third-Rule algorithm by two process groups i and $j$ in backward analysis. The analysis starts from the configurations with processes that have decided on different values of res. The widths of the bars model the size of the corresponding multisets. Different colors correspond to different rounds.

j could not have executed the guarded commands 3 and 4 during the same round $r$ and assign 0 and 1 to res $_{i}$ and $r e s_{j}$ respectively. This is because they would have had to satisfy both of the guards $\left|\mathrm{HO}_{i}^{0}\right|>2 n / 3$ and $\left|\mathrm{HO}_{j}^{1}\right|>2 n / 3$. Combined with $\left.\mathrm{HO}_{\mathrm{p}} \leq<\mathrm{x}\right\rangle$ for each process $\mathrm{p}$ (since message loss is the only fault), we get $\mid\left\langle\mathrm{x}>^{0}\right|>2 n / 3$ and $\left|\langle\mathrm{x}\rangle^{1}\right|>2 n / 3$. This would give $\mid\langle\mathrm{x}>|>4 n / 3$, which contradicts the assumption that the number of processes in the system is $n$. Thus, what remains is to look for runs in which $r e s_{i}$ and $r e s_{j}$ are set to different values in separate rounds.

Figure 8.2 depicts a possible backward run in which each group of processes in each round is represented by its valuation, its heard-of multiset and the weakest predicate on its local variables that needs to be satisfied to make the run possible. In this description, we do not account for corruption or du- 
plication of messages and therefore assume the heard-of multisets are smaller (because of message loss) than the multiset of the sent values $\langle x\rangle$. Accounting for corruption or duplication of messages is a matter of assuming other relations between $\langle\mathrm{x}\rangle$ and the heard-of multisets. A key to the correctness of the algorithm is the fact that the fraction $2 n / 3$ used in the guards ensures that the local heard-of multisets of the participating processes (i.e., not executing the skip command because they did not receive enough messages) have large intersections (in fact, larger than $n / 3$ for any pair of such multisets).

We start the run without any assumption on $\langle\mathrm{x}\rangle$, therefore it satisfies true. If all processes in the group $i$ and all of those in the group $j$ had executed the commands 3 and 1 respectively during round $r$ (note that each group could have also executed more commands, and we might need to split groups), one of the possible predecessors would be that the same process groups $i$ and $j$ existed with valuations $\operatorname{res}_{\mathrm{i}}=0$ and $\mathrm{res}_{\mathrm{j}}=-1$. Moreover, the predicate $\left|\langle\mathrm{x}\rangle^{0}\right|>2 n / 3$ needs to hold at the beginning of round $r$. This is implied by the guards of the commands 3 and $1,\left|\mathrm{HO}_{\mathrm{i}}^{\circ}\right|>2 n / 3$ and $\left|\mathrm{HO}_{j}\right|>$ $2 n / 3 \wedge\left|\mathrm{HO}_{j}^{1}\right| \leq\left|\mathrm{HO}_{\mathrm{j}}^{0}\right| \leq 2 n / 3$, as well as the invariant $\mathrm{HO}_{\mathrm{p}} \leq<\mathrm{x}>$ for each process $\mathrm{p}$. We could unroll the loop once more, assuming that in round $r-1$ the two process groups had executed commands 1 and 2 respectively and assigned different values to their variables $\mathrm{x}_{\mathrm{i}}$ and $\mathrm{x}_{j}$ (this does not contradict $\left.\left|\langle\mathrm{x}\rangle^{0}\right|>2 n / 3\right)$. The guards of the corresponding commands together with the invariant $\left.\mathrm{HO}_{\mathrm{p}} \leq<\mathrm{x}\right\rangle$ for each process $\mathrm{p}$ entail that the predicate $|<\mathrm{x}\rangle^{0} \mid>n / 3$ held at the beginning of the round $r-1$. Further unrollings of the loop in backward analysis for any number of times will maintain $\left|<x>^{0}\right|>n / 3$. As a result, firing command 4 in some previous iteration would have been impossible as it requires $\left|\mathrm{HO}^{1}\right|>2 n / 3$. This command is, however, needed to reach the initial state. A systematic exploration of similar constraints allows us to conclude the impossibility of deciding on different values.

The work in [23] introduced algorithms in the Heard-Of model in which the received messages might be corrupted. One such algorithm is demonstrated in Figure 8.3. We can handle such algorithms, and the analysis is similar to the case where we have omission faults. The only difference is that the invariant in the presence of corruption faults is that no more than $\alpha$ messages received per round, per process, and data value will be corrupted, i.e., $\left|\mathrm{HO}_{\mathrm{p}}^{\mathrm{d}}\right| \leq|<\mathrm{x}\rangle^{\mathrm{d}} \mid+\alpha$.

Our work proposes a sound but (in general) incomplete algorithm for checking control state reachability for an unbounded number of processes. The algorithm is guaranteed to terminate and starts from a symbolic representation of all bad configurations. It successively computes representations of over-approximations of predecessor configurations. 
init: $x$, res $=-1$

$r \bmod 1=0$ :

send $\mathrm{x}$;

1. $|\mathrm{HO}|>T \wedge\left|\mathrm{HO}^{1}\right| \leq\left|\mathrm{HO}^{0}\right| \leq E \rightarrow \mathrm{x}:=0$

2. $|\mathrm{HO}|>T \wedge\left|\mathrm{HO}^{\circ}\right|<\left|\mathrm{HO}^{1}\right| \leq E \rightarrow \mathrm{x}:=1$

3. $\left|\mathrm{HO}^{\circ}\right|>E \rightarrow \mathrm{x}$, res $:=0,0$

4. $\left|\mathrm{HO}^{1}\right|>E \rightarrow \mathrm{x}$, res $:=1,1$

5. $|\mathrm{HO}| \leq T \rightarrow$ skip

Figure 8.3: $\mathcal{A}_{E, T}$ consensus algorithm [23]. An arbitrary number of processes synchronize in rounds and try to choose the same value for res. The messages might get lost or corrupted. Per each round, process, and data value, there will be at most $\alpha$ corrupted messages. $T$ is the threshold on the number of received messages and $E$ is a high enough number of received messages with a certain value. According to [23], for correctness of the algorithm, it is sufficient that $T \geq 2(n+2 \alpha-E), E \geq \frac{n}{2}+\alpha$ and $n>T \geq E$. We check correctness by adding these predicates as invariants.

\subsection{Heard-of Programs}

To simplify the presentation, we use a unique domain for all local variables and assume programs to proceed in infinite rounds where the state of each process is captured by the local variables. Introducing specific data domains for each variable or explicit local states in the transitions is straightforward. We use valuations (i.e., mapping from the set of local variables of a process to its domain) to capture the values of process variables. We define the syntax and semantics of a language to capture a class of heard-of programs. A heard-of program $\operatorname{Pr}=(\mathrm{B}, \mathrm{P}, \mathrm{Ts})$ involves:

- A set V of variables local to each process.

- A finite set $\mathrm{D} \subset \mathbb{Z}$ of possible data values,

- An initial set of valuations Init sending local variables $\mathrm{V}$ to $\mathrm{D}$,

- A set of transitions Tr.

The syntax of such programs is as follows.

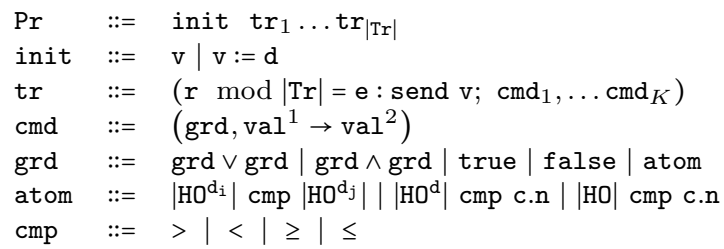


Each process starts by setting initial values to its local variables. Then, all processes execute the transitions in a lock-step manner. Each program consists of a macro-round which is a sequence of $|\mathrm{Tr}|$ consecutive rounds ( $r$ $\bmod |\operatorname{Tr}|)=0, \ldots,(r \bmod |\operatorname{Tr}|)=|\operatorname{Tr}|-1$. The program starts in round $r=0$ and at each round $r$, all of the processes will execute the transition designated with $(r \bmod |\operatorname{Tr}|) . r$ is incremented after each transition.

In each transition $\left(r \bmod |\operatorname{Tr}|=\mathrm{e}:\right.$ send $\mathrm{v} ; \mathrm{cmd}_{1}, \ldots \mathrm{cmd} K$ ), first, all processes send the value of their local variable v. After send, there is an implicit receive step in which the processes receive the values sent by others. Between the send and receive of the messages, an adversarial environment can choose to drop or alter messages. The received values are stored in a Ho (heardof) multiset that is local to each process. The impact of the environment is captured by the heard-of multiset.

After send and receive, each process $\mathrm{p}$ with heard-of multiset $\mathrm{HO}_{\mathrm{p}}$ executes a guarded command $\mathrm{cmd}_{k}=\left(\operatorname{grd}_{k}: \operatorname{val}_{k}^{1} \rightarrow \operatorname{val}_{k}^{2}\right)$ where $\mathrm{HO}_{\mathrm{p}} \vDash \operatorname{grd}_{k}$. A grd mainly focuses on capturing cardinality of some HO multiset(s). In many cases, this cardinality is compared to a fraction of the total number of processes, i.e., c.n. In order to simplify the presentation, we consider each cmd to be a change in the local valuation of a process. A skip command can easily be transformed to this format by choosing identical values for val ${ }^{1}$ and $\mathrm{val}^{2}$ in the command. Introducing explicit local states is simple but would not improve readability.

Configurations. Configurations of a heard-of program consist of the round number, as well as the local state of the processes, i.e., their valuations and heard-of multisets. More formally, a configuration $c=\left(r,<p_{1}, \ldots, p_{a}>\right)$ of $\operatorname{Pr}=(\mathrm{B}, \mathrm{P}, \mathrm{Ts})$ is a tuple in which:

- $a$ is the number of processes in the configuration,

- $r$ is the round number,

- for all $i$ in $0 \leq i \leq a, \mathrm{p}_{i}=\left(\operatorname{val}_{i}, \mathrm{HO}_{i}\right)$ is a process where:

- $\mathrm{val}_{i}$ is a mapping $\mathrm{V} \rightarrow \mathrm{D}$. In other words, the valuation $\mathrm{val}_{i}$ maps each local variable of the process to a value in the domain,

$-\mathrm{HO}_{i}: \mathrm{D} \rightarrow \mathbb{N}$ is a multiset of integer values.

Values of a configuration. For a configuration $c=\left(r,\left\langle\mathrm{p}_{1}, \ldots, \mathrm{p}_{a}\right\rangle\right)$ and for any variable $\mathrm{v} \in \mathrm{V}$ we define valuesOf(c,v) to be a multiset containing all the local values of $\mathrm{v}$ in all the processes. More formally, for all $\mathrm{d} \in \mathrm{D}$, valuesOf $(\mathrm{c}, \mathrm{v})(\mathrm{d})=\mid\left\{\mathrm{p}_{i} \mid \mathrm{p}_{i}=\left(\mathrm{val}_{i}, \mathrm{HO}_{i}\right)\right.$ with $\left.\operatorname{val}_{i}(\mathrm{v})=\mathrm{d}\right\} \mid$.

Example 16. For the program in Figure 8.1, consider the following processes at round $r=0$. 
- $\mathrm{p}_{1}=\left(\left(\mathrm{x}_{1}=1, \mathrm{r}_{1}=-1\right), \varnothing\right)$

- $\mathrm{p}_{2}=\left(\left(\mathrm{x}_{2}=1, \mathrm{r}_{2}=-1\right), \varnothing\right)$

- $\mathrm{p}_{3}=\left(\left(\mathrm{x}_{3}=0, \mathrm{r}_{3}=-1\right), \varnothing\right)$

- $\mathrm{p}_{4}=\left(\left(\mathrm{x}_{4}=1, \mathrm{r}_{4}=-1\right), \varnothing\right)$

The configuration $\mathrm{c}=\left(0,<\mathrm{p}_{1}, \mathrm{p}_{2}, \mathrm{p}_{3}, \mathrm{p}_{4}>\right)$ captures an initial configuration. The heard-of multisets of the processes are empty initially. The values of variable $\mathrm{x}$ are captured by the multiset values $0 \mathrm{f}(\mathrm{c}, \mathrm{x})=<0,1,1,1>$. We have that valuesof $(\mathrm{c}, \mathrm{x})(1)=3$.

Semantics. Given a program $\operatorname{Pr}=(B, P, T s)$, the processes start executing $\operatorname{Tr}$ from an initial configuration $\mathrm{c}_{\text {init }}=\left(\mathrm{r}^{\text {init }},\left\langle\mathrm{p}_{1}^{\text {init }}, \ldots, \mathrm{p}_{a}^{\text {init }}>\right)\right.$ where $\mathrm{r}^{\text {init }}=0$, and for all $1 \leq i \leq a, \mathrm{p}_{i}^{\text {init }}=\left(\mathrm{val}_{i}, \varnothing\right)$, and $\mathrm{val}_{i} \in$ Init. Suppose configurations $\mathrm{c}$ and $\mathrm{c}^{\prime}$ can be written (up to a renaming of the processes) as $\mathrm{c}=$ $\left(\mathrm{r},<\left(\operatorname{val}_{1}, \mathrm{HO}_{1}\right), \ldots,\left(\operatorname{val}_{a}, \mathrm{HO}_{a}\right)>\right), \mathrm{c}^{\prime}=\left(\mathrm{r}^{\prime},<\left(\mathrm{val}_{1}^{\prime}, \mathrm{HO}_{1}^{\prime}\right), \ldots,\left(\mathrm{val}_{a}^{\prime}, \mathrm{HO}_{a}^{\prime}\right)>\right.$ ), and $\operatorname{tr}=\left(\mathrm{r} \bmod |\operatorname{Tr}|=\mathrm{e}:\right.$ send $\left.\mathrm{v} ; \mathrm{cmd}_{1}, \ldots \mathrm{cmd}_{K}\right)$ with $\mathrm{cmd}_{k}=$ $\left(\operatorname{grd}_{k}, \mathrm{val}_{k}^{1} \rightarrow \mathrm{val}_{k}^{2}\right)$ for each $k: 1 \leq k \leq K$. We write $\mathrm{c} \stackrel{\text { tr }}{\rightarrow} \mathrm{c}^{\prime}$ to mean that $\mathrm{r}^{\prime}=\mathrm{r}+1$ and there is a total function $\mathrm{F}:\{1, \ldots, a\} \rightarrow\{1, \ldots, K\}$ where for each $i: 1 \leq i \leq a, \operatorname{val}_{i}=\operatorname{val}_{\mathrm{F}(i)}^{1}, \operatorname{val}_{i}^{\prime}=\operatorname{val}_{\mathrm{F}(i)}^{2}$ and $\mathrm{HO}_{i} \vDash \operatorname{grd}_{\mathrm{F}(i)}$. Note that the numbers of processes in $c$ and $c^{\prime}$ are finite, arbitrarily large and equal.

Example 17. Consider the Example 16 and the transition tr to be the transition tr in Figure 8.1. Processes 1 to 4 can take guarded commands 2, 2, 5 , and 4 , respectively and result in the configuration $\left.c^{\prime}=\left(1,<\mathrm{p}_{1}^{\prime}, \mathrm{p}_{2}^{\prime}, \mathrm{p}_{3}^{\prime}, \mathrm{p}_{4}^{\prime}\right\rangle\right)$ where:

- $\mathrm{p}_{1}^{\prime}=\left(\left(\mathrm{x}_{1}=1, \mathrm{r}_{1}=-1\right),<1,0,1>\right)$

- $\mathrm{p}_{2}^{\prime}=\left(\left(\mathrm{x}_{2}=1, \mathrm{r}_{2}=-1\right),<1,1,0>\right)$

- $\mathrm{p}_{3}^{\prime}=\left(\left(\mathrm{x}_{3}=0, \mathrm{r}_{3}=-1\right),<0,1>\right)$

- $\mathrm{p}_{4}^{\prime}=\left(\left(\mathrm{x}_{4}=1, \mathrm{r}_{4}=1\right),<1,1,1>\right)$

Here, $\mathrm{F}=\{(1,2),(2,2),(3,5),(4,4)\}$ witnesses $\mathrm{c} \stackrel{\operatorname{tr}}{\longrightarrow} \mathrm{c}^{\prime}$.

\subsection{Symbolic Representation}

In this section, we formally define our symbolic representation and describe a corresponding entailment relation. We assume a program $\mathrm{Pr}=(\mathrm{B}, \mathrm{P}, \mathrm{Ts})$. 
Constraints. A constraint $\varphi$ is a tuple $\left(\mathrm{e}, \mathrm{gl},\left\{\mathrm{val}_{1}, \ldots, \mathrm{val}_{b}\right\}\right)$ that denotes a possibly infinite set of configurations such that:

- An integer e in $\{0, \ldots,|\operatorname{Tr}|-1\}$ captures the control location of the execution.

- A global condition gl in the form of a Presburger predicate with a free variable $n$ (for the number of processes) and a set of $|\mathrm{V}| \times|\mathrm{D}|$ free variables ${ }^{\#} \mathrm{~V}=\left\{{ }^{\#} \mathrm{~V}^{\mathrm{d}} \mid \mathrm{v} \in \mathrm{V}\right.$ and $\left.\mathrm{d} \in \mathrm{D}\right\}$, where each ${ }^{\#} \mathrm{v}^{\mathrm{d}}$ accounts for the number of occurrences of value $\mathrm{d}$ among the variables $\mathrm{v}$ of all processes.

- A base formed by a set of valuations $\left\{\operatorname{val}_{1}, \ldots, \operatorname{val}_{b}\right\}$. The valuations are similar to those used by the configurations and represent groups of processes with the same valuations.

Each valuation in the base of a constraint corresponds to one or more processes with that valuation in a denoted configuration. Besides, a constraint does not explicitly represent conditions on heard-of multisets; instead, it maintains a global condition gl which is a predicate on the number of occurrences of values of the local variables of all processes (i.e. global state). The intuition is that the heard-of sets ultimately originate from the global state. Hence, constraining their values (to satisfy some guarded commands) is a matter of constraining the global state and accounting for possible faults (see Section 8.5). For a predicate $p$ that might depend on a set of integer variables $X=\left\{x_{1}, \ldots, x_{q}\right\}$, we write $p(X)$ to clarify that $p$ might have a subset of $X$ as free variables. To simplify the presentation, we typically do not mention that a predicate might have $n$ (for the number of processes) as a free variable. For instance, we write $g l\left({ }^{\#} \mathrm{~V}\right)$ to clarify that $\mathrm{gl}$ might have a subset of ${ }^{*} \mathrm{~V}$ in addition to the variable $n$ as its free variables .

Denotations. For a constraint $\varphi=\left(\mathrm{e}, \mathrm{gl}^{\varphi},\left\{\operatorname{val}_{1}^{\varphi}, \ldots, \mathrm{val}_{b}^{\varphi}\right\}\right)$ we write $\mathrm{c} \vDash$ $\varphi$ to mean $\varphi$ denotes the configuration $\mathrm{c}=\left(\mathrm{r},\left\langle\left(\operatorname{val}_{1}^{\mathrm{c}}, \mathrm{HO}_{1}^{\mathrm{c}}\right), \ldots,\left(\mathrm{val}_{a}^{\mathrm{c}}, \mathrm{HO}_{a}^{\mathrm{c}}\right)\right\rangle\right.$ ). Intuitively, $\varphi$ should account for all local valuations in $c$ (captured by a surjection from $\{1, \ldots, a\}$ to $\{1, \ldots, b\})$. Moreover, the predicate gl ${ }^{\varphi}$ must be compatible with the multiset of all local valuations of the processes. More formally:

1. $r \bmod |\operatorname{Tr}|=\mathrm{e}$.

2. Replacing in the global condition gl each occurrence of ${ }^{\#} \mathrm{v}^{\mathrm{d}}$ with the number of occurrences of $d$ in $c$ (i.e., valuesOf $(c, v)(d)$ ) and each occurrence of $n$ with the number of processes in c (i.e., $a)$ results in a satisfiable formula.

3. There is a surjection $\operatorname{Sr}::\{1, \ldots, a\} \rightarrow\{1, \ldots, b\}$ such that for all $1 \leq i \leq$ $a, \operatorname{val}_{i}^{\mathrm{c}}=\operatorname{val}_{\mathrm{Sr}(i)}^{\varphi}$ 
Observe that a constraint $\left(\mathrm{e}, \mathrm{gl},\left\{\mathrm{val}_{1}, \ldots, \mathrm{val}_{b}\right\}\right)$ will have an empty denotation if its base requires the presence of valuations forbidden by the global condition, or if the global condition requires valuations forbidden by the base (since we require a surjection). It is safe to systematically discard such constraints in our analysis presented in Sec. 8.5.

Example 18. The configuration $c^{\prime}$ in Example 17 is in the denotation of the constraint $\varphi^{\prime}=\left(0,{ }^{\#} \mathrm{x}^{1}>2 n / 3,\left\{\left(\mathrm{x}_{1}=1, \mathrm{r}_{1}=-1\right),\left(\mathrm{x}_{1}=0, \mathrm{r}_{2}=-1\right),\left(\mathrm{x}_{3}=1, \mathrm{r}_{3}=1\right)\right\}\right)$ with $\mathrm{Sr}=\{(1,1),(2,1),(3,2),(4,3)\}$.

Entailment. We write $\varphi_{1} \sqsubseteq \varphi_{2}$ to mean $\varphi_{1}=\left(\mathrm{e}, \mathrm{gl}^{1},\left\{\operatorname{val}_{1}^{1}, \ldots, \operatorname{val}_{b_{1}}^{1}\right\}\right)$ is entailed by $\varphi_{2}=\left(\mathrm{e}, \mathrm{gl}^{2},\left\{\mathrm{val}_{1}^{2}, \ldots, \mathrm{val}_{b_{2}}^{2}\right\}\right)$. This will ensure that configurations denoted by $\varphi_{2}$ are also denoted by $\varphi_{1}$. Intuitively, $\varphi_{1}$ and $\varphi_{2}$ must have the same round number modulo $|\operatorname{Tr}|$, and

- There is a bijection Y :: $\left\{1, \ldots, b_{2}\right\} \rightarrow\left\{1, \ldots b_{1}\right\}$ with $\operatorname{val}_{j}^{2}=\operatorname{val}_{\mathrm{Y}(j)}^{1}$ for all $1 \leq j \leq b_{2}$.

- $\mathrm{gl}^{2} \Rightarrow \mathrm{gl}^{1}$.

\subsection{A Symbolic Verification Procedure}

We use the constraints defined in Section 8.4 as a symbolic representation to denote sets of configurations. We adapt the working-list procedure introduced in Section 3.3 to check reachability of a ㄷ-minimal set $\Phi$ of target constraints by a program $\operatorname{Pr}=(B, P, T s)$. For a bad set $B=\left\{\operatorname{val}_{1}, \ldots, \mathrm{val}_{x}\right\}$ of valuations, the set of target constraints $\Phi_{B}$ contains each $\left(e, t r u e, v l_{1}, \ldots, v l_{x}\right)$ where $\mathrm{e}$ is a value in $\{0, \ldots,|\operatorname{Tr}|-1\}$. In addition, it contains each constraint obtained from such a constraint by adding some unique valuations that were not in its base (since we require surjections for the denotations in Section 8.4).

The procedure computes a fixpoint using the entailment relation $\subseteq$ and a predecessor computation that results, for a constraint $\varphi$ and a transition tr, in a finite set $\operatorname{pre}_{\mathrm{tr}}(\varphi)$. In fact, $\operatorname{pre}_{\mathrm{tr}}(\varphi)$ is the set of constraints that capture an over-approximation of all the configurations that might reach a configuration that is denoted by $\varphi$ in one round. Figure 8.4 captures this computation and uses several sets of integer variables. The variables ${ }^{\#} \mathrm{~V}=\left\{{ }^{\#} \mathrm{~V}^{\mathrm{d}} \mid \mathrm{v} \in \mathrm{V}\right.$ and $\left.\mathrm{d} \in \mathrm{D}\right\}$ (resp. ${ }^{\#} \mathrm{~V}^{\prime}=\left\{{ }^{*} \mathrm{v}^{\prime \mathrm{d}} \mid \mathrm{v} \in \mathrm{V}\right.$ and $\left.\mathrm{d} \in \mathrm{D}\right\}$ ) are used to constrain values of process variables in the successor constraint $\varphi$ (resp. predecessor constraint $\varphi^{\prime}$ ). The variables ${ }^{\#} \mathrm{HO}_{k}=\left\{{ }^{\#} \mathrm{ho}_{\mathrm{k}}^{\mathrm{d}} \mid \mathrm{d} \in \mathrm{D}\right\}$ are used to constrain values in the heard-of multisets of processes taking a guarded command $\mathrm{cmd}_{k}$ in tr. The remainder of this section describes the over-approximation.

Choice of guarded commands and resulting bases. Intuitively, the set I in Figure 8.4 corresponds to combinations of processes in the successors (i.e., 


$$
\begin{aligned}
& \varphi=\left(\mathrm{e}, \mathrm{gl},\left\{\mathrm{val}_{1}, \ldots, \mathrm{val}_{b}\right\}\right) \\
& \mathrm{tr}=\left(\mathrm{r} \bmod |\mathrm{Tr}|=(\mathrm{e}-1): \text { send } \mathrm{v} ; \mathrm{cmd}_{1}, \ldots, \mathrm{cmd}_{K}\right) \\
& 1 \leq k \leq K \Longrightarrow \mathrm{cmd}_{k}=\left(\operatorname{grd}_{k}, \mathrm{val}_{k}^{1} \rightarrow \mathrm{val}_{k}^{2}\right) \\
& \mathrm{I} \subseteq\{1, \ldots, K\} \times\{1, \ldots, b\} \text { st. } \mathrm{I}_{\{1, \ldots, b\}}=\{1, \ldots, b\} \\
& \mathrm{H}::\{1, \ldots,|\mathrm{I}|\} \rightarrow \mathrm{I} \text { is an enumeration of } \mathrm{I} \\
& 1 \leq i \leq|\mathrm{I}| \wedge \mathrm{H}(i)=(k, j) \Longrightarrow \operatorname{val}_{i}^{\prime}=\operatorname{val}_{k}^{1} \wedge \operatorname{val}_{k}^{2}=\operatorname{val}_{j} \\
& \Gamma=\left\{\gamma_{k} \mid k: 1 \leq k \leq K\right\} \text { with } \\
& \begin{array}{c}
\gamma_{k}\left({ }^{\#} \mathrm{~V}^{\prime}\right)=\exists^{\#} \mathrm{HO}_{k} \cdot \xi\left(\operatorname{grd}_{k}\right)\left({ }^{\#} \mathrm{HO}_{k}\right) \wedge \operatorname{HAX}_{k}\left({ }^{\#} \mathrm{~V}^{\prime}\right)=\left(\operatorname{Inv} \wedge \wedge_{(k, \ldots) \in \mathrm{I}} \gamma_{k}\left({ }^{\#} \mathrm{~V}^{\prime}\right) \wedge \operatorname{PrAbs}_{[\Gamma]}\left({ }^{\#}\right)\right. \\
\left.\left.\mathrm{V} \cdot \operatorname{DAX}\left({ }^{\#} \mathrm{~V},{ }^{\#} \mathrm{~V}^{\prime}\right) \wedge \mathrm{gl}\left({ }^{\#} \mathrm{~V}\right)\right)\right)
\end{array} \\
& \operatorname{Inv}=\left(\sum_{\mathrm{d} \in \mathrm{D}}^{\#} \mathrm{v}^{\prime}=n\right) \\
& \varphi^{\prime}=\left((\mathrm{e}-1) \bmod |\mathrm{Tr}|, \mathrm{gl}^{\prime}\left[{ }^{\#} \mathrm{~V}^{\prime} /{ }^{\#} \mathrm{~V}\right], \operatorname{set} 0 \mathrm{f}\left(\mathrm{val}_{1}^{\prime}, \ldots, \mathrm{val}_{|\mathrm{I}|}^{\prime}\right)\right) \\
& \varphi^{\prime} \in \operatorname{pre}_{\text {tr }}(\varphi)
\end{aligned}
$$

Figure 8.4: Predecessor computation for a constraint $\varphi$ and a transition tr

$\varphi)$ and guarded commands in the transition (i.e., tr). Each pair $(k, j) \in \mathrm{I}$ corresponds to a group of processes with the same valuation $\operatorname{val}_{H^{-1}((k, j))}^{\prime}$ in the predecessors (i.e., $\varphi^{\prime}$ ) that took the guarded command $\mathrm{cmd}_{k}$ in the transition $\operatorname{tr}$ resulting in a valuation $\operatorname{val}_{j}$ in $\varphi$. Observe that there are finitely many such combinations, and hence, finitely many such sets I. The definition of I ensures that the set $\{1, \ldots, b\}$ of process groups of $\varphi$ is covered. In addition, two pairs $\left(k_{1}, j_{1}\right)$ and $\left(k_{2}, j_{2}\right)$ may result in equal valuations $\operatorname{val}_{H^{-1}\left(\left(k_{1}, j_{1}\right)\right)}^{\prime}$ and $\operatorname{val}_{H^{-1}\left(\left(k_{2}, j_{2}\right)\right)}^{\prime}$. We keep only one representative in $\varphi^{\prime}$ by making a set set0f $\left(\operatorname{val}_{1}^{\prime}, \ldots, \operatorname{val}_{|\mathrm{I}|}^{\prime}\right)$ of the multiset $\left\langle\operatorname{val}_{1}^{\prime}, \ldots, \operatorname{val}_{|\mathrm{I}|}^{\prime}\right\rangle$.

Constraints imposed by the guards. For a guarded command $\mathrm{cmd}_{k}$, we use the predicate $\xi\left(\operatorname{grd}_{k}\right)$ to encode the fact that the heard-of multisets of the predecessor configurations denoted by $\varphi^{\prime}$ satisfy the guard $\operatorname{grd}_{k}$ of $\mathrm{cmd}_{k}$. For this, we use an integer variable ${ }^{\#}{ }_{\mathrm{ho}}^{\mathrm{d}}$ for each value $\mathrm{d}$ and index $k: 1 \leq k \leq K$ to count the occurrences of $d$ in the heard-of multiset of the processes taking $\mathrm{cmd}_{k}$. We write ${ }^{\#} \mathrm{HO}_{k}=\left\{{ }^{\#} \mathrm{ho}_{\mathrm{k}}^{\mathrm{d}} \mid \mathrm{d} \in \mathrm{D}\right\}$ to mean the set of all such variables for all values in D. For instance, $\operatorname{grd}_{3}$ is $\left|\mathrm{HO}^{\circ}\right|>2 n / 3$ in Figure 8.1 and is encoded with the predicate $\left({ }^{\#} \mathrm{ho}_{3}^{0}>2 n / 3\right)$. We also need to relate the constraints on the heard-of multisets to the constraints on the variables' values in the predecessor constraint $\varphi^{\prime}$. Assume $\varphi^{\prime}$ denotes a configuration $c^{\prime}$ resulting, via $t r$, in a configuration $\mathrm{c}$ denoted by $\varphi$. Suppose $\operatorname{tr}$ sends values of variable v. In the case of benign failures (e.g., message losses), any heardof multiset $\mathrm{HO}_{k}$ of some process that took a guarded command $\mathrm{cmd}_{k}$ in $\mathrm{tr}$ needs to get its values from the multiset valuesOf $\left(c^{\prime}, v\right)$. Recall that the multiset valuesOf $\left(c^{\prime}, v\right)$ is the multiset over the values of $v$ in the configu- 
ration $c^{\prime}$. Therefore, we enforce $\mathrm{HO}_{k} \leq \operatorname{values} \mathrm{Of}\left(\mathrm{c}^{\prime}, \mathrm{v}\right)$. This is captured by $\operatorname{HAX}_{k}\left({ }^{\#} \mathrm{HO}_{k},{ }^{\#} \mathrm{~V}^{\prime}\right)=\bigwedge_{\mathrm{d} \in \mathrm{D}} 0 \leq{ }^{\#} \mathrm{ho}_{\mathrm{k}}^{\mathrm{d}} \leq{ }^{\#} \mathrm{v}^{\prime}{ }^{\mathrm{d}}$. For each guarded command $\mathrm{cmd}_{k}$, the predicate $\gamma_{k}\left({ }^{\#} \mathrm{~V}^{\prime}\right)=\exists^{\#} \mathrm{HO}_{k} \cdot\left(\xi\left(\operatorname{grd}_{k}\right)\left({ }^{\#} \mathrm{HO}_{k}\right) \wedge \operatorname{HAX}_{k}\left({ }^{\#} \mathrm{HO}_{k},{ }^{\#} \mathrm{~V}^{\prime}\right)\right)$ captures the strongest constraints imposed, in the predecessor processes, by the guard of $\mathrm{cmd}_{k}$ on values of the variable that was sent (here, v). We explain later in this section how we handle corrupt communication. These predicates only depend on the chosen guarded commands and the sent variables. We collect them in a set $\Gamma=\left\{\gamma_{k} \mid k: 1 \leq k \leq K\right\}$. Observe that the set $\Gamma$ is finite.

Constraints imposed by the commands. Each time a process takes a chosen guarded command $\mathrm{cmd}_{k}=\left(\operatorname{grd}_{k}, \operatorname{val}_{k}^{1} \rightarrow \operatorname{val}_{k}^{2}\right)$ with $(k, j)$ in I for some $j$, it transforms its valuation from $\operatorname{val}_{k}^{1}$ to $\mathrm{val}_{k}^{2}$. This affects the relation between $\mathrm{gl}\left({ }^{\#} \mathrm{~V}\right)$ and $\mathrm{gl}^{\prime}\left({ }^{\#} \mathrm{~V}^{\prime}\right)$ as the number of occurrences of a variable with a given value depends on the proportions of the processes that take each guarded command. We first illustrate how this relation can be captured exactly by introducing auxiliary variables to represent the number of processes that took each one of the chosen guarded commands. Then, we describe how we can over-approximate this relation and avoid the introduction of those variables.

First, we introduce an integer variable $\delta_{k}$, for each $k \in\{1, \ldots, K\}$, to capture the number of processes in some configuration $c^{\prime}$ denoted by $\varphi^{\prime}$ that executed the guarded command $\mathrm{cmd}_{k}=\left(\operatorname{grd}_{k}, \operatorname{val}_{k}^{1} \rightarrow \operatorname{val}_{k}^{2}\right)$. If $\mathrm{d}_{1}=\operatorname{val}_{k}^{1}(\mathrm{v})$ and $\mathrm{d}_{2}=\mathrm{val}_{k}^{2}(\mathrm{v})$, then, each process that takes the guarded command $\mathrm{cmd}_{k}$ will decrease the number of occurrences of the value $d_{1}$ among messages and increase the number of occurrences of $d_{2}$. More precisely, for each variable $v$, the following relation holds:

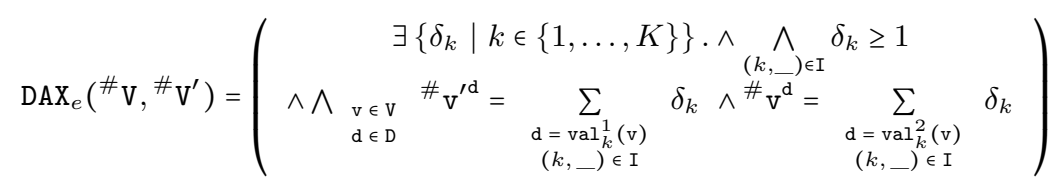

The relation $\mathrm{DAX}_{e}$ is expensive to compute. Instead, we over-approximate it with DAX (see below) where we identify two cases in which we can relate variables in ${ }^{\#} \mathrm{~V}$ and ${ }^{\#} \mathrm{~V}^{\prime}$. For each variable $\mathrm{v} \in \mathrm{V}$, the first case (captured with the predicate $\left.\operatorname{preserved}_{\mathrm{I}}(\mathrm{v})\right)$ is true when each chosen guarded command $\left(\operatorname{grd}_{k}, \operatorname{val}_{k}^{1} \rightarrow \operatorname{val}_{k}^{2}\right)$ with $(k, \ldots) \in \mathrm{I}$ preserves the variable $\mathrm{v}$ (i.e., $\operatorname{val}_{k}^{1}(\mathrm{v})=$ $\left.\mathrm{val}_{k}^{2}(\mathrm{v})\right)$. The second case (captured with the predicate uniqueChange $\mathrm{I}_{\mathrm{I}}(\mathrm{v}, \mathrm{d})$ ) corresponds to the situation when the only allowed changes for variable $\mathrm{v}$ are to assign some value $\mathrm{d}$ to it. Formally, for all $k, k^{\prime}$ with $(k, \ldots),\left(k^{\prime}, \ldots\right) \in \mathrm{I}$, if $\operatorname{val}_{k}^{1}(\mathrm{v}) \neq \operatorname{val}_{k}^{2}(\mathrm{v})$ and $\operatorname{val}_{k^{\prime}}^{1}(\mathrm{v}) \neq \operatorname{val}_{k^{\prime}}^{2}(\mathrm{v})$, then, $\operatorname{val}_{k}^{2}(\mathrm{v})=\operatorname{val}_{k^{\prime}}^{2}(\mathrm{v})=\mathrm{d}$. The over-approximation $\mathrm{DAX}$ of $\mathrm{DAX}_{e}$ is defined as: 


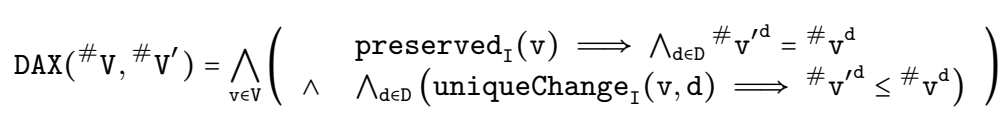

To compute $g l^{\prime}\left({ }^{\#} \mathrm{~V}^{\prime}\right)$, we start from the global condition of the successor constraint (i.e., $\left.\mathrm{gl}\left({ }^{\#} \mathrm{~V}\right)\right)$ and deduce constraints on $\mathrm{V}^{\prime}$ via the relation $\operatorname{DAX}\left({ }^{\#} \mathrm{~V},{ }^{\#} \mathrm{~V}^{\prime}\right)$. More precisely, we compute: $\pi\left({ }^{\#} \mathrm{~V}^{\prime}\right)=\exists^{\#} \mathrm{~V} \cdot \operatorname{DAX}\left({ }^{\#} \mathrm{~V},{ }^{\#} \mathrm{~V}^{\prime}\right) \wedge$ $\mathrm{gl}\left({ }^{\#} \mathrm{~V}\right)$. In general, arbitrarily many different such predicates may be generated in the fixpoint iteration. To help termination, we use the abstraction $\operatorname{PrAbs}_{[\Gamma]}(\pi)$ of $\pi$ with respect to the predicates $\Gamma=\left\{\gamma_{k} \mid k: 1 \leq k \leq K\right\}$ obtained from all the guards of the commands.

Example 19. Consider the configurations c, $c^{\prime}$ and the constraint $\varphi^{\prime}$ in the Examples 16, 17 and 18. We have shown that $c \stackrel{\text { tr }}{\rightarrow} c^{\prime}$ using the mapping $\mathrm{F}$ and $\mathrm{c}^{\prime} \vDash \varphi^{\prime}$ using the surjection Sr. Consider now the constraint $\varphi=$ $\left(0,{ }^{\#} \mathrm{x}^{1}>2 n / 3,\left\{\left(\mathrm{x}_{1}=1, \mathrm{r}_{1}=-1\right),\left(\mathrm{x}_{1}=0, \mathrm{r}_{2}=-1\right)\right\}\right)$. We define the mapping $\mathrm{H}=\{(1,(2,1)),(2,(5,2)),(1,(4,3))\}$ to show $\varphi^{\prime} \in \operatorname{pre}_{\mathrm{tr}}(\varphi)$. Moreover, there is a surjection $\operatorname{Sr}^{\prime}=\{(1,1),(2,1),(3,2),(4,1)\}$ that witnesses $\mathrm{c} \vDash \varphi$.

Corrupted communications. Corrupted communications are related to the classical Byzantine Faults in which a portion of the received messages are corrupted [23]. Note that in the classical Byzantine setting, the state of a process can also be corrupted, which is not the case in this model. All processes follow the algorithm but may receive some corrupted messages (whether accidental or malicious). We assume that in each round, for each process and each data value, no more than $\alpha$ (given as a fraction of $n$ ) messages received by a process may have been corrupted. This assumption is weaker than the one in [23]. We model this fault by enforcing the following invariants. DAX remains unchanged because of the assumption that states of the processes are not corrupted. It is the relation between the heard-of multisets and process variables that changes: $\operatorname{HAX}_{k}=\bigwedge_{\mathrm{d} \in \mathrm{D}}\left(0 \leq{ }^{\#} \mathrm{ho}_{\mathrm{k}}^{\mathrm{d}} \leq{ }^{\#} \mathrm{v}^{\prime}{ }^{\mathrm{d}}+\alpha\right)$. The rest of the computation of predecessors also remains unchanged.

Theorem 23. The proposed predecessor computation method introduced in Figure 8.4 is a sound over-approximation for parameterized Heard-Of programs.

Proof. Assume two configurations $\mathrm{c}=\left(\mathrm{r},<\mathrm{p}_{1}, \ldots, \mathrm{p}_{a}>\right), \quad \mathrm{c}^{\prime}=$ $\left(\mathrm{r}^{\prime},<\mathrm{p}_{1}^{\prime}, \ldots, \mathrm{p}_{a}^{\prime}>\right)$, a constraint $\varphi$, and a transition $\operatorname{tr}=$ $\left(\mathrm{r} \bmod |\operatorname{Tr}|=(\mathrm{e}-1):\right.$ send $\left.\mathrm{v} ; \mathrm{cmd}_{1}, \ldots, \mathrm{cmd}_{K}\right)$ with $\mathrm{cmd}_{k}=\left(\operatorname{grd}_{k}, \mathrm{val}_{k}^{1} \rightarrow \operatorname{val}_{k}^{2}\right)$ for each $k: 1 \leq k \leq K$ as described in Figure 8.5.

The total function $F:\{1, \ldots, a\} \rightarrow\{1, \ldots, K\}$ witnesses $c^{\prime} \stackrel{\text { tr }}{\rightarrow}$ c. More precisely, by semantics of $\operatorname{tr}, \mathrm{r}^{\prime}=\mathrm{r}-1$, for each $i: 0 \leq i \leq a, \operatorname{val}_{i}^{\mathrm{c}^{\prime}}=\operatorname{val}_{F(i)}^{1}$, 
$\operatorname{val}_{i}^{\mathrm{c}}=\operatorname{val}_{F(i)}^{2}$, and $\mathrm{HO}_{i}^{\mathrm{c}^{\prime}}$ satisfies $\operatorname{grd}_{F(i)}$ (Note that the numbers of processes in $c$ and $c^{\prime}$ are equal).

Assume that the surjection $\operatorname{Sr}::\{1, \ldots, a\} \rightarrow\{1, \ldots, b\}$ witnesses $c \vDash \varphi$. We exhibit a constraint $\varphi^{\prime}$ that denotes $c^{\prime}$ and is generated by the predecessor computation function, i.e. $\varphi^{\prime} \in \operatorname{pre}_{\mathrm{tr}}(\varphi)$.

All generated $e^{\prime}$ capture $r^{\prime}$ if $c^{\prime} \rightarrow c$ and $c \in[\varphi \varphi]$. More precisely, $e=(r$ $\bmod |\operatorname{Tr}|)$ and $e^{\prime}=((e-1) \bmod |\operatorname{Tr}|)$ captures $r^{\prime}=r-1$. Observe that for $j: 1 \leq j \leq b$, Sr maps each $\operatorname{val}_{j}^{\varphi}$ to some $\operatorname{val}_{i}^{\mathrm{c}}$.

We choose I to be $\{(F(i), S(i)) \mid i: 1 \leq i \leq a\}$. Such I is valid according to Figure 8.4, because,

- By definition of $F$, image $(F) \subseteq\{1, \ldots, K\}$, hence, $I \subseteq\{1, \ldots, K\} \times$ $\{1, \ldots, b\}$

- Sr is total and surjective, thus, $\mathrm{I}_{\{1, \ldots, b\}}=\{1, \ldots, b\}$

Let $\operatorname{Sr}^{\prime}$ be a surjection $\operatorname{Sr}^{\prime}=\{1, \ldots, a\} \rightarrow\{1, \ldots,|\mathrm{I}|\}$ where $\operatorname{Sr}^{\prime}(i)=$ $\mathrm{H}^{-1}((F(i), \mathrm{Sr}(i)))$. Since $F$ and $\mathrm{Sr}$ are total functions, we are sure that $\operatorname{Sr}^{\prime}(i)$ is defined for all $i: 1 \leq i \leq a$.

By definition of $F$ and $\mathrm{Sr}, \operatorname{val}_{i}^{\mathrm{c}^{\prime}}=\operatorname{val}_{F(i)}^{1}=\operatorname{val}_{\mathrm{Sr}^{\prime}(i)}^{\varphi^{\prime}}$ and $\operatorname{val}_{i}^{\mathrm{c}}=\operatorname{val}_{\mathrm{Sr}(i)}^{\varphi}=$ $\operatorname{val}_{F(i)}^{2}$. Hence, each val $c_{i}^{c^{\prime}}$ is mapped to some $\operatorname{val}_{j^{\prime}}^{\varphi^{\prime}}$.

Benign faults. Since $c^{\prime} \stackrel{\operatorname{tr}}{\longrightarrow} c, g l^{\prime}\left({ }^{\#} \mathrm{~V}^{\prime}\right)$ is satisfiable in the presence of benign faults. Such faults respect the axioms $\operatorname{HAX}_{k}\left({ }^{\#} \mathrm{HO}_{k},{ }^{\#} \mathrm{~V}^{\prime}\right)=\bigwedge_{\mathrm{d} \in \mathrm{D}} 0 \leq{ }^{\#} \mathrm{ho}_{\mathrm{k}}^{\mathrm{d}} \leq$ ${ }^{\#} \mathrm{v}^{\prime}$. Assume the following solution for $\mathrm{gl}^{\prime}\left({ }^{\#} \mathrm{~V}^{\prime}\right)$ :

- for each $\mathrm{v}$ and $\mathrm{d}$, replace $\#_{\mathrm{v}^{\mathrm{d}}}$ and $\#_{\mathrm{v}^{\prime}}{ }^{\mathrm{d}}$ respectively with valuesOf $(c, v)(d)$ and valuesOf $\left(c^{\prime}, v\right)(d)$,

- for each $i$ and $\mathrm{d}$, replace ${ }^{\#} \mathrm{ho}_{\mathrm{F}(\mathrm{i})}^{\mathrm{d}}$ with $\left|\mathrm{HO}_{\mathrm{i}}^{\mathrm{d}}\right|$,

- replace $n$ with $a$.

The proposed $\varphi^{\prime}$ denotes $c^{\prime}$, because, 1) $((r-1) \bmod |\operatorname{Tr}|=(e-1)$ $\bmod |\operatorname{Tr}|$ ) (note that $\mathrm{e}=\mathrm{r} \bmod |\mathrm{Tr}|), 2)$ by definition of $F$ and $\mathrm{Sr}^{\prime}$, for all $1 \leq i \leq a, \operatorname{val}_{i}^{\mathrm{c}^{\prime}}=\operatorname{val}_{F(i)}^{1}=\operatorname{val}_{\mathrm{Sr}^{\prime}(i)}^{\varphi^{\prime}}$, and 3) according to the exhibited solution for $\mathrm{gl}^{\prime}\left({ }^{\#} \mathrm{~V}^{\prime}\right)$, replacing each occurrence of ${ }^{\#} \mathrm{v}^{\mathrm{d}}$ in the global condition $\mathrm{gl} \mathbf{l}^{\prime}$ by valuesOf $(\mathrm{c}, \mathrm{v})(\mathrm{d})$ and each occurrence of $n$ by $a$ results in a valid formula. Because $g l^{\prime}\left({ }^{\#} \mathrm{~V}^{\prime}\right)$ is an over-approximation of the exact guard that is satisfied in $c^{\prime}$, by existence of $c^{\prime}$ we are sure that $g l^{\prime}\left({ }^{\#} \mathrm{~V}^{\prime}\right)$ evaluates to true. 


$$
\begin{aligned}
& \mathrm{c}^{\prime}:\left(\mathrm{r}^{\prime},\left\langle\mathrm{p}_{1}^{\mathrm{c}^{\prime}} \ldots, \mathrm{p}_{i}^{\mathrm{c}^{\prime}}=\left(\operatorname{val}_{i}^{\mathrm{c}^{\prime}}, \mathrm{HO}_{i}^{\mathrm{c}^{\prime}}\right) \ldots, \mathrm{p}_{a}^{\mathrm{c}^{\prime}}>\right) \underset{\left(\operatorname{grd}_{F(i)}: \operatorname{val}_{F(i)}^{1} \rightarrow \operatorname{val}_{F(i)}^{2}\right)}{\mathrm{F}:\{1, \ldots, a\} \rightarrow\{1, \ldots, K\}} \mathrm{c}:\left(\mathrm{r},\left\langle\mathrm{p}_{1}^{\mathrm{c}} \ldots, \mathrm{p}_{i}^{\mathrm{c}}=\left(\operatorname{val}_{i}^{\mathrm{c}}, \mathrm{HO}_{\mathrm{i}}^{\mathrm{c}}\right) \ldots, \mathrm{p}_{a}^{\mathrm{c}}>\right)\right.\right.
\end{aligned}
$$

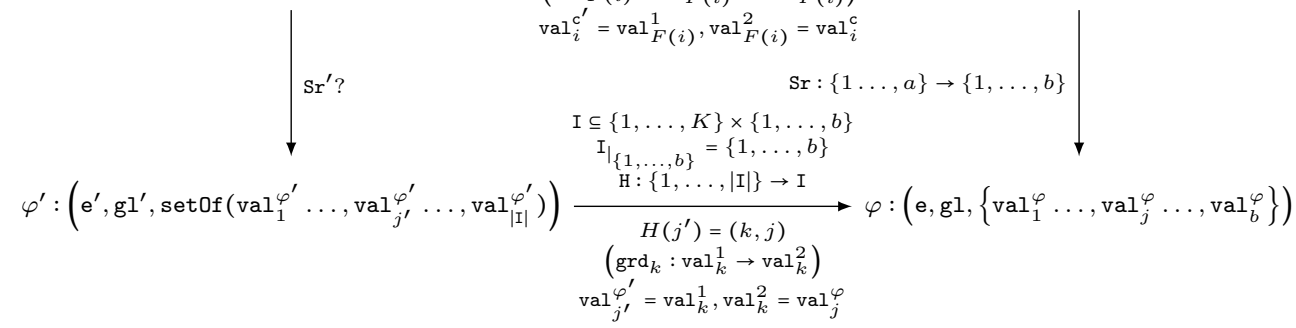

Figure 8.5: Illustration for proof of soundness of predecessor computation for HO programs. Given $c^{\prime} \rightarrow c$ and $c \vDash \varphi$, soundness boils down to showing the existence of $\varphi^{\prime} \in \operatorname{pre}_{\operatorname{tr}}(\varphi)$ for which $c^{\prime} \vDash \varphi^{\prime}$.

Corruption In the case of corruption faults, DAX is the same as benign faults, but for all $F(i)$ where $(F(i), \operatorname{Sr}(i))$ is in $\mathrm{I}, \operatorname{HAX}_{F(i)}=\bigwedge_{\mathrm{d} \in \mathrm{D}}\left(0 \leq{ }^{\#} \mathrm{ho}_{\mathrm{F}(\mathrm{i})}^{\mathrm{d}} \leq\right.$ $\left.\#{ }_{\mathrm{v}^{\prime}}{ }^{\mathrm{d}}+\alpha\right)$. By the existence of $\mathrm{c}^{\prime}$, we have that $\bigwedge_{1 \leq F(i) \leq K}\left({ }^{\#} \mathrm{~h} \mathrm{o}_{\mathrm{F}(\mathrm{i})}^{\mathrm{d}} \leq \#{ }^{\prime}{ }^{\mathrm{d}}+\alpha\right)$ for each value $d \in D$. The solution for $\mathrm{gl}^{\prime}\left({ }^{\#} \mathrm{~V}^{\prime}\right)$ in the case of corruption is similar to the benign case, plus, it needs to satisfy $\max _{\substack{\mathrm{d} \in \mathrm{D} \\ 1 \leq F(i) \leq c}}\left({ }^{*} \mathrm{ho} \mathrm{F}_{\mathrm{F}(\mathrm{i})}^{\mathrm{d}}-{ }^{*} \mathrm{v}^{\prime}{ }^{\mathrm{d}}\right) \leq \alpha$. The existence of $c^{\prime}$ ensures that this condition is satisfiable. This is because $\mathrm{gl}^{\prime}\left({ }^{\#} \mathrm{~V}^{\prime}\right)$ is an over-approximation of the exact guard that is satisfied in $\mathrm{c}^{\prime}$.

Theorem 24. The proposed procedure terminates.

Termination is obtained by the fact that at most a finite number of constraints might be generated. To see this, observe that the constraints consist of an integer capturing control location, a predicate (the global condition), and a set of local valuations of processes (the base). The number of control locations and that of the local valuations of the processes are finite. In addition, only finitely many global conditions are generated for a protocol. This is due to the fact that the number of the subsets of the guarded commands which are used for the predicate abstraction $\left(\operatorname{PrAbs}_{[\Gamma]}\right)$ is finite, and the invariants (Inv) and the strengthening invariants needed for corruption fault (e.g., see Figure 8.3) are fixed for each protocol.

\subsection{Experimental Results}

We report on the experiments with our open-source prototype tool SyncV, which is publicly available at https://gitlab.liu.se/live/syncv to verify a class 
of $\mathrm{HO}$ algorithms. The experiments were conducted on an Intel Core(TM) i7 processor running at $2.9 \mathrm{GHz}$ with $8 \mathrm{~GB}$ of memory. We conducted the experiments on several variations of the One-Third-Rule and $\mathcal{A}_{E, T}$ algorithms. These variations correspond to checking the correctness properties of agreement, validity, and irrevocability for correct and buggy versions of the considered algorithms and an unbounded number of processes. For each property, we verified a correct version and a buggy version. The buggy versions differ from the correct ones in the guards of their commands. For verification of the $\mathcal{A}_{E, T}$ algorithm, we strengthened our invariant Inv in Figure 8.4 with the invariants represented in Figure 8.3 that according to [23] are essential for the correctness of the algorithm.

For all correct versions, our tool reported that the program is safe, and for all buggy ones, it presented a valid trace that violates the considered property. Our implemented procedure does not eagerly concretize the local valuations of the processes. Instead, we concretize on demand. All benchmarks are available on the tool homepage.

Checking different correctness properties. We discussed in depth checking the agreement correctness property in sections 8.2 and 8.5. Checking the validity property is similar because it also uses a finite set of forbidden valuations to characterize the set of bad constraints. For checking irrevocability, one needs to see if a process can make a decision and revoke it later. To make such checks, we take into account a history of the changes. We do that by augmenting each process group in a constraint by a list of possible decisions as its history. This list is empty by default. A bad constraint that violates irrevocability has at least one process group with a minimum of two different values in its history.

\subsection{Conclusion and Future Work}

We have studied a subclass of fault-tolerant distributed algorithms in terms of the Heard-Of model and proposed a symbolic representation that uses cardinality constraints on multisets to model sets of configurations generated during the analysis of such programs. We have also introduced a sound procedure for checking state reachability to check various correctness properties, such as agreement, validity, and irrevocability for consensus programs in the presence of lossy or corrupt communications. We showed that the introduced procedure terminates even for an unbounded number of processes. To the best of our knowledge, this is the first fully-automatic approach to verifying Heard-Of protocols in the presence of corrupt communications. We reported on our experiments with the correct and buggy variations of the protocols. 


\begin{tabular}{|c|c|c|c|c|}
\hline program & property & safe? & result & $\operatorname{time}(\mathrm{m})$ \\
\hline simple & $\begin{array}{l}\text { agreement } \\
\text { validity } \\
\text { irrevocability }\end{array}$ & $\begin{array}{l}\checkmark \\
\checkmark \\
\checkmark\end{array}$ & $\begin{array}{l}\text { safe } \\
\text { safe } \\
\text { safe }\end{array}$ & $\begin{array}{c}2 \\
\sim 0 \\
1\end{array}$ \\
\hline \multirow{3}{*}{$\frac{1}{3}$-rule } & agreement & $\begin{array}{l}\checkmark \\
x\end{array}$ & $\begin{array}{l}\text { safe } \\
\text { trace }\end{array}$ & $\begin{array}{l}19 \\
\sim 0\end{array}$ \\
\hline & validity & $\begin{array}{l}\sqrt{ } \\
x\end{array}$ & $\begin{array}{c}\text { safe } \\
\text { trace }\end{array}$ & $\begin{array}{c}2 \\
\sim 0\end{array}$ \\
\hline & irrevocability & $\begin{array}{l}\sqrt{ } \\
x\end{array}$ & $\begin{array}{l}\text { safe } \\
\text { trace }\end{array}$ & $\begin{array}{c}7 \\
\sim 0\end{array}$ \\
\hline \multirow{3}{*}{$\mathcal{A}_{E, T}$} & agreement & $\begin{array}{l}\sqrt{ } \\
x\end{array}$ & $\begin{array}{l}\text { safe } \\
\text { trace }\end{array}$ & $\begin{array}{c}54 \\
1\end{array}$ \\
\hline & validity & $\begin{array}{l}\checkmark \\
x\end{array}$ & $\begin{array}{l}\text { safe } \\
\text { trace }\end{array}$ & $\begin{array}{c}5 \\
\sim 0\end{array}$ \\
\hline & irrevocability & $\begin{array}{l}\checkmark \\
x\end{array}$ & $\begin{array}{l}\text { safe } \\
\text { trace }\end{array}$ & $\begin{array}{l}21 \\
\sim 0\end{array}$ \\
\hline
\end{tabular}

Table 8.1: The results of checking agreement, validity, and irrevocability for some HO protocols with SyncV. For each algorithm, a correct and a buggy version were tested by the tool. The buggy versions differ from the correct ones by the guards of their commands. For all the correct versions our tool reported that the program is safe and for all the buggy ones, it presented a valid trace violating the considered property.

Future Work. Future work can consider improving the scalability of the tool and also extending the presented technique to more general models and more sophisticated faults such as Byzantine faults. Also, the current technique assumes symmetric processes in the sense that all of them execute the same operation in each round. One could extend the model to non-symmetric processes as in the Heard-Of examples having coordinators, for instance in CoordUniform Voting and LastVoting algorithms in [30], or the Phase King and Phase Queen algorithms introduced in [20] in which a King or Queen is distinguished in each round, or the reliable broadcast algorithm in [119]. It would also be interesting to combine the approach with abstract interpretation for loops to capture the distributed algorithms in which the number of iterations is crucial for the correctness of the algorithm, for example, the FloodMin algorithm in [31]. Moreover, identifying conditions for completeness of the approach, automatic refinement of the over-approximation, and combination with richer theories are interesting directions for future work. 



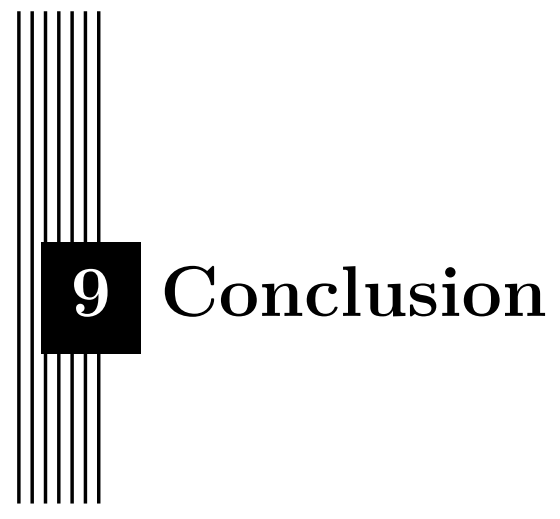

In this thesis, we have extended model checking of parameterized systems to several classes of infinite-state systems, mostly parameterized systems. We have provided an open-source prototype tool for most of the techniques that we have developed and have presented our experimental results on several benchmarks.

First, we have proposed a gap-order-based reachability analysis for bounded phaser programs. We have shown our analysis to be exact and guaranteed to terminate when checking runtime, race, and assertion errors. We have established the undecidability of deadlock verification and explained how to turn our analysis into a sound over-approximation.

Second, we have studied parameterized reachability problems for phaser programs. We have proposed an exact verification procedure for non-atomic programs when only distinguished tasks such as main create phasers. The procedure can be used for answering both control and plain reachability problems. The procedure is guaranteed to terminate, even for programs that may generate arbitrarily many tasks but finitely many phasers, when checking control (e.g., assertions) reachability or when checking plain (e.g., deadlocks) reachability with bounded gaps. These results were obtained using a non-trivial symbolic representation for which termination had required showing an $\exists \leq_{\forall}$ preorder on multisets on gaps on natural numbers to be a $\mathcal{W} \mathcal{Q} \mathcal{O}$. We believe that our general decidability results are useful to reason about synchronization constructs other than phasers. For instance, a traditional static barrier can be captured with one phaser and with bounded gaps (in fact, one). Sim- 
ilarly, one phaser with one producer and arbitrarily many consumers can be used to capture futures where a "get" instruction can be modeled with a wait. We believe that our negative results can also be used. For instance, atomic instructions can be modeled using a testAndSet operation and may result in the undecidability of the reachability problem. An interesting direction for future work could be trying to find decidable subclasses of the phaser programs with parameterized phasers. One could also consider using the technique in combination with predicate abstraction and abstract interpretation in order to analyze actual source code. Moreover, one could investigate the applicability of the presented techniques for the verification of similar synchronization constructs.

Third, we have presented a technique, predicated constrained monotonic abstraction, for the automated verification of concurrent programs whose correctness depends on the synchronization between arbitrarily many processes, for example, utilizing barriers implemented using integer counters and tests. We have introduced counting predicates that mix counters referring to the number of processes satisfying some given properties and variables that are directly manipulated by the concurrent processes. We have used counting predicates in a framework that combines predicate, counter, and constrained monotonic abstraction. Our prototype implementation gave encouraging results and managed to automatically establish or refute program assertions and deadlock freedom. Future work could consider adapting PACMAN to the synchronous protocols introduced in Chapter 8 in order to perform predicate abstraction on them and count the processes that satisfy different predicates.

Fourth, we have introduced and discussed three different ways of localizing constrained monotonic abstraction in systems with infinite control. For this, we have targeted reachability problems for (possibly non-well-structured) counter machines obtained as abstractions of concurrent programs. Our new techniques allow us to avoid trashing the state-space being explored before encountering the false positives that necessitate the introduction of new refinement predicates. This allowed us to consistently improve on the existing eager exploration, both in forward and in backward explorations. Possible future works could consider combining forward and backward approximations, using the pivot configuration to make it possible to choose interpolants that are easier to generalize, assessing the feasibility of combination with partialorder techniques, or performing the lazy explorations in parallel. The latter is more suitable for the the lazy variants since they do not delete the explored state-space upon finding new refinement predicates.

Finally, we have studied a subclass of fault-tolerant distributed algorithms in terms of the Heard-Of model and proposed a symbolic representation using cardinality constraints on multisets to model sets of configurations generated during the analysis of such programs. We have also introduced a sound procedure for checking state reachability to check various correctness properties for consensus programs such as agreement, validity, and irrevocability in the 
presence of lossy or corrupt communications. We showed that the introduced procedure terminates even for an unbounded number of processes. To the best of our knowledge, this is the first fully-automatic approach to verifying Heard-Of protocols in the presence of corrupt communication. We reported on preliminary experiments with correct and buggy variations of the protocols. Future work can consider extending the presented technique to more general models such as $[22,21]$ and more sophisticated faults such as Byzantine faults. Also, one could extend the model to non-symmetric processes as in the Heard-Of protocols that have coordinators. It would also be interesting to capture distributed algorithms in which the number of iterations is crucial for the correctness of the algorithm. Moreover, identifying conditions for completeness of the approach, and automatically refining the over-approximation are interesting directions for future work. 



\section{Bibliography}

[1] Parosh Aziz Abdulla, Aurore Annichini, Saddek Bensalem, Ahmed Bouajjani, Peter Habermehl, and Yassine Lakhnech. "Verification of Infinite-State Systems by Combining Abstraction and Reachability Analysis." In: Proceedings of the 11th International Conference on Computer Aided Verification, CAV 1999, Trento, Italy, July 1999. Vol. 1633. Lecture Notes in Computer Science. Springer, 1999, pp. 146159 .

[2] Parosh Aziz Abdulla, Karlis Cerans, Bengt Jonsson, and Yih-Kuen Tsay. "General Decidability Theorems for Infinite-State Systems." In: Proceedings of the 11th Annual IEEE Symposium on Logic in Computer Science, New Brunswick, New Jersey, USA, July 1996. IEEE Computer Society, 1996, pp. 313-321.

[3] Parosh Aziz Abdulla, Yu-Fang Chen, Giorgio Delzanno, Frédéric Haziza, Chih-Duo Hong, and Ahmed Rezine. "Constrained Monotonic Abstraction: A CEGAR for Parameterized Verification." In: Proceedings of the 21th International Conference on Concurrency Theory, CONCUR 2010, Paris, France, August 2010, vol. 6269. Lecture Notes in Computer Science. Springer, 2010, pp. 86-101.

[4] Parosh Aziz Abdulla, Giorgio Delzanno, Noomene Ben Henda, and Ahmed Rezine. "Regular Model Checking Without Transducers (On Efficient Verification of Parameterized Systems)." In: Proceedings of the 13th International Conference on Tools and Algorithms for the Construction and Analysis of Systems, TACAS 2007, Braga, Portugal, 
March 200\%. Vol. 4424. Lecture Notes in Computer Science. Springer, 2007, pp. 721-736.

[5] Parosh Aziz Abdulla, Giorgio Delzanno, and Ahmed Rezine. "Parameterized Verification of Infinite-State Processes with Global Conditions." In: Proceedings of the 19th International Conference on Computer Aided Verification, CAV 200\%, Berlin, Germany, July 200\%. Vol. 4590. Lecture Notes in Computer Science. Springer, 2007, pp. 145-157.

[6] Parosh Aziz Abdulla, Frédéric Haziza, and Lukás Holík. "All for the Price of Few." In: Proceedings of the 14th International Conference on Verification, Model Checking, and Abstract Interpretation, VMCAI 2013, Rome, Italy, January 2013. Vol. 7737. Lecture Notes in Computer Science. Springer, 2013, pp. 476-495.

[7] Parosh Aziz Abdulla, Frédéric Haziza, and Lukás Holík. "Block Me If You Can! - Context-Sensitive Parameterized Verification." In: Proceedings of the 21st International Symposium on Static Analysis, SAS 2014, Munich, Germany, September 2014. Vol. 8723. Lecture Notes in Computer Science. Springer, 2014, pp. 1-17.

[8] Parosh Aziz Abdulla, Noomene Ben Henda, Giorgio Delzanno, and Ahmed Rezine. "Handling Parameterized Systems with Non-atomic Global Conditions." In: Proceedings of the 9th International Conference on Verification, Model Checking, and Abstract Interpretation, VMCAI 2008, San Francisco, USA, January 2008. Vol. 4905. Lecture Notes in Computer Science. Springer, 2008, pp. 22-36.

[9] Francesco Alberti, Silvio Ghilardi, and Elena Pagani. "Cardinality constraints for arrays (decidability results and applications)." In: Formal Methods Syst. Des. 51.3 (2017), pp. 545-574.

[10] Peter Anderson, Brandon Chase, and Eric Mercer. "JPF verification of habanero Java programs." In: ACM SIGSOFT Softw. Eng. Notes 39.1 (2014), pp. 1-7.

[11] Krzysztof R. Apt and Dexter Kozen. "Limits for Automatic Verification of Finite-State Concurrent Systems." In: Inf. Process. Lett. 22.6 (1986), pp. 307-309.

[12] Tamarah Arons, Amir Pnueli, Sitvanit Ruah, Jiazhao Xu, and Lenore D. Zuck. "Parameterized Verification with Automatically Computed Inductive Assertions." In: Proceedings of the 13th International Conference on Computer Aided Verification, CAV 2001, Paris, France, July 2001. Vol. 2102. Lecture Notes in Computer Science. Springer, 2001, pp. 221-234.

[13] Christel Baier and Joost-Pieter Katoen. Principles of model checking. MIT press, 2008. 
[14] Thomas Ball, Rupak Majumdar, Todd D. Millstein, and Sriram K. Rajamani. "Automatic Predicate Abstraction of C Programs." In: Proceedings of the 2001 ACM SIGPLAN Conference on Programming Language Design and Implementation (PLDI), Snowbird, Utah, USA, June 2001. ACM, 2001, pp. 203-213.

[15] Thomas Ball and Sriram K. Rajamani. "The SLAM project: debugging system software via static analysis." In: Proceedings Record of the 29th SIGPLAN-SIGACT Symposium on Principles of Programming Languages, POPL 2002, Portland, OR, USA, January 2002. ACM, 2002, pp. 1-3.

[16] Kshitij Bansal, Eric Koskinen, Thomas Wies, and Damien Zufferey. "Structural Counter Abstraction." In: Proceedings of the 19th International Conference on Tools and Algorithms for the Construction and Analysis of Systems, TACAS 2013, Rome, Italy, March 2013. Vol. 7795. Lecture Notes in Computer Science. Springer, 2013, pp. 62-77.

[17] Sébastien Bardin, Alain Finkel, and Jérôme Leroux. "FASTer Acceleration of Counter Automata in Practice." In: Proceedings of the 10th International Conference on Tools and Algorithms for the Construction and Analysis of Systems, TACAS 2004, Barcelona, Spain, March 2004. Vol. 2988. Lecture Notes in Computer Science. Springer, 2004, pp. 576-590.

[18] Sébastien Bardin, Jérôme Leroux, and Gérald Point. "FAST Extended Release." In: Proceedings of the 18th International Conference on Computer Aided Verification, CAV 2006, Seattle, WA, USA, August 2006. Vol. 4144. Lecture Notes in Computer Science. Springer, 2006, pp. 6366.

[19] Gérard Basler, Matthew Hague, Daniel Kroening, C.-H. Luke Ong, Thomas Wahl, and Haoxian Zhao. "Boom: Taking Boolean Program Model Checking One Step Further." In: Proceedings of the 16th International Conference on Tools and Algorithms for the Construction and Analysis of Systems, TACAS 2010, Paphos, Cyprus, March 2010. Vol. 6015. Lecture Notes in Computer Science. Springer, 2010, pp. 145149.

[20] Piotr Berman, Juan A. Garay, and Kenneth J. Perry. "Optimal Early Stopping in Distributed Consensus (Extended Abstract)." In: Proceedings of the 6th International Workshop on Distributed Algorithms, WDAG 1992, Haifa, Israel, November 1992. Vol. 647. Lecture Notes in Computer Science. Springer, 1992, pp. 221-237.

[21] Nathalie Bertrand, Igor Konnov, Marijana Lazic, and Josef Widder. "Verification of Randomized Consensus Algorithms Under RoundRigid Adversaries." In: Proceeding of the 30th International Conference on Concurrency Theory, CONCUR 2019, August 2019, Amster- 
dam, the Netherlands. Ed. by Wan J. Fokkink and Rob van Glabbeek. Schloss Dagstuhl - Leibniz-Zentrum für Informatik, 2019, 33:1-33:15.

[22] Nathalie Bertrand, Marijana Lazic, and Josef Widder. "A Reduction Theorem for Randomized Distributed Algorithms Under Weak Adversaries." In: Proceedings of the 22nd International Conference on Verification, Model Checking, and Abstract Interpretation, VMCAI 2021, Copenhagen, Denmark, January 2021. Ed. by Fritz Henglein, Sharon Shoham, and Yakir Vizel. Springer, 2021, pp. 219-239.

[23] Martin Biely, Josef Widder, Bernadette Charron-Bost, Antoine Gaillard, Martin Hutle, and André Schiper. "Tolerating corrupted communication." In: Proceedings of the 26th Annual ACM Symposium on Principles of Distributed Computing, PODC 2007, Portland, Oregon, USA, August 200\%. ACM, 2007, pp. 244-253.

[24] Frank S. de Boer, Dave Clarke, and Einar Broch Johnsen. "A Complete Guide to the Future." In: Proceedings of the 16th European Symposium on Programming Languages and Systems, ESOP 2007, Braga, Portugal, March 200\%. Vol. 4421. Lecture Notes in Computer Science. Springer, 2007, pp. 316-330.

[25] Rémi Bonnet, Alain Finkel, Jérôme Leroux, and Marc Zeitoun. "Model Checking Vector Addition Systems with one zero-test." In: Log. Methods Comput. Sci. 8.2 (2012).

[26] Ahmed Bouajjani, Marius Bozga, Peter Habermehl, Radu Iosif, Pierre Moro, and Tomás Vojnar. "Programs with Lists Are Counter Automata." In: Proceedings of the 18th International Conference on Computer Aided Verification, CAV 2006, Seattle, WA, USA, August 2006. Vol. 4144. Lecture Notes in Computer Science. Springer, 2006, pp. 517 531.

[27] Laura Bozzelli and Sophie Pinchinat. "Verification of gap-order constraint abstractions of counter systems." In: Theoretical Computer Science 523 (2014), pp. 1-36.

[28] Vincent Cavé, Jisheng Zhao, Jun Shirako, and Vivek Sarkar. "Habanero-Java: the new adventures of old X10." In: Proceedings of the 9th International Conference on Principles and Practice of Programming in Java, PPPJ 2011, Kongens Lyngby, Denmark, August 2011. ACM, 2011, pp. 51-61.

[29] Philippe Charles, Christian Grothoff, Vijay A. Saraswat, Christopher Donawa, Allan Kielstra, Kemal Ebcioglu, Christoph von Praun, and Vivek Sarkar. "X10: an object-oriented approach to non-uniform cluster computing." In: (2005), pp. 519-538. 
[30] Bernadette Charron-Bost and André Schiper. "The Heard-Of model: computing in distributed systems with benign faults." In: Distributed Comput. 22.1 (2009), pp. 49-71.

[31] Soma Chaudhuri, Maurice Herlihy, Nancy A. Lynch, and Mark R. Tuttle. "Tight bounds for $k$-set agreement." In: J. ACM 47.5 (2000), pp. $912-943$.

[32] Edmund M. Clarke and E. Allen Emerson. "Design and Synthesis of Synchronization Skeletons Using Branching-Time Temporal Logic." In: Logics of Programs, Workshop, Yorktown Heights, New York, USA, 1981. Vol. 131. Lecture Notes in Computer Science. Springer, 1981, pp. $52-71$.

[33] Edmund M. Clarke, Orna Grumberg, and Michael C. Browne. "Reasoning About Networks With Many Identical Finite-State Processes." In: Proceedings of the 5th Annual ACM Symposium on Principles of Distributed Computing, Calgary, Alberta, Canada, August 1986. ACM, 1986, pp. 240-248.

[34] Edmund M. Clarke, Orna Grumberg, and Somesh Jha. "Verifying parameterized networks using abstraction and regular languages." In: International Conference on Concurrency Theory. Springer. 1995, pp. 395-407.

[35] Edmund M. Clarke, Orna Grumberg, Somesh Jha, Yuan Lu, and Helmut Veith. "Counterexample-Guided Abstraction Refinement." In: Proceedings of the 12th International Conference on Computer Aided Verification, CAV 2000, Chicago, IL, USA, July 2000. Vol. 1855. Lecture Notes in Computer Science. Springer, 2000, pp. 154-169.

[36] Edmund M. Clarke, Orna Grumberg, and David E. Long. "Model checking and abstraction." In: ACM Transactions on Programming Languages and Systems (TOPLAS) 16.5 (1994), pp. 1512-1542.

[37] Edmund M. Clarke, Orna Grumberg, and Doron A. Peled. Model checking. MIT Press, 2001.

[38] Edmund M. Clarke, Daniel Kroening, Natasha Sharygina, and Karen Yorav. "SATABS: SAT-Based Predicate Abstraction for ANSI-C." In: Tools and Algorithms for the Construction and Analysis of Systems, TACAS 2005, Edinburgh, UK, April 2005, vol. 3440. Lecture Notes in Computer Science. Springer, 2005, pp. 570-574.

[39] Edmund M. Clarke, Muralidhar Talupur, and Helmut Veith. "Environment Abstraction for Parameterized Verification." In: Proceedings of the 7th International Conference on Verification, Model Checking, and Abstract Interpretation, VMCAI 2006, Charleston, SC, USA, January 2006. Vol. 3855. Lecture Notes in Computer Science. Springer, 2006, pp. $126-141$. 
[40] Tiago Cogumbreiro, Raymond Hu, Francisco Martins, and Nobuko Yoshida. "Dynamic deadlock verification for general barrier synchronisation." In: Proceedings of the 20th ACM SIGPLAN Symposium on Principles and Practice of Parallel Programming, PPoPP 2015, San Francisco, CA, USA, February 2015. ACM, 2015, pp. 150-160.

[41] Tiago Cogumbreiro, Jun Shirako, and Vivek Sarkar. "Formalization of Habanero phasers using Coq." In: J. Log. Algebraic Methods Program. 90 (2017), pp. 50-60.

[42] Patrick Cousot. "Abstract interpretation." In: ACM Computing Surveys (CSUR) 28.2 (1996), pp. 324-328.

[43] Patrick Cousot and Radhia Cousot. "Abstract interpretation frameworks." In: Journal of logic and computation 2.4 (1992), pp. 511-547.

[44] Patrick Cousot and Radhia Cousot. "Abstract Interpretation: A Unified Lattice Model for Static Analysis of Programs by Construction or Approximation of Fixpoints." In: Proceedings of the 4th ACM Symposium on Principles of Programming Languages, Los Angeles, California, USA, January 197\%. ACM, 1977, pp. 238-252.

[45] John Davis, Christopher Hylands, Jörn Janneck, Edward A. Lee, Jie Liu, Xiaojun Liu, Steve Neuendorffer, Sonia Sachs, Mary Stewart, Kees Vissers, Paul Whitaker, and Yuhong Xiong. Overview of the Ptolemy project. Tech. rep. ERL Technical Report UCB/ERL, 1999.

[46] Henri Debrat and Stephan Merz. "Verifying Fault-Tolerant Distributed Algorithms in the Heard-Of Model." In: Archive of Formal Proofs (2012). URL: https : //www . isa-afp .org/entries/Heard\%5C_Of . shtml.

[47] Giorgio Delzanno. "Automatic Verification of Parameterized Cache Coherence Protocols." In: Proceedings of the 12th International Conference on Computer Aided Verification, CAV 2000, Chicago, IL, USA, July 2000. Vol. 1855. Lecture Notes in Computer Science. Springer, 2000, pp. 53-68.

[48] L. E. Dickson. "Finiteness of the Odd Perfect and Primitive Abundant Numbers with $n$ Distinct Prime Factors." In: Amer. J. Math. 35 (1913), pp. 413-422.

[49] Edsger W. Dijkstra et al. "Notes on structured programming." In: (1970).

[50] Alastair F. Donaldson, Alexander Kaiser, Daniel Kroening, Michael Tautschnig, and Thomas Wahl. "Counterexample-guided abstraction refinement for symmetric concurrent programs." In: Formal Methods in System Design 41.1 (2012). 
[51] Alastair F. Donaldson, Alexander Kaiser, Daniel Kroening, and Thomas Wahl. "Symmetry-Aware Predicate Abstraction for SharedVariable Concurrent Programs." In: Proceedings of the 23rd International Conference on Computer Aided Verification, CAV 2011, Snowbird, UT, USA, July 2011. Vol. 6806. Lecture Notes in Computer Science. Springer, 2011, pp. 356-371.

[52] Allen Downey. The Little Book of SEMAPHORES (2nd Edition): The Ins and Outs of Concurrency Control and Common Mistakes. Createspace Ind. Pub, 2009. ISBN: 9781441418685. URL: http://books . google.se/books?id=2J2eQQAACAAJ.

[53] Cezara Dragoi, Thomas A. Henzinger, and Damien Zufferey. "PSync: a partially synchronous language for fault-tolerant distributed algorithms." In: Proceedings of the 43rd Annual ACM SIGPLAN-SIGACT Symposium on Principles of Programming Languages, POPL 2016, St. Petersburg, FL, USA, January 2016. ACM, 2016, pp. 400-415.

[54] Catherine Dufourd, Alain Finkel, and Philippe Schnoebelen. "Reset Nets Between Decidability and Undecidability." In: Proceedings of the 25th International Colloquium on Automata, Languages and Programming, ICALP 1998, Aalborg, Denmark, July 1998. Vol. 1443. Lecture Notes in Computer Science. Springer, 1998, pp. 103-115.

[55] E. Allen Emerson and Vineet Kahlon. "Reducing Model Checking of the Many to the Few." In: Proceedings of the 17th International Conference on Automated Deduction, CADE-17, Pittsburgh, PA, USA, June 2000. Vol. 1831. Lecture Notes in Computer Science. Springer, 2000, pp. 236-254.

[56] E. Allen Emerson and Kedar S. Namjoshi. "Automatic Verification of Parameterized Synchronous Systems (Extended Abstract)." In: Proceedings of the 8th International Conference on Computer Aided Verification CAV, New Brunswick, NJ, USA, July 1996. Vol. 1102. Lecture Notes in Computer Science. Springer, 1996, pp. 87-98.

[57] Javier Esparza, Ruslán Ledesma-Garza, Rupak Majumdar, Philipp J. Meyer, and Filip Niksic. "An SMT-Based Approach to Coverability Analysis." In: Proceedings of the 26th International Conference on Computer Aided Verification, CAV 2014, Vienna, Austria, July 2014. Vol. 8559. Lecture Notes in Computer Science. Springer, 2014, pp. 603619.

[58] Azadeh Farzan, Zachary Kincaid, and Andreas Podelski. "Proofs that count." In: Proceedings of the 41st Annual ACM SIGPLAN-SIGACT Symposium on Principles of Programming Languages, POPL 2014, San Diego, CA, USA, January 2014. ACM, 2014, pp. 151-164. 
[59] Alain Finkel and Philippe Schnoebelen. "Well-structured transition systems everywhere!" In: Theor. Comput. Sci. 256.1-2 (2001), pp. 6392.

[60] Cormac Flanagan and Shaz Qadeer. "Thread-Modular Model Checking." In: Proceedings of the 10th International SPIN Workshop on Model Checking Software, Portland, OR, USA, May 2003. Vol. 2648. Lecture Notes in Computer Science. Springer, 2003, pp. 213-224.

[61] Zeinab Ganjei, Ahmed Rezine, Petru Eles, and Zebo Peng. "Abstracting and Counting Synchronizing Processes." In: Proceedings of the 16th International Conference on Verification, Model Checking, and Abstract Interpretation, VMCAI 2015, Mumbai, India, January 2015. Vol. 8931. Lecture Notes in Computer Science. Springer, 2015, pp. 227 244.

[62] Zeinab Ganjei, Ahmed Rezine, Petru Eles, and Zebo Peng. "Safety verification of phaser programs." In: Proceedings of the 15th International Conference on Formal Methods in Computer Aided Design, FMCAD 2017, Vienna, Austria, October 2017. IEEE, 2017, pp. 68-75.

[63] Gilles Geeraerts, Jean-François Raskin, and Laurent Van Begin. "Expand, Enlarge and Check... Made Efficient." In: Proceedings of the 17th International Conference on Computer Aided Verification, CAV 2005, Edinburgh, Scotland, UK, July 2005. Vol. 3576. Lecture Notes in Computer Science. Springer, 2005, pp. 394-407.

[64] Steven M. German and A. Prasad Sistla. "Reasonings about Systems with Many Processes." In: J. ACM 39.3 (1992), pp. 675-735.

[65] Silvio Ghilardi and Silvio Ranise. "MCMT: A Model Checker Modulo Theories." In: Proceedings of the 5th International Joint Conference on Automated Reasoning, IJCAR 2010, Edinburgh, UK, July 2010. Vol. 6173. Lecture Notes in Computer Science. Springer, 2010, pp. 2229.

[66] Klaus von Gleissenthall, Nikolaj Bjørner, and Andrey Rybalchenko. "Cardinalities and universal quantifiers for verifying parameterized systems." In: Proceedings of the 37th ACM SIGPLAN Conference on Programming Language Design and Implementation, PLDI 2016, Santa Barbara, CA, USA, June 2016. ACM, 2016, pp. 599-613.

[67] Susanne Graf and Hassen Saïdi. "Construction of Abstract State Graphs with PVS." In: Proceedings of the 9th International Conference on Computer Aided Verification, CAV 1997, Haifa, Israel, June 1997. Vol. 1254. Lecture Notes in Computer Science. Springer, 1997, pp. $72-83$. 
[68] Klaus Havelund and Thomas Pressburger. "Model Checking JAVA Programs using JAVA PathFinder." In: Int. J. Softw. Tools Technol. Transf. 2.4 (2000), pp. 366-381.

[69] Chris Hawblitzel, Jon Howell, Manos Kapritsos, Jacob R. Lorch, Bryan Parno, Michael L. Roberts, Srinath T. V. Setty, and Brian Zill. "IronFleet: proving practical distributed systems correct." In: Proceedings of the 25th Symposium on Operating Systems Principles, SOSP 2015, Monterey, CA, USA, October 2015. ACM, 2015, pp. 1-17.

[70] Thomas A. Henzinger, Ranjit Jhala, Rupak Majumdar, and Shaz Qadeer. "Thread-Modular Abstraction Refinement." In: Proceedings of the 15th International Conference on Computer Aided Verification, CAV 2003, Boulder, CO, USA, July 2003. Vol. 2725. Lecture Notes in Computer Science. Springer, 2003, pp. 262-274.

[71] Thomas A. Henzinger, Ranjit Jhala, Rupak Majumdar, and Grégoire Sutre. "Lazy abstraction." In: Proceedings of the 29th SIGPLANSIGACT Symposium on Principles of Programming Languages, POPL 2002, Portland, OR, USA, January 2002. ACM, 2002, pp. 58-70.

[72] Graham Higman. "Ordering by divisibility in abstract algebras." In: Proceedings of the London Mathematical Society 3.1 (1952), pp. 326336.

[73] Charles A. R. Hoare. "An Axiomatic Basis for Computer Programming." In: Commun. ACM 12.10 (1969), pp. 576-580.

[74] Charles A. R. Hoare. "Proof of a Program: FIND." In: Commun. ACM 14.1 (1971), pp. 39-45.

[75] C. Norris Ip and David L. Dill. "Verifying Systems with Replicated Components in Murphi." In: Proceedings of the 8th International Conference on Computer Aided Verification, CAV 1996, New Brunswick, NJ, USA, July 1996. Vol. 1102. Lecture Notes in Computer Science. Springer, 1996, pp. 147-158.

[76] Petr Jancar. "A Note on Well Quasi-Orderings for Powersets." In: Inf. Process. Lett. 72.5-6 (1999), pp. 155-160.

[77] Mauro Jaskelioff and Stephan Merz. "Proving the Correctness of Disk Paxos." In: Arch. Formal Proofs 2005 (2005).

[78] Ranjit Jhala and Kenneth L. McMillan. "A Practical and Complete Approach to Predicate Refinement." In: Proceedings of the 12th International on Tools and Algorithms for the Construction and Analysis of Systems, TACAS 2006, Vienna, Austria, March 2006. Vol. 3920. Lecture Notes in Computer Science. Springer, 2006, pp. 459-473. 
[79] Annu John, Igor Konnov, Ulrich Schmid, Helmut Veith, and Josef Widder. "Parameterized model checking of fault-tolerant distributed algorithms by abstraction." In: Proceedings of the Formal Methods in Computer-Aided Design, FMCAD 2013, Portland, OR, USA, October 20-23, 2013. IEEE, 2013, pp. 201-209.

[80] Vineet Kahlon, Franjo Ivancic, and Aarti Gupta. "Reasoning About Threads Communicating via Locks." In: Proceedings of the 17th International Conference on Computer Aided Verification, CAV 2005, Edinburgh, Scotland, UK, July 2005. Vol. 3576. Lecture Notes in Computer Science. Springer, 2005, pp. 505-518.

[81] Alexander Kaiser, Daniel Kroening, and Thomas Wahl. "Dynamic Cutoff Detection in Parameterized Concurrent Programs." In: Proceedings of the 22nd International Conference on Computer Aided Verification, $C A V$ 2010, Edinburgh, UK, July 2010. Vol. 6174. Lecture Notes in Computer Science. Springer, 2010, pp. 645-659.

[82] Alexander Kaiser, Daniel Kroening, and Thomas Wahl. "Efficient Coverability Analysis by Proof Minimization." In: Proceedings of the 23rd International Conference on Concurrency Theory, CONCUR 2012, Newcastle upon Tyne, UK, September 2012. Vol. 7454. Lecture Notes in Computer Science. Springer, 2012, pp. 500-515.

[83] Alexander Kaiser, Daniel Kroening, and Thomas Wahl. "Lost in Abstraction: Monotonicity in Multi-threaded Programs." In: Proceedings of the 25th International Conference on Concurrency Theory, CONCUR 2014, Rome, Italy, September 2014. Vol. 8704. Lecture Notes in Computer Science. Springer, 2014, pp. 141-155.

[84] Yonit Kesten, Amir Pnueli, Elad Shahar, and Lenore D. Zuck. "Network Invariants in Action." In: Proceedings of the 13th International Conference on Concurrency Theory, CONCUR 2002, Brno, Czech Republic, August 2002, vol. 2421. Lecture Notes in Computer Science. Springer, 2002, pp. 101-115.

[85] Johannes Kloos, Rupak Majumdar, Filip Niksic, and Ruzica Piskac. "Incremental, Inductive Coverability." In: Proceedings of the 25th International Conference on Computer Aided Verification, CAV 2013, Saint Petersburg, Russia, July 2013. Vol. 8044. Lecture Notes in Computer Science. Springer, 2013, pp. 158-173.

[86] Igor V. Konnov, Helmut Veith, and Josef Widder. "On the completeness of bounded model checking for threshold-based distributed algorithms: Reachability." In: Inf. Comput. 252 (2017), pp. 95-109. 
[87] Igor V. Konnov, Helmut Veith, and Josef Widder. "SMT and POR Beat Counter Abstraction: Parameterized Model Checking of ThresholdBased Distributed Algorithms." In: Proceedings of the 27th International Conference on Computer Aided Verification, CAV 2015, San Francisco, CA, USA, July 2015. Vol. 9206. Lecture Notes in Computer Science. Springer, 2015, pp. 85-102.

[88] Igor V. Konnov, Helmut Veith, and Josef Widder. "What You Always Wanted to Know About Model Checking of Fault-Tolerant Distributed Algorithms." In: 10th International Andrei Ershov Informatics Conference on Perspectives of System Informatics, PSI 2015, Russia, August 2015. Vol. 9609. Lecture Notes in Computer Science. Springer, 2015, pp. 6-21.

[89] Saul A Kripke. "A completeness theorem in modal logic." In: The journal of symbolic logic 24.1 (1959), pp. 1-14.

[90] Viktor Kuncak, Huu Hai Nguyen, and Martin C. Rinard. "An Algorithm for Deciding BAPA: Boolean Algebra with Presburger Arithmetic." In: Proceedings of the 20th International Conference on Automated Deduction, CADE-20, Tallinn, Estonia, July 2005. Vol. 3632. Lecture Notes in Computer Science. Springer, 2005, pp. 260-277.

[91] Shuvendu K. Lahiri and Madanlal Musuvathi. "An Efficient Decision Procedure for UTVPI Constraints." In: Proceedings of the 5th International Workshop on Frontiers of Combining Systems, FroCoS 2005, Vienna, Austria, September 2005. Vol. 3717. Lecture Notes in Computer Science. Springer, 2005, pp. 168-183.

[92] Leslie Lamport. Specifying Systems, The TLA+Language and Tools for Hardware and Software Engineers. Addison-Wesley, 2002.

[93] Duy-Khanh Le, Wei-Ngan Chin, and Yong Meng Teo. "Verification of the Static and Dynamic Barrier Synchronization Using Bounded Permissions." In: Proceedings of the 15th International Conference on Formal Methods and Software Engineering, 1ICFEM 2013, Queenstown, New Zealand, October 2013. Vol. 8144. Lecture Notes in Computer Science. Springer, 2013, pp. 231-248.

[94] Edward A. Lee. "The Problem with Threads." In: Computer 39.5 (2006), pp. 33-42.

[95] Herbert Lin. "The development of software for ballistic-missile defense." In: Scientific American 253.6 (1985), pp. 46-53.

[96] Peizun Liu and Thomas Wahl. "Infinite-state backward exploration of Boolean broadcast programs." In: Proceedings of the Formal Methods in Computer-Aided Design, FMCAD 2014, Lausanne, Switzerland, October 2014. IEEE, 2014, pp. 155-162. 
[97] Boris D. Lubachevsky. "An Approach to Automating the Verification of Compact Parallel Coordination Programs I." In: Acta Informatica 21 (1984), pp. 125-169.

[98] Alberto Marcone. "Fine Analysis of the Quasi-Orderings on the Power Set." In: Order 18.4 (2001), pp. 339-347.

[99] Ognjen Maric, Christoph Sprenger, and David A. Basin. "Cutoff Bounds for Consensus Algorithms." In: Proceedings of the 29th International Conference on Computer Aided Verification, CAV 2017, Heidelberg, Germany, July 2017. Vol. 10427. Lecture Notes in Computer Science. Springer, 2017, pp. 217-237.

[100] Richard Mayr and Patrick Totzke. "Branching-Time Model Checking Gap-Order Constraint Systems." In: Fundam. Inform. 143.3-4 (2016), pp. 339-353.

[101] Kenneth L. McMillan. "An Interpolating Theorem Prover." In: Theoretical Computer Science 345.1 (2005), pp. 101-121.

[102] Kenneth L. McMillan. "Lazy Abstraction with Interpolants." In: Proceedings of the 18th International Conference on Computer Aided Verification, CAV 2006, Seattle, WA, USA, August 2006. Vol. 4144. Lecture Notes in Computer Science. Springer, 2006, pp. 123-136.

[103] Marvin L. Minsky. Computation: finite and infinite machines. PrenticeHall, Inc., 1967.

[104] Leonardo Mendonça de Moura and Nikolaj Bjørner. "Z3: An Efficient SMT Solver." In: Proceedings of the 14th International Conference on Tools and Algorithms for the Construction and Analysis of Systems, TACAS 2008, Budapest, Hungary, March 2008. Vol. 4963. Lecture Notes in Computer Science. Springer, 2008, pp. 337-340.

[105] Leonor Prensa Nieto. "Completeness of the Owicki-Gries System for Parameterized Parallel Programs." In: Proceedings of the 15th International Parallel \&5 Distributed Processing Symposium, IPDPS-01, San Francisco, CA, USA, April 2001. IEEE Computer Society, 2001, p. 150.

[106] Susan S. Owicki and David Gries. "An Axiomatic Proof Technique for Parallel Programs I.” In: Acta Informatica 6 (1976), pp. 319-340.

[107] Oded Padon, Giuliano Losa, Mooly Sagiv, and Sharon Shoham. "Paxos made EPR: decidable reasoning about distributed protocols." In: Proc. ACM Program. Lang. 1.OOPSLA (2017), 108:1-108:31.

[108] Paritosh K. Pandya and Mathai Joseph. "P - A Logic - A Compositional Proof System for Distributed Programs." In: Distributed Comput. 5 (1991), pp. 37-54. 
[109] Amir Pnueli, Sitvanit Ruah, and Lenore D. Zuck. "Automatic Deductive Verification with Invisible Invariants." In: Proceedings of the 7th International Conference on Tools and Algorithms for the Construction and Analysis of Systems, TACAS 2001, Genova, Italy, April 2001. Vol. 2031. Lecture Notes in Computer Science. Springer, 2001, pp. 8297.

[110] Amir Pnueli, Jessie Xu, and Lenore D. Zuck. "Liveness with (0, 1, infty)-Counter Abstraction." In: Proceedings of the 14th International Conference on Computer Aided Verification, CAV 2002, Copenhagen, Denmark, July 2002. Vol. 2404. Lecture Notes in Computer Science. Springer, 2002, pp. 107-122.

[111] Jean-Pierre Queille and Joseph Sifakis. "Specification and verification of concurrent systems in CESAR." In: Proceedings of the 5th International Symposium on Programming, Torino, Italy, April 1982. Vol. 137. Springer, 1982, pp. 337-351.

[112] W. Reisig. Petri nets: an introduction. Vol. 4. Springer Science \& Business Media, 2012.

[113] Peter Z. Revesz. "A closed-form evaluation for Datalog queries with integer (gap)-order constraints." In: Theoretical Computer Science 116.1 (1993), pp. 117-149.

[114] Jun Shirako, David M. Peixotto, Vivek Sarkar, and William N. Scherer III. "Phaser accumulators: A new reduction construct for dynamic parallelism." In: Proceedings of the 23rd IEEE International Symposium on Parallel and Distributed Processing, IPDPS 2009, Rome, Italy, May 2009. IEEE, 2009, pp. 1-12.

[115] Jun Shirako, David M. Peixotto, Vivek Sarkar, and William N. Scherer III. "Phasers: a unified deadlock-free construct for collective and pointto-point synchronization." In: Proceedings of the 22nd Annual International Conference on Supercomputing, ICS 2008, Island of Kos, Greece, June 2008. ACM, 2008, pp. 277-288.

[116] Abraham Silberschatz, Peter Baer Galvin, and Greg Gagne. Operating systems concepts with Java, 6th Edition. Wiley, 2004.

[117] A. Prasad Sistla. "Safety, Liveness and Fairness in Temporal Logic." In: Formal Aspects Comput. 6.5 (1994), pp. 495-512.

[118] Jean Souyris, Erwan Le Pavec, Guillaume Himbert, Guillaume Borios, Victor Jégu, and Reinhold Heckmann. "Computing the worst case execution time of an avionics program by abstract interpretation." In: 5th International Workshop on Worst-Case Execution Time Analysis, WCET'05. Vol. 1. Schloss Dagstuhl-Leibniz-Zentrum fuer Informatik, 2007. 
[119] T. K. Srikanth and Sam Toueg. "Simulating Authenticated Broadcasts to Derive Simple Fault-Tolerant Algorithms." In: Distributed Comput. 2.2 (1987), pp. 80-94.

[120] Maarten van Steen and Andrew S. Tanenbaum. "A brief introduction to distributed systems." In: Computing 98.10 (2016), pp. 967-1009.

[121] Ilina Stoilkovska, Igor V. Konnov, Josef Widder, and Florian Zuleger. "Verifying Safety of Synchronous Fault-Tolerant Algorithms by Bounded Model Checking." In: Proceedings of the 25th International Conference on Tools and Algorithms for the Construction and Analysis of Systems, TACAS 2019, Prague, Czech Republic, April 2019. Vol. 11428. Lecture Notes in Computer Science. Springer, 2019, pp. $357-374$.

[122] Carl A. Sunshine. "A brief history of computer networking." In: Computer Network Architectures and Protocols. Springer, 1989, pp. 3-6.

[123] Andrew S. Tanenbaum and Maarten van Steen. Distributed systems principles and paradigms, 2nd Edition. Pearson Education, 2007.

[124] Salvatore La Torre, P. Madhusudan, and Gennaro Parlato. "ModelChecking Parameterized Concurrent Programs Using Linear Interfaces." In: Proceedings of the 22nd International Conference on Computer Aided Verification, CAV 2010, Edinburgh, UK, July 2010. Vol. 6174. Lecture Notes in Computer Science. Springer, 2010, pp. 629 644 .

[125] Alan Turing. "Checking a large routine." In: Report of a Conference on High Speed Automatic Calculating Machines. 1949, pp. 67-69.

[126] Leslie G. Valiant. "A Bridging Model for Parallel Computation." In: Commun. ACM 33.8 (1990), pp. 103-111.

[127] Georg Weissenbacher, Daniel Kroening, and Sharad Malik. "Wolverine: Battling Bugs with Interpolants - (Competition Contribution)." In: Proceedings of the 18th International Conference on Tools and Algorithms for the Construction and Analysis of Systems, TACAS 2012, Tallinn, Estonia, March 2012. Vol. 7214. Lecture Notes in Computer Science. Springer, 2012, pp. 556-558.

[128] Marc Willebeek-LeMair and Anthony P. Reeves. "Strategies for dynamic load balancing on highly parallel computers." In: IEEE Transactions on parallel and distributed systems 4.9 (1993), pp. 979-993.

[129] Michael Zhivich and Robert K. Cunningham. "The Real Cost of Software Errors." In: IEEE Secur. Priv. 7.2 (2009), pp. 87-90.

[130] Lenore D. Zuck and Amir Pnueli. "Model checking and abstraction to the aid of parameterized systems (a survey)." In: Comput. Lang. Syst. Struct. 30.3-4 (2004), pp. 139-169. 
Department of Computer and Information Science

Linköpings universitet

\section{Dissertations}

\section{Linköping Studies in Science and Technology \\ Linköping Studies in Arts and Science \\ Linköping Studies in Statistics \\ Linköping Studies in Information Science}

Linköping Studies in Science and Technology

No 14 Anders Haraldsson: A Program Manipulation System Based on Partial Evaluation, 1977, ISBN $91-$ 7372-144-1.

No 17 Bengt Magnhagen: Probability Based Verification of Time Margins in Digital Designs, 1977, ISBN 91-7372157-3.

No 18 Mats Cedwall: Semantisk analys av processbeskrivningar i naturligt språk, 1977, ISBN 91- 7372168-9.

No 22 Jaak Urmi: A Machine Independent LISP Compiler and its Implications for Ideal Hardware, 1978, ISBN 91-7372-188-3.

No 33 Tore Risch: Compilation of Multiple File Queries in a Meta-Database System, 1978, ISBN 91- 7372-232-4.

No 51 Erland Jungert: Synthesizing Database Structures from a User Oriented Data Model, 1980, ISBN 917372-387-8.

No 54 Sture Hägglund: Contributions to the Development of Methods and Tools for Interactive Design of Applications Software, 1980, ISBN 91-7372-404-1.

No 55 Pär Emanuelson: Performance Enhancement in a Well-Structured Pattern Matcher through Partial Evaluation, 1980, ISBN 91-7372-403-3.

No 58 Bengt Johnsson, Bertil Andersson: The HumanComputer Interface in Commercial Systems, 1981, ISBN 91-7372-414-9.

No 69 H. Jan Komorowski: A Specification of an Abstract Prolog Machine and its Application to Partial Evaluation, 1981, ISBN 91-7372-479-3.

No 71 René Reboh: Knowledge Engineering Techniques and Tools for Expert Systems, 1981, ISBN 91-7372489-0.

No 77 Östen Oskarsson: Mechanisms of Modifiability in large Software Systems, 1982, ISBN 91- 7372-527-7.

No 94 Hans Lunell: Code Generator Writing Systems, 1983, ISBN 91-7372-652-4.

No 97 Andrzej Lingas: Advances in Minimum Weight Triangulation, 1983, ISBN 91-7372-660-5.

No 109 Peter Fritzson: Towards a Distributed Programming Environment based on Incremental Compilation, 1984, ISBN 91-7372-801-2.

No 111 Erik Tengvald: The Design of Expert Planning Systems. An Experimental Operations Planning System for Turning, 1984, ISBN 91-7372- 805-5.

No 155 Christos Levcopoulos: Heuristics for Minimum Decompositions of Polygons, 1987, ISBN 91-7870133-3.

No 165 James W. Goodwin: A Theory and System for NonMonotonic Reasoning, 1987, ISBN 91-7870-183-X.

No 170 Zebo Peng: A Formal Methodology for Automated Synthesis of VLSI Systems, 1987, ISBN 91-7870-225-9.

No 174 Johan Fagerström: A Paradigm and System for Design of Distributed Systems, 1988, ISBN 91-7870301-8.
No 192 Dimiter Driankov: Towards a Many Valued Logic of Quantified Belief, 1988, ISBN 91-7870-374-3.

No 213 Lin Padgham: Non-Monotonic Inheritance for an Object Oriented Knowledge Base, 1989, ISBN 917870-485-5.

No 214 Tony Larsson: A Formal Hardware Description and Verification Method, 1989, ISBN 91-7870-517-7.

No 221 Michael Reinfrank: Fundamentals and Logical Foundations of Truth Maintenance, 1989, ISBN 917870-546-0.

No 239 Jonas Löwgren: Knowledge-Based Design Support and Discourse Management in User Interface Management Systems, 1991, ISBN 91-7870-720-X.

No 244 Henrik Eriksson: Meta-Tool Support for Knowledge Acquisition, 1991, ISBN 91-7870-746-3.

No 252 Peter Eklund: An Epistemic Approach to Interactive Design in Multiple Inheritance Hierarchies, 1991, ISBN 91-7870-784-6.

No 258 Patrick Doherty: NML3 - A Non-Monotonic Formalism with Explicit Defaults, 1991, ISBN 917870-816-8.

No 260 Nahid Shahmehri: Generalized Algorithmic Debugging, 1991, ISBN 91-7870-828-1.

No 264 Nils Dahlbäck: Representation of DiscourseCognitive and Computational Aspects, 1992, ISBN 91-7870-850-8.

No 265 Ulf Nilsson: Abstract Interpretations and Abstract Machines: Contributions to a Methodology for the Implementation of Logic Programs, 1992, ISBN 917870-858-3.

No 270 Ralph Rönnquist: Theory and Practice of Tensebound Object References, 1992, ISBN 91-7870-873-7.

No 273 Björn Fjellborg: Pipeline Extraction for VLSI Data Path Synthesis, 1992, ISBN 91-7870-880-X.

No 276 Staffan Bonnier: A Formal Basis for Horn Clause Logic with External Polymorphic Functions, 1992, ISBN 91-7870-896-6.

No 277 Kristian Sandahl: Developing Knowledge Management Systems with an Active Expert Methodology, 1992, ISBN 91-7870-897-4.

No 281 Christer Bäckström: Computational Complexity of Reasoning about Plans, 1992, ISBN 91-7870-979-2.

No 292 Mats Wirén: Studies in Incremental Natural Language Analysis, 1992, ISBN 91-7871-027-8.

No 297 Mariam Kamkar: Interprocedural Dynamic Slicing with Applications to Debugging and Testing, 1993, ISBN 91-7871-065-0.

No 302 Tingting Zhang: A Study in Diagnosis Using Classification and Defaults, 1993, ISBN 91-7871-0782.

No 312 Arne Jönsson: Dialogue Management for Natural Language Interfaces - An Empirical Approach, 1993, ISBN 91-7871-110-X.

No 338 Simin Nadjm-Tehrani: Reactive Systems in Physical Environments: Compositional Modelling and Framework for Verification, 1994, ISBN 91-7871-237-8. 
No 371 Bengt Savén: Business Models for Decision Support and Learning. A Study of Discrete-Event Manufacturing Simulation at Asea/ABB 1968-1993, 1995, ISBN 91-7871-494-X.

No 375 Ulf Söderman: Conceptual Modelling of Mode Switching Physical Systems, 1995, ISBN 91-7871-5164.

No 383 Andreas Kågedal: Exploiting Groundness in Logic Programs, 1995, ISBN 91-7871-538-5.

No 396 George Fodor: Ontological Control, Description, Identification and Recovery from Problematic Control Situations, 1995, ISBN 91-7871-603-9.

No 413 Mikael Pettersson: Compiling Natural Semantics, 1995, ISBN 91-7871-641-1.

No 414 Xinli Gu: RT Level Testability Improvement by Testability Analysis and Transformations, 1996, ISBN 91-7871-654-3.

No 416 Hua Shu: Distributed Default Reasoning, 1996, ISBN 91-7871-665-9.

No 429 Jaime Villegas: Simulation Supported Industrial Training from an Organisational Learning Perspective - Development and Evaluation of the SSIT Method, 1996, ISBN 91-7871-700-0.

No 431 Peter Jonsson: Studies in Action Planning: Algorithms and Complexity, 1996, ISBN 91-7871-7043.

No 437 Johan Boye: Directional Types in Logic Programming, 1996, ISBN 91-7871-725-6.

No 439 Cecilia Sjöberg: Activities, Voices and Arenas: Participatory Design in Practice, 1996, ISBN 91-7871728-0.

No 448 Patrick Lambrix: Part-Whole Reasoning in Description Logics, 1996, ISBN 91-7871-820-1.

No 452 Kjell Orsborn: On Extensible and Object-Relational Database Technology for Finite Element Analysis Applications, 1996, ISBN 91-7871-827-9.

No 459 Olof Johansson: Development Environments for Complex Product Models, 1996, ISBN 91-7871-855-4.

No 461 Lena Strömbäck: User-Defined Constructions in Unification-Based Formalisms, 1997, ISBN 91-7871857-0.

No 462 Lars Degerstedt: Tabulation-based Logic Programming: A Multi-Level View of Query Answering, 1996, ISBN 91-7871-858-9.

No 475 Fredrik Nilsson: Strategi och ekonomisk styrning En studie av hur ekonomiska styrsystem utformas och används efter företagsförvärv, 1997, ISBN 917871-914-3.

No 480 Mikael Lindvall: An Empirical Study of Requirements-Driven Impact Analysis in Object-Oriented Software Evolution, 1997, ISBN 91-7871-927-5.

No 485 Göran Forslund: Opinion-Based Systems: The Cooperative Perspective on Knowledge-Based Decision Support, 1997, ISBN 91-7871-938-0.

No 494 Martin Sköld: Active Database Management Systems for Monitoring and Control, 1997, ISBN 917219-002-7.

No 495 Hans Olsén: Automatic Verification of Petri Nets in a CLP framework, 1997, ISBN 91-7219-011-6.

No 498 Thomas Drakengren: Algorithms and Complexity for Temporal and Spatial Formalisms, 1997, ISBN 917219-019-1.

No 502 Jakob Axelsson: Analysis and Synthesis of Heterogeneous Real-Time Systems, 1997, ISBN 91-7219-035-3.
No 503 Johan Ringström: Compiler Generation for DataParallel Programming Languages from Two-Level Semantics Specifications, 1997, ISBN 91-7219-045-0.

No 512 Anna Moberg: Närhet och distans - Studier av kommunikationsmönster i satellitkontor och flexibla kontor, 1997, ISBN 91-7219-119-8.

No 520 Mikael Ronström: Design and Modelling of a Parallel Data Server for Telecom Applications, 1998, ISBN 91-7219-169-4.

No 522 Niclas Ohlsson: Towards Effective Fault Prevention - An Empirical Study in Software Engineering, 1998, ISBN 91-7219-176-7.

No 526 Joachim Karlsson: A Systematic Approach for Prioritizing Software Requirements, 1998, ISBN 917219-184-8.

No 530 Henrik Nilsson: Declarative Debugging for Lazy Functional Languages, 1998, ISBN 91-7219-197-X.

No 555 Jonas Hallberg: Timing Issues in High-Level Synthesis, 1998, ISBN 91-7219-369-7.

No 561 Ling Lin: Management of 1-D Sequence Data - From Discrete to Continuous, 1999, ISBN 91-7219-402-2.

No 563 Eva L Ragnemalm: Student Modelling based on Collaborative Dialogue with a Learning Companion, 1999, ISBN 91-7219-412-X.

No 567 Jörgen Lindström: Does Distance matter? On geographical dispersion in organisations, 1999, ISBN 917219-439-1.

No 582 Vanja Josifovski: Design, Implementation and Evaluation of a Distributed Mediator System for Data Integration, 1999, ISBN 91-7219-482-0.

No 589 Rita Kovordányi: Modeling and Simulating Inhibitory Mechanisms in Mental Image Reinterpretation - Towards Cooperative HumanComputer Creativity, 1999, ISBN 91-7219-506-1.

No 592 Mikael Ericsson: Supporting the Use of Design Knowledge - An Assessment of Commenting Agents, 1999, ISBN 91-7219-532-0.

No 593 Lars Karlsson: Actions, Interactions and Narratives, 1999, ISBN 91-7219-534-7.

No 594 C. G. Mikael Johansson: Social and Organizational Aspects of Requirements Engineering Methods - A practice-oriented approach, 1999, ISBN 91-7219-541$\mathrm{X}$.

No 595 Jörgen Hansson: Value-Driven Multi-Class Overload Management in Real-Time Database Systems, 1999, ISBN 91-7219-542-8.

No 596 Niklas Hallberg: Incorporating User Values in the Design of Information Systems and Services in the Public Sector: A Methods Approach, 1999, ISBN 917219-543-6.

No 597 Vivian Vimarlund: An Economic Perspective on the Analysis of Impacts of Information Technology: From Case Studies in Health-Care towards General Models and Theories, 1999, ISBN 91-7219-544-4.

No 598 Johan Jenvald: Methods and Tools in ComputerSupported Taskforce Training, 1999, ISBN 91-7219547-9.

No 607 Magnus Merkel: Understanding and enhancing translation by parallel text processing, 1999, ISBN 917219-614-9.

No 611 Silvia Coradeschi: Anchoring symbols to sensory data, 1999, ISBN 91-7219-623-8.

No 613 Man Lin: Analysis and Synthesis of Reactive Systems: A Generic Layered Architecture Perspective, 1999, ISBN 91-7219-630-0. 
No 618 Jimmy Tjäder: Systemimplementering i praktiken En studie av logiker i fyra projekt, 1999, ISBN 917219-657-2.

No 627 Vadim Engelson: Tools for Design, Interactive Simulation, and Visualization of Object-Oriented Models in Scientific Computing, 2000, ISBN 91-7219709-9.

No 637 Esa Falkenroth: Database Technology for Control and Simulation, 2000, ISBN 91-7219-766-8.

No 639 Per-Arne Persson: Bringing Power and Knowledge Together: Information Systems Design for Autonomy and Control in Command Work, 2000, ISBN 91-7219796-X.

No 660 Erik Larsson: An Integrated System-Level Design for Testability Methodology, 2000, ISBN 91-7219-890-7.

No 688 Marcus Bjäreland: Model-based Execution Monitoring, 2001, ISBN 91-7373-016-5.

No 689 Joakim Gustafsson: Extending Temporal Action Logic, 2001, ISBN 91-7373-017-3.

No 720 Carl-Johan Petri: Organizational Information Provision - Managing Mandatory and Discretionary Use of Information Technology, 2001, ISBN 91-7373-1269.

No 724 Paul Scerri: Designing Agents for Systems with Adjustable Autonomy, 2001, ISBN 91-7373-207-9.

No 725 Tim Heyer: Semantic Inspection of Software Artifacts: From Theory to Practice, 2001, ISBN 917373-208-7.

No 726 Pär Carlshamre: A Usability Perspective on Requirements Engineering - From Methodology to Product Development, 2001, ISBN 91-7373-212-5.

No 732 Juha Takkinen: From Information Management to Task Management in Electronic Mail, 2002, ISBN 917373-258-3.

No 745 Johan Åberg: Live Help Systems: An Approach to Intelligent Help for Web Information Systems, 2002, ISBN 91-7373-311-3.

No 746 Rego Granlund: Monitoring Distributed Teamwork Training, 2002, ISBN 91-7373-312-1.

No 757 Henrik André-Jönsson: Indexing Strategies for Time Series Data, 2002, ISBN 917373-346-6.

No 747 Anneli Hagdahl: Development of IT-supported Interorganisational Collaboration - A Case Study in the Swedish Public Sector, 2002, ISBN 91-7373-314-8.

No 749 Sofie Pilemalm: Information Technology for NonProfit Organisations - Extended Participatory Design of an Information System for Trade Union Shop Stewards, 2002, ISBN 91-7373-318-0.

No 765 Stefan Holmlid: Adapting users: Towards a theory of use quality, 2002, ISBN 91-7373-397-0.

No 771 Magnus Morin: Multimedia Representations of Distributed Tactical Operations, 2002, ISBN 91-7373-4217.

No 772 Pawel Pietrzak: A Type-Based Framework for Locating Errors in Constraint Logic Programs, 2002, ISBN 91-7373-422-5.

No 758 Erik Berglund: Library Communication Among Programmers Worldwide, 2002, ISBN 91-7373-349-0.

No 774 Choong-ho Yi: Modelling Object-Oriented Dynamic Systems Using a Logic-Based Framework, 2002, ISBN 91-7373-424-1.

No 779 Mathias Broxvall: A Study in the Computational Complexity of Temporal Reasoning, 2002, ISBN 917373-440-3.
No 793 Asmus Pandikow: A Generic Principle for Enabling Interoperability of Structured and Object-Oriented Analysis and Design Tools, 2002, ISBN 91-7373-479-9.

No 785 Lars Hult: Publika Informationstjänster. En studie av den Internetbaserade encyklopedins bruksegenskaper, 2003, ISBN 91-7373-461-6.

No 800 Lars Taxén: A Framework for the Coordination of Complex Systems' Development, 2003, ISBN 917373-604-X.

No 808 Klas Gäre: Tre perspektiv på förväntningar och förändringar $i$ samband med införande av informationssystem, 2003, ISBN 91-7373-618-X.

No 821 Mikael Kindborg: Concurrent Comics programming of social agents by children, 2003, ISBN 91-7373-651-1.

No 823 Christina Ölvingson: On Development of Information Systems with GIS Functionality in Public Health Informatics: A Requirements Engineering Approach, 2003, ISBN 91-7373-656-2.

No 828 Tobias Ritzau: Memory Efficient Hard Real-Time Garbage Collection, 2003, ISBN 91-7373-666-X.

No 833 Paul Pop: Analysis and Synthesis of Communication-Intensive Heterogeneous Real-Time Systems, 2003, ISBN 91-7373-683-X.

No 852 Johan Moe: Observing the Dynamic Behaviour of Large Distributed Systems to Improve Development and Testing - An Empirical Study in Software Engineering, 2003, ISBN 91-7373-779-8.

No 867 Erik Herzog: An Approach to Systems Engineering Tool Data Representation and Exchange, 2004, ISBN 91-7373-929-4.

No 872 Aseel Berglund: Augmenting the Remote Control: Studies in Complex Information Navigation for Digital TV, 2004, ISBN 91-7373-940-5.

No 869 Jo Skåmedal: Telecommuting's Implications on Travel and Travel Patterns, 2004, ISBN 91-7373-935-9.

No 870 Linda Askenäs: The Roles of IT - Studies of Organising when Implementing and Using Enterprise Systems, 2004, ISBN 91-7373-936-7.

No 874 Annika Flycht-Eriksson: Design and Use of Ontologies in Information-Providing Dialogue Systems, 2004, ISBN 91-7373-947-2.

No 873 Peter Bunus: Debugging Techniques for EquationBased Languages, 2004, ISBN 91-7373-941-3.

No 876 Jonas Mellin: Resource-Predictable and Efficient Monitoring of Events, 2004, ISBN 91-7373-956-1.

No 883 Magnus Bång: Computing at the Speed of Paper: Ubiquitous Computing Environments for Healthcare Professionals, 2004, ISBN 91-7373-971-5.

No 882 Robert Eklund: Disfluency in Swedish humanhuman and human-machine travel booking dialogues, 2004, ISBN 91-7373-966-9.

No 887 Anders Lindström: English and other Foreign Linguistic Elements in Spoken Swedish. Studies of Productive Processes and their Modelling using Finite-State Tools, 2004, ISBN 91-7373-981-2.

No 889 Zhiping Wang: Capacity-Constrained Production-inventory systems - Modelling and Analysis in both a traditional and an e-business context, 2004, ISBN 9185295-08-6

No 893 Pernilla Qvarfordt: Eyes on Multimodal Interaction, 2004, ISBN 91-85295-30-2.

No 910 Magnus Kald: In the Borderland between Strategy and Management Control - Theoretical Framework and Empirical Evidence, 2004, ISBN 91-85295-82-5. 
No 918 Jonas Lundberg: Shaping Electronic News: Genre Perspectives on Interaction Design, 2004, ISBN 9185297-14-3.

No 900 Mattias Arvola: Shades of use: The dynamics of interaction design for sociable use, 2004, ISBN 9185295-42-6.

No 920 Luis Alejandro Cortés: Verification and Scheduling Techniques for Real-Time Embedded Systems, 2004, ISBN 91-85297-21-6.

No 929 Diana Szentivanyi: Performance Studies of FaultTolerant Middleware, 2005, ISBN 91-85297-58-5.

No 933 Mikael Cäker: Management Accounting as Constructing and Opposing Customer Focus: Three Case Studies on Management Accounting and Customer Relations, 2005, ISBN 91-85297-64-X.

No 937 Jonas Kvarnström: TALplanner and Other Extensions to Temporal Action Logic, 2005, ISBN 9185297-75-5.

No 938 Bourhane Kadmiry: Fuzzy Gain-Scheduled Visual Servoing for Unmanned Helicopter, 2005, ISBN 9185297-76-3.

No 945 Gert Jervan: Hybrid Built-In Self-Test and Test Generation Techniques for Digital Systems, 2005, ISBN 91-85297-97-6.

No 946 Anders Arpteg: Intelligent Semi-Structured Information Extraction, 2005, ISBN 91-85297-98-4.

No 947 Ola Angelsmark: Constructing Algorithms for Constraint Satisfaction and Related Problems - Methods and Applications, 2005, ISBN 91-85297-99-2.

No 963 Calin Curescu: Utility-based Optimisation of Resource Allocation for Wireless Networks, 2005, ISBN 91-85457-07-8.

No 972 Björn Johansson: Joint Control in Dynamic Situations, 2005, ISBN 91-85457-31-0.

No 974 Dan Lawesson: An Approach to Diagnosability Analysis for Interacting Finite State Systems, 2005, ISBN 91-85457-39-6.

No 979 Claudiu Duma: Security and Trust Mechanisms for Groups in Distributed Services, 2005, ISBN 91-8545754-X.

No 983 Sorin Manolache: Analysis and Optimisation of Real-Time Systems with Stochastic Behaviour, 2005, ISBN 91-85457-60-4.

No 986 Yuxiao Zhao: Standards-Based Application Integration for Business-to-Business Communications, 2005, ISBN 91-85457-66-3.

No 1004 Patrik Haslum: Admissible Heuristics for Automated Planning, 2006, ISBN 91-85497-28-2.

No 1005 Aleksandra Tešanovic: Developing Reusable and Reconfigurable Real-Time Software using Aspects and Components, 2006, ISBN 91-85497-29-0.

No 1008 David Dinka: Role, Identity and Work: Extending the design and development agenda, 2006, ISBN 9185497-42-8.

No 1009 Iakov Nakhimovski: Contributions to the Modeling and Simulation of Mechanical Systems with Detailed Contact Analysis, 2006, ISBN 91-85497-43-X.

No 1013 Wilhelm Dahllöf: Exact Algorithms for Exact Satisfiability Problems, 2006, ISBN 91-85523-97-6.

No 1016 Levon Saldamli: PDEModelica - A High-Level Language for Modeling with Partial Differential Equations, 2006, ISBN 91-85523-84-4.

No 1017 Daniel Karlsson: Verification of Component-based Embedded System Designs, 2006, ISBN 91-85523-79-8
No 1018 Ioan Chisalita: Communication and Networking Techniques for Traffic Safety Systems, 2006, ISBN 9185523-77-1.

No 1019 Tarja Susi: The Puzzle of Social Activity - The Significance of Tools in Cognition and Cooperation, 2006, ISBN 91-85523-71-2.

No 1021 Andrzej Bednarski: Integrated Optimal Code Generation for Digital Signal Processors, 2006, ISBN 9185523-69-0.

No 1022 Peter Aronsson: Automatic Parallelization of Equation-Based Simulation Programs, 2006, ISBN 9185523-68-2.

No 1030 Robert Nilsson: A Mutation-based Framework for Automated Testing of Timeliness, 2006, ISBN 9185523-35-6.

No 1034 Jon Edvardsson: Techniques for Automatic Generation of Tests from Programs and Specifications, 2006, ISBN 91-85523-31-3.

No 1035 Vaida Jakoniene: Integration of Biological Data, 2006, ISBN 91-85523-28-3.

No 1045 Genevieve Gorrell: Generalized Hebbian Algorithms for Dimensionality Reduction in Natural Language Processing, 2006, ISBN 91-85643-88-2.

No 1051 Yu-Hsing Huang: Having a New Pair of Glasses Applying Systemic Accident Models on Road Safety, 2006, ISBN 91-85643-64-5.

No 1054 Åsa Hedenskog: Perceive those things which cannot be seen - A Cognitive Systems Engineering perspective on requirements management, 2006, ISBN 91-85643-57-2.

No 1061 Cécile Åberg: An Evaluation Platform for Semantic Web Technology, 2007, ISBN 91-85643-31-9.

No 1073 Mats Grindal: Handling Combinatorial Explosion in Software Testing, 2007, ISBN 978-91-85715-74-9.

No 1075 Almut Herzog: Usable Security Policies for Runtime Environments, 2007, ISBN 978-91-85715-65-7.

No 1079 Magnus Wahlström: Algorithms, measures, and upper bounds for Satisfiability and related problems, 2007, ISBN 978-91-85715-55-8.

No 1083 Jesper Andersson: Dynamic Software Architectures, 2007, ISBN 978-91-85715-46-6.

No 1086 Ulf Johansson: Obtaining Accurate and Comprehensible Data Mining Models - An Evolutionary Approach, 2007, ISBN 978-91-85715-34-3.

No 1089 Traian Pop: Analysis and Optimisation of Distributed Embedded Systems with Heterogeneous Scheduling Policies, 2007, ISBN 978-91-85715-27-5.

No 1091 Gustav Nordh: Complexity Dichotomies for CSPrelated Problems, 2007, ISBN 978-91-85715-20-6.

No 1106 Per Ola Kristensson: Discrete and Continuous Shape Writing for Text Entry and Control, 2007, ISBN 97891-85831-77-7.

No 1110 He Tan: Aligning Biomedical Ontologies, 2007, ISBN 978-91-85831-56-2.

No 1112 Jessica Lindblom: Minding the body - Interacting socially through embodied action, 2007, ISBN 978-9185831-48-7.

No 1113 Pontus Wärnestål: Dialogue Behavior Management in Conversational Recommender Systems, 2007, ISBN 978-91-85831-47-0.

No 1120 Thomas Gustafsson: Management of Real-Time Data Consistency and Transient Overloads in Embedded Systems, 2007, ISBN 978-91-85831-33-3. 
No 1127 Alexandru Andrei: Energy Efficient and Predictable Design of Real-time Embedded Systems, 2007, ISBN 978-91-85831-06-7.

No 1139 Per Wikberg: Eliciting Knowledge from Experts in Modeling of Complex Systems: Managing Variation and Interactions, 2007, ISBN 978-91-85895-66-3.

No 1143 Mehdi Amirijoo: QoS Control of Real-Time Data Services under Uncertain Workload, 2007, ISBN 97891-85895-49-6.

No 1150 Sanny Syberfeldt: Optimistic Replication with Forward Conflict Resolution in Distributed Real-Time Databases, 2007, ISBN 978-91-85895-27-4.

No 1155 Beatrice Alenljung: Envisioning a Future Decision Support System for Requirements Engineering - A Holistic and Human-centred Perspective, 2008, ISBN 978-91-85895-11-3.

No 1156 Artur Wilk: Types for XML with Application to Xcerpt, 2008, ISBN 978-91-85895-08-3.

No 1183 Adrian Pop: Integrated Model-Driven Development Environments for Equation-Based Object-Oriented Languages, 2008, ISBN 978-91-7393-895-2.

No 1185 Jörgen Skågeby: Gifting Technologies Ethnographic Studies of End-users and Social Media Sharing, 2008, ISBN 978-91-7393-892-1.

No 1187 Imad-Eldin Ali Abugessaisa: Analytical tools and information-sharing methods supporting road safety organizations, 2008, ISBN 978-91-7393-887-7.

No 1204 H. Joe Steinhauer: A Representation Scheme for Description and Reconstruction of Object Configurations Based on Qualitative Relations, 2008, ISBN 978-91-7393-823-5.

No 1222 Anders Larsson: Test Optimization for Core-based System-on-Chip, 2008, ISBN 978-91-7393-768-9.

No 1238 Andreas Borg: Processes and Models for Capacity Requirements in Telecommunication Systems, 2009, ISBN 978-91-7393-700-9.

No 1240 Fredrik Heintz: DyKnow: A Stream-Based Knowledge Processing Middleware Framework, 2009, ISBN 978-91-7393-696-5.

No 1241 Birgitta Lindström: Testability of Dynamic RealTime Systems, 2009, ISBN 978-91-7393-695-8.

No 1244 Eva Blomqvist: Semi-automatic Ontology Construction based on Patterns, 2009, ISBN 978-91-7393-683-5.

No 1249 Rogier Woltjer: Functional Modeling of Constraint Management in Aviation Safety and Command and Control, 2009, ISBN 978-91-7393-659-0.

No 1260 Gianpaolo Conte: Vision-Based Localization and Guidance for Unmanned Aerial Vehicles, 2009, ISBN 978-91-7393-603-3.

No 1262 AnnMarie Ericsson: Enabling Tool Support for Formal Analysis of ECA Rules, 2009, ISBN 978-91-7393598-2.

No 1266 Jiri Trnka: Exploring Tactical Command and Control: A Role-Playing Simulation Approach, 2009, ISBN 978-91-7393-571-5.

No 1268 Bahlol Rahimi: Supporting Collaborative Work through ICT - How End-users Think of and Adopt Integrated Health Information Systems, 2009, ISBN 978-91-7393-550-0.

No 1274 Fredrik Kuivinen: Algorithms and Hardness Results for Some Valued CSPs, 2009, ISBN 978-91-7393-525-8.

No 1281 Gunnar Mathiason: Virtual Full Replication for Scalable Distributed Real-Time Databases, 2009, ISBN 978-91-7393-503-6.
No 1290 Viacheslav Izosimov: Scheduling and Optimization of Fault-Tolerant Distributed Embedded Systems, 2009, ISBN 978-91-7393-482-4.

No 1294 Johan Thapper: Aspects of a Constraint Optimisation Problem, 2010, ISBN 978-91-7393-464-0.

No 1306 Susanna Nilsson: Augmentation in the Wild: User Centered Development and Evaluation of Augmented Reality Applications, 2010, ISBN 978-917393-416-9.

No 1313 Christer Thörn: On the Quality of Feature Models, 2010, ISBN 978-91-7393-394-0.

No 1321 Zhiyuan He: Temperature Aware and DefectProbability Driven Test Scheduling for System-onChip, 2010, ISBN 978-91-7393-378-0.

No 1333 David Broman: Meta-Languages and Semantics for Equation-Based Modeling and Simulation, 2010, ISBN 978-91-7393-335-3.

No 1337 Alexander Siemers: Contributions to Modelling and Visualisation of Multibody Systems Simulations with Detailed Contact Analysis, 2010, ISBN 978-91-7393317-9.

No 1354 Mikael Asplund: Disconnected Discoveries: Availability Studies in Partitioned Networks, 2010, ISBN 978-91-7393-278-3.

No 1359 Jana Rambusch: Mind Games Extended: Understanding Gameplay as Situated Activity, 2010, ISBN 978-91-7393-252-3.

No 1373 Sonia Sangari: Head Movement Correlates to Focus Assignment in Swedish, 2011, ISBN 978-91-7393-1540 .

No 1374 Jan-Erik Källhammer: Using False Alarms when Developing Automotive Active Safety Systems, 2011, ISBN 978-91-7393-153-3.

No 1375 Mattias Eriksson: Integrated Code Generation, 2011, ISBN 978-91-7393-147-2.

No 1381 Ola Leifler: Affordances and Constraints of Intelligent Decision Support for Military Command and Control - Three Case Studies of Support Systems, 2011, ISBN 978-91-7393-133-5.

No 1386 Soheil Samii: Quality-Driven Synthesis and Optimization of Embedded Control Systems, 2011, ISBN 978-91-7393-102-1.

No 1419 Erik Kuiper: Geographic Routing in Intermittentlyconnected Mobile Ad Hoc Networks: Algorithms and Performance Models, 2012, ISBN 978-91-7519981-8.

No 1451 Sara Stymne: Text Harmonization Strategies for Phrase-Based Statistical Machine Translation, 2012, ISBN 978-91-7519-887-3.

No 1455 Alberto Montebelli: Modeling the Role of Energy Management in Embodied Cognition, 2012, ISBN 978-91-7519-882-8.

No 1465 Mohammad Saifullah: Biologically-Based Interactive Neural Network Models for Visual Attention and Object Recognition, 2012, ISBN 978-91-7519-838-5.

No 1490 Tomas Bengtsson: Testing and Logic Optimization Techniques for Systems on Chip, 2012, ISBN 978-917519-742-5.

No 1481 David Byers: Improving Software Security by Preventing Known Vulnerabilities, 2012, ISBN 97891-7519-784-5.

No 1496 Tommy Färnqvist: Exploiting Structure in CSPrelated Problems, 2013, ISBN 978-91-7519-711-1. 
No 1503 John Wilander: Contributions to Specification, Implementation, and Execution of Secure Software, 2013, ISBN 978-91-7519-681-7.

No 1506 Magnus Ingmarsson: Creating and Enabling the Useful Service Discovery Experience, 2013, ISBN 97891-7519-662-6.

No 1547 Wladimir Schamai: Model-Based Verification of Dynamic System Behavior against Requirements: Method, Language, and Tool, 2013, ISBN 978-917519-505-6.

No 1551 Henrik Svensson: Simulations, 2013, ISBN 978-917519-491-2.

No 1559 Sergiu Rafiliu: Stability of Adaptive Distributed Real-Time Systems with Dynamic Resource Management, 2013, ISBN 978-91-7519-471-4.

No 1581 Usman Dastgeer: Performance-aware Component Composition for GPU-based Systems, 2014, ISBN 978-91-7519-383-0.

No 1602 Cai Li: Reinforcement Learning of Locomotion based on Central Pattern Generators, 2014, ISBN 978-917519-313-7.

No 1652 Roland Samlaus: An Integrated Development Environment with Enhanced Domain-Specific Interactive Model Validation, 2015, ISBN 978-917519-090-7.

No 1663 Hannes Uppman: On Some Combinatorial Optimization Problems: Algorithms and Complexity, 2015, ISBN 978-91-7519-072-3.

No 1664 Martin Sjölund: Tools and Methods for Analysis, Debugging, and Performance Improvement of Equation-Based Models, 2015, ISBN 978-91-7519-071-6.

No 1666 Kristian Stavåker: Contributions to Simulation of Modelica Models on Data-Parallel Multi-Core Architectures, 2015, ISBN 978-91-7519-068-6.

No 1680 Adrian Lifa: Hardware/Software Codesign of Embedded Systems with Reconfigurable and Heterogeneous Platforms, 2015, ISBN 978-91-7519-0402.

No 1685 Bogdan Tanasa: Timing Analysis of Distributed Embedded Systems with Stochastic Workload and Reliability Constraints, 2015, ISBN 978-91-7519-022-8.

No 1691 Håkan Warnquist: Troubleshooting Trucks Automated Planning and Diagnosis, 2015, ISBN 97891-7685-993-3.

No 1702 Nima Aghaee: Thermal Issues in Testing of Advanced Systems on Chip, 2015, ISBN 978-91-7685949-0.

No 1715 Maria Vasilevskaya: Security in Embedded Systems: A Model-Based Approach with Risk Metrics, 2015, ISBN 978-91-7685-917-9.

No 1729 Ke Jiang: Security-Driven Design of Real-Time Embedded System, 2016, ISBN 978-91-7685-884-4.

No 1733 Victor Lagerkvist: Strong Partial Clones and the Complexity of Constraint Satisfaction Problems: Limitations and Applications, 2016, ISBN 978-91-7685856-1.

No 1734 Chandan Roy: An Informed System Development Approach to Tropical Cyclone Track and Intensity Forecasting, 2016, ISBN 978-91-7685-854-7.

No 1746 Amir Aminifar: Analysis, Design, and Optimization of Embedded Control Systems, 2016, ISBN 978-917685-826-4.

No 1747 Ekhiotz Vergara: Energy Modelling and Fairness for Efficient Mobile Communication, 2016, ISBN 978-917685-822-6.
No 1748 Dag Sonntag: Chain Graphs - Interpretations, Expressiveness and Learning Algorithms, 2016, ISBN 978-91-7685-818-9.

No 1768 Anna Vapen: Web Authentication using ThirdParties in Untrusted Environments, 2016, ISBN 97891-7685-753-3.

No 1778 Magnus Jandinger: On a Need to Know Basis: A Conceptual and Methodological Framework for Modelling and Analysis of Information Demand in an Enterprise Context, 2016, ISBN 978-91-7685-713-7.

No 1798 Rahul Hiran: Collaborative Network Security: Targeting Wide-area Routing and Edge-network Attacks, 2016, ISBN 978-91-7685-662-8.

No 1813 Nicolas Melot: Algorithms and Framework for Energy Efficient Parallel Stream Computing on Many-Core Architectures, 2016, ISBN 978-91-7685623-9.

No 1823 Amy Rankin: Making Sense of Adaptations: Resilience in High-Risk Work, 2017, ISBN 978-917685-596-6.

No 1831 Lisa Malmberg: Building Design Capability in the Public Sector: Expanding the Horizons of Development, 2017, ISBN 978-91-7685-585-0.

No 1851 Marcus Bendtsen: Gated Bayesian Networks, 2017, ISBN 978-91-7685-525-6.

No 1852 Zlatan Dragisic: Completion of Ontologies and Ontology Networks, 2017, ISBN 978-91-7685-522-5.

No 1854 Meysam Aghighi: Computational Complexity of some Optimization Problems in Planning, 2017, ISBN 978-91-7685-519-5.

No 1863 Simon Ståhlberg: Methods for Detecting Unsolvable Planning Instances using Variable Projection, 2017, ISBN 978-91-7685-498-3.

No 1879 Karl Hammar: Content Ontology Design Patterns: Qualities, Methods, and Tools, 2017, ISBN 978-917685-454-9.

No 1887 Ivan Ukhov: System-Level Analysis and Design under Uncertainty, 2017, ISBN 978-91-7685-426-6.

No 1891 Valentina Ivanova: Fostering User Involvement in Ontology Alignment and Alignment Evaluation, 2017, ISBN 978-91-7685-403-7.

No 1902 Vengatanathan Krishnamoorthi: Efficient HTTPbased Adaptive Streaming of Linear and Interactive Videos, 2018, ISBN 978-91-7685-371-9.

No 1903 Lu Li: Programming Abstractions and Optimization Techniques for GPU-based Heterogeneous Systems, 2018, ISBN 978-91-7685-370-2.

No 1913 Jonas Rybing: Studying Simulations with Distributed Cognition, 2018, ISBN 978-91-7685-348-1.

No 1936 Leif Jonsson: Machine Learning-Based Bug Handling in Large-Scale Software Development, 2018, ISBN 978-91-7685-306-1.

No 1964 Arian Maghazeh: System-Level Design of GPUBased Embedded Systems, 2018, ISBN 978-91-7685175-3.

No 1967 Mahder Gebremedhin: Automatic and Explicit Parallelization Approaches for Equation Based Mathematical Modeling and Simulation, 2019, ISBN 978-91-7685-163-0.

No 1984 Anders Andersson: Distributed Moving Base Driving Simulators - Technology, Performance, and Requirements, 2019, ISBN 978-91-7685-090-9.

No 1993 Ulf Kargén: Scalable Dynamic Analysis of Binary Code, 2019, ISBN 978-91-7685-049-7. 
No 2001 Tim Overkamp: How Service Ideas Are Implemented: Ways of Framing and Addressing Service Transformation, 2019, ISBN 978-91-7685-025-1.

No 2006 Daniel de Leng: Robust Stream Reasoning Under Uncertainty, 2019, ISBN 978-91-7685-013-8.

No 2048 Biman Roy: Applications of Partial Polymorphisms in (Fine-Grained) Complexity of Constraint Satisfaction Problems, 2020, ISBN 978-91-7929-898-2.

No 2051 Olov Andersson: Learning to Make Safe Real-Time Decisions Under Uncertainty for Autonomous Robots, 2020, ISBN 978-91-7929-889-0.

No 2065 Vanessa Rodrigues: Designing for Resilience: Navigating Change in Service Systems, 2020, ISBN 978-91-7929-867-8.

No 2082 Robin Kurtz: Contributions to Semantic Dependency Parsing: Search, Learning, and Application, 2020, ISBN 978-91-7929-822-7.

No 2108 Shanai Ardi: Vulnerability and Risk Analysis Methods and Application in Large Scale Development of Secure Systems, 2021, ISBN 978-917929-744-2.

No 2125 Zeinab Ganjei: Parameterized Verification of Synchronized Concurrent Programs, 2021, ISBN 97891-7929-697-1.

Linköping Studies in Arts and Sciences

No504 Ing-Marie Jonsson: Social and Emotional Characteristics of Speech-based In-Vehicle Information Systems: Impact on Attitude and Driving Behaviour, 2009, ISBN 978-91-7393-478-7.

No 586 Fabian Segelström: Stakeholder Engagement for Service Design: How service designers identify and communicate insights, 2013, ISBN 978-91-7519-554-4.

No 618 Johan Blomkvist: Representing Future Situations of Service: Prototyping in Service Design, 2014, ISBN 978-91-7519-343-4.

No 620 Marcus Mast: Human-Robot Interaction for SemiAutonomous Assistive Robots, 2014, ISBN 978-917519-319-9.

No677 Peter Berggren: Assessing Shared Strategic Understanding, 2016, ISBN 978-91-7685-786-1.

No 695 Mattias Forsblad: Distributed cognition in home environments: The prospective memory and cognitive practices of older adults, 2016, ISBN 97891-7685-686-4.

No 787 Sara Nygårdhs: Adaptive behaviour in traffic: An individual road user perspective, 2020, ISBN 978-917929-857-9.

\section{Linköping Studies in Statistics}

No 9 Davood Shahsavani: Computer Experiments Designed to Explore and Approximate Complex Deterministic Models, 2008, ISBN 978-91-7393-976-8.

No 10 Karl Wahlin: Roadmap for Trend Detection and Assessment of Data Quality, 2008, ISBN 978-91-7393792-4.

No 11 Oleg Sysoev: Monotonic regression for large multivariate datasets, 2010, ISBN 978-91-7393-412-1.

No 13 Agné Burauskaite-Harju: Characterizing Temporal Change and Inter-Site Correlations in Daily and Subdaily Precipitation Extremes, 2011, ISBN 978-91-7393110-6.

No 14 Måns Magnusson: Scalable and Efficient Probabilistic Topic Model Inference for Textual Data, 2018, ISBN 978-91-7685-288-0.
No 15 Per Sidén: Scalable Bayesian spatial analysis with Gaussian Markov random fields, 2020, 978-91-7929818-0.

\section{Linköping Studies in Information Science}

No 1 Karin Axelsson: Metodisk systemstrukturering- att skapa samstämmighet mellan informationssystemarkitektur och verksamhet, 1998. ISBN 9172-19-296-8.

No 2 Stefan Cronholm: Metodverktyg och användbarhet en studie av datorstödd metodbaserad systemutveckling, 1998, ISBN 9172-19-299-2.

No 3 Anders Avdic: Användare och utvecklare - om anveckling med kalkylprogram, 1999. ISBN 91-7219606-8.

No 4 Owen Eriksson: Kommunikationskvalitet hos informationssystem och affärsprocesser, 2000, ISBN 917219-811-7.

No 5 Mikael Lind: Från system till process - kriterier för processbestämning vid verksamhetsanalys, 2001, ISBN 91-7373-067-X.

No 6 Ulf Melin: Koordination och informationssystem i företag och nätverk, 2002, ISBN 91-7373-278-8.

No 7 Pär J. Ågerfalk: Information Systems Actability - Understanding Information Technology as a Tool for Business Action and Communication, 2003, ISBN 917373-628-7.

No 8 Ulf Seigerroth: Att förstå och förändra systemutvecklingsverksamheter - en taxonomi för metautveckling, 2003, ISBN 91-7373-736-4.

No 9 Karin Hedström: Spår av datoriseringens värden Effekter av IT i äldreomsorg, 2004, ISBN 91-7373-9634.

No 10 Ewa Braf: Knowledge Demanded for Action Studies on Knowledge Mediation in Organisations, 2004, ISBN 91-85295-47-7.

No 11 Fredrik Karlsson: Method Configuration method and computerized tool support, 2005, ISBN 91-8529748-8.

No 12 Malin Nordström: Styrbar systemförvaltning - Att organisera systemförvaltningsverksamhet med hjälp av effektiva förvaltningsobjekt, 2005, ISBN 91-8529760-7.

No 13 Stefan Holgersson: Yrke: POLIS - Yrkeskunskap, motivation, IT-system och andra förutsättningar för polisarbete, 2005, ISBN 91-85299-43-X.

No 14 Benneth Christiansson, Marie-Therese Christiansson: Mötet mellan process och komponent - mot ett ramverk för en verksamhetsnära kravspecifikation vid anskaffning av komponentbaserade informationssystem, 2006, ISBN 91-85643$22-X$. 


\section{FACULTY OF SCIENCE AND ENGINEERING}

Linköping Studies in Science and Technology. Dissertations, No. 2125

Department of Computer and Information Science

Linköping University

SE-581 83 Linköping, Sweden

www.liu.se 